\title{
Three Essays on Identification and Estimation using Sample Combination in a Missing Data Context
}

by

\section{Marie-Hélène Felt}

A thesis submitted to the Faculty of Graduate and Postdoctoral Affairs in partial fulfillment of the requirements for the degree of

\section{Doctor of Philosophy \\ in}

\section{Economics}

\author{
Department of Economics \\ Carleton University \\ Ottawa, Ontario, Canada
}

(C) 2016

Marie-Hélène Felt 


\section{Abstract}

This thesis is concerned with identification and estimation in two different contexts of missing data. These data settings and their associated empirical challenges were faced during the analysis of micro survey data from the Bank of Canada on payment behaviors. However, the methodological approaches adopted or developed here are of wider interest and applicability. In both situations, identification is achieved by employing data combination strategies.

The first chapter uses panel data to understand the impact of retail payment innovations on cash usage while accounting for unobserved heterogeneity. The challenging feature of the data pertains to high rates (about 50 percent) of non-ignorable attrition. This missing data problem is addressed by the use of refreshment samples, which allow one to correct for potential attrition bias due to general forms of attrition instead of relying on rather restrictive assumptions. The methodological contribution is to provide identification of a three-period attrition probability function, and to discuss how to control simultaneously for non-ignorable attrition and item nonresponse.

The following chapters deal with the common missing data case in which the variables of interest to a research question are not all available in one single data set, and data combination is required. In contrast with most sample combination methods I propose, in the second chapter, an identification strategy that does not rely on either units or variables in common across the samples to be combined. Rather, I exploit the availability of a third sample where an aggregate distribution of the variables 
of interest, e.g. the distribution of their sum, is observed. Using deconvolution methods, I establish non-parametric identification of the joint distribution of interest by combining marginal distributions.

While the identification framework considered in Chapter 2 might seem quite specific, it is encountered in practice in multiple situations, and many potential applications exist. One of them occurs when individual and household surveys provide, for a given population, independent samples where the same variable is measured at the individual level in the one case and at the household level in the other. This is the data setting considered in the third chapter, which presents an empirical application of the identification procedure in the context of payment behavior analysis. In particular, in the absence of intra-household data, I investigate intra-household payment behaviors by combining individual-level and aggregated household-level payment survey data. 
To Martin. 


\section{Acknowledgements}

My first words of acknowledgements go to my thesis co-supervisors, Kim P. Huynh and Marcel Voia. There would be much more to say, but thank you for believing in me from the start and for guiding me along the way.

To my colleague and co-author Heng Chen, thanks for opening up the world of econometric identification to me. I've learned so much from working with you.

I would like to express my gratitude to Ben Fung, Assistant Director of Currency's Economic Research and Analysis division at the Bank of Canada, for welcoming me in the team and giving me the opportunity to access interesting data. My appreciation also goes to all my other colleagues, past and present, from the Bank.

To Lynda Khalaf, thanks for your support - in particular for spending hours helping me shape my SSHRC proposal - and, to put it briefly, thanks for caring. To David Gray from the University of Ottawa, thank you for carefully proofreading my manuscripts and for your insightful comments. Thank you to Sanjoy Sinha from Carleton University's School of Mathematics and Statistics and to Yuya Sasaki from Johns Hopkins University for examining my thesis; your critics and suggestions are greatly appreciated.

My work benefited substantially from conversations with visiting scholars at the Bank of Canada. I can't cite all of them, but I direct special thanks to Stéphane Bonhomme, Jean-Marie Dufour, Charlie Kahn, Victor Aguirregabiria, Thomas Lemieux and Arthur Lewbel for their specific feedbacks. I also acknowledge with gratitude 
useful correspondence with Carole Bernard, Aurore Delaigle and Peter Tankov concerning my particular identification problem.

I would like to extend my greetings to my fellow classmates. To Beatriz, Abeer and Charles, thanks for sharing advice and experiences. To my dear friend Chao Hui, thank you for your presence, open-mindedness and generosity. Thank you to the Department of Economics' administrative staff, in particular Marge Brooks and Renée Lortie, for supporting us and simplifying our lives.

Going back in time, I am grateful to Bruno Larue and Jean-Philippe Gervais from Université Laval for offering me my first research experience and inspiring me to do this $\mathrm{PhD}$.

Finally, I would like to express my love and gratitude to my husband Martin. Thank you for your patience and your understanding, and for being such a wonderful father to Jeanne, notre choupie. 


\section{Preface}

Chapter 1 is the product of collaboration with Heng Chen and Kim P. Huynh from the Bank of Canada's Currency Department. This work started in the winter term of 2013 in the context of a directed reading course in Econometric Methods for Policy Analysis. While getting to know the literature on program evaluation and treatment effects, I started exploring the Canadian Financial Monitor (CFM) data and its specific structure. Given the panel dimension and the presence of refreshment samples, the decision was taken to analyse the impact of payment innovations on cash usage by applying a semi-parametric moment-based approach proposed by Bhattacharya (2008), hereby controlling for both unobserved heterogeneity and non-ignorable attri-

tion. I performed all the data work, making the most of the Bank of Canada EDITH High Performance cluster and its parallel computing potential.

Preliminary results were presented during my first Ph.D. workshop in December 2013. Comments and suggestions received from my Ph.D. committee were then included in the paper - in particular, identification and estimation of a three-year attrition function. The Bank of Canada Staff Working Paper Retail Payment Innovations and Cash Usage: Accounting for Attrition Using Refreshment Samples was published in June 2014 (Chen et al., 2014). The paper further evolved based on journal reviewers' feedback with, most notably, consideration of item-non response. It was recently accepted for publication in the Journal of the Royal Statistical Society: Series A (Chen et al., forthcoming). 
In accordance with the permission from the Journal of the Royal Statistical Society: Series A, the version presented in this dissertation (including the tables, figures and Appendix A) is the final version that was seen and accepted for publication by the Editor. ${ }^{1}$ In addition, a Technical Appendix joined to the submission but not meant for publication, is reprinted in Appendix B.

Chapters 2 and 3 are original, unpublished, independent work by myself, MarieHélène Felt. No responsibility for the views expressed in this thesis should be attributed to the Bank of Canada.

\section{References}

Bhattacharya, D. (2008): "Inference in panel data models under attrition caused by unobservables," Journal of Econometrics, 144, 430-446.

Chen, H., M.-H. Felt, And K. P. Huynh (2014): "Retail payment innovations and cash usage: Accounting for attrition using refreshment samples," Staff Working Paper 2014-27, Bank of Canada.

_ (forthcoming): "Retail payment innovations and cash usage: Accounting for attrition using refreshment samples," Journal of the Royal Statistical Society: Series $A$.

\footnotetext{
${ }^{1}$ The word "paper" has been substituted with "chapter". Some typographical errors have been fixed.
} 


\section{Contents}

Abstract $\quad$ i

Acknowledgements $\quad$ iv

Preface vi vi

1 Retail Payment Innovations and Cash Usage: Accounting for Attrition Using Refreshment Samples 1

1.1 Introduction . . . . . . . . . . . . . . . . . . 1

1.2 Overview of Cash and Retail Payment Innovations . . . . . . . . 5

1.3 The Canadian Financial Monitor $\ldots \ldots \ldots \ldots$

1.3.1 Cash usage . . . . . . . . . . . . . . . . 7

1.3.2 Retail payment innovations . . . . . . . . . . . 8

1.3.3 Attrition and refreshment sample . . . . . . . . . . 9 9

1.4 Panel Data Estimation and Attrition . . . . . . . . . . . 10

1.5 Correcting for Non-ignorable Attrition . . . . . . . . . . . 12

1.5.1 Identification of attrition function without refreshment $\ldots .13$

1.5.2 Identification of attrition function with refreshment . . . . . 14

1.5.3 Identification with both attrition and item nonresponse . . . 16

1.6 Estimation and Results . . . . . . . . . . . . . . . . . . . . . . . . . 19

1.6.1 Estimation and Inference . . . . . . . . . . . . . . . . 19 
1.6.2 Impact of Retail Payment Innovations . . . . . . . . . . . . . 20

1.7 Conclusions ........................... 24

References ........................ 26

A Appendix to Chapter $1 \quad 42$

A.1 Variables Description . . . . . . . . . . . . . . . . 42

A.2 Attrition Functions and Moment Conditions . . . . . . . . . . . 44

A.3 Proof of Proposition $1 \ldots \ldots \ldots \ldots$

B Technical Appendix to Chapter 1 49

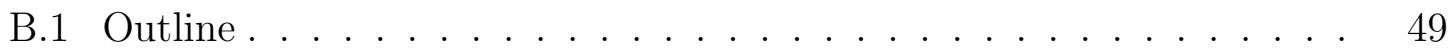

B.2 Correcting for Attrition in a Two-period panel with Refreshment . . . 50

B.2.1 Identification of a two-period attrition function with refreshment 50

B.2.2 Estimation via sieves . . . . . . . . . . . . . . 52

B.2.3 Asymptotic normality of SMD . . . . . . . . . . . 53

B.2.4 Asymptotic variance estimation . . . . . . . . . . 55

B.3 Practical Implementation . . . . . . . . . . . . . . . . . . 56

B.3.1 Sieve space and $g(\cdot)$ function . . . . . . . . . . . . 56

B.3.2 Optimization procedure .............. 57

B.3.3 Estimation algorithm . . . . . . . . . . . 57

B.4 Additional Results on Multiple Payment Innovations . . . . . . . . 58

B.5 Additional Results on Item Nonresponse . . . . . . . . . . . . . . 58

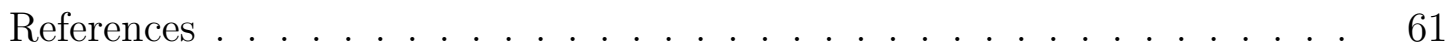

2 A Look Inside the Box: Combining Aggregate and Marginal Distri$\begin{array}{ll}\text { butions to Identify Joint Distributions } & 74\end{array}$

2.1 Introduction . . . . . . . . . . . . . . . . . 74

2.2 Identification Problem and Related Literatures . . . . . . . . . . . . 77

2.3 Model and Identification . . . . . . . . . . . . . . . . 81 
2.3.1 Model . . . . . . . . . . . . . . . . . . . . . . . . . . 81

2.3 .2 Main identification result . . . . . . . . . . . . . . . . 82

2.3 .3 Regularity conditions . . . . . . . . . . . . . 85

2.4 Restrictions and Extensions of the Model . . . . . . . . . . . 86

2.4 .1 Testable restrictions $\ldots \ldots \ldots \ldots \ldots$

2.4.2 Extensions of the baseline model _ . . . . . . . . . 87

2.4.3 Extensions with more than two individual random variables . 89

2.4.4 Extension with overlapping samples and measurement error . 91

2.4 .5 Conditional analysis $\ldots \ldots \ldots \ldots$

2.5 Estimation . . . . . . . . . . . . . . . . . . . . . . 94

2.5 .1 Estimating $\phi_{V_{3}} \ldots \ldots \ldots \ldots \ldots \ldots$

2.5 .2 Estimating $f_{X_{1}, X_{2}} \ldots \ldots \ldots \ldots \ldots \ldots \ldots$

2.5 .3 Asymptotic properties . . . . . . . . . . . . . 100

2.6 Monte-Carlo Simulations . . . . . . . . . . . . . . . . . . . . . . . . . 101

2.6 .1 Design . . . . . . . . . . . . . . . . . . . . 101

2.6 .2 Simulation results . . . . . . . . . . . . . . . . . . . 102

2.6 .3 Truncation selection $\ldots \ldots \ldots$. . . . . . . . . . . 103

2.6.4 Practical implementation . . . . . . . . . . . . . . . . 104

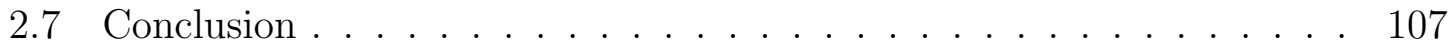

References . . . . . . . . . . . . . . . . . 108

C Appendix to Chapter 2 132

C.1 Proofs of Lemma 1 and Theorem 1 . . . . . . . . . . . . . . 132

C.2 Proof of Theorem $2 \ldots \ldots \ldots \ldots \ldots \ldots$

C.3 Selection Procedure for the Truncation Parameters $U$ and $T$. . . 139

C.4 Computational Algorithm for the Two-

Dimensional Fourier Inversion . . . . . . . . . . . . . . . 146 
3 Canadian Households' Payment Habits: 'Partnering Up' Aggregated Household Data and Individual Data 148

3.1 Introduction . . . . . . . . . . . . . . . . . . . . . . . . 148

3.2 Background . . . . . . . . . . . . . . . . . . . . . . . . . . . . 149

3.3 Survey Data on Methods of Payment . . . . . . . . . . . . . . 151

3.4 Motivating Exploratory Analysis _. . . . . . . . . . . . . 153

3.4.1 Intra-household influences with respect to methods of payment 154

3.4.2 Inter- and intra-household heterogeneity with respect to methods of payment . . . . . . . . . . . . . 157

3.5 Validity Assessment of the Methodological Approach . . . . . . . 159

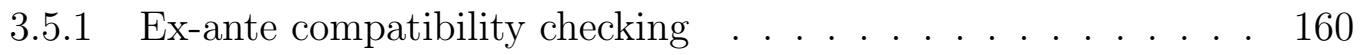

3.5.2 Real data performance evaluation using test data . . . . . . 162

3.6 Non-Parametric Estimation of Joint Payment Behavior within Couple Households by Sample Combination . . . . . . . . . . . . . 166

3.7 Conclusion . . . . . . . . . . . . . . . . . . . . . . . 168

References . . . . . . . . . . . . . . . . . 170 


\section{List of Tables}

1.1 Demographic characteristics of CTC users and non-users . . . . . . 30

1.2 Demographic characteristics of SVC users and non-users . . . . . . 31

1.3 Usage patterns of payment innovations in two consecutive years . . . 32

1.4 Representative samples, stayers and refreshers in the three-period panel 33

1.5 First-year demographic characteristics: attritors vs. stayers . . . . . . 34

1.6 First-year banking and payment characteristics: attritors vs. stayers . 35

1.7 Cash ratio regressions - three-year panel . . . . . . . . . . . . 36

1.8 Cash ratio regressions - two-year panels . . . . . . . . . . . . . . 37

A.1 Description of the estimated models . . . . . . . . . . . . . 44

B.1 Cash ratio regressions with attrition correction - CTC . . . . . . . 62

B.2 Cash ratio regressions with corrections - SVC ........ 63

B.3 Description of the two-year panel models . . . . . . . . . . . . . 64

B.4 Cash ratio regressions with CTC and SVC as regressors . . . . . . . 65

B.5 Item nonresponse in the three-year panel . . . . . . . . . . 66

B.6 Cash ratio regressions with item nonresponse correction - three-year

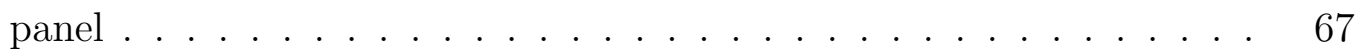

B.7 Cash ratio regressions under multiple-imputation for item nonresponse 68

2.1 Monte Carlo simulations - $V_{1}, V_{2} \sim N(0,1), V_{3} \sim N(0,0.1) \ldots \ldots$

2.2 Monte Carlo simulations - $V_{1}, V_{2} \sim N(0,1), V_{3} \sim N(0,0.5) \ldots \ldots$ 
2.3 Monte Carlo simulations - $V_{k} \sim$ Poisson(1) for $k=1,2,3 \ldots \ldots$

2.4 Monte Carlo simulations - $V_{1}, V_{2} \sim \operatorname{Poisson}(3), V_{3} \sim \operatorname{Poisson}(1) \ldots 115$

2.5 Monte Carlo simulations - $V_{1}, V_{2} \sim \operatorname{Poisson}(5), V_{3} \sim \operatorname{Poisson}(1) \ldots 116$

2.6 Monte Carlo simulations - $V_{k} \sim \operatorname{Gamma}(1,1)$ for $k=1,2,3 \ldots \ldots$

2.7 Monte Carlo simulations - $V_{k} \sim \operatorname{Gamma}(2,1)$ for $k=1,2,3 \ldots \ldots$

2.8 Optimal truncation estimates . . . . . . . . . . . . . . 119

3.1 Units of observation in the MOP and CFM surveys . . . . . . . . 172

3.2 Partners' joint probability of usage - CC and DC . . . . . . . . 173

3.3 Probability of usage conditional on partner's use - CC and DC . . . . 173

3.4 Regression analysis of partners' joint probability of usage - CC and DC 174

3.5 Regression analysis of self and partner methods of payment usage on personal cash management practices - CC and DC . . . . . . . . 175

3.6 Kolmogorov-Smirnov equality of distribution tests - single and couple households . . . . . . . . . . . . . . . . . 176

3.7 Kolmogorov-Smirnov equality of distribution tests - male and female heads of households . . . . . . . . . . . . . . . . . . . . . 177

3.8 Probability of payment methods usage and individual characteristics . 178

3.9 Frequency of payment methods usage and individual characteristics . 179

3.10 Share of payment methods usage and individual characteristics . . . . 180

3.11 Household demographics in the CFM and MOP samples . . . . . . . 181

3.12 Household head demographics in the CFM and MOP samples - couple households . . . . . . . . . . . . . . . . . . . . . . . . . . . . . . 182

3.13 Summary statistics - individual and aggregate variables . . . . . . . 183

3.14 Confidence intervals for sample means . . . . . . . . . . . . . . . . . 184

3.15 Confidence intervals for sample proportions . . . . . . . . . . . . . 185

3.16 Confidence intervals for sample variances . . . . . . . . . . . . . . 186

3.17 Summary statistics - test data . . . . . . . . . . . . 187 
3.18 Covariance and Pearson's correlation estimated on test data - CC and DC in volume, raw data . . . . . . . . . . . . . . . 187

3.19 Measures of association estimated on test data - CC and DC in volume, raw data . . . . . . . . . . . . . . . . . . . 188

3.20 Distance correlation estimated on test data - CC and DC in volume, raw data . . . . . . . . . . . . . . . . . . . . 189

3.21 Pearson's correlation estimates . . . . . . . . . . . . . . . . . . 190

3.22 Average mutual information estimates . . . . . . . . . . . . . . . 190

3.23 Distance correlation estimates . . . . . . . . . . . . . . . . 190 


\section{List of Figures}

1.1 Contactless card and terminal diffusion . . . . . . . . . . . . . 38

1.2 Stored-value cards in value and volume . . . . . . . . . . . . 38

1.3 Cash ratios of CTC and SVC users and non-users . . . . . . . . . . . 39

1.4 CTC, attrition probability versus the cash ratio in volume . . . . . . 40

1.5 SVC, attrition probability versus the cash ratio in volume . . . . . . . 41

B.1 Two-period panel with refreshment sample . . . . . . . . . . . . . 69

B.2 CTC, attrition probability versus cash ratio in volume - two-year panels 70

B.3 CTC, attrition probability versus cash ratio in value - two-year panels 71

B.4 SVC, attrition probability versus cash ratio in volume - two-year panels 72

B.5 SVC, attrition probability versus cash ratio in value - two-year panels 73

2.1 MISE estimates - normal distribution . . . . . . . . . . . . . . 120

2.2 MISE estimates - Laplace distribution _ . . . . . . . . . . . . . 121

2.3 MISE estimates - bivariate normal distribution . . . . . . . . . . . . . 122

2.4 MISE estimates - Poisson distribution . . . . . . . . . . . . . 123

2.5 MISE estimates - gamma distribution . . . . . . . . . . . . . . . 124

2.6 MISE estimates - geometric distribution . . . . . . . . . . . . 125

2.7 Contour plots of $\widehat{f}_{X_{1}, X_{2}}$ - normal case $-V_{3} \sim N(0,0.1)$ and $n=1,000 \quad$. 126

2.8 Contour plots of $\widehat{f}_{X_{1}, X_{2}}$ - normal case $-V_{3} \sim N(0,0.1)$ and $n=100$. . 127

2.9 Contour plots of $\widehat{f}_{X_{1}, X_{2}}$ - normal case $-V_{3} \sim N(0,0.5)$ and $n=1,000 \quad$. 128 
2.10 Contour plots of $\widehat{f}_{X_{1}, X_{2}}$ - normal case $-V_{3} \sim N(0,0.5)$ and $n=100 \quad \ldots 129$

$2.113 \mathrm{D}$ plot of $\widehat{f}_{X_{1}, X_{2}}-$ normal case $-V_{3} \sim N(0,0.1) \ldots \ldots \ldots$

$2.123 \mathrm{D}$ plot of $\widehat{f}_{X_{1}, X_{2}}-$ normal case $-V_{3} \sim N(0,0.5) \ldots \ldots \ldots 131$

3.1 Empirical CDFs - male and female heads of households . . . . . . . . 191

3.2 Estimated ISE obtained on test data . . . . . . . . . . . . . . . . . 192

3.3 Estimated marginal PMFs obtained on test data - DC in volume, raw

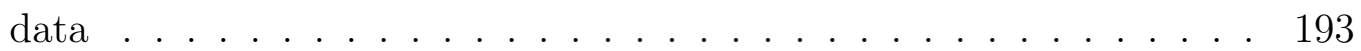

3.4 Estimated marginal PMFs obtained on test data - DC in volume, nonzero data . . . . . . . . . . . . . . . . . . . . . . . 194

3.5 Estimated joint density obtained on test data - DC in volume, residuals 195

3.6 Estimated marginal PMFs - CC in volume . . . . . . . . . . . . 196

3.7 Estimated marginal PMFs - SVC in volume . . . . . . . . . . . . 197

3.8 Estimated marginal PMFs - cash in volume . . . . . . . . . . . . 198

$3.9 \widehat{f}_{X_{F H}, X_{M H}}$ and $\widehat{f}_{X_{F H}} \widehat{f}_{X_{M H}}-$ CC in volume . . . . . . . . . . 199

$3.10 \widehat{f}_{X_{F H}, X_{M H}}$ and $\widehat{f}_{X_{F H}} \widehat{f}_{X_{M H}}-$ SVC in volume . . . . . . . . . . 200

$3.11 \widehat{f}_{X_{F H}, X_{M H}}$ and $\widehat{f}_{X_{F H}} \widehat{f}_{X_{M H}}$ - cash in volume . . . . . . . . . . 201 


\section{Chapter 1}

\section{Retail Payment Innovations and}

\section{Cash Usage: Accounting for}

\section{Attrition Using Refreshment}

\section{Samples}

\section{$1.1 \quad$ Introduction}

In the past 20 years, there has been a rapid transformation of retail payment systems. The share of cash, in terms of both volume and value, relative to non-cash payments has been decreasing; Amromin and Chakravorti (2009) document this trend in 13 developed countries for the period 1988-2003. However, a recent study by Bagnall et al. (2014) finds that cash still remains an important payment method at the pointof-sale (POS) across seven developed countries. The authors find that the cash share, in terms of volume, ranges from 40 percent for the United States to 80 percent for Austria. Further, cash still constitutes a non-trivial value share of payments at about

20 percent. One of the cited reasons for the continual use of cash is that it is used 
frequently for small-value transactions because of its speed, ease of use and wide acceptance; see Arango et al. (2015) and Wakamori and Welte (2012), who confirm this result for Canada. However, Arango et al. (2012) discuss a number of innovations in the retail payment market that are designed to mimic these attractive features of cash.

One type of innovations is the contactless feature based on near-field communication (NFC) technology. In Canada, almost all credit cards have a contactless feature and it is widely accepted by merchants. These contactless credit card (CTC) payments offer speed and convenience with tap-and-go and do not require a signature or personal identification number (PIN) verification for transactions below a certain value, typically $\$ 50$. Another type of payment innovation is stored-value or prepaid cards. Such cards, where monetary value is stored, can be grouped into two categories: (1) multi-purpose/open-loop cards, which are mostly offered by the main credit card providers, and (2) single-purpose/closed loop, which are issued by specific retailers. Our analysis will focus on single-purpose stored-value cards (SVC), as they are the major part of the market relative to multi-purpose cards. Also, SVC is designed as a substitute for cash, while multi-purpose cards appear to function chiefly as substitutes for credit cards especially for those who do not have sufficient credit ratings.

The introductions of these retail payment innovations are designed to ease transactions. Therefore, the main objective of this chapter is to estimate the impacts on cash usage of these two payment innovations, so as to inform policymakers whether cash can be displaced in the near future. Recent work by Rogoff (2014) espouses the need to eliminate cash for possible anonymous transactions in the grey economy. However, a large part of cash usage is for every day transactions and not illegal activities. Therefore, a viable payment system will be needed to undertake such a policy. Another rationale to understand the demand for cash is the potential decrease in 
seignoriage revenues. Fung et al. (2014b) discuss the implications of a reduction in cash usage: its impact on seigniorage revenues, and how it might affect a central bank's ability to conduct monetary policy and to promote financial stability. For example, Woodford (2003) argues that even if there was a complete displacement of cash by innovative payment instruments, monetary policy could continue to operate as long as the monetary authority could adjust bank reserves to control for the aggregate price level. Again, the practical implementation of such a policy is reliant on viable alternative methods of payment that are widely accepted. Finally, central banks need to understand the demand for cash as they are charged with efficient handling and distribution of cash; see Schmiedel et al. (2012). Moreover, market infrastructure participants such as card processors and merchants need to understand whether there is a demand for enhanced terminals.

However, there are few empirical estimates of the effects of these retail payment innovations on cash usage. Fung et al. (2014a) use the 2009 Bank of Canada Methodof-Payments (MOP) survey and find that the use of contactless credit cards results in a decrease in cash usage of about 13 and 14 percent in terms of volume and value of transactions, respectively. These results are based on cross-sectional data from 2009 and may be biased due to the presence of unobserved heterogeneity. In addition, there are few studies analyzing the uneven diffusion of innovations among both consumers and merchants, especially at the nascent stages. These patterns are known as S-curves: low rates of usage at the early stage, a turning point and then almost universal usage. Usually, industries such as the payment card industry are described as two-sided markets where network externalities matter; see Gowrisankaran and Stavins (2004) and Rysman (2007) who study the adoption and usage of an automated clearing house and credit cards, respectively.

The first contribution of this chapter is to use panel data from the Canadian Financial Monitor (CFM) to understand the impact of retail payment innovations on 
cash usage. We use the data from 2010, 2011 and 2012, as longitudinal data is essential to account for unobserved heterogeneity. Ignoring unobserved heterogeneity could confound and bias our estimates. For example, two persons who have similar observed characteristics might differ in unobserved dimensions, such as participation in the cash-intensive grey economy, causing a negative correlation between the payment innovation and unobserved heterogeneity. Using the ordinary least squares (OLS), the estimate would just be an average of estimates from these two users. Accounting for unobserved heterogeneity in the fixed-effect panel model might attenuate the omitted variable bias.

The second contribution is to address the high rate (about 50 percent) of nonignorable attrition. We build upon the literature of Hirano et al. (2001) and Bhattacharya (2008) to use refreshment samples to correct for potential attrition bias. Our methodological contribution is to provide identification of a three-period attrition probability function, and discuss how to control simultaneously for non-ignorable attrition and item nonresponse. We find that, in contrast to the findings of Fung et al. (2014a), SVC lowers cash usage in volume by about 2 percent, while no significant effects are found for CTC. These estimates illustrate that retail payment innovations have not yet made large inroads on cash usage. Therefore, central banks should be prudent in the design, production, and distribution of cash to meet the public demand.

The rest of this chapter is organized as follows. Section 1.2 offers an overview of cash and retail payment innovations in Canada, while Section 1.3 describes the CFM data used in the chapter. Section 1.4 presents the main model and discusses the impacts of attrition. Section 1.5 addresses the correction method for non-ignorable attrition via the refreshment sample. Estimation and results are presented in Section 1.6 while Section 1.7 concludes. 


\subsection{Overview of Cash and Retail Payment Innova- tions}

Cash is a ubiquitous payment method that is universally accepted. The end of cash has been predicted by many with the introduction of payment innovations, such as card-based payments. In one of the most comprehensive studies of payment diffusion and cash usage, Attanasio et al. (2002) highlight the role of Bancomat cards to withdraw cash from Automated Teller Machines (ATM) in Italy. Although ATM card adoption rates were 29 percent in 1991 and increased to 58 percent in 2004, cash did not disappear. Indeed, the work by Alvarez and Lippi (2009) highlights the complementary role of ATM cards on cash usage by theoretically modeling a "free random" withdrawal in the Baumol-Tobin model. Adding this feature allows their model to fit the micro data well and also reconciles why innovations do not lead to a large decrease in cash usage. Access to this new ATM technology leads to a reduction in cash holdings, but the number of cash withdrawals increases as the number of ATM machines increases. Further work by Alvarez and Lippi (2013) introduces the notion of large lumpy purchases that require cash into their theoretical model to rationalize the apparent cash usage, while Alvarez and Lippi (2015) show that consumers deplete their cash holdings before resorting to using their payment cards. This cash burns phenomena may be driven by the (non)-acceptance of payment cards, and therefore consumers hold cash as a precaution purpose as argued by Huynh et al. (2014).

Although cash usage remains high, a recent report by the Bank of International Settlements Committee on Payment and Settlement Systems (2012) studies the impact of innovations on the retail payment landscape. This report highlights Canada for the rollout of CTC. However, Fung et al. (2015) document that the ratio of bank notes outstanding to GDP has been relatively constant at about 3.5 percent in the last 30 years. One explanation for this trend, even though an overwhelming majority 
of consumers have access to payment cards (debit or credit cards), is that individuals also hold bank notes as a store of value and for precautionary purposes.

CTC were first introduced in Canada in 2006 (MasterCard PayPass) and 2007 (Visa payWave). Since NFC-enabled cards include a chip, the deployment of CTC and their associated terminals is closely related to the rollout of chip credit cards, which replace previous cards with magnetic stripes. In Canada, the migration to chip technology is spread out over eight years and culminated in 2015 with every credit card in the country containing a chip; see Arango et al. (2012). Since cards are converted without the cardholders' request, the adoption process of the contactless feature is considered passive or exogenous.

Figure 1.1 documents at the aggregate level the adoption of CTC for consumers and merchants. The graph clearly illustrates that an S-curve of adoption is at play but the question remains whether CTC has had an impact on cash transactions. Some evidence of this can be found in the recent survey results from detailed payment diaries in the 2013 Bank of Canada Methods-of-Payments Survey: Henry et al. (2015) highlight that cash has decreased in volume but not value, while at the same time CTC usage has increased dramatically in volume. To reconcile this difference, the authors find that the average transaction size of cash increased while that of CTC decreased. Further, the share of cash payments for transactions greater than $\$ 50$ increased. These results align with our findings on retail payment innovations: consumers are using them for smaller value transactions, and for speed and convenience.

In Canada, SVC adoption shows signs of steady growth as illustrated in Figure 1.2. In 2013, SVC accounted for about 45 million transactions and about 2,500 million in total value, with an average value of $\$ 55$. Consumers might use SVC instead of cash to avoid change in the form of coins, or may use cash to buy these cards as gifts, as their colloquial term is "gift cards." 


\subsection{The Canadian Financial Monitor}

The CFM is an annual survey of Canadian households, conducted by Ipsos Reid since 1999. It provides comprehensive information about household finances and includes demographics, banking habits, and household balance sheets (assets and liabilities). The annual size of the survey is approximately 12,000 households.

A module concerning methods of payment and cash usage was introduced in the 2009 CFM questionnaire on a trial basis and was revamped in 2010. We use the 2010, 2011 and 2012 data, since the payment-related questions are consistent and comparable. For a detailed description of the variables used in our study, refer to Appendix A.1. In the next few subsections we describe cash usage, the retail payment innovations, attrition and the refreshment sample.

\subsubsection{Cash usage}

We use two relative measures of cash usage based on volume and value constructed from the CFM data. For each household, the cash ratio in volume is the ratio of the total number of purchases paid in cash in the past month to the total number of all purchases (excluding bill payments) in the past month. The second measure, the cash share in value, is the ratio of the total value of cash purchases in the past month to the total value of all transactions (excluding bill payments) in the past month. Cash usage is more prevalent in terms of volume than value, given that cash is mainly used for small-value transactions. The median cash transaction in the CFM is about $\$ 20$, while it is about $\$ 40$ and $\$ 20$ for CTC and SVC, respectively.

Figure 1.3 provides the average cash ratios for users and non-users of payment innovations. For both innovations, the average non-user household pays around 37 percent of total volume purchases and 22 percent of total value purchases using cash. Those numbers are stable over the observation period. 
Innovation users spend relatively less cash than non-users, both in terms of volume and value. For SVC, the difference between user and non-user cash ratios is around 5 and 4 percentage points for volume and value, respectively. We observe much larger user/non-user discrepancies for CTC however, since the user's volume and value cash ratios are about 11 percentage points smaller than the non-user's.

\subsubsection{Retail payment innovations}

Tables 1.1 and 1.2 provide some descriptive statistics on who uses these retail payment innovations. In general, households that use payment innovations tend to have a larger family size and younger family heads, and live in large cities. CTC and SVC users are also more likely to be employed and own their home, and earn higher household income.

Table 1.3 reports the usage patterns of payment innovations in two consecutive years in the 2010-12 three-year balanced panel. Innovations have different penetration rates in the retail payment market. CTC has a smaller presence in the market, since 74 percent of the households never used CTC during this period while only 49 percent never used SVC. They also differ in terms of the persistence of usage, which we assess as the ratio between the always-users $(\mathrm{U} ; \mathrm{U})$ and the sum of always-users and stopusers (U;N-U) in Table 1.3. CTC users are relatively more persistent, with about 70 percent of users in a given year continuing to use in the following year. This rate is about 50 percent for SVC. Finally, the users' switching rates can be measured as the proportion of households that either: (1) use but stop using or (2) do not use and start using $(\mathrm{N}-\mathrm{U} ; \mathrm{U})$ in two consecutive years. This rate is higher for SVC with 33 percent, compared to 13 percent for CTC.

Table 1.3 also provides information about multiple payment innovation users: we observe that 40 percent of CTC users also use SVC, while only about 20 percent of SVC users also use CTC. Among the households that use one of the two innovations 
(35 percent of all households), only 16 percent use both. Tables 1.1 and 1.2 further demonstrate that CTC and SVC users differ on some aspects. CTC users earn higher income and are slightly more likely to live in large urban areas and own their home relative to SVC users. CTC users are also much more likely to live in the province of Quebec and much less likely to live in Western provinces than SVC users.

\subsubsection{Attrition and refreshment sample}

The CFM survey has a sampling and weighting procedure to obtain annual representations of the Canadian population. Past participants are re-interviewed in following years, so that the data have a panel dimension (a household can receive only one CFM questionnaire in each 12-month period). However, the survey is voluntary, and Table 1.4 indicates an annual attrition rate of 50 percent. Among the 11,695 households observed in 2010, 49 percent (5,699 households) participated again in 2011, while 33 percent (3,853 households) participated again in both 2011 and 2012.

To handle attrition, the survey company recruits new respondents to the CFM to maintain a constant yearly sample size and ensure that the cross-section is representative according to seven main demographic and geographic variables listed in Table 1.1. These characteristics are measured at the time of entry into the sampling frame, so that they are considered as time-invariant over 2010-2012.

In the random refreshment samples considered in Hirano et al. (2001) and Bhattacharya (2008), new sampling units are randomly drawn from the population, and hence are themselves representative. In contrast, the CFM refreshment samples are drawn so that the combination of the stayers and these additional units is representative in a given year. These are sometimes called top-up refreshment samples, as described in Cheng and Trivedi (2015). The crucial feature of the CFM data is that in each period the cross-sectional sample is representative of the Canadian population according to seven main demographic and geographic variables. 


\subsection{Panel Data Estimation and Attrition}

We utilize the panel dimension of the CFM survey over the years 2010 to 2012. The standard panel data model with unobserved heterogeneity $\left(\alpha_{i}\right)$ is:

$$
C R_{t i}=\alpha_{i}+\beta P I_{t i}+D e m o_{t i} \gamma+u_{t i}
$$

where $C R$ denotes the cash ratio, $P I$ is a binary variable denoting the use of a payment innovation, Demo contains demographic variables described in Appendix $\mathrm{A}$, and $u_{t i}$ is the idiosyncratic error term. The parameter of interest, $\beta$, measures the effect of retail payment innovations on household cash usage. ${ }^{1}$

Ignoring $\alpha_{i}$ will introduce an omitted variable bias in the standard OLS or other cross-sectional estimators. One popular approach, commonly known as the randomeffects method, assumes that $\alpha_{i}$ is independent of all right-hand side variables and that its conditional distribution belongs to a known parametric family. A less restrictive alternative is to difference out $\alpha_{i}$ to obtain:

$$
\Delta C R_{t i}=\beta \Delta P I_{t i}+\Delta D e m o_{t i} \gamma+\Delta u_{t i} .
$$

This would reduce potential concerns about endogeneity due to the omitted variable.

If $\Delta P I$ is correlated with $\Delta u$, an instrument for $\Delta P I$ will be needed. However, we know that CTC and SVC tend to be passively adopted and thus exogenous. In the case of CTC banking institutions send customers the new card upon renewal, while SVC tend to be given as gifts. If there is any active adoption, PI should be correlated with either observables or unobserved heterogeneity, but not the idiosyncratic errors.

However, the fixed-effect approach does not solve the problem of attrition. Recall

\footnotetext{
${ }^{1}$ We estimate the effects of payment innovations in two separate regressions: one for CTC, and the other for SVC. As an alternative, we also estimate their effects in a single model with two dummies for CTC and SVC, and results are similar to the previous separate regressions. Results are available in Technical Appendix.
} 
the CFM suffers from a 50 percent attrition rate. Equation (1.2) can only be estimated on units that remain in the sample during two consecutive periods (stayers). Fitzgerald et al. (1998) state that "the most potentially damaging threat [...] to the value of panel data is the presence of biasing attrition." Depending on the form of the attrition process at work, these fixed-effect estimates may be biased. Note that in this paper we assume away population attrition; see Kim (2012).

Consider the following moment condition implied by Equation (1.2):

$$
E\left[\phi\left(z_{t-1}, z_{t}, \beta\right) \mid x_{t-1}, x_{t}\right]=0
$$

where $\phi(\cdot)=\Delta C R_{t}-\beta \Delta P I_{t}-\Delta D e m o_{t} \gamma, x_{t}=\left(P I_{t}, D e m o_{t}\right)$ and $z_{t}=\left(C R_{t}, x_{t}\right)$. We define the conditional probability of attrition as:

$$
\operatorname{Pr}\left(S_{t-1, t}=0 \mid z_{t-1}, z_{t}, v\right) \equiv 1-g\left(z_{t-1}, z_{t}, v\right)
$$

where $S_{t-1, t}=0$ for attritors and $S_{t-1, t}=1$ for stayers (households observed in year $t-1$ participating again in year $t$ ), and $v$ are time-invariant regressors. By the law of iterated expectations, (1.3) and (1.4) imply that

$$
E\left[\frac{\phi(\cdot)}{g(\cdot)} \mid S_{t-1, t}=1, x_{t-1}, x_{t}\right]=0
$$

if $E\left(S_{t-1, t} \mid x_{t-1}, x_{t}\right)>0$ for all $x_{t-1}, x_{t}$.

There are two types of attrition process such that (1.5) implies $E\left[\phi(\cdot) \mid S_{t-1, t}=1\right.$, $\left.x_{t-1}, x_{t}\right]=0$, so that considering only the stayers will not bias the estimates and thus attrition is ignorable. The first is the well-known missing-completely-at-random (MCAR) case, when the attrition mechanism is independent of all other variables in the model: $S_{t-1, t} \perp C R_{t-1}, C R_{t}, x_{t-1}, x_{t}$. The second, termed completely ignorable by Bhattacharya (2008), is when $S_{t-1, t} \perp C R_{t-1}, C R_{t} \mid x_{t-1}, x_{t}$. 
Tables 1.5 and 1.6 provide first-year summary statistics for three-year stayers and attritors. ${ }^{2}$ These tabulations reveal some significant differences between stayers and attritors with the last two columns reporting $F$-statistics and p-values. Attritor households tend to have younger family heads, are more likely to rent and live in an urban area. They are also more likely to be employed and live in a larger household with higher household income. Table 1.6 shows that stayers and attritors also frequently differ in their banking and payment characteristics. Therefore, we find evidence to reject the null hypothesis that attrition is MCAR. In the next section, we explore how to address non-ignorable attrition.

\subsection{Correcting for Non-ignorable Attrition}

The moment condition (1.5) suggests that, when attrition is neither MCAR nor completely ignorable, using the balanced panel (i.e. conditioning on stayers) requires that we identify the survival probability function $g(\cdot)$. Earlier work can be categorized based on the assumptions made on the attrition mechanism. Rubin (1976) and Little and Rubin (1987) discuss the missing-at-random (MAR) assumption (also called selection-on-observables) that allows the probability of attrition to depend on lagged but not contemporaneous variables. Another model, sometimes referred to as HW in reference to Hausman and Wise (1979), allows attrition to depend on contemporaneous variables only. Because contemporaneous variables are not observed for attritors, this is a case of selection-on-unobservables.

Fitzgerald et al. (1998) and Section 19.9 in Wooldridge (2010) provide an excellent summary for handling either MAR or HW attrition. Wooldridge (2002) discusses using inverse probability weighting to correct for MAR attrition, while Nijman and Verbeek (1992), Vella and Verbeek (1999), Das (2004) and Cheng and Trivedi (2015)

\footnotetext{
${ }^{2}$ Sample sizes differ because, while the seven basic demographics in Table 1.5 are always completely observed, other variables suffer from item nonresponse. In Subsection 1.5.3 we show that our methodology can be extended to correct for both attrition and item nonresponse.
} 
provide various two-step estimators in order to handle HW attrition.

However, the availability of additional data can allow for more flexible attrition processes. Nevo (2002) and Nevo (2003) utilize population moments, and Davezies and DHaultfouille (2011) use instrumental variables. In this chapter, we follow Hirano et al. (2001) and Bhattacharya (2008) by exploiting refreshment samples. In Subsection 1.5.2, we first recall their identification results in the two-period panel situation, then extend it to the three-period case. In Subsection 1.5.3, we further discuss how to deal simultaneously with attrition and item nonresponse.

\subsubsection{Identification of attrition function without refreshment}

Without a refreshment sample, the survival function $g(\cdot)$ can be specified with either lagged variables (MAR) or contemporaneous variables (HW), but not both. Let us specify $g(\cdot)$ as a single-index model $g(k(\cdot))$ where $g: \mathbb{R} \rightarrow[0,1] .^{3}$

In the two-period panel, the MAR survival probability $g\left(k\left(z_{1}, v\right)\right)$ is directly identified by $E\left[S_{12} \mid z_{1}, z_{2}, v\right]=E\left[S_{12} \mid z_{1}, v\right]$, which is estimable using the unbalanced panel only. On the contrary, in the case of HW, attrition depends on unobservables. Note that $E\left[S_{12} \mid z_{2}, v\right]=g\left(k\left(z_{2}, v\right)\right)$ can be identified from $E\left[1 / g\left(k\left(z_{2}, v\right)\right) \mid z_{1}, v\right]=$ $1 / E\left[S_{12} \mid z_{1}, v\right]$ under the assumption that $E\left[S_{12} \mid z_{1}, z_{2}, v\right]=E\left[S_{12} \mid z_{2}, v\right]$. These results can be easily extended to the three-period case.

Notice that it is not possible to test either MAR or HW using the unbalanced panel only as discussed in Nicoletti (2006), while a refreshment sample could be used to test either MAR or HW assumptions, as discussed in Hirano et al. (2001). Most importantly, the extra information provided by the refreshment sample can also be exploited to identify a more general survival function.

\footnotetext{
${ }^{3}$ We assume the function $g$ is known (i.e., logit or probit), but Horowitz and Mammen (2004) show that it can be estimated by the penalized least-squares estimator.
} 


\subsubsection{Identification of attrition function with refreshment}

\subsubsection{Identification of a two-period attrition function with refreshment}

In the context of a two-period panel, Hirano et al. (2001) show that the refreshment data can help identify a class of models that generalizes MAR and HW by allowing the survival function to depend both on lagged and contemporaneous variables. These functions are of the form $g\left(k\left(z_{1}, z_{2}, v\right)\right)=g\left(k_{0}(v)+k_{1}\left(z_{1}, v\right)+k_{2}\left(z_{2}, v\right)\right)$, where $g(\cdot)$ is a known $\mathrm{CDF}$ and functions $k_{j}(\cdot), j=0,1,2$ are non-parametrically identified up to a location normalization. This class of model is referred to as additive non-ignorable $(\mathrm{AN})$, as it rules out any interaction between $z_{1}$ and $z_{2}$. The AN model nests the MAR and HW models as special cases. Bhattacharya (2008) rewrites Hirano et al. (2001)'s identifying integral equations as the equivalent moment restrictions

$$
\begin{aligned}
& E\left[\frac{S_{12}}{g\left(k_{0}(v)+k_{1}\left(z_{1}, v\right)+k_{2}\left(z_{2}, v\right)\right)}-1 \mid z_{1}, v\right]=0, \text { a.e. } z_{1} \text { and } v \\
& E\left[\frac{S_{12}}{g\left(k_{0}(v)+k_{1}\left(z_{1}, v\right)+k_{2}\left(z_{2}, v\right)\right)}-1 \mid z_{2}, v\right]=0, \text { a.e. } z_{2} \text { and } v
\end{aligned}
$$

While Equation (1.6) is identifiable from the unbalanced panel, this is not the case for Equation (1.7), which requires a representative sample of the population in period 2. This identification result holds regardless of whether the representative sample is provided by a random refreshment sample as in Hirano et al. (2001) and Bhattacharya (2008), or the combination of the stayers and a top-up refreshment sample as in our case.

\subsubsection{Identification of a three-period attrition function with refresh- ment}

Our data consist of three years, so we now discuss the case of a three-period attrition function that is allowed to depend on the $z_{1}, z_{2}$ and $z_{3}$ variables. $S_{12} S_{23}=1$ indi- 
cates a unit observed in the initial sample (period 1) that survives in both periods 2 and 3 (three-year stayers). An extension of Hirano et al. (2001), also discussed in Bhattacharya (2006), yields the non-parametric identification of a survival probability $\operatorname{Pr}\left(S_{12} S_{23}=1 \mid z_{1}, z_{2}, z_{3}, v\right)$ of the form $g\left(k_{0}(v)+k_{1}\left(z_{1}, v\right)+k_{2}\left(z_{2}, v\right)+k_{3}\left(z_{3}, v\right)\right)$. A formal statement is given below. This result relies on the availability of representative samples in each period, which is equivalent to knowing the marginal densities of $z_{1}, z_{2}$ and $z_{3}$.

Let $\kappa$ denote a generic function and $\kappa_{0}$ the true function. Identification amounts to showing that if any function $\kappa$ satisfies the moment conditions listed in (ii) of Proposition 1 , then $\kappa$ equals the true function $\kappa_{0}$.

Proposition 1. If (i) conditional on each value $v$ in its support, the support $\mathcal{Z}_{1}(v) \times$ $\mathcal{Z}_{2}(v) \times \mathcal{Z}_{3}(v)$ of $Z_{1}, Z_{2}$ and $Z_{3}$ is not a lower-dimensional subspace of $\mathcal{R}^{3 \times \operatorname{dim}\left(Z_{1}\right)}$; (ii)

$$
\begin{aligned}
& E\left[\frac{S_{12} S_{23}}{g\left(k_{0}(v)+k_{1}\left(z_{1}, v\right)+k_{2}\left(z_{2}, v\right)+k_{3}\left(z_{3}, v\right)\right)}-1 \mid z_{1}, v\right]=0 \text { a.e. } z_{1}, v \\
& E\left[\frac{S_{12} S_{23}}{g\left(k_{0}(v)+k_{1}\left(z_{1}, v\right)+k_{2}\left(z_{2}, v\right)+k_{3}\left(z_{3}, v\right)\right)}-1 \mid z_{2}, v\right]=0 \text { a.e. } z_{2}, v \\
& E\left[\frac{S_{12} S_{23}}{g\left(k_{0}(v)+k_{1}\left(z_{1}, v\right)+k_{2}\left(z_{2}, v\right)+k_{3}\left(z_{3}, v\right)\right)}-1 \mid z_{3}, v\right]=0 \text { a.e. } z_{3}, v ;
\end{aligned}
$$

(iii) $g(\kappa)$ is strictly increasing over the range of $\kappa, \lim _{a \rightarrow-\infty} g(a)=0=1-\lim _{a \rightarrow \infty} g(a)$; and (iv) for each $v$, there exists $\bar{z}_{1}(v) \in \mathcal{Z}_{1}(v), \bar{z}_{2}(v) \in \mathcal{Z}_{2}(v)$ and $\bar{z}_{3}(v) \in \mathcal{Z}_{3}(v)$ such that $k_{1}\left(\bar{z}_{1}(v), v\right)=k_{2}\left(\bar{z}_{2}(v), v\right)=k_{3}\left(\bar{z}_{3}(v), v\right)=0$, then $\kappa=\kappa_{0}$ w.p.1.

As argued in Hirano et al. (2001), the problem of identifying the survival probability can be cast under the empirical likelihood framework, where we estimate the three-period joint distributions $f_{1,2,3}\left(z_{1}, z_{2}, z_{3} \mid v\right)$ as the one closest to the joint distribution $f_{1,2,3}\left(z_{1}, z_{2}, z_{3} \mid S_{12} S_{23}=1, v\right)$, subject to the restrictions imposed by the data.

Proposition 1 rules out interactions among $z_{1}, z_{2}$ and $z_{3}$ in the three-period at- 
trition function, but it is possible that the latter is over-identified. For a binary outcome $Y$, Deng et al. (2013) demonstrate just-identification for the joint distribution of $\left(Y_{1}, Y_{2}, Y_{3}, S_{12}, S_{23}\right)$ by counting the number of cells in the contingency table and the number of parameters in the saturated model. However it is not obvious how to obtain the just-identification of the attrition function in the case where the outcome variable $Y$ is continuous. We leave it for future research.

\subsubsection{Identification with both attrition and item nonresponse}

We now consider the case where, in the unbalanced panel and refreshment samples, a subset of observations suffer from item nonresponse (for a unit $i$ observed in a given sample at time $t$, at least one $z_{t i}$ variable is missing). Let us define $C_{t i}$ as a binary variable indicating complete observations. To simplify notation, we further

define $C_{i} \equiv C_{1 i} C_{2 i} C_{3 i}$. If both attrition and item nonresponse are either MCAR or completely ignorable, the estimation of the main model on the subsample of stayers with complete observations does not require any correction (more precisely, the condition is $\left.C S_{12} S_{23} \perp y_{t-1}, y_{t} \mid x_{t-1}, x_{t}\right)$. If both selection mechanisms are non-ignorable, Equation (1.3) must be modified to:

$$
E\left[\frac{\phi(\cdot)}{g^{*}\left(k\left(z_{1}, z_{2}, z_{3}, v\right)\right)} \mid C=1, S_{12} S_{23}=1, x_{2}, x_{3}\right]=0
$$

where $\operatorname{Pr}\left(C S_{12} S_{23}=1 \mid \cdot\right)=g^{*}(\cdot)$.

If the $z_{t}$ variables observed in the data are not representative due to item nonresponse, moments of the form

$$
E\left[\left(\frac{C S_{12} S_{23}}{g^{*}\left(k_{0}(v)+k_{1}\left(z_{1}, v\right)+k_{2}\left(z_{2}, v\right)+k_{3}\left(z_{3}, v\right)\right)}-1\right) \mid z_{t}, v\right]=0
$$

are not identified. They must be transformed to: 


$$
E\left[\left(\frac{C S_{12} S_{23}}{g^{*}\left(k_{0}(v)+k_{1}\left(z_{1}, v\right)+k_{2}\left(z_{2}, v\right)+k_{3}\left(z_{3}, v\right)\right)}-1\right) \frac{C_{t}}{h_{t}(\cdot)} \mid z_{t}, v\right]=0,
$$

where $\operatorname{Pr}\left(C_{t}=1 \mid \cdot\right)=h_{t}(\cdot)$. The probability function $g^{*}$ (under a quasi-separability restriction) is identified provided that $h_{t}$ is identified, for $t=1,2,3$.

Let us define $z=\left(z_{1}, z_{2}, z_{3}\right)$ and $w=\left(w_{1}, w_{2}, w_{3}\right)$ for some variables $w_{t}$ exempt from item nonresponse and distinct from $z_{t}$. In Equation (1.10), we need for $t=3$ to identify $\operatorname{Pr}\left[C_{3}=1 \mid C S_{12} S_{23}, z, w, v\right] \equiv h_{3}\left(C S_{12} S_{23}, z, w, v\right)$. Let us specify $h_{3}($.$) as$ a single-index model $h\left(l_{3}().\right)$ where $h: \mathbb{R} \rightarrow[0,1]$. Recall that $z_{i t}$ denotes a vector of variables. $C_{i t}$ is defined such that $C_{i t}=1$ if $z_{i t}$ is completely observed (no item nonresponse), and $C_{i t}=0$ otherwise. In the sequel, we employ the same terminology used to designate attrition models, but redefine the MAR, HW and AN assumptions to fit the context of missingness due to item nonresponse.

Case 1. Item nonresponse is MAR: The MAR assumption is selection-onobservables. In our context, it can be defined as:

$$
\begin{aligned}
\operatorname{Pr}\left[C_{3}=1 \mid C S_{12} S_{23}, z, w, v\right] & =\operatorname{Pr}\left[C_{3}=1 \mid w_{3}, v\right] \\
& =h\left(l_{3}\left(w_{3}, v\right)\right)
\end{aligned}
$$

where $w_{3}$ is exempt from item nonresponse. Hence we can directly identify/estimate $h\left(l_{3}\left(w_{3}, v\right)\right)$ from the third period cross-section data (i.e. a representative sample of $\left\{C_{3}, w_{3}, v\right\}$ is observed).

Case 2. Item nonresponse is HW: The HW assumption is selection-on-unobservables. 
In our context, it can be written as

$$
\begin{aligned}
\operatorname{Pr}\left[C_{3}=1 \mid C S_{12} S_{23}, z, w, v\right] & =\operatorname{Pr}\left[C_{3}=1 \mid z_{3}, v\right] \\
& =h\left(l_{3}\left(z_{3}, v\right)\right)
\end{aligned}
$$

where $z_{3}$ is not completely observed if $C_{3}=0$. In order to identify $h\left(l_{3}\left(z_{3}, v\right)\right)$, Davezies and D'Haultfoeuille (2011)'s instrumental variable method can be implemented. Identifying moments are

$$
E\left[\frac{C_{3}}{h\left(l_{3}\left(z_{3}, v\right)\right)}-1 \mid w_{3}^{I V}, v\right]=0
$$

where the instrumental variable $w_{3}^{I V}$ is exempt from item nonresponse with rank condition to be satisfied. Notice that we can not allow for $h_{3}($.$) to depend on z_{t}$ for $t \neq 3$ because $C_{3}=1$ does not guarantee that $z_{1}$ or $z_{2}$ is observed.

Case 3. Item nonresponse is $\mathbf{A N}$ : The AN assumption is the mixture of the above MAR and HW assumptions. In our context, it can be defined as:

$$
\begin{aligned}
\operatorname{Pr}\left[C_{3}=1 \mid C S_{12} S_{23}, z, w, v\right] & =\operatorname{Pr}\left[C_{3}=1 \mid w_{3}, z_{3}, v\right] \\
& =h\left(l_{3}\left(w_{3}, z_{3}, v\right)\right)
\end{aligned}
$$

where $z_{3}$ has item nonresponse, but not $w_{3}$. In order to identify the function $h\left(l_{3}\left(w_{3}, z_{3}, v\right)\right)$ $=h\left(l_{0}(v)+l_{w}\left(w_{3}, v\right)+l_{z}\left(z_{3}, v\right)\right)$, we use the moments

$$
\begin{aligned}
& E\left[\frac{C_{3}}{h\left(l_{0}(v)+l_{w}\left(w_{3}, v\right)+l_{z}\left(z_{3}, v\right)\right)}-1 \mid w_{3}, v\right]=0 \\
& E\left[\frac{C_{3}}{h\left(l_{0}(v)+l_{w}\left(w_{3}, v\right)+l_{z}\left(z_{3}, v\right)\right)}-1 \mid w_{3}^{I V}, v\right]=0
\end{aligned}
$$

where both $w_{3}$ and $w_{3}^{I V}$ are exempt from item nonresponse with rank condition to be satisfied. 


\subsection{Estimation and Results}

This section discusses the estimation methodology and presents the empirical results.

\subsubsection{Estimation and Inference}

Bhattacharya (2008) employs a sieve minimum distance (SMD) method proposed in Ai and Chen (2003) to jointly estimate $\beta$ and the survival function $g(\cdot)$. Since different conditioning variables are used in the different conditional moments, we use the methodology of Ai and Chen (2007). Here we outline the SMD estimator for the conditional moments $M_{2,3}^{20}, M_{1}^{21}, M_{2}^{22}$ and $M_{3}^{23}$ with $\delta \equiv(\beta, \kappa)$ in Appendix A.2. Further refer to this Appendix for the sets of conditional moments used in our estimations of models $\mathcal{M}_{N C}^{1}, \mathcal{M}_{A N}^{1}, \mathcal{M}_{N C}^{2}$ and $\mathcal{M}_{A N}^{2}$. Denote by $n$ the total number of households in the three-period panel, $n_{t}$ the number of units in each period $t$ for $t=1,2,3$, and $n_{123}$ the number of stayers in the three-period balanced panel. Following Ai and Chen (2007), we use sieves to approximate (i) conditional moments based on conditioning variables $\left(x_{2}, x_{3}\right)$ and $\left(z_{t}, v\right)$ where $t=1,2,3$; and (ii) the unknown function $\kappa$.

Let $\left\{p_{0 l}\left(x_{2}, x_{3}\right), p_{1 l}\left(z_{1}, v\right), p_{2 l}\left(z_{2}, v\right), p_{3 l}\left(z_{3}, v\right)\right\}_{l=1, \ldots, K_{n}}$ be known sieve functions whose number $K_{n}$ grows slowly with the sample size. Also for $t=1,2,3$ define:

$$
\begin{array}{ll}
p_{0}^{K_{n}}\left(x_{2 i}, x_{3 i}\right)=\left\{S_{12 i} S_{23 i} p_{0 l}\left(x_{2 i}, x_{3 i}\right)\right\}_{l=1, \ldots, K_{n}} & \text { and } P_{0}=\left\{p_{0}^{K_{n}}\left(x_{2 i}, x_{3 i}\right)\right\}_{i=1,2, \ldots, n} \\
p_{t}^{K_{n}}\left(z_{t i}, v_{i}\right)=\left\{R_{t i} p_{t l}\left(z_{t i}, v_{i}\right)\right\}_{l=1, \ldots, K_{n}} & \text { and } P_{t}=\left\{p_{t}^{K_{n}}\left(z_{t i}, v_{i}\right)\right\}_{i=1,2, \ldots, n}
\end{array}
$$

and let

$$
\begin{aligned}
& \widehat{m}_{0}\left(x_{2 j}, x_{3 j}, \delta\right) \equiv \sum_{i=1}^{n} S_{12 i} S_{23 i} \frac{\phi\left(z_{2 i}, z_{3 i}, \beta\right)}{g\left(\kappa_{n}\left(z_{1 i}, z_{2 i}, z_{3 i}, v_{i}\right)\right)} p_{0}^{K_{n}}\left(x_{2 i}, x_{3 i}\right)^{\prime}\left(P_{0}^{\prime} P_{0}\right)^{-1} p_{0}^{K_{n}}\left(x_{2 j}, x_{3 j}\right), \\
& \widehat{m}_{t}\left(z_{t j}, v_{j}, \delta\right) \equiv \sum_{i=1}^{n} \frac{n_{t}}{n_{1}} R_{t i} \frac{S_{12 i} S_{23 i}}{g\left(\kappa_{n}\left(z_{1 i}, z_{2 i}, z_{3 i}, v_{i}\right)\right)} p_{t}^{K_{n}}\left(z_{t i}, v_{i}\right)^{\prime}\left(P_{t}^{\prime} P_{t}\right)^{-1} p_{t}^{K_{n}}\left(z_{t j}, v_{j}\right)-1,
\end{aligned}
$$


where $\kappa_{n}$ is the linear sieve approximations of $\kappa$, and $R_{t i}$ denotes whether a unit $i$ belongs to the representative sample in period $t$, for $t=1,2,3$. Notice that we are writing the estimated conditional moments under the common index $i=1, \ldots n$, which allows us to exactly follow Ai and Chen (2007) for calculating the asymptotic variance. The SMD estimators of $\widehat{\delta} \equiv(\widehat{\beta}, \widehat{\kappa})$ are obtained by minimizing

$$
\frac{1}{n} \sum_{j=1}^{n}\left\{\frac{n}{n_{123}} \widehat{m}_{0}\left(x_{2 j}, x_{3 j}, \delta_{n}\right)^{2}+\sum_{t=1}^{3} \frac{n}{n_{t}} \widehat{m}_{t}\left(z_{t j}, v_{j}, \delta_{n}\right)^{2}\right\} .
$$

For the inference, we follow the approach by Ai and Chen (2007) and Bhattacharya (2008) to derive the standard errors of the finite dimensional parameter estimates $\widehat{\beta}$. Details can be found in Chen et al. (2014). ${ }^{4}$

\subsubsection{Impact of Retail Payment Innovations}

This section reports two main findings: first, the cross-sectional estimates of the

payment innovation impact $(\hat{\beta})$ are larger in absolute magnitude for CTC in relation to the panel estimates. This difference justifies the importance of controlling for unobserved heterogeneity. Second, $\hat{\beta}$ is larger in absolute magnitude for SVC (in the case of cash ratio in value) if we do not account for non-ignorable attrition. These results show that failing to correct for unobserved heterogeneity and non-ignorable attrition will lead to overestimation of the impact of retail payment innovations on cash usage. We will discuss the sources and mechanisms behind these biases in this section.

\subsubsection{Cross-sectional and panel data analysis}

The outcome variable, the cash ratio, is measured in terms of value or volume and we consider two types of payment innovations, CTC and SVC. Therefore, four different

\footnotetext{
${ }^{4}$ Details on how to derive and estimate the asymptotic variance of $\hat{\beta}$ (in the two-period panel case) and a description of our practical implementation can be found in Technical Appendix.
} 
sets of parameters are estimated. To understand the importance of controlling for unobserved heterogeneity, the estimates obtained on cross-sectional or pooled data can be compared with the first-differencing (FD) estimates obtained on the three-year balanced panel without correcting for attrition. Results for the estimated parameters are summarized in Columns 1 to 6 of Table 1.7.

For CTC, estimates obtained on cross-sectional or pooled data are all highly significant. The estimated negative impacts of CTC on cash usage range between 8 and 10 per cent for both volume and value in our 2010-12 CFM study. These results are comparable to Fung et al. (2014a) estimates of 13 and 14 percent obtained on the 2009 cross-section MOP survey.

The balanced panel FD estimates of CTC is insignificant, which indicates that unobserved heterogeneity drives the results obtained on cross-sectional data. Therefore, ignoring unobserved heterogeneity will lead to overstatement in the impact of CTC on cash usage. The estimated impact of SVC for cash in value also loses statistical significance once unobserved heterogeneity is differentiated out. For cash in volume, the consequences of controlling for unobserved heterogeneity are smaller. The parametric and non-parametric panel estimates are around 2 percent and statistically significant.

\subsubsection{Effects of correcting for attrition}

The panel coefficient estimates are obtained for each model with and without correction for attrition, as summarized in Columns 5 to 7 of Table 1.7. There are many potential channels through which attrition correction might influence the estimation. In what follows, we put forward three main mechanisms that seem to explain our results.

First, identification of $\beta$ relies on switchers $(\Delta P I \neq 0)$ in the panel data, and the

accuracy of $\hat{\beta}$ is positively related to the number of switchers. The attrition correction mechanism can compensate for the small proportion of switchers. If switchers receive 
larger weights than non-switchers, the impact of switchers is further augmented by the attrition correction. Second, within the switchers, new-users $(\Delta P I=1)$ tend to be associated with negative changes in cash ratios $(\Delta C R<0)$, while stop-users $(\Delta P I=-1)$ are more likely to experience positive changes in cash ratio $(\Delta C R>0)$. Hence the magnitude and sign of $\beta$ are also driven by the size of new-users and their range for $\triangle C R$. Attrition correction might then impact the estimation by changing the relative importance of new-users and stop-users in the switchers' sample. Finally, the estimate of $\beta$ is obtained using two different subsamples, the 2010-11 two-year balanced panel (used for $M_{1,2}^{10}$ ) and the three-year balanced panel (used for $\left.M_{2,3}^{20}\right)$. Attrition correction can influence the results by weighting the two subsamples differently. For brevity we focus only on the volume for CTC and SVC, and Figures 1.4 and 1.5 illustrate these three mechanisms for these two cases.

Panel estimates of the impact of CTC are not significant, with or without attrition correction. It is clear from Figure 1.4 that this result is mainly driven by a relatively small number of switchers (either new-users or stop-users), associated with small changes in cash ratios. We observe both a small extensive margin with only 13 percent of households being switchers, and a relatively small intensive margin with the support of $\Delta C R$ being narrow and centered around zero. As a result, the inverse probability-weighting offered by the attrition correction, $1 / g(\cdot)$, does not affect $\hat{\beta}$.

For SVC, estimates obtained without correcting for attrition are negative and significant with a 2 percent reduction in the cash ratio in volume, but insignificant for the cash ratio in value. As mentioned previously, SVC are characterized by higher switching rates than CTC. The negative sign of our volume estimate is mainly due to the decreased cash usage by new-users, who account for more than half of the switchers. In the value case, the impact of new-users is offset by the stop-users associated with a negatively centered intensive margin; see Figure 1.5. Attrition correction comes into play by weighting stop-users relatively more than new-users. 
The resulting estimate for cash in value is reduced, while that for volume is still around 2 percent. In brief, after controlling for unobserved heterogeneity and attrition, only SVC is found to have a significant impact on cash usage: on average, the use of SVC by at least one person in the household decreases the number of purchases paid in cash by approximately 2 percent.

As evidenced by sample size differences across the descriptive Tables 1.5 and 1.6, the CFM data also suffers from item nonresponse. While several basic demographics (used as sampling targets) are always completely observed, other variables suffer from item nonresponse. In Subsection 5.3 we show that our reweighting methodology can be extended to correct for both attrition and item nonresponse. The last column of Table 1.7 presents results obtained by implementing this double reweighting approach. ${ }^{5}$

The item nonresponse function is modeled using the MAR assumption described in Section 5.3, while the AN assumption is maintained for the attrition function. ${ }^{6}$ We observe that our empirical results are robust to item nonresponse correction, since the $\mathcal{M}_{A N, N R}^{2, G M M}$ estimates still show non significant impact of the use of CTC on cash usage, both in terms of volume and value, at usual confidence levels. The impact of SVC is significant on cash in volume only, with an estimated effect around 2 percent.

These estimates highlight the need for central banks to act prudently in supplying cash to the public for transactional purposes. Retail payment innovations such as SVC are only affecting volume and not value. Recent work by Henry et al. (2015) using rich, detailed payment-diary data from the 2013 Bank of Canada Method-of-

\footnotetext{
${ }^{5}$ These estimates have been obtained using a parametric GMM approach instead of the nonparametric sieve approach, because the analytical variance expression of the non-parametric sieve double reweighting method is too involved. More results and robustness checks are available in Technical Appendix.

${ }^{6} \mathrm{Si}$ et al. (2015) work under similar assumptions in an imputation framework. In our empirical analysis, we assume that item nonresponse is MAR so as to generate comparable results with the imputation approach implemented as an alternative method to handle item nonresponse. The effect of attrition correction on the panel estimates observed on imputed samples is of the same order of magnitude than what is obtained on the incomplete data set (with item nonresponse). These results are available in Technical Appendix.
} 
Payments survey finds that the median transaction value for SVC is about $\$ 8$ in comparison to cash which is $\$ 9$. This finding validates our estimates in that SVC is used mostly for small transactions. In planning for the banknote production, if there is a need to reduce cash, central banks should concentrate on reducing banknotes amongst change notes $(\$ 5$ and $\$ 10)$, and possibly transactional notes $(\$ 20)$.

\subsubsection{Time-varying effects of innovations}

In order to capture the rapidly changing retail payment landscape for the years 2010 to 2012, we also obtain estimates on the 2010-11 and 2011-12 two-year panels, which allows for time-varying $\beta_{t}$. The results are reported in Table 1.8. The estimated impacts of $\mathrm{CTC}$ on the cash ratio in value and SVC on the cash ratio in volume are not significant in the first panel, but become significant in the second. A possible interpretation is that in 2010-11, CTC were not yet widely accepted by merchants. However, the turning point seems to occur around the 2011-12 panel, when almost all credit cards had a contactless functionality and acceptance was more widespread across merchants. As for SVC, this result might be related to the inflection point apparent in Figure 1.2 that reflects extensive use of SVC issued by specific merchants in recent years, as documented in Fung et al. (2015).

\subsection{Conclusions}

Gauging the impact of retail payment innovations on cash usage is an important public policy question. Central banks as the sole issuer of cash must understand the potential substitution from cash to retail payment innovations in order to plan for the design, production and distribution of cash. Market infrastructure participants

such as card processors and merchants likewise need to understand whether there is a demand for enhanced terminals. 
In this study, we utilize panel data to estimate the impact of these payment innovations on cash usage. However, econometric issues arise from non-ignorable attrition. We take advantage of the availability of refreshment samples to account for attrition without relying on rather restrictive assumptions. We find that accounting for unobserved heterogeneity and non-ignorable attrition is crucial for correctly assessing the impact of retail payment innovations on the relative cash usage of Canadian households. Except for the impact of SVC on cash in volume, we estimate impacts that are either non-significant or small, which differs from the findings of previous studies based on cross-sectional data. The use of CTC requires both that consumers have the card and merchants have the necessary physical infrastructure, whereas SVC is a specialized product that consumers know exactly where to use (e.g., coffee shops) for convenience. It is therefore not surprising that SVC lead to a reduction in cash usage.

There are several caveats to this study. First, payment innovations such as contactless credit cards are still in the nascent stage, so these estimates should be taken with caution. Second, we focus only on consumer outcomes and are silent on merchants' adoption of contactless terminals, due to the lack of micro level information. Camera et al. (2016) obtain experimental evidence in the laboratory and find little evidence of network externalities for retail payment innovations. It would be interesting to combine merchant information to directly estimate a theoretical two-sided markets model that would account for network externalities of retail payment innovations for both consumers and merchants. Disentangling network effects versus unobservable preferences of consumers and merchants is important for policymakers to understand the potential role of coordination in the payments market. Third, we consider the impacts only on cash. Future research will examine simultaneous implications for the use of credit cards and debit cards. 


\section{References}

Ai, C. And X. Chen (2003): "Efficient Estimation of Models with Conditional Moment Restrictions Containing Unknown Functions," Econometrica, 71, 17951843.

(2007): "Estimation of possibly misspecified semiparametric conditional moment restriction models with different conditioning variables," Journal of Econometrics, 141, 5-43.

Alvarez, F. AND F. Lippi (2009): "Financial Innovation and the Transactions Demand for Cash," Econometrica, 77, 363-402.

(2013): "The demand of liquid assets with uncertain lumpy expenditures," Journal of Monetary Economics, 60, 753-770.

(2015): "Cash burns - An inventory model with a cash-credit choice," EIEF Working Papers Series 1502, Einaudi Institute for Economics and Finance (EIEF).

Amromin, G. And S. Chakravorti (2009): "Whither Loose Change? The Diminishing Demand for Small-Denomination Currency," Journal of Money, Credit and Banking, 41, 315-335.

Arango, C., K. Huynh, B. Fung, And G. Stuber (2012): "The Changing Landscape for Retail Payments in Canada and the Implications for the Demand for Cash," Bank of Canada Review, 2012, 31-40.

Arango, C., K. P. Huynh, and L. Sabetti (2015): "Consumer payment choice: Merchant card acceptance versus pricing incentives," Journal of Banking and Finance, $55,130-141$.

Attanasio, O. R., L. Guiso, And T. Jappelli (2002): "The Demand for Money, Financial Innovation, and the Welfare Cost of Inflation: An Analysis with Household Data," Journal of Political Economy, 110, 317-351.

Bagnall, J., D. Bounie, K. P. Huynh, A. Kosse, T. Schmidt, S. Schuh, And H. Stix (2014): "Consumer Cash Usage: A Cross-Country Comparison with Payment Diary Survey Data," Staff Working Paper 2014-20, Bank of Canada.

Bhattacharya, D. (2006): "Inference in panel data models under attrition caused by unobservables," Working Paper.

(2008): "Inference in panel data models under attrition caused by unobservables," Journal of Econometrics, 144, 430-446.

Camera, G., M. Casari, and S. Bortolotti (2016): "An Experiment on Retail Payments Systems," Journal of Money, Credit and Banking, 48, 363-392. 
Chen, H., M.-H. Felt, And K. P. Huynh (2014): "Retail payment innovations and cash usage: Accounting for attrition using refreshment samples," Staff Working Paper 2014-27, Bank of Canada.

Cheng, T. C. And P. K. Trivedi (2015): "Attrition Bias in Panel Data: A Sheep in Wolf's Clothing? A Case Study Based on the Mabel Survey," Health Economics.

Committee on Payment and Settlement Systems, B. (2012): "Innovations in Retail Payments. Report of the Working Group on Innovations in Retail Payments," Tech. rep., Bank for International Settlements, http://www.bis.org/publ/cpss102.htm.

DAS, M. (2004): "Simple estimators for nonparametric panel data models with sample attrition," Journal of Econometrics, 120, 159-180.

Davezies, L. And X. DHaultf ceullle (2011): "Endogenous Attrition in Panels," Tech. rep.

Deng, Y., D. S. Hillygus, J. P. Reiter, Y. Si, and S. Zheng (2013): "Handling attrition in longitudinal studies: The case for refreshment samples," Statistical Science, 28, 238-256.

Fitzgerald, J., P. Gottschalk, and R. Moffitt (1998): "An Analysis of Sample Attrition in Panel Data: The Michigan Panel Study of Income Dynamics," Journal of Human Resources, 33, 251-299.

Fung, B., K. Huynh, And L. Sabetti (2014a): "The Impact of Retail Payment Innovations on Cash Usage," Journal of Financial Market Infrastructures, 3, 1-29.

Fung, B., K. Huynh, And G. Stuber (2015): "The Use of Cash in Canada," Bank of Canada Review, 2015, 45-56.

Fung, B., M. Molico, And G. Stuber (2014b): "Electronic Money and Payments: Recent Developments and Issues," Staff Discussion Paper 2014-2, Bank of Canada.

Gowrisankaran, G. and J. Stavins (2004): "Network Externalities and Technology Adoption: Lessons from Electronic Payments," RAND Journal of Economics, $35,260-276$.

Hausman, J. A. And D. A. Wise (1979): "Attrition bias in experimental and panel data: the Gary income maintenance experiment," Econometrica, 47, 455-473.

Henry, C., K. Huynh, And R. Shen (2015): "2013 Methods-of-Payment Survey Results," Staff Discussion Paper 2015-4, Bank of Canada.

Hirano, K., G. W. Imbens, G. Ridder, and D. B. Rubin (2001): "Combining panel data sets with attrition and refreshment samples," Econometrica, 69, 16451659. 
Horowitz, J. L. And E. Mammen (2004): "Nonparametric estimation of an additive model with a link function," The Annals of Statistics, 32, 2412-2443.

Huynh, K. P., P. Schmidt-Dengler, And H. Stix (2014): "The Role of Card Acceptance in the Transaction Demand for Money," CEPR Discussion Papers 10183, C.E.P.R. Discussion Papers.

KIM, S. (2012): "Sample Attrition in the Presence of Population Attrition," Working Papers UWEC-2009-02, University of Washington, Department of Economics.

Little, R. J. And D. B. Rubin (1987): Statistical analysis with missing data, vol. 539, Wiley New York.

Nevo, A. (2002): "Sample selection and information-theoretic alternatives to GMM," Journal of econometrics, 107, 149-157.

(2003): "Using weights to adjust for sample selection when auxiliary information is available," Journal of Business \& Economic Statistics, 21.

Nicoletti, C. (2006): "Nonresponse in dynamic panel data models," Journal of Econometrics, 132, 461-489.

Nijman, T. And M. Verbeek (1992): "Nonresponse in panel data: The impact on estimates of a life cycle consumption function," Journal of Applied Econometrics, $7,243-257$.

Rogoff, K. (2014): "Costs and Benefits to Phasing Out Paper Currency," in NBER Macroeconomics Annual 2014, Volume 29, National Bureau of Economic Research, Inc, NBER Chapters.

Rubin, D. B. (1976): "Inference and missing data," Biometrika, 63, 581-592.

Rysman, M. (2007): "An Empirical Analysis of Payment Card Usage," Journal of Industrial Economics, 55, 1-36.

Schmiedel, H., G. Kostova, And W. Ruttenberg (2012): "The Social and Private Costs of Retail Payment Instruments: A European Perspective," $E C B$ Occasional Paper.

Si, Y., J. P. Reiter, And D. S. Hillygus (2015): "Semi-parametric selection models for potentially non-ignorable attrition in panel studies with refreshment samples," Political Analysis, 23, 92-112.

Stango, V. (2000): "Competition and Pricing in the Credit Card Market," Review of Economics and Statistics, 82, 499-508.

Vella, F. AND M. Verbeek (1999): "Two-step estimation of panel data models with censored endogenous variables and selection bias," Journal of Econometrics, 90, 239-263. 
Wakamori, N. And A. Welte (2012): "Why Do Shoppers Use Cash? Evidence from Shopping Diary Data," Staff Working Paper No. 2012-24, Bank of Canada.

WoOdFord, M. (2003): Interest and prices: foundations of a theory of monetary policy, Princeton University Press.

WoOlDRIDGE, J. M. (2002): "Inverse probability weighted M-estimators for sample selection, attrition, and stratification," Portuguese Economic Journal, 1, 117-139. (2010): Econometric analysis of cross section and panel data, MIT press. 
TABLE 1.1: Demographic characteristics of CTC users and non-users

\begin{tabular}{|c|c|c|c|c|c|c|}
\hline & \multicolumn{2}{|c|}{2010} & \multicolumn{2}{|c|}{2011} & \multicolumn{2}{|c|}{2012} \\
\hline & $\mathrm{U}$ & $\mathrm{N}-\mathrm{U}$ & $\mathrm{U}$ & $\mathrm{N}-\mathrm{U}$ & $\mathrm{U}$ & $\mathrm{N}-\mathrm{U}$ \\
\hline City size: $<10 \mathrm{~K}$ & 14 & 19 & 15 & 19 & 12 & 19 \\
\hline $10-100 \mathrm{~K}$ & 13 & 14 & 12 & 14 & 13 & 14 \\
\hline $100 \mathrm{~K}-1 \mathrm{M}$ & 24 & 25 & 25 & 25 & 26 & 25 \\
\hline $1 \mathrm{M}+$ & 49 & 42 & 48 & 42 & 49 & 42 \\
\hline Household size: 1 & 22 & 28 & 23 & 28 & 23 & 28 \\
\hline 2 & 33 & 34 & 34 & 33 & 33 & 33 \\
\hline $3+$ & 45 & 39 & 43 & 39 & 44 & 39 \\
\hline Age of Head: $18-34$ & 23 & 19 & 24 & 19 & 22 & 19 \\
\hline $35-49$ & 34 & 30 & 32 & 30 & 33 & 29 \\
\hline $50-64$ & 25 & 28 & 27 & 28 & 26 & 30 \\
\hline $65+$ & 18 & 23 & 17 & 23 & 19 & 23 \\
\hline Income: $<25 \mathrm{~K}$ & 9 & 19 & 11 & 19 & 9 & 20 \\
\hline $25-44 \mathrm{~K}$ & 17 & 21 & 17 & 21 & 18 & 21 \\
\hline $45-69 \mathrm{~K}$ & 23 & 21 & 22 & 20 & 21 & 21 \\
\hline $70+\mathrm{K}$ & 51 & 39 & 51 & 39 & 53 & 39 \\
\hline Home Ownership & 76 & 67 & 76 & 67 & 76 & 67 \\
\hline Rent & 24 & 33 & 24 & 33 & 24 & 33 \\
\hline Unemployed & 34 & 41 & 36 & 41 & 35 & 41 \\
\hline Employed & 66 & 59 & 64 & 59 & 65 & 59 \\
\hline Province: $\mathrm{BC}$ & 9 & 14 & 11 & 14 & 11 & 14 \\
\hline $\mathrm{AB} / \mathrm{MB} / \mathrm{SK}$ & 15 & 17 & 14 & 18 & 14 & 17 \\
\hline $\mathrm{ON}$ & 43 & 36 & 43 & 35 & 44 & 35 \\
\hline $\mathrm{QC}$ & 26 & 25 & 25 & 26 & 24 & 26 \\
\hline NB/NF/NS/PEI & 8 & 8 & 7 & 8 & 6 & 8 \\
\hline Observations & 1,597 & 9,748 & 2,149 & 9,770 & 1,996 & 8,728 \\
\hline
\end{tabular}

Notes: $\mathrm{U}$ and $\mathrm{N}-\mathrm{U}$ denote users and non-users of the payment innovation. Numbers are in proportions. Sample weights are used in these computations. 
TABLE 1.2: Demographic characteristics of SVC users and non-users

\begin{tabular}{lcccccc} 
& \multicolumn{2}{c}{2010} & \multicolumn{2}{c}{2011} & \multicolumn{2}{c}{2012} \\
& $\mathrm{U}$ & $\mathrm{N}-\mathrm{U}$ & $\mathrm{U}$ & $\mathrm{N}-\mathrm{U}$ & $\mathrm{U}$ & $\mathrm{N}-\mathrm{U}$ \\
\hline City size: $<10 \mathrm{~K}$ & 14 & 20 & 13 & 21 & 15 & 19 \\
10-100K & 14 & 14 & 14 & 14 & 13 & 14 \\
100K-1M & 27 & 23 & 27 & 23 & 26 & 24 \\
1M+ & 45 & 43 & 46 & 42 & 46 & 42 \\
\hline Household size: 1 & 21 & 30 & 20 & 31 & 21 & 30 \\
2 & 32 & 35 & 32 & 35 & 33 & 34 \\
3+ & 47 & 36 & 48 & 34 & 46 & 36 \\
\hline Age of Head: 18-34 & 23 & 18 & 23 & 18 & 22 & 18 \\
35-49 & 34 & 28 & 35 & 27 & 34 & 27 \\
50-64 & 27 & 28 & 26 & 29 & 28 & 30 \\
65+ & 16 & 26 & 15 & 26 & 17 & 25 \\
\hline Income: $<25 K$ & 13 & 20 & 12 & 21 & 13 & 20 \\
25-44K & 18 & 22 & 17 & 22 & 18 & 22 \\
45-69K & 22 & 21 & 21 & 21 & 21 & 21 \\
70+K & 47 & 36 & 50 & 36 & 49 & 37 \\
\hline Home Ownership & 72 & 67 & 73 & 66 & 74 & 66 \\
Rent & 28 & 33 & 27 & 34 & 26 & 34 \\
\hline Unemployed & 33 & 44 & 33 & 44 & 33 & 45 \\
Employed & 67 & 56 & 67 & 56 & 67 & 55 \\
\hline Province: BC & 14 & 13 & 15 & 12 & 14 & 13 \\
AB/MB/SK & 18 & 16 & 19 & 15 & 19 & 15 \\
ON & 44 & 32 & 42 & 33 & 42 & 34 \\
QC & 16 & 31 & 17 & 31 & 17 & 31 \\
NB/NF/NS/PEI & 8 & 7 & 7 & 8 & 8 & 7 \\
\hline Observations & 4,059 & 6,950 & 4,510 & 7,125 & 3,911 & 6,562 \\
\hline \hline
\end{tabular}

Notes: $\mathrm{U}$ and N-U denote users and non-users of the payment innovation. Numbers are in proportions. Sample weights are used in these computations. 
TABLE 1.3: Usage patterns of payment innovations in two consecutive years

\begin{tabular}{ccccccc}
\multirow{2}{*}{$\mathbf{t} ; \mathbf{t}+\mathbf{1}$} & \multicolumn{6}{c}{$\mathbf{S V C}$} \\
\cline { 2 - 7 } & N-U;N-U & N-U;U & U;N-U & U;U & Total \\
\hline \multirow{4}{*}{ CTC } & N-U;N-U & 37 & 13 & 12 & 12 & 74 \\
& N-U;U & 4 & 1 & 1 & 2 & 8 \\
& U;N-U & 3 & 1 & 1 & 1 & 5 \\
& U;U & 5 & 2 & 2 & 3 & 13 \\
\cline { 2 - 7 } & Total & 49 & 17 & 16 & 18 & 100 \\
\hline \hline
\end{tabular}

Notes: $\mathrm{U}$ and $\mathrm{N}-\mathrm{U}$ denote users and non-users of the payment innovation. $t=2010,2011$. Numbers are in proportions, obtained from the 2010-2012 three-year balanced panel. 
TABLE 1.4: Representative samples, stayers and refreshers in the three-period panel

\begin{tabular}{lcc} 
Samples & Part. patterns & Counts \\
\hline \multicolumn{3}{l}{ Yearly representative samples: } \\
2010 & $1 .$. & 11,695 \\
2011 & .1. & 12,241 \\
2012 &. .1 & 11,023 \\
\hline Stayers: & & \\
$S_{12}=1$ & 11. & 5,699 \\
$S_{23}=1$ & .11 & 6,079 \\
$S_{13}=1$ & 1.1 & 4,859 \\
$S_{12} S_{23}=1$ & 111 & 3,853 \\
\hline Top-up refreshment samples: \\
2011 & 01. & 6,542 \\
2012 & .01 & 4,944 \\
\hline \multicolumn{1}{c}{ Total } & $\ldots$ & 22,175 \\
\hline \hline
\end{tabular}

Notes: $S_{t-1, t}$ is a dummy that indicates whether a household observed in year $t-1$ participates again in year $t$. Participation patterns indicate participation in 2010, 2011 and 2012: 1 means participation, 0 means no participation and [.] can be 0 or 1 . A household can receive and answer only one CFM questionnaire in a 12-month period. Counts give the number of households. 
TABLE 1.5: First-year demographic characteristics: attritors vs. stayers

\begin{tabular}{lcccc} 
& Stayers & Attritors & F-statistic & P-value \\
\hline City size: $<10 \mathrm{~K}$ & 0.197 & 0.171 & 7.0 & 0.008 \\
10-100K & 0.144 & 0.137 & 0.7 & 0.409 \\
100K-1M & 0.255 & 0.245 & 1.1 & 0.296 \\
1M+ & 0.404 & 0.446 & 12.4 & 0.000 \\
\hline Household size: 1 & 0.327 & 0.242 & 66.8 & 0.000 \\
2 & 0.373 & 0.321 & 24.1 & 0.000 \\
$3+$ & 0.300 & 0.437 & 125.0 & 0.000 \\
\hline Age of Head: 18-34 & 0.107 & 0.237 & 178.3 & 0.000 \\
$35-49$ & 0.225 & 0.338 & 110.2 & 0.000 \\
$50-64$ & 0.324 & 0.255 & 45.3 & 0.000 \\
65+ & 0.344 & 0.169 & 292.0 & 0.000 \\
\hline Income: $<25 \mathrm{~K}$ & 0.205 & 0.166 & 17.1 & 0.000 \\
25-44K & 0.217 & 0.202 & 2.6 & 0.108 \\
45-69K & 0.212 & 0.212 & 0.0 & 0.997 \\
$70+\mathrm{K}$ & 0.366 & 0.420 & 20.0 & 0.000 \\
\hline Home Ownership & 0.742 & 0.662 & 51.3 & 0.000 \\
Rent & 0.258 & 0.338 & 51.3 & 0.000 \\
\hline Unemployed & 0.483 & 0.365 & 105.0 & 0.000 \\
Employed & 0.517 & 0.635 & 105.0 & 0.000 \\
\hline Province: BC & 0.136 & 0.131 & 0.5 & 0.496 \\
AB/MB/SK & 0.182 & 0.161 & 5.2 & 0.022 \\
ON & 0.348 & 0.375 & 6.1 & 0.013 \\
QC & 0.242 & 0.261 & 3.3 & 0.071 \\
NB/NF/NS/PEI & 0.092 & 0.072 & 8.6 & 0.003 \\
\hline Observations & 3,853 & 7,842 & & \\
\hline \hline
\end{tabular}

Notes: Numbers are in proportions. Characteristics are measured in 2010. Stayers participate three years in a row. Attritors participate in 2010 but not in all three years. Sample weights are used in these computations. F-statistics test the equality of proportions between stayers and attritors. 
TABLE 1.6: First-year banking and payment characteristics: attritors vs. stayers

\begin{tabular}{lcccc} 
& Stayers & Attritors & F-statistic & P-value \\
\hline Cash ratio:Value & 0.209 & 0.221 & 1.3 & 0.255 \\
Volume & 0.360 & 0.362 & 0.0 & 0.849 \\
\hline CC balance & 2,139 & 3,390 & 8.7 & 0.003 \\
Bank accout balance & 16,547 & 10,457 & 14.8 & 0.000 \\
CC revolver (proportion) & 0.267 & 0.389 & 33.4 & 0.000 \\
Number of CC & 2.03 & 1.88 & 3.7 & 0.056 \\
Number of bank accounts & 2.25 & 2.26 & 0.0 & 0.896 \\
Number of DC & 2.10 & 2.36 & 11.1 & 0.001 \\
\hline Households that paid with (proportions): & 0.897 & 0.916 & 2.1 & 0.145 \\
Cash past week & 0.825 & 0.775 & 8.1 & 0.005 \\
CC past month & 0.665 & 0.832 & 70.1 & 0.000 \\
DC past month & 0.155 & 0.130 & 2.2 & 0.137 \\
CTC past month & 0.348 & 0.401 & 5.6 & 0.018 \\
SVC past month & 0.592 & 0.558 & 2.4 & 0.123 \\
Cheque past month & \multicolumn{3}{c}{} & \\
\hline Relative Expenditure Share: & 0.934 & 1.067 & 15.2 & 0.000 \\
Groceries & 0.950 & 1.043 & 2.5 & 0.116 \\
Food at restaurants/takeout & 0.829 & 1.103 & 9.8 & 0.002 \\
Food from convenience stores & 0.798 & 1.066 & 7.9 & 0.005 \\
Recreation & 0.890 & 1.025 & 5.4 & 0.020 \\
Automobile/gas & 889 & 3,272 & & \\
\hline Observations & & & & \\
\hline \hline
\end{tabular}

Notes: CC: credit card, DC: debit card. Balances are in dollars. Relative expenditure shares are ratios relative to the average within the household head's demographic stratum. Characteristics are measured in 2010. Stayers participate three years in a row. Attritors participate in 2010 but not in all three years. Sample weights are used in these computations. F-statistics test the equality of proportions between stayers and attritors. 


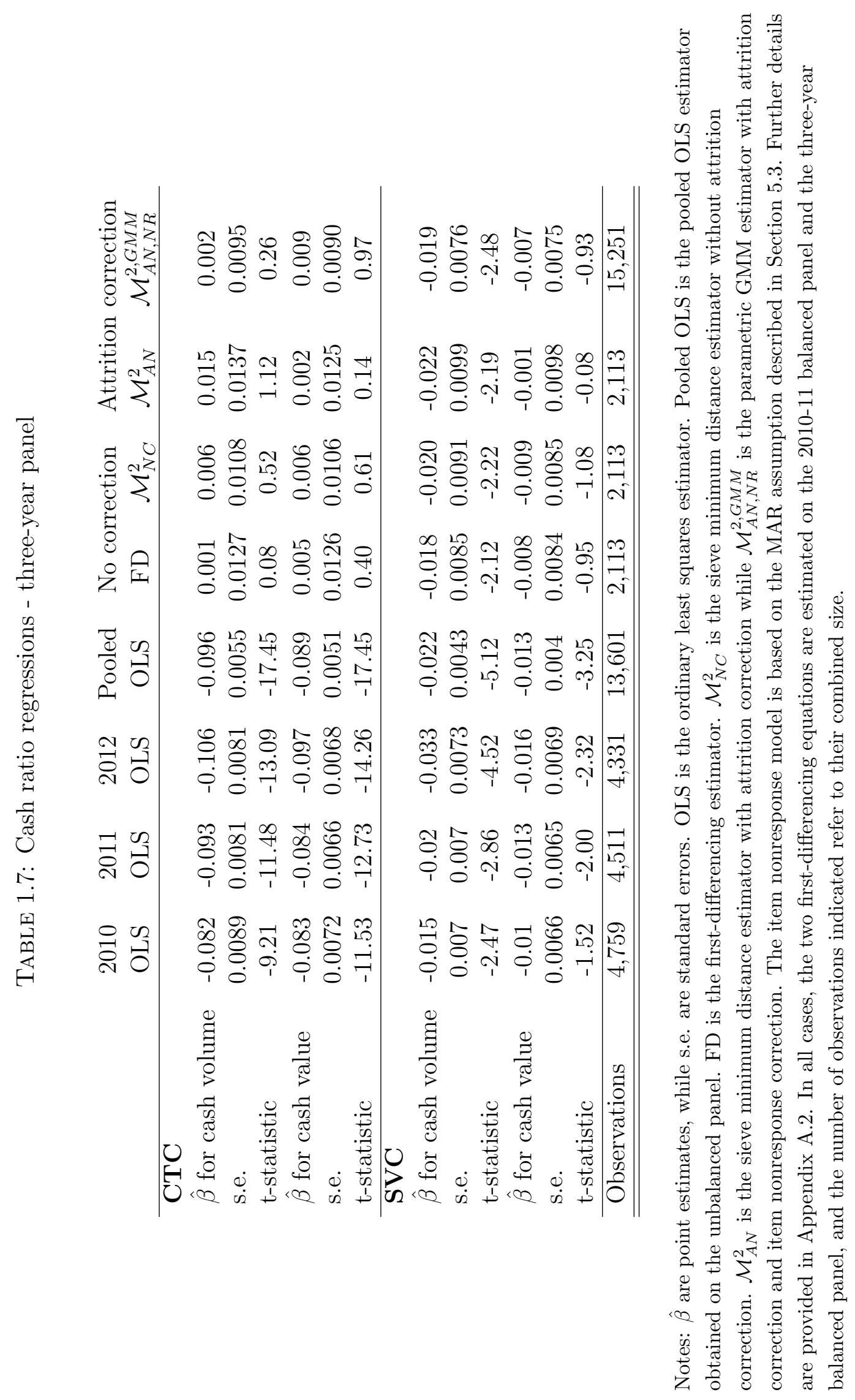


TABLE 1.8: Cash ratio regressions - two-year panels

\begin{tabular}{|c|c|c|c|c|c|c|}
\hline & \multirow{2}{*}{$\begin{array}{c}\text { Year } 1 \\
\text { OLS }\end{array}$} & \multirow{2}{*}{$\begin{array}{c}\text { Year } 2 \\
\text { OLS }\end{array}$} & \multirow{2}{*}{$\begin{array}{c}\text { Pooled } \\
\text { OLS }\end{array}$} & \multicolumn{2}{|c|}{ No correction } & \multirow{2}{*}{$\begin{array}{c}\text { Correction } \\
\mathcal{M}_{A N}^{1}\end{array}$} \\
\hline & & & & FD & $\mathcal{M}_{N C}^{1}$ & \\
\hline \multicolumn{7}{|l|}{ 2010-2011 panel } \\
\hline \multicolumn{7}{|l|}{ CTC } \\
\hline$\hat{\beta}$ for cash volume & -0.082 & -0.092 & -0.089 & -0.007 & -0.007 & -0.009 \\
\hline s.e. & 0.0085 & 0.0075 & 0.0063 & 0.0135 & 0.0111 & 0.0118 \\
\hline t-statistics & -9.65 & -12.27 & -14.13 & -0.52 & -0.61 & -0.79 \\
\hline$\hat{\beta}$ for cash value & -0.081 & -0.084 & -0.084 & 0.010 & 0.010 & 0.007 \\
\hline s.e. & 0.0068 & 0.0061 & 0.0059 & 0.0135 & 0.0100 & 0.0105 \\
\hline t-statistics & -11.91 & -13.77 & -14.24 & 0.74 & 1.04 & 0.66 \\
\hline \multicolumn{7}{|l|}{ SVC } \\
\hline$\hat{\beta}$ for cash volume & -0.014 & -0.021 & -0.018 & -0.014 & -0.013 & -0.006 \\
\hline s.e. & 0.0067 & 0.0064 & 0.0049 & 0.0094 & 0.0090 & 0.0102 \\
\hline t-statistics & -2.09 & -3.28 & -3.67 & -1.49 & -1.48 & -0.56 \\
\hline$\hat{\beta}$ for cash value & -0.009 & -0.015 & -0.013 & -0.008 & -0.008 & -0.004 \\
\hline s.e. & 0.0063 & 0.0059 & 0.0046 & 0.0093 & 0.0085 & 0.0095 \\
\hline t-statistics & -1.43 & -2.54 & -2.83 & -0.86 & -0.94 & -0.43 \\
\hline Observations & 5,158 & 5,360 & 10,518 & 3,500 & 3,500 & 3,500 \\
\hline Households & 5,158 & 5,360 & 8,768 & 1,750 & 1,750 & 1,750 \\
\hline \multicolumn{7}{|l|}{ 2011-2012 panel } \\
\hline \multicolumn{7}{|l|}{ CTC } \\
\hline$\hat{\beta}$ for cash volume & -0.094 & -0.103 & -0.098 & -0.007 & -0.008 & -0.010 \\
\hline s.e. & 0.0076 & 0.0079 & 0.0061 & 0.0125 & 0.0110 & 0.0118 \\
\hline t-statistics & -12.37 & -13.04 & -16.07 & -0.56 & -0.72 & -0.86 \\
\hline$\hat{\beta}$ for cash value & -0.085 & -0.097 & -0.091 & -0.020 & -0.021 & -0.027 \\
\hline s.e. & 0.0063 & 0.0065 & 0.0057 & 0.0126 & 0.0100 & 0.0110 \\
\hline t-statistics & -13.49 & -14.92 & -15.96 & -1.59 & -2.11 & -2.44 \\
\hline \multicolumn{7}{|l|}{ SVC } \\
\hline$\hat{\beta}$ for cash volume & -0.021 & -0.031 & -0.026 & -0.033 & -0.030 & -0.027 \\
\hline s.e. & 0.0065 & 0.0071 & 0.0050 & 0.0086 & 0.0083 & 0.0083 \\
\hline t-statistics & -3.23 & -4.37 & -5.20 & -3.84 & -3.57 & -3.27 \\
\hline$\hat{\beta}$ for cash value & -0.013 & -0.015 & -0.014 & -0.011 & -0.011 & -0.006 \\
\hline s.e. & 0.0061 & 0.0066 & 0.0047 & 0.0087 & 0.0078 & 0.0081 \\
\hline t-statistics & -2.13 & -2.27 & -2.98 & -1.26 & -1.39 & -0.72 \\
\hline Observations & 5,262 & 4,733 & 9,995 & 3,826 & 3,826 & 3,826 \\
\hline Households & 5,262 & 4,733 & 8,082 & 1,913 & 1,913 & 1,913 \\
\hline
\end{tabular}

Notes: $\hat{\beta}$ are point estimates, while s.e. are standard errors. OLS is the ordinary least squares estimator. Pooled OLS is the pooled OLS estimator obtained on the unbalanced panel. FD is the first-differencing estimator. $\mathcal{M}_{N C}^{1}$ and $\mathcal{M}_{A N}^{1}$ are sieve minimum distance estimators, without and with attrition correction. In all cases, the first-differencing equation is estimated on a two-year balanced panel, and the number of observations indicated refer to its size. Details are provided in Appendix A.2. 
Figure 1.1: Contactless card and terminal diffusion

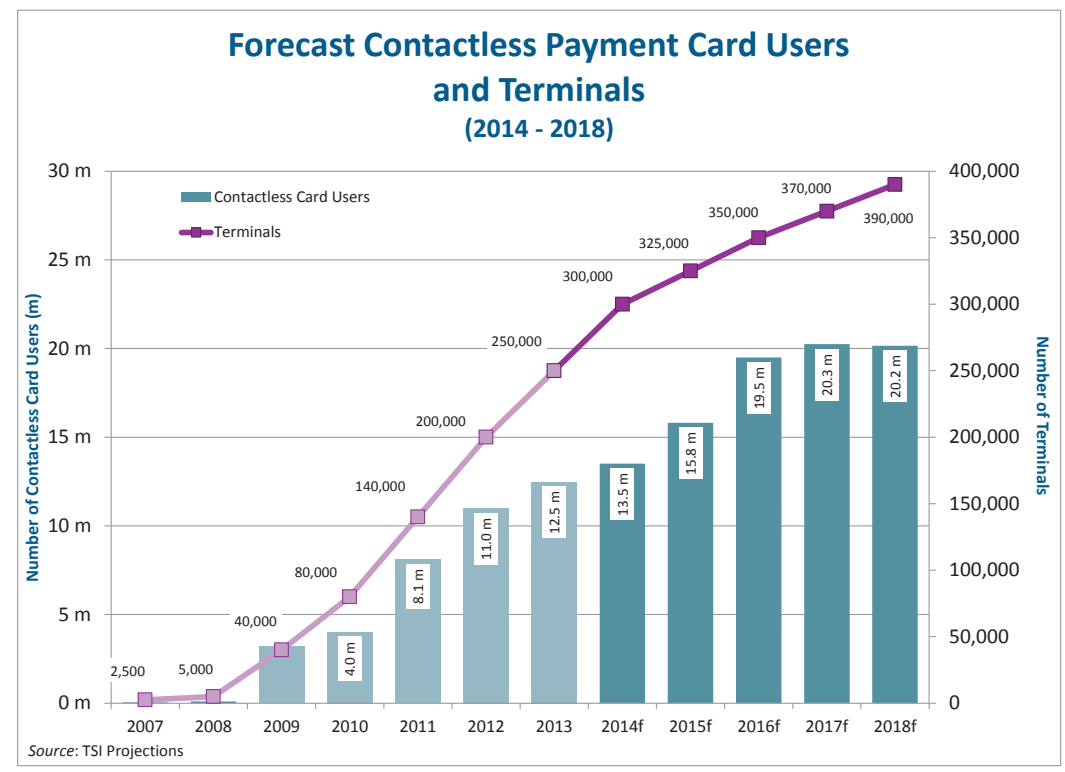

Source: 2014 Canadian Payments Forecast from Technology Strategies Inc. The left y-axis depicts the number of contactless card users in millions while the right y-axis is the number of POS terminals.

Figure 1.2: Stored-value cards in value and volume

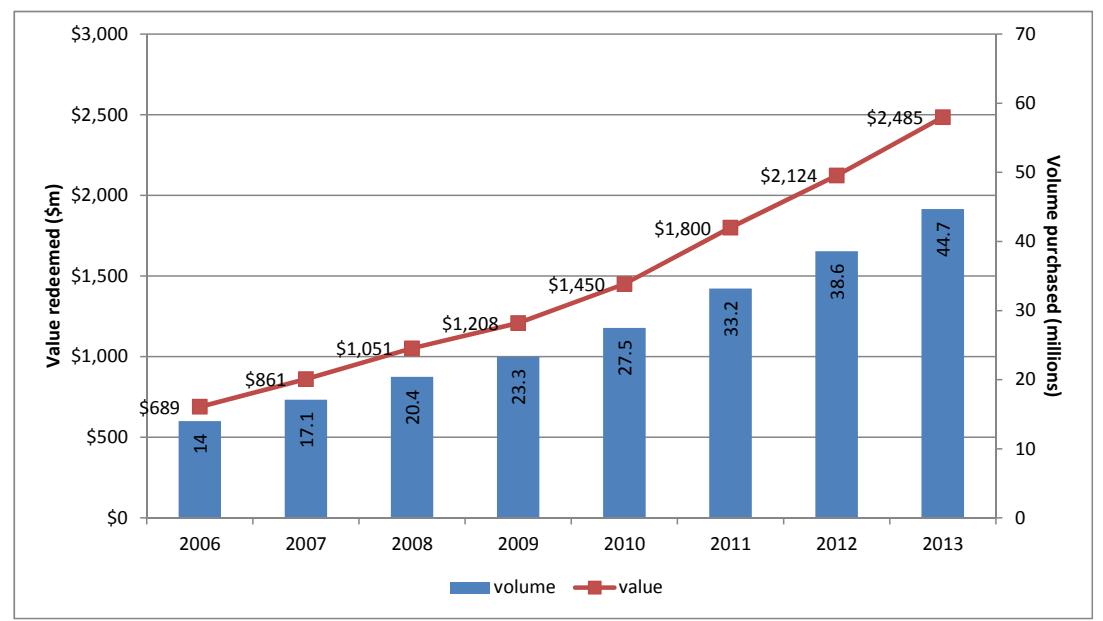

Source: 2014 Canadian Payments Forecast from Technology Strategies Inc. The left y-axis depicts the value redeemed in millions of dollars while the right $y$-axis is the volume of cards purchased in millions. 
Figure 1.3: Cash ratios of CTC and SVC users and non-users
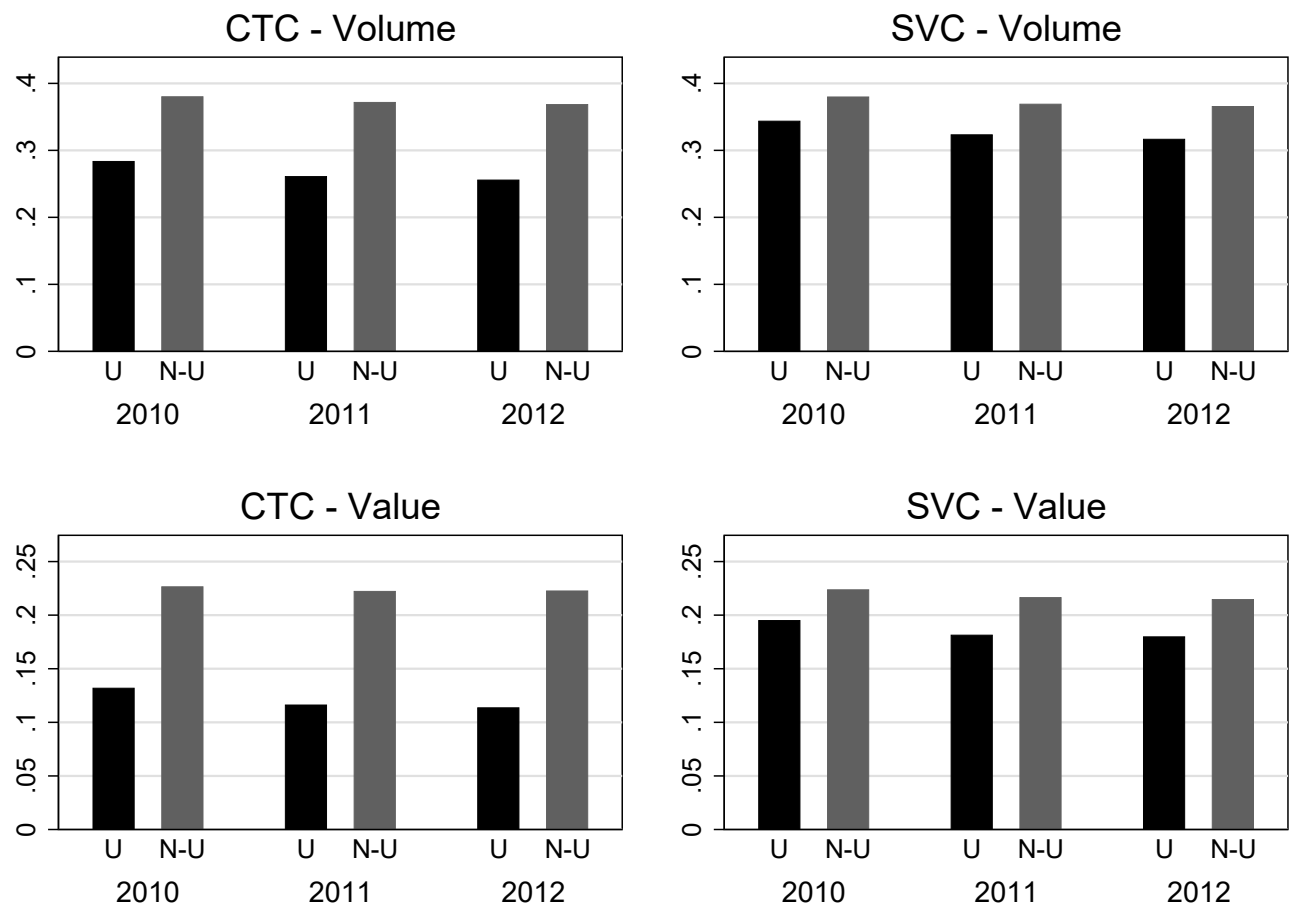

Notes: CTC and SVC denote contactless credit cards and single-purpose stored-value cards, respectively. $\mathrm{U}$ and $\mathrm{N}-\mathrm{U}$ denote users and non-users of the payment innovation. Numbers are in proportions. Sample weights are used in these computations. 
Figure 1.4: CTC, attrition probability versus the cash ratio in volume
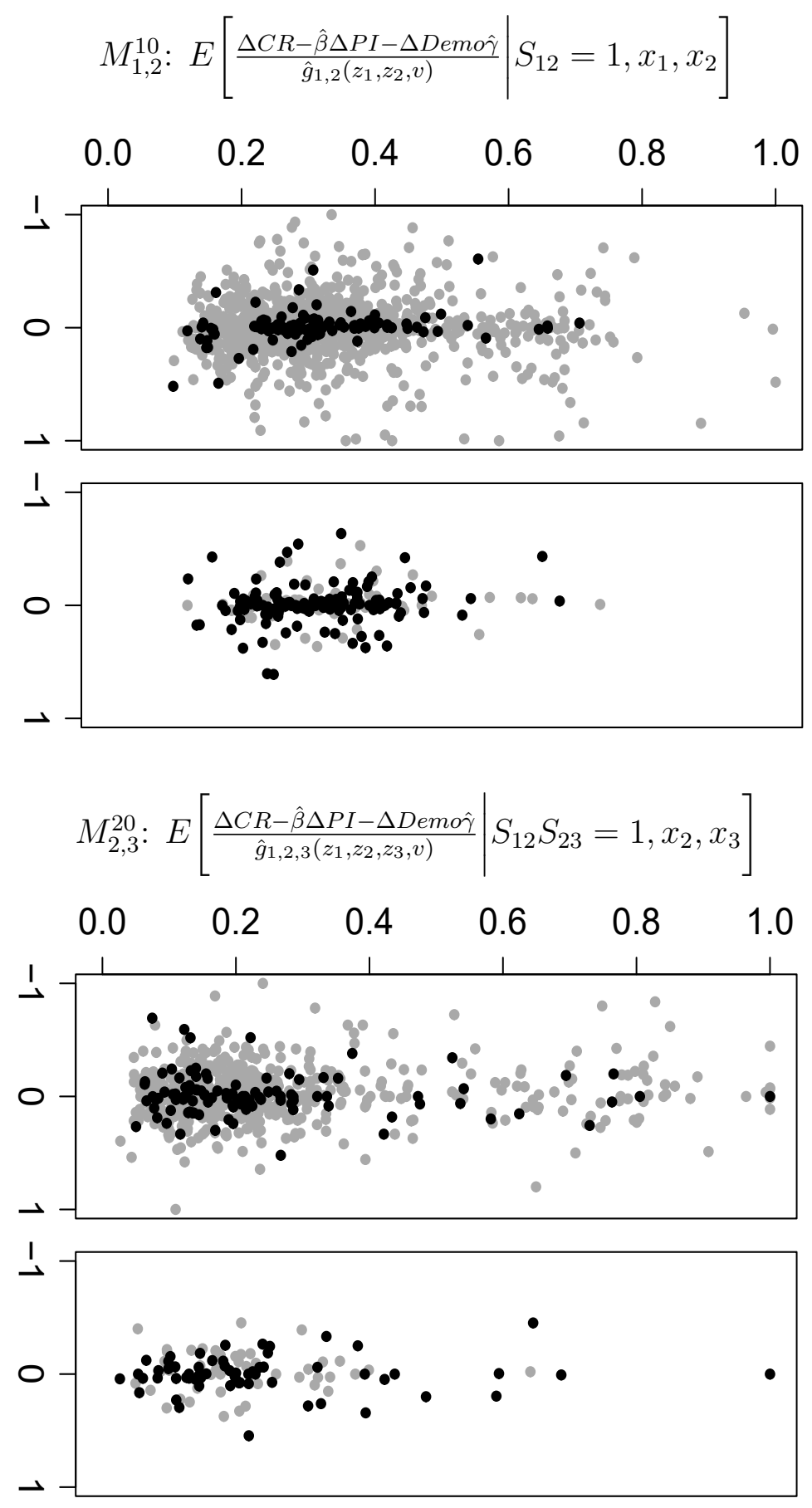

Notes: The estimated survival function, $\hat{g}(\cdot)$, is on the y-axis while the change in the cash ratio is on the x-axis. The functions $\hat{g}_{1,2}$ (from $M_{1,2}^{10}$ ) and $\hat{g}_{1,2,3}$ (from $M_{2,3}^{20}$ ) are depicted in the first two and last two panes, respectively. The first and third panes depict the never-users (N-U;N-U) in grey and the always-users $(\mathrm{U} ; \mathrm{U})$ in black; the second and fourth panes contain the stop-users $(\mathrm{U} ; \mathrm{N}-\mathrm{U})$ in grey and the new-users $(\mathrm{N}-\mathrm{U} ; \mathrm{U})$ in black. 
Figure 1.5: SVC, attrition probability versus the cash ratio in volume
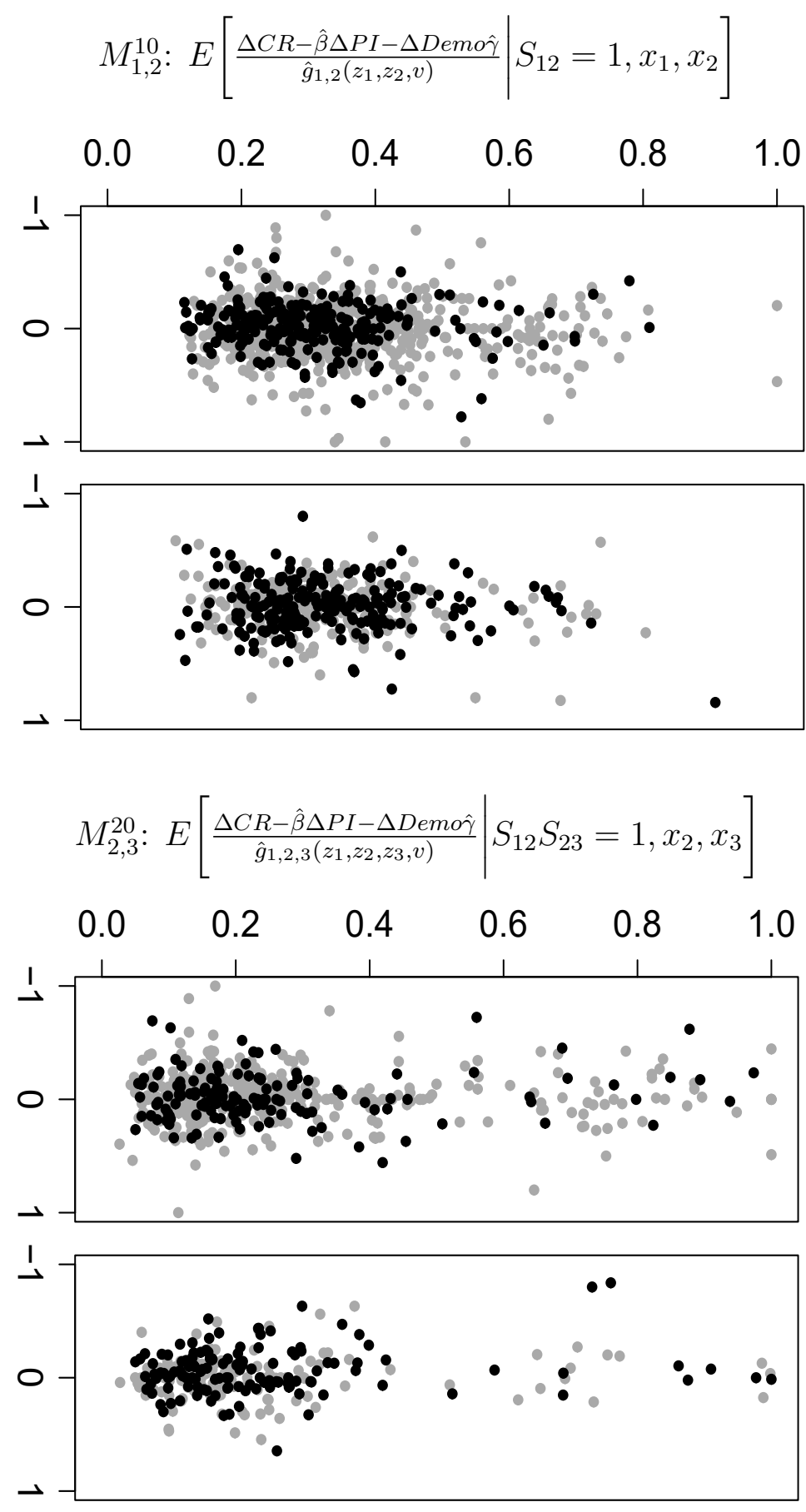

Notes: The estimated survival function, $\hat{g}(\cdot)$, is on the y-axis while the change in the cash ratio is on the x-axis. The functions $\hat{g}_{1,2}$ (from $M_{1,2}^{10}$ ) and $\hat{g}_{1,2,3}$ (from $M_{2,3}^{20}$ ) are depicted in the first two and last two panes, respectively. The first and third panes depict the never-users (N-U;N-U) in grey and the always-users $(\mathrm{U} ; \mathrm{U})$ in black; the second and fourth panes contain the stop-users $(\mathrm{U} ; \mathrm{N}-\mathrm{U})$ in grey and the new-users $(\mathrm{N}-\mathrm{U} ; \mathrm{U})$ in black. 


\section{Appendix A}

\section{Appendix to Chapter 1}

\section{A.1 Variables Description}

This section describes the variables from the Canadian Financial Monitor used in our analysis.

\section{Cash ratios:}

- Cash ratio in volume: ratio of the total number of purchases paid in cash in the past month to the total number of all purchases in the past month.

- Cash ratio in value: ratio of the total value of purchases paid in cash in the past month to the total value of all purchases in the past month.

2. Payment innovation variables indicating CTC or SVC users: Dummy variables indicating whether any member of the household used a given payment innovation to make purchases in the past month.

\section{Demographics:}

- Log of head age and log of head age squared: logarithm and square of logarithm of the household head's age.

- Income: household income for the past year before taxes, a categorical variable of which the base category is 'Under $25 \mathrm{~K}^{\prime}$. 
- Internet user: a dummy variable indicating whether any member of the household uses the internet.

- CC revolver: a dummy variable indicating whether any member of the household revolved on his/her credit card balance in the past month.

4. Types of expenditure: To avoid potential endogeneity issues, we measure household expenditures in various categories as a ratio relative to the average within the individual's demographic stratum (defined according to age and income group), following Stango (2000). Expenditure categories considered are: groceries, including beverages; food and beverages at restaurants/clubs/bars; snacks and beverages from convenience stores; recreation; automobile maintenance/gas. For each household, we calculate the share of expenditures made in each category in the past month relative to the total value of purchases made in the past month. 


\section{A.2 Attrition Functions and Moment Conditions}

TABLE A.1: Description of the estimated models

\begin{tabular}{|c|c|c|c|c|}
\hline Model & $\mathrm{EF}$ & SF & CRF & Moments \\
\hline $\mathcal{M}_{N C}^{1}$ & $\phi_{t-1, t}$ & 1 & & $M_{t-1, t}^{10}$ \\
\hline $\mathcal{M}_{A N}^{1}$ & $\phi_{t-1, t}$ & $g_{t-1, t}$ & & $M_{t-1, t}^{10}, M_{t-1}^{11}, M_{t}^{12}$ \\
\hline $\mathcal{M}_{N C}^{2}$ & $\left\{\begin{array}{l}\phi_{1,2} \\
\phi_{2,3}\end{array}\right.$ & $\left\{\begin{array}{l}1 \\
1\end{array}\right.$ & & $\left\{\begin{array}{l}M_{1,2}^{10} \\
M_{2,3}^{20}\end{array}\right.$ \\
\hline $\mathcal{M}_{A N}^{2}$ & $\left\{\begin{array}{l}\phi_{1,2} \\
\phi_{2,3}\end{array}\right.$ & $\left\{\begin{array}{l}g_{1,2} \\
g_{1,2,3}\end{array}\right.$ & & $\left\{\begin{array}{l}M_{1,2}^{10}, M_{1}^{11}, M_{2}^{12} \\
M_{2,3}^{20}, M_{1}^{21}, M_{2}^{22}, M_{3}^{23}\end{array}\right.$ \\
\hline $\mathcal{M}_{A N, N R}^{2}$ & $\left\{\begin{array}{l}\phi_{1,2} \\
\phi_{2,3}\end{array}\right.$ & $\left\{\begin{array}{l}g_{1,2}^{*} \\
g_{1,2,3}^{*}\end{array}\right.$ & $\begin{array}{l}h_{1} \\
h_{2} \\
h_{3}\end{array}$ & $\left\{\begin{array}{l}M_{1,2}^{10^{*}}, M_{1}^{11^{*}}, M_{2}^{12^{*}} \\
M_{2,3}^{20}, M_{1}^{21^{*}}, M_{2}^{22^{*}}, M_{3}^{23^{*}} \\
M_{1}^{h 1}, M_{2}^{h 2}, M_{3}^{h 3}\end{array}\right.$ \\
\hline
\end{tabular}

The estimation function (EF) is defined as

$\phi_{t-1, t}: \quad \phi\left(z_{t-1}, z_{t}, \beta\right)=\Delta C R_{t}=\beta \Delta P I_{t}+\Delta D e m o_{t} \gamma+\Delta u_{t}$ for $t=2,3$.

The survival functions (SF) under the AN assumption are defined as

$$
\begin{aligned}
& g_{t-1, t}: \operatorname{Pr}\left(S_{t-1, t}=1 \mid z_{t-1}, z_{t}, v\right) \equiv g\left(k_{0}(v)+k_{1}\left(z_{t-1}, v\right)+k_{2}\left(z_{t}, v\right)\right) \text { for } t=2,3, \\
& g_{1,2,3}: \operatorname{Pr}\left(S_{12} S_{23}=1 \mid z_{1}, z_{2}, z_{3}, v\right) \equiv g\left(k_{0}(v)+k_{1}\left(z_{1}, v\right)+k_{2}\left(z_{2}, v\right)+k_{3}\left(z_{3}, v\right)\right) .
\end{aligned}
$$

The moments used in models $\mathcal{M}_{N C}^{1}$ to $\mathcal{M}_{A N}^{2}$ are defined as

$$
\begin{array}{ll}
M_{t-1, t}^{10}: & m_{10}\left(x_{t-1}, x_{t} ; \delta\right) \equiv E\left[\frac{\phi\left(z_{t-1}, z_{t}, \beta\right)}{g_{t-1, t}\left(z_{t-1}, z_{t}, v\right)} \mid S_{t-1, t}=1, x_{t-1}, x_{t}\right]=0, \\
M_{t-1}^{11}: & m_{11}\left(z_{t-1}, v ; \delta\right) \equiv E\left[\frac{S_{t-1, t}}{g_{t-1, t}\left(z_{t-1}, z_{t}, v\right)}-1 \mid z_{t-1}, v\right]=0, \\
M_{t}^{12}: & m_{12}\left(z_{t}, v ; \delta\right) \equiv E\left[\frac{S_{t-1, t}}{g_{t-1, t}\left(z_{t-1}, z_{t}, v\right)}-1 \mid z_{t}, v\right]=0, \\
M_{2,3}^{20}: & m_{20}\left(x_{2}, x_{3} ; \delta\right) \equiv E\left[\frac{\phi\left(z_{2}, z_{3}, \beta\right)}{g_{1,2,3}\left(z_{1}, z_{2}, z_{3}, v\right)} \mid S_{12} S_{23}=1, x_{2}, x_{3}\right]=0, \\
M_{1}^{21}: & m_{21}\left(z_{1}, v ; \delta\right) \equiv E\left[\frac{S_{12} S_{23}}{g_{1,2,3}\left(z_{1}, z_{2}, z_{3}, v\right)}-1 \mid z_{1}, v\right]=0, \\
M_{2}^{22}: & m_{22}\left(z_{2}, v ; \delta\right) \equiv E\left[\frac{S_{12} S_{23}}{g_{1,2,3}\left(z_{1}, z_{2}, z_{3}, v\right)}-1 \mid z_{2}, v\right]=0, \\
M_{3}^{23}: & m_{23}\left(z_{3}, v ; \delta\right) \equiv E\left[\frac{S_{12} S_{23}}{g_{1,2,3}\left(z_{1}, z_{2}, z_{3}, v\right)}-1 \mid z_{3}, v\right]=0 .
\end{array}
$$


In the case where item nonresponse is also corrected for, the SF become

$$
\begin{aligned}
& g_{1,2}^{*}: \operatorname{Pr}\left(S_{12} C_{1} C_{2}=1 \mid z_{1}, z_{2}, v\right) \equiv g^{*}\left(k_{0}(v)+k_{1}\left(z_{1}, v\right)+k_{2}\left(z_{2}, v\right)\right), \\
& g_{1,2,3}^{*}: \operatorname{Pr}\left(S_{12} S_{23} C=1 \mid z_{1}, z_{2}, z_{3}, v\right) \equiv g^{*}\left(k_{0}(v)+k_{1}\left(z_{1}, v\right)+k_{2}\left(z_{2}, v\right)+k_{3}\left(z_{3}, v\right)\right),
\end{aligned}
$$

The item complete-response functions $(\mathrm{CRF})$ under the MAR assumption are defined as

$$
\begin{aligned}
& h_{1}: \operatorname{Pr}\left(C_{1}=1 \mid w_{1}, v\right) \equiv h\left(l_{1}\left(w_{1}, v\right)\right), \\
& h_{2}: \operatorname{Pr}\left(C_{2}=1 \mid w_{2}, v\right) \equiv h\left(l_{2}\left(w_{2}, v\right)\right), \\
& h_{3}: \operatorname{Pr}\left(C_{3}=1 \mid w_{3}, v\right) \equiv h\left(l_{3}\left(w_{3}, v\right)\right),
\end{aligned}
$$

The moments used in model $\mathcal{M}_{A N, N R}^{2}$ are as follows

$$
\begin{aligned}
& M_{1,2}^{10^{*}}: m_{10}^{*}\left(x_{1}, x_{2} ; \delta\right) \equiv E\left[\frac{\phi\left(z_{1}, z_{2}, \beta\right)}{g_{1,2}^{*}\left(z_{1}, z_{2}, v\right)} \mid S_{12}=1, C_{1} C_{2}=1, x_{1}, x_{2}\right]=0, \\
& M_{1}^{11^{*}}: m_{11}^{*}\left(z_{1}, v ; \delta\right) \equiv E\left[\left(\frac{S_{12} C_{1} C_{2}}{g_{1,2}^{*}\left(z_{1}, z_{2}, v\right)}-1\right) \frac{C_{1}}{h_{1}\left(w_{1}, v\right)} \mid z_{1}, v\right]=0, \\
& M_{2}^{12^{*}}: m_{12}^{*}\left(z_{2}, v ; \delta\right) \equiv E\left[\left(\frac{S_{12} C_{1} C_{2}}{g_{1,2}^{*}\left(z_{1}, z_{2}, v\right)}-1\right) \frac{C_{2}}{h_{2}\left(w_{2}, v\right)} \mid z_{2}, v\right]=0, \\
& M_{2,3}^{20^{*}}: m_{20}^{*}\left(x_{2}, x_{3} ; \delta\right) \equiv E\left[\frac{\phi\left(z_{2}, z_{3}, \beta\right)}{g_{1,2,3}^{*}\left(z_{1}, z_{2}, z_{3}, v\right)} \mid S_{12} S_{23}=1, C=1, x_{2}, x_{3}\right]=0 \text {, } \\
& M_{1}^{21^{*}}: m_{21}^{*}\left(z_{1}, v ; \delta\right) \equiv E\left[\left(\frac{S_{12} S_{23} C}{g_{1,2,3}^{*}\left(z_{1}, z_{2}, z_{3}, v\right)}-1\right) \frac{C_{1}}{h_{1}\left(w_{1}, v\right)} \mid z_{1}, v\right]=0, \\
& M_{2}^{22^{*}}: m_{22}^{*}\left(z_{2}, v ; \delta\right) \equiv E\left[\left(\frac{S_{12} S_{23} C}{g_{1,2,3}^{*}\left(z_{1}, z_{2}, z_{3}, v\right)}-1\right) \frac{C_{2}}{h_{2}\left(w_{2}, v\right)} \mid z_{2}, v\right]=0, \\
& M_{3}^{23^{*}}: m_{23}^{*}\left(z_{3}, v ; \delta\right) \equiv E\left[\left(\frac{S_{12} S_{23} C}{g_{1,2,3}^{*}\left(z_{1}, z_{2}, z_{3}, v\right)}-1\right) \frac{C_{3}}{h_{3}\left(w_{3}, v\right)} \mid z_{3}, v\right]=0, \\
& M_{1}^{h 1}: \quad m_{h 1}\left(w_{1}, v ; \delta\right) \equiv E\left[\frac{C_{1}}{h_{1}\left(w_{1}, v\right)}-1 \mid w_{1}, v\right]=0, \\
& M_{2}^{h 2}: \quad m_{h 2}\left(w_{2}, v ; \delta\right) \equiv E\left[\frac{C_{2}}{h_{2}\left(w_{2}, v\right)}-1 \mid w_{2}, v\right]=0 \text {, } \\
& M_{3}^{h 3}: \quad m_{h 3}\left(w_{3}, v ; \delta\right) \equiv E\left[\frac{C_{3}}{h_{3}\left(w_{3}, v\right)}-1 \mid w_{3}, v\right]=0 .
\end{aligned}
$$




\section{A.3 Proof of Proposition 1}

The proof essentially follows the Bhattacharya (2008) two-period panel case. Let $\kappa_{0}(\cdot)$ be the true function while $\kappa(\cdot)$ is a generic candidate function where

$$
\begin{aligned}
& \kappa_{0}=k_{00}(\cdot)+k_{10}(\cdot, \cdot)+k_{20}(\cdot, \cdot)+k_{30}(\cdot, \cdot), \\
& \kappa=k_{0}(\cdot)+k_{1}(\cdot, \cdot)+k_{2}(\cdot, \cdot)+k_{3}(\cdot, \cdot) .
\end{aligned}
$$

Below we suppress the arguments $\left(z_{1}, z_{2}, z_{3}, v\right)$ in $\kappa_{0}$ and $\kappa$. Notice that we have:

$$
\begin{aligned}
& E\left[\frac{g\left(\kappa_{0}\right)}{g(\kappa)}-1 \mid z_{1}, v\right]=0 \text { a.e. } z_{1}, v \\
& E\left[\frac{g\left(\kappa_{0}\right)}{g(\kappa)}-1 \mid z_{2}, v\right]=0 \text { a.e. } z_{2}, v \\
& E\left[\frac{g\left(\kappa_{0}\right)}{g(\kappa)}-1 \mid z_{3}, v\right]=0 \text { a.e. } z_{3}, v .
\end{aligned}
$$

This implies, by the law of iterated expectation, that for $w_{0}(v)=k_{00}(v)-k_{0}(v)$ and

$$
\begin{aligned}
& w_{1}\left(z_{1}, v\right)=k_{10}\left(z_{1}, v\right)-k_{1}\left(z_{1}, v\right), \\
& w_{2}\left(z_{2}, v\right)=k_{20}\left(z_{2}, v\right)-k_{2}\left(z_{2}, v\right), \\
& w_{3}\left(z_{3}, v\right)=k_{30}\left(z_{3}, v\right)-k_{3}\left(z_{3}, v\right),
\end{aligned}
$$




$$
\begin{aligned}
& E\left[\frac{g\left(\kappa_{0}\right)-g(\kappa)}{g(\kappa)}\left\{\kappa_{0}-\kappa\right\} \mid v\right] \\
& \quad=E\left[\frac{g\left(\kappa_{0}\right)-g(\kappa)}{g(\kappa)}\left\{w_{0}(v)+w_{1}\left(z_{1}, v\right)+w_{2}\left(z_{3}, v\right)+w_{3}\left(z_{3}, v\right)\right\} \mid v\right] \\
& \quad=E\left[\left\{w_{0}(v)+w_{1}\left(z_{1}, v\right)\right\} E\left[\frac{g\left(\kappa_{0}\right)-g(\kappa)}{g(\kappa)} \mid z_{1}, v\right] \mid v\right] \\
& \quad+E\left[w_{2}\left(z_{2}, v\right) E\left[\frac{g\left(\kappa_{0}\right)-g(\kappa)}{g(\kappa)} \mid z_{2}, v\right] \mid v\right] \\
& \quad+E\left[w_{3}\left(z_{3}, v\right) E\left[\frac{g\left(\kappa_{0}\right)-g(\kappa)}{g(\kappa)} \mid z_{3}, v\right] \mid v\right] \\
& \quad=0 .
\end{aligned}
$$

Since $g$ is strictly increasing, $\left[g\left(\kappa_{0}\right)-g(\kappa)\right] \times\left[\kappa_{0}-\kappa\right]$ is strictly positive w.p. 1 if $\kappa \neq \kappa_{0}$. This is because if $\kappa_{0}>\kappa$, then $g\left(\kappa_{0}\right)-g(\kappa)>0$ and if $\kappa>\kappa_{0}$, then $g\left(\kappa_{0}\right)-g(\kappa)<0$. In either case, $g\left(\kappa_{0}\right)-g(\kappa)$ has the same sign as $\left[\kappa_{0}-\kappa\right]$. Next, $g(\cdot)$ is a c.d.f. and therefore non-negative; so the random variable $\frac{g\left(\kappa_{0}\right)-g(\kappa)}{g(\kappa)}\left[\kappa_{0}-\kappa\right]$ is non-negative w.p.1. Then for the condition:

$$
\begin{aligned}
& E\left[\frac{g\left(\kappa_{0}\right)-g(\kappa)}{g(\kappa)}\left\{\kappa_{0}-\kappa\right\} \mid v\right] \\
& \quad=E\left[\frac{g\left(\kappa_{0}\right)-g(\kappa)}{g(\kappa)}\left\{w_{0}(v)+w_{1}\left(z_{1}, v\right)+w_{2}\left(z_{3}, v\right)+w_{3}\left(z_{3}, v\right)\right\} \mid v\right] \\
& \quad=0
\end{aligned}
$$

to hold, we must have: for each fixed $v$,

$$
w_{0}(v)+w_{1}\left(z_{1}, v\right)+w_{2}\left(z_{2}, v\right)+w_{3}\left(z_{3}, v\right)=0
$$

for all $z_{1}, z_{2}, z_{3} \in \mathcal{Z}_{1}(v) \times \mathcal{Z}_{2}(v) \times \mathcal{Z}_{3}(v)$. By (i), we must have w.p.1 for each $v$, $w_{1}\left(z_{1}, v\right)$ does not depend on $z_{1}, w_{2}\left(z_{2}, v\right)$ does not depend on $z_{2}, w_{3}\left(z_{3}, v\right)$ does not 
depend on $z_{3}$. Then by (iv), we have for each $v$,

$$
\begin{aligned}
& w_{1}\left(z_{1}, v\right)=w_{1}\left(\bar{z}_{1}(v), v\right)=0 \text { for all } z_{1} \\
& w_{2}\left(z_{2}, v\right)=w_{2}\left(\bar{z}_{2}(v), v\right)=0 \text { for all } z_{2} \\
& w_{3}\left(z_{3}, v\right)=w_{3}\left(\bar{z}_{3}(v), v\right)=0 \text { for all } z_{3},
\end{aligned}
$$

which implies the above condition. 


\section{Appendix B}

\section{Technical Appendix to Chapter 1}

\section{B.1 Outline}

This technical appendix is organized as follows. Section B.2 describes identification and estimation for the two-period attrition function using a refreshment sample. Additional estimation results obtained on the two-year panels are also presented. In Section B.3, we discuss the practical implementation of the sieve minimum distance (SMD) estimator. Section B.4 provides empirical results on the joint impact of CTC and SVC, in order to allow for the possibility that a household adopts more than one type of innovation at the same time. In Section B.5 we discuss the incidence of item nonresponse in the data in more details and present supplemental estimation results. 


\section{B.2 Correcting for Attrition in a Two-period panel with Refreshment}

\section{B.2.1 Identification of a two-period attrition function with refreshment}

We set the two-period panel as follows. The primary sample of size $n_{1}$ is a random draw from the population in period 1. In period 2 , however, only $n_{12}\left(n_{12}<n_{1}\right)$ remain (i.e., participate again). This two-period panel is unbalanced because of attrition: both $z_{1}=\left(x_{1}, y_{1}\right)$ and $z_{2}=\left(x_{2}, y_{2}\right)$ are observed for stayers ( $n_{12}$ units), but for attritors $\left(n_{1}-n_{12}\right.$ units) we only observe $z_{1}$. In the refreshment sample, on the other hand, only $z_{2}$ is observed; see Figure B.1. ${ }^{1}$

Hirano et al. (2001) show that, under the additive non-ignorable (or quasi-separability) restriction, the attrition function $g\left(z_{1}, z_{2}\right) \equiv g\left(k_{1}\left(z_{1}\right)+k_{2}\left(z_{2}\right)\right)$ is identified by the following two integral equations, where $g$ is known and functions $k_{1}$ and $k_{2}$ are nonparametrically identified up to a location normalization:

$$
\begin{aligned}
& \int \frac{f\left(z_{1}, z_{2} \mid S=1\right) \operatorname{Pr}(S=1)}{g\left(k_{1}\left(z_{1}\right)+k_{2}\left(z_{2}\right)\right)} d z_{2}=f_{1}\left(z_{1}\right), \\
& \int \frac{f\left(z_{1}, z_{2} \mid S=1\right) \operatorname{Pr}(S=1)}{g\left(k_{1}\left(z_{1}\right)+k_{2}\left(z_{2}\right)\right)} d z_{1}=f_{2}\left(z_{2}\right),
\end{aligned}
$$

with $f$ the joint density of $z_{1}$ and $z_{2}$ conditional on $S=1, f_{1}$ the marginal density of $z_{1}$ and $f_{2}$ the marginal density of $z_{2}$. These two integral equations connect $g$ with the first- and second-period representative sample, respectively. Note that the right-hand side of the second integral equation, $f_{2}$, cannot be identified from the

\footnotetext{
${ }^{1}$ In practice, the refreshment sample can be of two kinds. It can be randomly drawn from the second-period population, as in Hirano et al. (2001) and Bhattacharya (2008). In other cases, as in the CFM, the refreshment sample is not random but sampled so as to replace attritors and restore the representativity of the second-period sample. In either case, a representative second-period sample is available.
} 
unbalanced panel, because the $n_{12}$ units (stayers) that survive into the second period are not representative due to selective attrition: a refreshment sample is thus required to provide this quantity.

However, as discussed in Bhattacharya (2008), these two integral equations are of limited applicability for estimation: they cannot be solved to yield a closed-form solution. The key insight of Bhattacharya (2008) is to show that Hirano et al.'s (2001) integral equations are equivalent to the following conditional moments:

$$
\begin{aligned}
& E\left[\frac{S}{g\left(k_{1}\left(z_{1}\right)+k_{2}\left(z_{2}\right)\right)}-1 \mid R_{1}=1, z_{1}\right]=0 \text { for all } z_{1}, \\
& E\left[\frac{S}{g\left(k_{1}\left(z_{1}\right)+k_{2}\left(z_{2}\right)\right)}-1 \mid R_{2}=1, z_{2}\right]=0 \text { for all } z_{2},
\end{aligned}
$$

where the dummy $R_{1}$ indicates whether a unit belongs to the first-period representative sample, and the dummy $R_{2}$ indicates whether a unit belongs to the second-period representative sample. The moment interpretation allows Bhattacharya (2008) to provide a sieve-based method of estimating the attrition function.

We also conduct specification tests for both the MAR and Hausman and Wise (HW) attrition functions using unconditional (parametric) moments derived from equations (B.3) and (B.4). The null hypothesis for MAR is

$$
H_{0}: \quad g\left(z_{1} \beta_{1}\right) \text { satisfies both conditional moments (B.3) and (B.4), }
$$

while for HW it is

$$
H_{0}: \quad g\left(z_{2} \beta_{2}\right) \text { satisfying both conditional moments (B.3) and (B.4). }
$$

The Hansen-Sargan test statistics reject both of these null hypotheses. However, the expected Jacobian matrix might not have full rank, so that our inference may not be robust to arbitrary unconditional moments, especially when there are too many 
unconditional moments included. We leave this for future research.

\section{B.2.2 Estimation via sieves}

Following Ai and Chen (2007), we use sieves to approximate (i) the conditional moments $m_{0}\left(x_{1}, x_{2}, \alpha\right), m_{1}\left(z_{1}, \alpha\right)$ and $m_{2}\left(z_{2}, \alpha\right)$; and (ii) the unknown functions $k_{1}$ and $k_{2}$. Then we construct the SMD criterion function to minimize with respect to lin-

ear subspaces of $\alpha$. Let $\left\{p_{0 l}\left(x_{1}, x_{2}\right), p_{1 l}\left(z_{1}\right), p_{2 l}\left(z_{2}\right)\right\}_{l=1, \ldots, K_{n}}$ be known sieve functions whose number $K_{n}$ grows with the sample size. Also define:

$$
\begin{aligned}
& p_{0}^{K_{n}}\left(x_{1 i}, x_{2 i}\right)= \begin{cases}\left\{p_{0 l}\left(x_{1 i}, x_{2 i}\right)\right\}_{l=1, \ldots, K_{n}} & \text { if } S_{i}=1, \\
0 & \text { otherwise, }\end{cases} \\
& p_{1}^{K_{n}}\left(z_{1 i}\right)= \begin{cases}\left\{p_{1 l}\left(z_{1 i}\right)\right\}_{l=1, \ldots, K_{n}} & \text { if } R_{1 i}=1, \\
0 & \text { otherwise },\end{cases} \\
& p_{2}^{K_{n}}\left(z_{2 i}\right)= \begin{cases}\left\{p_{2 l}\left(z_{2 i}\right)\right\}_{l=1, \ldots, K_{n}} & \text { if } R_{2 i}=1, \\
0 & \text { otherwise }\end{cases}
\end{aligned}
$$

and let

$$
P_{s}=\left\{p_{0}^{K_{n}}\left(x_{1 i}, x_{2 i}\right), p_{1}^{K_{n}}\left(z_{1 i}\right), p_{2}^{K_{n}}\left(z_{2 i}\right)\right\}_{i=1,2, \ldots, n}^{T}
$$

The least-squares sieve estimates of $m_{0}\left(x_{1}, x_{2}, \alpha\right), m_{1}\left(z_{1}, \alpha\right)$ and $m_{2}\left(z_{2}, \alpha\right)$ are given 
by

$$
\begin{aligned}
& \widehat{m}_{0}\left(x_{1 j}, x_{2 j}, \alpha\right) \equiv \sum_{i=1}^{n} S_{i} \frac{\phi\left(z_{1 i}, z_{2 i}, \beta_{0}\right)}{\left.g\left(k_{1}\left(z_{1 i}\right)+k_{2}\left(z_{2 i}\right)\right)\right)} p_{0}^{K_{n}}\left(x_{1 i}, x_{2 i}\right)^{\prime}\left(P_{0}^{\prime} P_{0}\right)^{-1} p_{0}^{K_{n}}\left(x_{1 j}, x_{2 j}\right), \\
& \widehat{m}_{1}\left(z_{1 j}, \alpha\right) \equiv \sum_{i=1}^{n} R_{1 i} \frac{S_{i}}{g\left(k_{1}\left(z_{1 i}\right)+k_{2}\left(z_{2 i}\right)\right)} p_{1}^{K_{n}}\left(z_{1 i}\right)^{\prime}\left(P_{1}^{\prime} P_{1}\right)^{-1} p_{1}^{k_{n}}\left(z_{1 j}\right)-1 \\
& \widehat{m}_{2}\left(z_{2 j}, \alpha\right) \equiv \sum_{i=1}^{n} \frac{n_{2}}{n_{1}} R_{1 i} \frac{S_{i}}{g\left(k_{1}\left(z_{1 i}\right)+k_{2}\left(z_{2 i}\right)\right)} p_{2}^{K_{n}}\left(z_{2 i}\right)^{\prime}\left(P_{2}^{\prime} P_{2}\right)^{-1} p_{2}^{k_{n}}\left(z_{2 j}\right)-1
\end{aligned}
$$

The expression for $\widehat{m}_{2}\left(z_{2 j}, \alpha\right)$ is derived in the following fashion. Let $\gamma_{2}^{\prime} p_{2}^{K_{n}}\left(z_{2}\right)$ denote a linear approximation of $E\left[\frac{S}{g(\cdot)} \mid R_{2}=1, z_{2}\right]$ where

$$
\begin{aligned}
\gamma_{2}^{\prime} & =E\left[\frac{S}{g\left(k_{1}\left(z_{1}\right)+k_{2}\left(z_{2}\right)\right)} p_{2}^{K_{n}}\left(z_{2}\right)^{\prime} \mid R_{2}=1\right] E\left[p_{2}^{K_{n}}\left(z_{2}\right) p_{2}^{K_{n}}\left(z_{2}\right)^{\prime} \mid R_{2}=1\right]^{-1} \\
& =E\left[\frac{S}{g\left(k_{1}\left(z_{1}\right)+k_{2}\left(z_{2}\right)\right)} p_{2}^{K_{n}}\left(z_{2}\right)^{\prime} \mid R_{1}=1\right] E\left[p_{2}^{K_{n}}\left(z_{2}\right) p_{2}^{K_{n}}\left(z_{2}\right)^{\prime} \mid R_{2}=1\right]^{-1} \\
& =\frac{E\left(R_{2}\right)}{E\left(R_{1}\right)} E\left[R_{1} \frac{S}{g\left(k_{1}\left(z_{1}\right)+k_{2}\left(z_{2}\right)\right)} p_{2}^{K_{n}}\left(z_{2}\right)^{\prime}\right] E\left[R_{2} p_{2}^{K_{n}}\left(z_{2}\right) p_{2}^{K_{n}}\left(z_{2}\right)^{\prime}\right]^{-1},
\end{aligned}
$$

and the second equality is due to the assumption that the population has not changed from period 1 to period 2 .

Notice that we are writing the estimated conditional moments under the common index $i=1, \ldots n$, even though they are in fact based on different samples: the balanced, unbalanced or refreshment. This notation will allow us to exactly follow Ai and Chen (2007) for calculating the asymptotic variance.

\section{B.2.3 Asymptotic normality of SMD}

We first define the rescaled $l=1, \ldots, d_{\beta}$ pathwise derivatives of the $m_{s}$ moments where $s=0,1,2$ as $D_{s l}\left(\cdot, w_{l}\right)=\frac{\partial m_{s}(\cdot, \alpha)}{\partial \beta_{l}}-\frac{d m_{s}(\cdot, \alpha)}{d \kappa}\left[w_{l}\right]$, where $w_{l}$ is the direct sum of function 
spaces of $z_{1}$ and $z_{2}$ :

$$
\begin{aligned}
D_{0 l}\left(x_{1}, x_{2}, w_{l}\right) \equiv & E\left[\frac{\partial \phi\left(z_{1}, z_{2}, \beta_{0}\right) / \partial \beta_{l}}{g\left(k_{1}\left(z_{1}\right)+k_{2}\left(z_{2}\right)\right)}\right. \\
& \left.+\frac{\phi\left(z_{1}, z_{2}, \beta_{0}\right) g^{\prime}\left(k_{1}\left(z_{1}\right)+k_{2}\left(z_{2}\right)\right)}{g^{2}\left(k_{1}\left(z_{1}\right)+k_{2}\left(z_{2}\right)\right)} w_{l}\left(z_{1}, z_{2}\right) \mid S=1, x_{1}, x_{2}\right], \\
D_{1 l}\left(z_{1}, w_{l}\right) \equiv & \equiv\left[\frac{g^{\prime}\left(k_{1}\left(z_{1}\right)+k_{2}\left(z_{2}\right)\right)}{g\left(k_{1}\left(z_{1}\right)+k_{2}\left(z_{2}\right)\right)} w_{l}\left(z_{1}, z_{2}\right) \mid R_{1}=1, z_{1}\right], \\
D_{2 l}\left(z_{2}, w_{l}\right) & \equiv E\left[\frac{g^{\prime}\left(k_{1}\left(z_{1}\right)+k_{2}\left(z_{2}\right)\right)}{g\left(k_{1}\left(z_{1}\right)+k_{2}\left(z_{2}\right)\right)} w_{l}\left(z_{1}, z_{2}\right) \mid R_{2}=1, z_{2}\right],
\end{aligned}
$$

and let $D_{s}(\cdot, w) \equiv\left\{D_{s l}\left(\cdot, w_{l}\right)\right\}_{l=1, \ldots, d_{\beta}}^{\prime}$ for $s=0,1,2$.

Finally let $w^{*} \equiv\left(w_{1}^{*}, \ldots, w_{d_{\beta}}^{*}\right)$ where each individual element is

$$
\begin{aligned}
w_{l}^{*} \equiv \arg \min _{w_{l}}\left\{E_{x_{1}, x_{2}}\left[D_{0 l}\left(x_{1}, x_{2}, w_{l}\right)^{2} \mid S=1\right]+E_{z_{1}}\left[D_{1 l}\left(z_{1}, w_{l}\right)^{2} \mid R_{1}=1\right]\right. \\
\left.+E_{z_{2}}\left[D_{2 l}\left(z_{2}, w_{l}\right)^{2} \mid R_{2}=1\right]\right\} .
\end{aligned}
$$

According to Bhattacharya (2008) and Ai and Chen (2007), we have the following Riesz representation theorem for $\widehat{\beta}$ :

$$
\begin{aligned}
& \sqrt{n}\left(\widehat{\beta}-\beta_{0}\right) \\
& \quad=\Delta^{*-1} \frac{\sqrt{n}}{n} \sum_{i=1}^{n}\left[D_{0}\left(x_{1 i}, x_{2 i}, w^{*}\right)^{\prime} \rho_{0}\left(z_{1 i}, z_{2 i}, \alpha\right)+D_{1}\left(z_{1 i}, w^{*}\right)^{\prime} \rho_{1}\left(z_{1 i}, z_{2 i}, \alpha\right)\right. \\
& \left.\quad+D_{2}\left(z_{2 i}, w^{*}\right)^{\prime} \rho_{2}\left(z_{1 i}, z_{2 i}, \alpha\right)\right]+o_{p}(1)
\end{aligned}
$$

where

$$
\begin{aligned}
\rho_{0}\left(z_{1 i}, z_{2 i}, \alpha\right) & \equiv \frac{n}{n_{12}} S_{i} \frac{\phi\left(z_{1 i}, z_{2 i}, \beta_{0}\right)}{g\left(k_{1}\left(z_{1 i}\right)+k_{2}\left(z_{2 i}\right)\right)}, \\
\rho_{1}\left(z_{1 i}, z_{2 i}, \alpha\right) & \equiv \frac{n}{n_{1}} R_{1 i} \frac{S_{i}}{g\left(k_{1}\left(\left(z_{1 i}\right)+k_{2}\left(z_{2 i}\right)\right)\right.}-1, \\
\rho_{2}\left(z_{1 i}, z_{2 i}, \alpha\right) & \equiv \frac{n}{n_{1}} R_{1 i} \frac{S_{i}}{g\left(k_{1}\left(\left(z_{1 i}\right)+k_{2}\left(z_{2 i}\right)\right)\right.}-\frac{n}{n_{2}} R_{2 i},
\end{aligned}
$$


and

$$
\begin{aligned}
\Delta^{*} \equiv & E\left[D_{0}\left(x_{1}, x_{2}, w^{*}\right)^{\prime} D_{0}\left(x_{1}, x_{2}, w^{*}\right) \mid S=1\right]+E\left[D_{1}\left(z_{1}, w^{*}\right)^{\prime} D_{1}\left(z_{1}, w^{*}\right) \mid R_{1}=1\right] \\
& +E\left[D_{2}\left(z_{2}, w^{*}\right)^{\prime} D_{2}\left(z_{2}, w^{*}\right) \mid R_{2}=1\right] .
\end{aligned}
$$

Under the regularity assumptions given in Bhattacharya (2008) and Ai and Chen (2007), we have

$$
\sqrt{n}(\widehat{\beta}-\beta) \stackrel{d}{\rightarrow} N\left(0, \Delta^{*-1} \operatorname{Var}(\epsilon) \Delta^{*-1}\right)
$$

where $\epsilon \equiv D_{0}\left(x_{1}, x_{2}, w^{*}\right)^{\prime} \rho_{0}\left(z_{1}, z_{2}, \alpha\right)+D_{1}\left(z_{1}, w^{*}\right)^{\prime} \rho_{1}\left(z_{1}, z_{2}, \alpha\right)+D_{2}\left(z_{2}, w^{*}\right)^{\prime} \rho_{2}\left(z_{1}, z_{2}, \alpha\right)$.

\section{B.2.4 Asymptotic variance estimation}

To estimate $\Delta^{*}$ and $\operatorname{Var}(\epsilon)$, we must obtain the sieve approximated $\hat{w}^{*} \equiv\left(\hat{w}_{1}^{*}, \ldots, \hat{w}_{d_{\beta}}^{*}\right)$ of $w^{*}$ by minimizing:

$$
\frac{1}{n} \sum_{i=1}^{n}\left\{\frac{n}{n_{12}} S_{i} \widehat{D}_{0 l}\left(x_{1 i}, x_{2 i}, w_{l}\right)^{2}+\frac{n}{n_{1}} R_{1 i} \widehat{D}_{1 l}\left(z_{1 i}, w_{l}\right)^{2}+\frac{n}{n_{2}} R_{2 i} \widehat{D}_{2 l}\left(z_{2 i}, w_{l}\right)^{2}\right\}
$$

for $l=1, \ldots, d_{\beta}$, where

$$
\begin{aligned}
& \widehat{D}_{0 l}\left(x_{1 j}, x_{2 j}, w_{l}\right) \\
& \quad=\sum_{i=1}^{n} S_{i}\left(\frac{\partial \phi\left(z_{1 i}, z_{2 i}, \widehat{\beta}\right) / \partial \widehat{\beta}_{l}}{g\left(\widehat{k_{1}}\left(z_{1 i}\right)+\widehat{k_{2}}\left(z_{2 i}\right)\right)}+\frac{\phi\left(z_{1 i}, z_{2 i}, \widehat{\beta}\right) g^{\prime}\left(\widehat{k_{1}}\left(z_{1 i}\right)+\widehat{k_{2}}\left(z_{2 i}\right)\right)}{g^{2}\left(\widehat{k_{1}}\left(z_{1 i}\right)+\widehat{k_{2}}\left(z_{2 i}\right)\right)} w_{l}\left(z_{1 i}, z_{2 i}\right)\right) \\
& \quad \times p_{0}^{k_{n}}\left(x_{1 i}, x_{2 i}\right)^{\prime} \times\left(P_{0}^{\prime} P_{0}\right)^{-1} p_{0}^{k_{n}}\left(x_{1 j}, x_{2 j}\right),
\end{aligned}
$$


and

$$
\begin{aligned}
& \widehat{D}_{1 l}\left(z_{1 j}, w_{l}\right)=\sum_{i=1}^{n} R_{1 i} \frac{S_{i} g^{\prime}\left(\widehat{k_{1}}\left(z_{1 i}\right)+\widehat{k_{2}}\left(z_{2 i}\right)\right)}{g^{2}\left(\widehat{k_{1}}\left(z_{1 i}\right)+\widehat{k_{2}}\left(z_{2 i}\right)\right)} w_{l}\left(z_{1 i}, z_{2 i}\right) p_{1}^{k_{n}}\left(z_{1 i}\right)^{\prime}\left(P_{1}^{\prime} P_{1}\right)^{-1} p_{1}^{k_{n}}\left(z_{1 j}\right), \\
& \widehat{D}_{2 l}\left(z_{2 j}, w_{l}\right)=\sum_{i=1}^{n} \frac{n_{2}}{n_{1}} R_{1 i} \frac{S_{i} g^{\prime}\left(\widehat{k_{1}}\left(z_{1 i}\right)+\widehat{k_{2}}\left(z_{2 i}\right)\right)}{g^{2}\left(\widehat{k_{1}}\left(z_{1 i}\right)+\widehat{k_{2}}\left(z_{2 i}\right)\right)} w_{l}\left(z_{1 i}, z_{2 i}\right) p_{2}^{k_{n}}\left(z_{2 i}\right)^{\prime}\left(P_{2}^{\prime} P_{2}\right)^{-1} p_{2}^{k_{n}}\left(z_{2 j}\right) .
\end{aligned}
$$

Finally, we estimate $\operatorname{Var}(\epsilon)$ by $\frac{1}{n} \sum_{i=1}^{n} \widehat{\epsilon}_{i} \widehat{\epsilon}_{i}^{\prime}$ where $\widehat{\epsilon}_{i} \equiv \widehat{D}_{0}\left(x_{1 i}, x_{2 i}, \widehat{w}^{*}\right)^{\prime} \rho_{0}\left(z_{1 i}, z_{2 i}, \hat{\alpha}\right)+$ $\widehat{D}_{1}\left(z_{1 i}, \widehat{w}^{*}\right)^{\prime} \rho_{1}\left(z_{1 i}, z_{2 i}, \hat{\alpha}\right)+\widehat{D}_{2}\left(z_{2 i}, \widehat{w}_{l}^{*}\right)^{\prime} \rho_{2}\left(z_{1 i}, z_{2 i}, \hat{\alpha}\right)$.

The SMD estimates obtained on the two-year panels with and without attrition correction are summarized in Tables B.1 and B.2. In addition, the effects of the attrition correction are plotted in Figures B.2 to B.5.

\section{B.3 Practical Implementation}

\section{B.3.1 Sieve space and $g(\cdot)$ function}

Power series are used to approximate both the conditional moments and the infinite

dimensional parameter(s) inside the attrition function. To choose the number of terms included in the power series, a trade-off has to be made between the sieve space's flexibility and the associated computational burden.

All results reported in Chapter 1 and its Technical Appendix are obtained using terms up to third and fourth degrees to approximate the conditional moments and $k$ functions, respectively. We also verify that results hardly change by increasing/decreasing the degrees. Also note that, to avoid collinearity issues, we use orthogonal polynomials rather than raw polynomials.

Finally, the $g(\cdot)$ function used in our application is the standard normal cumulative distribution function $(\mathrm{CDF})$. Other choices for the $g(\cdot)$ function gives similar results, such as the logistic CDF or an approximated $1+e^{-(\cdot)}$ for $1 / \Phi(\cdot)$ to avoid the zero 
denominator problem.

\section{B.3.2 Optimization procedure}

Minimization involved in the parameters and asymptotic variance estimations are carried out with the general-purpose optimization function optim from package stats in R. We utilize the Nelder-Mead algorithm with a relative convergence tolerance $1 e-8$ (default). ${ }^{2}$ Whenever possible, the computations are carried out in parallel using the R package SNOW on EDITH or the Bank of Canada High Performance Cluster. For more details on the implementation of SNOW, refer to Tierney et al. (2013).

\section{B.3.3 Estimation algorithm}

STEP 1: Compute a collection of initial values via parametric estimation of the firstdifference equation $\phi(\cdot)$ and attrition function $g(\kappa(\cdot))$.

STEP 2: Minimize the objective function $Q(\beta, \kappa)$ for each set of initial values. Obtain a first set of estimates $(\hat{\beta}, \hat{\kappa})$ as the solution that has the smallest objective function value.

STEP 3: Compute $\hat{\Sigma}$, the standard errors of the finite dimensional parameter estimates $\hat{\beta}$.

STEP 4: Define two new vectors of initial values, $(\hat{\beta} \pm 2 \hat{\Sigma}, \hat{\kappa})$. Minimize the objective function to obtain two additional sets of estimates, $\left(\hat{\beta}_{(+)}, \hat{\kappa}_{(+)}\right)$and $\left(\hat{\beta}_{(-)}, \hat{\kappa}_{(-)}\right)$.

$\operatorname{STEP} 5:$ Let $Q\left(\hat{\beta}^{*}, \hat{\kappa}^{*}\right)=\min \left\{Q(\hat{\beta}, \hat{\kappa}), Q\left(\hat{\beta}_{(+)}, \hat{\kappa}_{(+)}\right), Q\left(\hat{\beta}_{(-)}, \hat{\kappa}_{(-)}\right)\right\}$. If $\left(\hat{\beta}^{*}, \hat{\kappa}^{*}\right)=(\hat{\beta}, \hat{\kappa})$, the algorithm has converged. Otherwise, $\operatorname{set}(\hat{\beta}, \hat{\kappa}) \equiv\left(\hat{\beta}^{*}, \hat{\kappa}^{*}\right)$ and go back to Step 3. Repeat Steps 3 to 5 until the algorithm converges.

\footnotetext{
${ }^{2}$ Nelder-Mead's method works best in Bhattacharya's (2008) simulations. Other optimization routines give similar results. Results are also robust to scaling changes.
} 


\section{B.4 Additional Results on Multiple Payment In- novations}

Table 1.3 of Chapter 1 tabulates the joint adoption of CTC and SVC. Of the 16 cells, only 4 cells involve double-switchers (N-U;U or U;NU for both CTC and SVC). To ascertain the quantitative importance of this joint correlation we estimate the impacts of CTC and SVC jointly. We conduct the estimation with OLS and FD estimators as these are two polar opposites. We find that the estimates are similar to single-innovation regressions. This result is not surprising since the proportion of double-switchers is small. We present the results in Table B.4

\section{B.5 Additional Results on Item Nonresponse}

The incidence of item nonresponse in the CFM data is described in Table B.5. The first column provides, for each variable, the fraction of observations that are missing due to item nonresponse. The second column presents a cumulative aggregate item nonresponse rate which is computed as the ratio of incomplete observations with at least one variable missing in a set of variables. Item nonresponse rates are similar when considering each year separately, and just slightly higher for two-year and threeyear stayers.

Table B.6 is an extended version of Table 1.7 of Chapter 1. In its last two columns, the GMM estimate $\mathcal{M}_{A N}^{2, G M M}$ is obtained with attrition correction while $\mathcal{M}_{A N, N R}^{2, G M M}$ is obtained with both attrition and item nonresponse correction. In both cases, we employ a parametric approach instead of the non-parametric sieve approach of $\mathcal{M}_{A N}^{2}$. Comparing $\mathcal{M}_{A N}^{2, G M M}$ and $\mathcal{M}_{A N}^{2}$, we observe that the parametric and non-parametric estimation results are similar (but closer to zero in the parametric case). Chen and Liao (2015) show that sieve semi-parametric two-step GMM inference procedures are 
numerically equivalent to the ones computed with a parametric first step. Although their result is based on two-step GMM, we expect the similar arguments to be true for our one-step GMM as well.

In our empirical analysis, we assume that item nonresponse is MAR (as described above) so as to generate comparable results between our reweighting approach and imputation approach. Si et al. (2015) using Bayesian methods also assume MAR when imputing missing values due to item nonresponse but assume AN for imputing missingness due to the unit nonresponse. For CTC there is essentially no difference between the $\mathcal{M}_{A N}^{2, G M M}$ and $\mathcal{M}_{A N, N R}^{2, G M M}$ estimates. For SVC, however, $\hat{\beta}$ is larger in absolute magnitude when correcting for item nonresponse in addition to attrition. Although item nonresponse correction decreases SVC estimates away from zero, for cash in value $\hat{\beta}$ remains insignificant at usual confidence level. Therefore, we conclude that our empirical results are robust to item nonresponse correction: new estimates still show non significant impact of the use of CTC on cash usage, both in terms of volume and value, at usual significance level. The impact of SVC is significant on cash in volume only, with an estimated effect around 2 percent.

In Table B.7, we further compare the results from the incomplete data set with item nonresponse to those obtained on imputed data sets. Imputing the data set has little impact on the Pooled OLS estimates, while it leads to a downward decrease in the FD and $\mathcal{M}_{A N}^{2}$ estimates for both CTC and SVC. ${ }^{3}$ As for the effect of attrition correction on the panel estimates obtained on imputed samples (Columns 5 and 6), it is of the same order of magnitude as what is observed for the original data set with item nonresponse (Columns 2 and 3).

Under attrition correction, the two alternative approaches for handling item non-

\footnotetext{
${ }^{3}$ The numbers in Table B.7 are based on one imputed data set. In order to incorporate the effects of imputations into the variance estimation, we also compute the Pooled OLS and FD estimates on 100 imputed samples, and $\mathcal{M}_{A N}^{2}$ estimates on 3 imputed samples (the latter estimation being very computationally intensive). We find that our empirical results are not materially affected by the number of imputations.
} 
response (reweighting and imputation) have analogous impacts on $\hat{\beta}$ for SVC, but dissimilar effects as far as CTC is concerned. Recall that the reweighting approach uses the inverse of the conditional probability of complete response (no item nonresponse) to reweight the panel data set. On the other hand, imputation fills in missing values by modeling each of the variables that have item nonresponse. Horowitz and Manski (1998) note that estimates from using reweighting are usually different from those using imputations, because reweighting modifies the observed data but imputation takes the observed data as given. Unless weights are computed in particular ways, estimates from reweighting and imputation are usually different. 


\section{References}

Ai, C. And X. Chen (2007): "Estimation of possibly misspecified semiparametric conditional moment restriction models with different conditioning variables," Journal of Econometrics, 141, 5-43.

Bhattacharya, D. (2008): "Inference in panel data models under attrition caused by unobservables," Journal of Econometrics, 144, 430-446.

Chen, X. And Z. Liao (2015): "Sieve semiparametric two-step $\{$ GMM $\}$ under weak dependence," Journal of Econometrics, 189, 163 - 186.

Hirano, K., G. W. Imbens, G. Ridder, and D. B. Rubin (2001): "Combining panel data sets with attrition and refreshment samples," Econometrica, 69, 16451659.

Horowitz, J. L. And C. F. Manski (1998): "Censoring of outcomes and regressors due to survey nonresponse: Identification and estimation using weights and imputations," Journal of Econometrics, 84, 37 - 58.

Si, Y., J. P. Reiter, And D. S. Hillygus (2015): "Semi-parametric selection models for potentially non-ignorable attrition in panel studies with refreshment samples," Political Analysis, 23, 92-112.

Tierney, L., A. J. Rossini, N. Li, And H. Sevcikova (2013): snow: Simple Network of Workstations, r package version 0.3-13. 
TABLE B.1: Cash ratio regressions with attrition correction - CTC

\begin{tabular}{|c|c|c|c|c|c|c|}
\hline & \multirow{2}{*}{$\begin{array}{c}\text { No correction } \\
\mathcal{M}_{N C}^{\bullet}\end{array}$} & \multicolumn{5}{|c|}{ Attrition Correction } \\
\hline & & $\mathcal{M}_{M A R}^{1}$ & $\mathcal{M}_{H W}^{1}$ & $\mathcal{M}_{A N 1}^{1}$ & $\mathcal{M}_{A N 2}^{1}$ & $\mathcal{M}_{A N}^{2}$ \\
\hline \multicolumn{7}{|l|}{ 2010-11 panel } \\
\hline$\hat{\beta}$ for cash volume & -0.007 & -0.008 & -0.010 & -0.009 & - & - \\
\hline s.e. & 0.0111 & 0.0112 & 0.0117 & 0.0118 & - & - \\
\hline$t$-stat & -0.61 & -0.70 & -0.85 & -0.79 & - & - \\
\hline$\hat{\beta}$ for cash value & 0.010 & 0.010 & 0.009 & 0.007 & - & - \\
\hline s.e. & 0.0100 & 0.0101 & 0.0099 & 0.0105 & - & - \\
\hline$t$-stat & 1.04 & 1.03 & 0.87 & 0.66 & - & - \\
\hline Observations & 3,500 & 6908 & 6908 & 10,518 & - & - \\
\hline Households & 1,750 & 5158 & 5158 & 8,768 & - & - \\
\hline \multicolumn{7}{|l|}{ 2011-12 panel } \\
\hline$\hat{\beta}$ for cash volume & -0.008 & -0.010 & -0.004 & -0.010 & 0.017 & - \\
\hline s.e. & 0.0110 & 0.0124 & 0.0131 & 0.0118 & 0.0169 & - \\
\hline$t$-stat & -0.72 & -0.79 & -0.32 & -0.86 & 0.98 & - \\
\hline$\hat{\beta}$ for cash value & -0.021 & -0.026 & -0.032 & -0.027 & -0.025 & - \\
\hline s.e. & 0.0100 & 0.0114 & 0.0127 & 0.0110 & 0.0179 & - \\
\hline$t$-stat & -2.11 & -2.25 & -2.53 & -2.44 & -1.41 & - \\
\hline Observations & 3,826 & 7,175 & 7,175 & 9,995 & 13,601 & - \\
\hline Households & 1,913 & 5,262 & 5,262 & 8,082 & 10,397 & - \\
\hline \multicolumn{7}{|c|}{ 2010-12 three-year panel } \\
\hline$\hat{\beta}$ for cash volume & 0.006 & - & - & - & - & 0.015 \\
\hline s.e. & 0.0108 & - & - & - & - & 0.0137 \\
\hline$t$-stat & 0.52 & - & - & - & - & 1.12 \\
\hline$\hat{\beta}$ for cash value & 0.006 & - & - & - & - & 0.002 \\
\hline s.e. & 0.0106 & - & - & - & - & 0.0125 \\
\hline$t$-stat & 0.61 & - & - & - & - & 0.14 \\
\hline Observations & 2,113 & - & - & - & - & 13,601 \\
\hline Households & 1,351 & - & - & - & - & 10,397 \\
\hline
\end{tabular}

Notes: $\hat{\beta}$ are point estimates, while s.e. are standard errors. $\mathcal{M}_{N C}^{\bullet}$ are sieve minimum distance estimators without attrition correction. All other estimators are sieve minimum distance estimators with attrition correction. Descriptions of the models estimated are available in Table B.3. 
TABLE B.2: Cash ratio regressions with corrections - SVC

\begin{tabular}{|c|c|c|c|c|c|c|}
\hline & \multirow{2}{*}{$\begin{array}{c}\text { No correction } \\
\mathcal{M}_{N C}^{\bullet}\end{array}$} & \multicolumn{5}{|c|}{ Attrition Correction } \\
\hline & & $\mathcal{M}_{M A R}^{1}$ & $\mathcal{M}_{H W}^{1}$ & $\mathcal{M}_{A N 1}^{1}$ & $\mathcal{M}_{A N 2}^{1}$ & $\mathcal{M}_{A N}^{2}$ \\
\hline \multicolumn{7}{|l|}{ 2010-11 panel } \\
\hline$\hat{\beta}$ for cash volume & -0.013 & -0.010 & -0.002 & -0.006 & - & - \\
\hline s.e. & 0.0090 & 0.0100 & 0.0100 & 0.0102 & - & - \\
\hline$t$-stat & -1.48 & -1.04 & -0.22 & -0.56 & - & - \\
\hline$\hat{\beta}$ for cash value & -0.008 & -0.007 & -0.006 & -0.004 & - & - \\
\hline s.e. & 0.0085 & 0.0093 & 0.0093 & 0.0095 & - & - \\
\hline$t$-stat & -0.94 & -0.80 & -0.63 & -0.43 & - & - \\
\hline Observations & 3,500 & 6908 & 6908 & 10,518 & - & - \\
\hline Households & 1,750 & 5158 & 5158 & 8,768 & - & - \\
\hline \multicolumn{7}{|l|}{ 2011-12 panel } \\
\hline$\hat{\beta}$ for cash volume & -0.030 & -0.028 & -0.034 & -0.027 & -0.023 & - \\
\hline s.e. & 0.0083 & 0.0085 & 0.0089 & 0.0083 & 0.0126 & - \\
\hline$t$-stat & -3.57 & -3.30 & -3.79 & -3.27 & -1.85 & - \\
\hline$\hat{\beta}$ for cash value & -0.011 & -0.007 & -0.010 & -0.006 & 0.002 & - \\
\hline s.e. & 0.0078 & 0.0082 & 0.0083 & 0.0081 & 0.0124 & - \\
\hline$t$-stat & -1.39 & -0.83 & -1.24 & -0.72 & 0.14 & - \\
\hline Observations & 3,826 & 7,175 & 7,175 & 9,995 & 13,601 & - \\
\hline Households & 1,913 & 5,262 & 5,262 & 8,082 & 10,397 & - \\
\hline \multicolumn{7}{|c|}{ 2010-12 three-year panel } \\
\hline$\hat{\beta}$ for cash volume & -0.020 & - & - & - & - & -0.022 \\
\hline s.e. & 0.0091 & - & - & - & - & 0.0099 \\
\hline$t$-stat & -2.22 & - & - & - & - & -2.19 \\
\hline$\hat{\beta}$ for cash value & -0.009 & - & - & - & - & -0.001 \\
\hline s.e. & 0.0085 & - & - & - & - & 0.0098 \\
\hline$t$-stat & -1.08 & - & - & - & - & -0.08 \\
\hline Observations & 2,113 & - & - & - & - & 13,601 \\
\hline Households & 1,351 & - & - & - & - & 10,397 \\
\hline
\end{tabular}

Notes: $\hat{\beta}$ are point estimates, while s.e. are standard errors. $\mathcal{M}_{N C}^{\bullet}$ are sieve minimum distance estimators without attrition correction. All other estimators are sieve minimum distance estimators with attrition correction. Descriptions of the models estimated are available in Table B.3. 
TABLE B.3: Description of the two-year panel models

\begin{tabular}{lcccc}
\hline \hline Model & EF & SF & Moments & Panels \\
\hline $\mathcal{M}_{N C}^{1}$ & $\phi_{t-1, t}$ & 1 & $M_{t-1, t}^{01}$ & $t=2011,2012$ \\
$\mathcal{M}_{A N 1}^{1}$ & $\phi_{t-1, t}$ & $g_{t-1, t}$ & $M_{t-1, t}^{01}, M_{t-1}^{11}, M_{t}^{21}$ & $t=2011,2012$ \\
$\mathcal{M}_{A N 2}^{1}$ & $\phi_{t-1, t}$ & $g_{t-2, t-1, t}$ & $M_{t-1, t}^{02}, M_{t-2}^{12}, M_{t-1}^{22}, M_{t}^{32}$ & $t=2012$ \\
$\mathcal{M}_{N C}^{2}$ & $\left\{\phi_{t-2, t-1}, \phi_{t-1, t}\right\}$ & $\{1,1\}$ & $\left\{M_{t-2, t-1}^{01}, M_{t-1, t}^{02}\right\}$ & $t=2012$ \\
$\mathcal{M}_{A N}^{2}$ & $\left\{\begin{array}{l}\phi_{t-2, t-1} \\
\phi_{t-1, t}\end{array}\right.$ & $\left\{\begin{array}{l}g_{t-2, t-1} \\
g_{t-2, t-1, t}\end{array}\right.$ & $\left\{\begin{array}{l}M_{t-2, t-1}^{01}, M_{t-2}^{11}, M_{t-1}^{21} \\
M_{t-1, t}^{02}, M_{t-2}^{12}, M_{t-1}^{22}, M_{t}^{32}\end{array}\right.$ & $t=2012$ \\
\hline \hline
\end{tabular}

The estimation function (EF) is defined as

$\phi_{t-1, t}: \quad \phi\left(z_{t-1}, z_{t}, \beta\right)=\Delta C R_{i t}=\beta \Delta P I_{i t}+\Delta X_{i t} \gamma+\Delta u_{i t}$.

The survival function (SF) is defined as

$$
\begin{array}{lll}
g_{t-1}: & \operatorname{Pr}\left(S_{t}=1\right) & \equiv g\left(k\left(z_{t-1}\right)\right), \\
g_{t}: & \operatorname{Pr}\left(S_{t}=1\right) & \equiv g\left(k\left(z_{t}\right)\right), \\
g_{t-1, t}: & \operatorname{Pr}\left(S_{t}=1\right) & \equiv g\left(k_{1}\left(z_{t-1}\right)+k_{2}\left(z_{t}\right)\right), \\
g_{t-2, t-1, t}: & \operatorname{Pr}\left(S_{t-1} S_{t}=1\right) & \equiv g\left(k_{1}\left(z_{t-2}\right)+k_{2}\left(z_{t-1}\right)+k_{3}\left(z_{t}\right)\right) .
\end{array}
$$

The moments are defined as

$$
\begin{aligned}
& M_{t-1, t}^{01}: m_{01}\left(x_{t-1}, x_{t}, \delta\right) \equiv E\left\{\frac{\phi\left(z_{t-1}, z_{t}, \beta\right)}{\operatorname{Pr}\left(S_{t}=1\right)} \mid S_{t}=1, x_{t-1}, x_{t}\right\}=0, \\
& M_{t-1}^{11}: \quad m_{11}\left(z_{t-1}, \delta\right) \equiv E\left\{\frac{S_{t}}{\operatorname{Pr}\left(S_{t}=1\right)}-1 \mid R_{t-1}=1, z_{t-1}\right\}=0, \\
& M_{t}^{21}: \quad m_{21}\left(z_{t}, \delta\right) \equiv E\left\{\frac{S_{t}}{\operatorname{Pr}\left(S_{t}=1\right)}-1 \mid R_{t}=1, z_{t}\right\}=0, \\
& M_{t-1, t}^{02}: m_{02}\left(x_{t-1}, x_{t}, \delta\right) \equiv E\left\{\frac{\phi\left(z_{t-1}, z_{t}, \beta\right)}{\operatorname{Pr}\left(S_{t-1} S_{t}=1\right)} \mid S_{t-1} S_{t}=1, x_{t-1}, x_{t}\right\}=0, \\
& M_{t-2}^{12}: \quad m_{12}\left(z_{t-2}, \delta\right) \equiv E\left\{\frac{S_{t-1} S_{t}}{\operatorname{Pr}\left(S_{t-1} S_{t}=1\right)}-1 \mid R_{t-2}=1, z_{t-2}\right\}=0, \\
& M_{t-1}^{22}: \quad m_{22}\left(z_{t-1}, \delta\right) \equiv E\left\{\frac{S_{t-1} S_{t}}{\operatorname{Pr}\left(S_{t-1} S_{t}=1\right)}-1 \mid R_{t-1}=1, z_{t-1}\right\}=0, \\
& M_{t}^{32}: \quad m_{32}\left(z_{t}, \delta\right) \equiv E\left\{\frac{S_{t-1} S_{t}}{\operatorname{Pr}\left(S_{t-1} S_{t}=1\right)}-1 \mid R_{t}=1, z_{t}\right\}=0 .
\end{aligned}
$$


TABLE B.4: Cash ratio regressions with CTC and SVC as regressors

\begin{tabular}{lccccc} 
& $\mathbf{2 0 1 0}$ & $\mathbf{2 0 1 1}$ & $\mathbf{2 0 1 2}$ & Pooled & Panel \\
& OLS & OLS & OLS & OLS & FD \\
\hline CTC & & & & & \\
beta for cash volume & -0.081 & -0.092 & -0.104 & -0.095 & 0.001 \\
s.e. & 0.0089 & 0.0081 & 0.0082 & 0.0055 & 0.0127 \\
t-statistics & -9.10 & -11.36 & -12.68 & -17.27 & 0.08 \\
& & & & & \\
beta for cash value & -0.082 & -0.084 & -0.096 & -0.088 & 0.004 \\
s.e. & 0.0072 & 0.0066 & 0.0068 & 0.0051 & 0.0126 \\
t-statistics & -11.39 & -12.73 & -14.12 & -17.25 & 0.32 \\
\hline SVC & & & & & \\
beta for cash volume & -0.013 & -0.018 & -0.028 & -0.019 & -0.018 \\
s.e. & 0.0069 & 0.0069 & 0.0072 & 0.0042 & 0.0085 \\
t-statistics & -1.88 & -2.61 & -3.89 & -4.52 & -2.12 \\
& & & & & \\
beta for cash value & -0.008 & -0.011 & -0.011 & -0.010 & -0.008 \\
s.e. & 0.0065 & 0.0064 & 0.0068 & 0.0040 & 0.0084 \\
t-statistics & -1.23 & -1.72 & -1.62 & -2.50 & -0.95 \\
\hline \hline
\end{tabular}

Notes: The effects are estimated in a single model with two dummies for CTC and SVC. 
TABLE B.5: Item nonresponse in the three-year panel

\begin{tabular}{rcc} 
& INR & Aggregate INR \\
\hline Cash ratio in volume & 28.7 & 28.7 \\
Cash ratio in value & 36.1 & 38.3 \\
CTC & 2.8 & 38.8 \\
SVC & 5.3 & 38.8 \\
Internet user & 2.6 & 40.5 \\
CC revolver & 12.1 & 43.7 \\
Rel. exp. share: groceries & 3.3 & 43.7 \\
Restaurants & 3.3 & 43.7 \\
Convenience stores & 3.3 & 43.7 \\
Recreation & 3.3 & 43.7 \\
Gas stations & 3.3 & 43.7 \\
\hline \hline
\end{tabular}

Notes: Numbers are in proportions, obtained from the 2010-2012 three-year panel. Item nonresponse (INR) rate is defined as the ratio of the number of observations missing due to item nonresponse to the total number of observations in the three-year panel (34,959 observations). The aggregate INR rate is the ratio of incomplete observations with at least one variable missing in a set of variables. For a variable in a given row, this set contains the variable itself as well as all the variables listed on previous rows. 


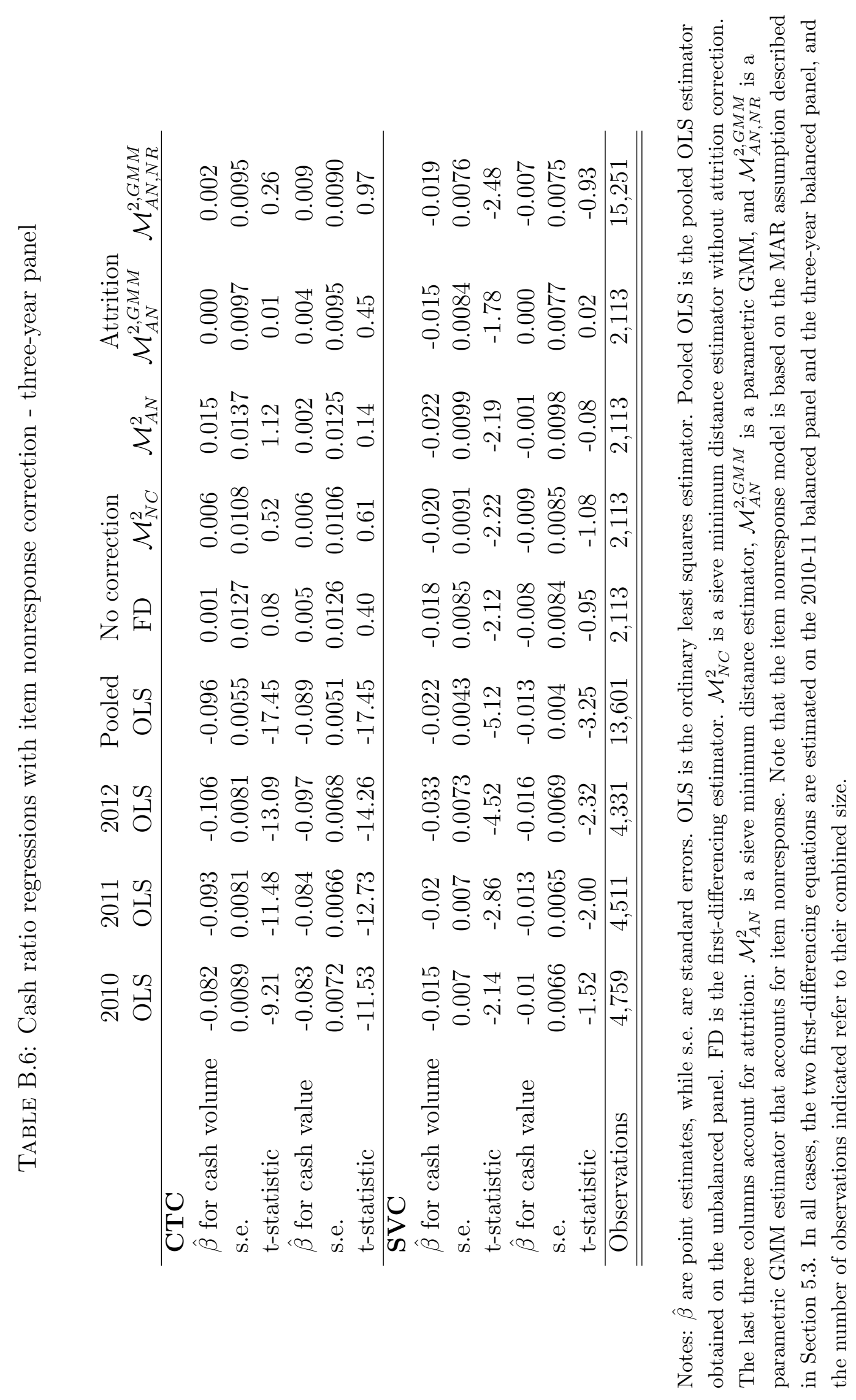


TABLE B.7: Cash ratio regressions under multiple-imputation for item nonresponse

\begin{tabular}{|c|c|c|c|c|c|c|}
\hline & \multicolumn{3}{|c|}{ With Item Nonresponse } & \multicolumn{3}{|c|}{ Multiple imputation } \\
\hline & \multicolumn{2}{|c|}{ No correction } & \multirow{2}{*}{$\begin{array}{c}\text { Attrition } \\
\mathcal{M}_{A N}^{2}\end{array}$} & \multicolumn{2}{|c|}{ No correction } & \multirow{2}{*}{$\begin{array}{c}\text { Attrition } \\
\mathcal{M}_{A N}^{2}\end{array}$} \\
\hline & P. OLS & FD & & P. OLS & FD & \\
\hline \multicolumn{7}{|l|}{ CTC } \\
\hline$\hat{\beta}$ for cash volume & -0.096 & 0.001 & 0.015 & -0.091 & -0.036 & -0.028 \\
\hline s.e. & 0.0055 & 0.0127 & 0.0137 & 0.0035 & 0.0073 & 0.0090 \\
\hline t-statistic & -17.45 & 0.08 & 1.12 & -26.03 & -4.82 & -3.08 \\
\hline$\hat{\beta}$ for cash value & -0.089 & 0.005 & 0.002 & -0.084 & -0.040 & -0.030 \\
\hline s.e. & 0.0051 & 0.0126 & 0.0125 & 0.0032 & 0.0070 & 0.0080 \\
\hline t-statistic & -17.45 & 0.40 & 0.14 & -26.10 & -5.74 & -3.78 \\
\hline \multicolumn{7}{|l|}{ SVC } \\
\hline$\hat{\beta}$ for cash volume & -0.022 & -0.018 & -0.022 & -0.024 & -0.025 & -0.020 \\
\hline s.e. & 0.0043 & 0.0085 & 0.0099 & 0.0028 & 0.0052 & 0.0067 \\
\hline t-statistic & -5.12 & -2.12 & -2.19 & -8.64 & -4.78 & -2.92 \\
\hline$\hat{\beta}$ for cash value & -0.013 & -0.008 & -0.001 & -0.013 & -0.013 & -0.009 \\
\hline s.e. & 0.004 & 0.0084 & 0.0098 & 0.0025 & 0.0050 & 0.0063 \\
\hline t-statistic & -3.25 & -0.95 & -0.08 & -5.28 & -2.66 & -1.39 \\
\hline Observations & 13,601 & 2,113 & 2,113 & 34,959 & 15,251 & 15,251 \\
\hline
\end{tabular}

Notes: $\hat{\beta}$ are point estimates, while s.e. are standard errors. Estimates in the first three columns are obtained without correcting for item nonresponse. Estimates in the last three columns are obtained when correcting for item nonresponse via imputation under the MAR assumption described in Section 5.3 with single imputation from the same imputed data. P. OLS is the pooled OLS estimator obtained on the unbalanced panel. FD is the first-differencing estimator. $\mathcal{M}_{A N}^{2}$ is the sieve minimum distance estimator with attrition correction. In all cases, the two first-differencing equations are estimated on the 2010-11 balanced panel and the three-year balanced panel, and the number of observations indicated refer to their combined size. 
Figure B.1: Two-period panel with refreshment sample

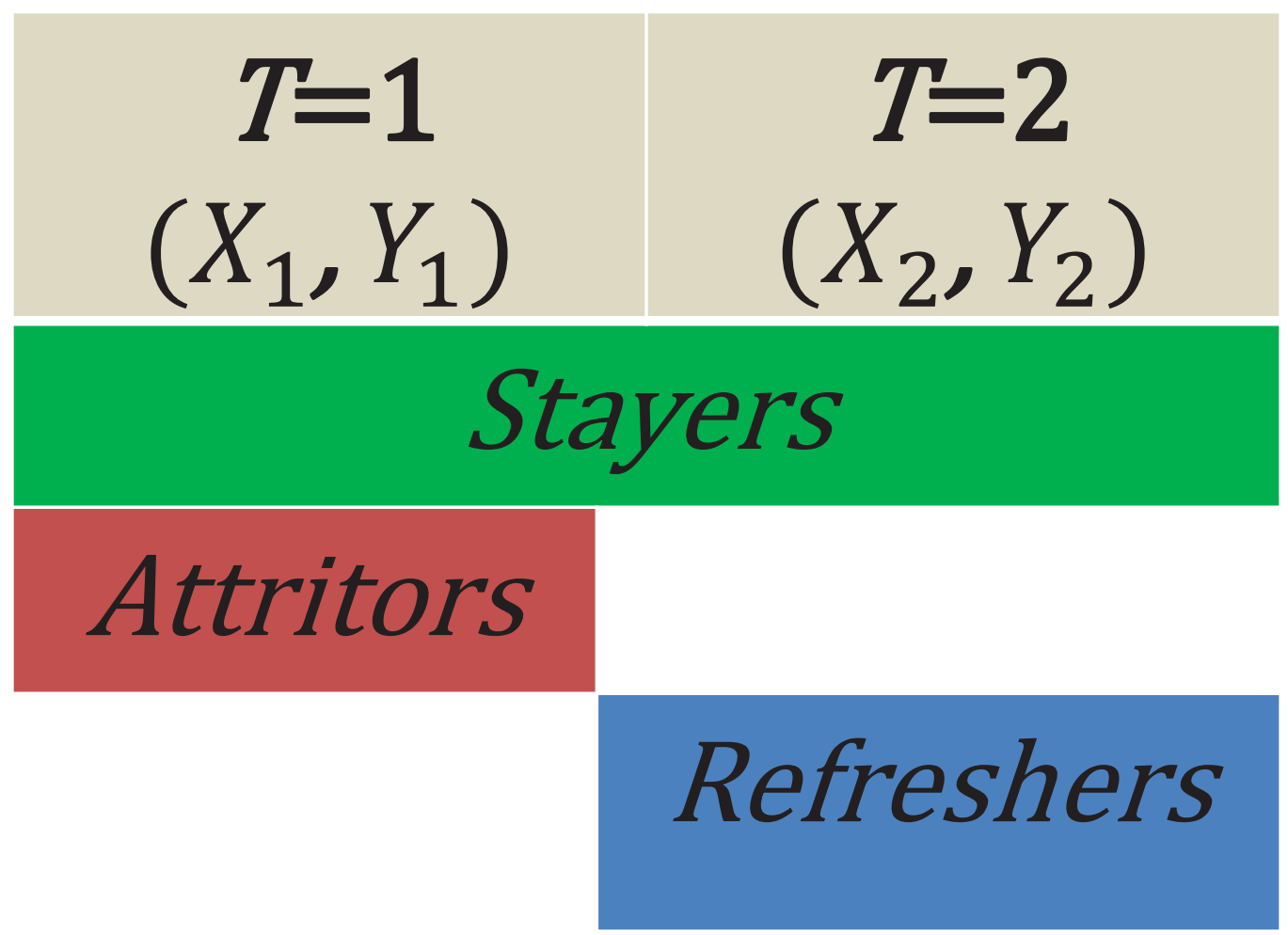


Figure B.2: CTC, attrition probability versus cash ratio in volume - two-year panels
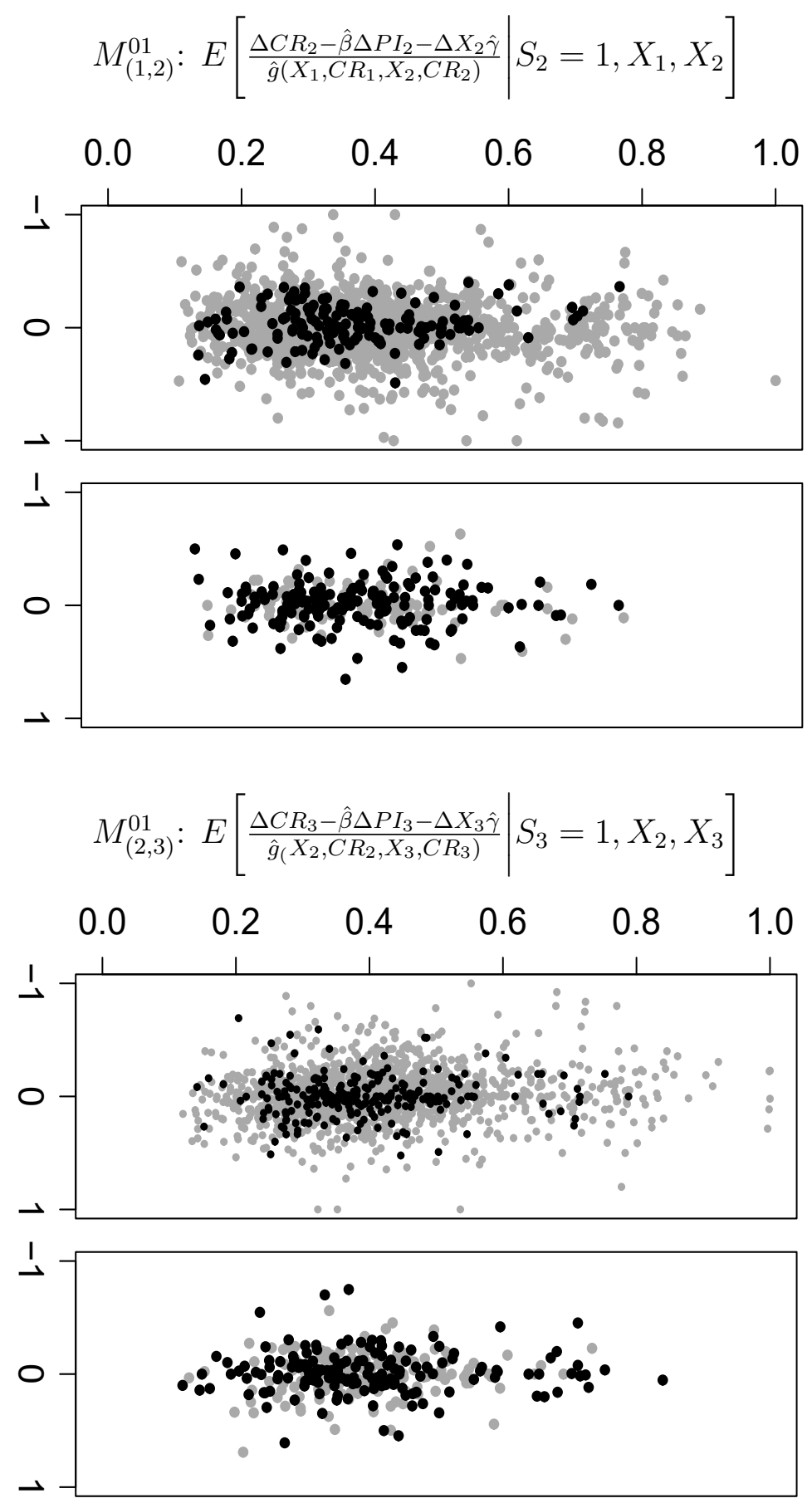

Notes: The estimated survival function, $\hat{g}(\cdot)$, is on the y-axis while the change in the cash ratio is on the x-axis. The functions $\hat{g}_{1,2}$ (from $M_{(1,2)}^{01}$ ) estimated on the 2010-11 panel and $\hat{g}_{2,3}$ (from $\left.M_{(2,3)}^{01}\right)$ estimated on the 2011-12 panel are depicted in the top and bottom panes, respectively. The left-side pane depicts: the never-users $(0,0)$ in grey and the always-users $(1,1)$ in black; the right-side pane contains: the stop-users $(1,0)$ in grey and the new-users $(0,1)$ in black. 
Figure B.3: CTC, attrition probability versus cash ratio in value - two-year panels
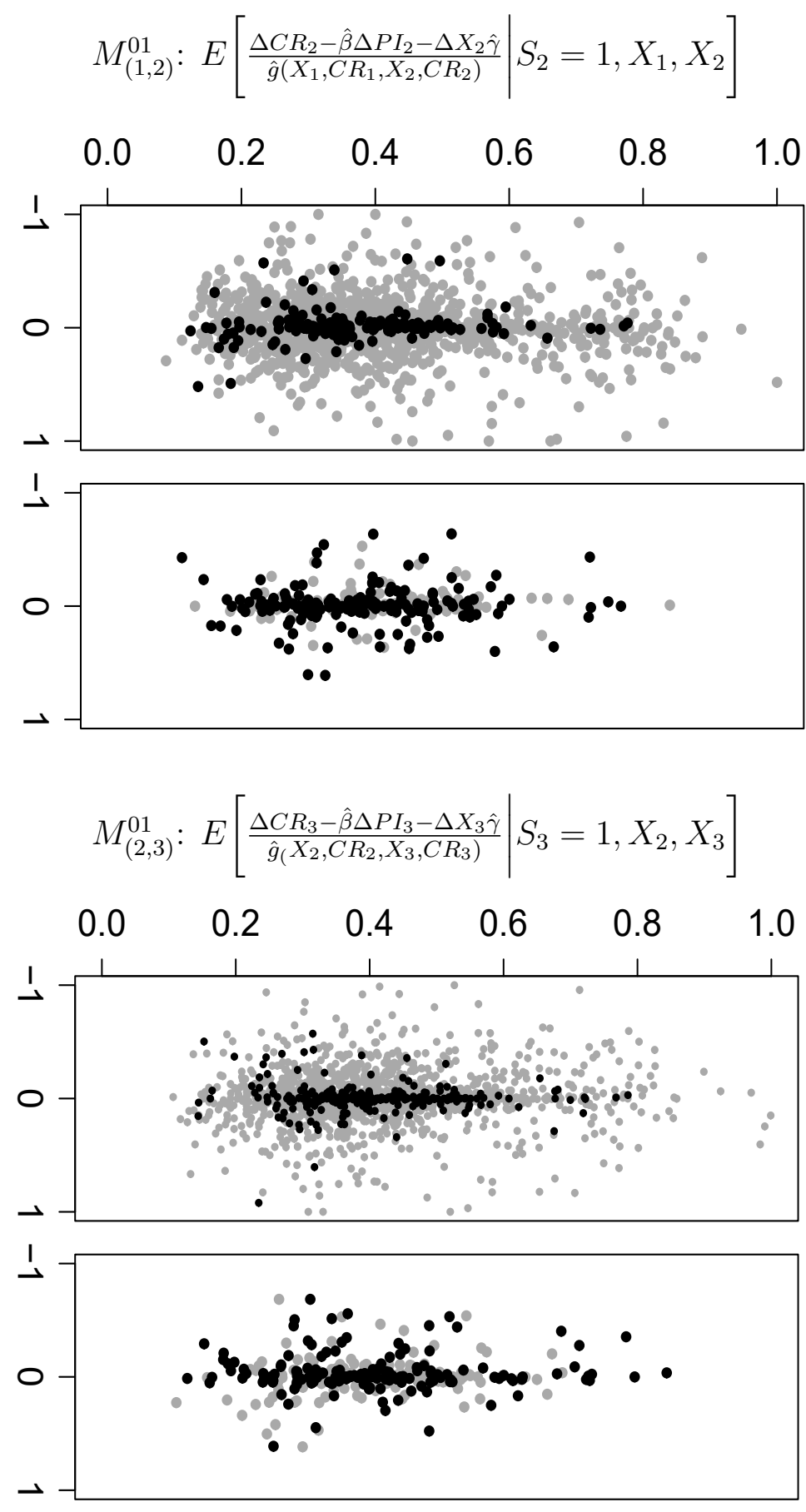

Notes: The estimated survival function, $\hat{g}(\cdot)$, is on the y-axis while the change in the cash ratio is on the x-axis. The functions $\hat{g}_{1,2}$ (from $M_{(1,2)}^{01}$ ) estimated on the 2010-11 panel and $\hat{g}_{2,3}$ (from $\left.M_{(2,3)}^{01}\right)$ estimated on the 2011-12 panel are depicted in the top and bottom panes, respectively. The left-side pane depicts: the never-users $(0,0)$ in grey and the always-users $(1,1)$ in black; the right-side pane contains: the stop-users $(1,0)$ in grey and the new-users $(0,1)$ in black. 
Figure B.4: SVC, attrition probability versus cash ratio in volume - two-year panels
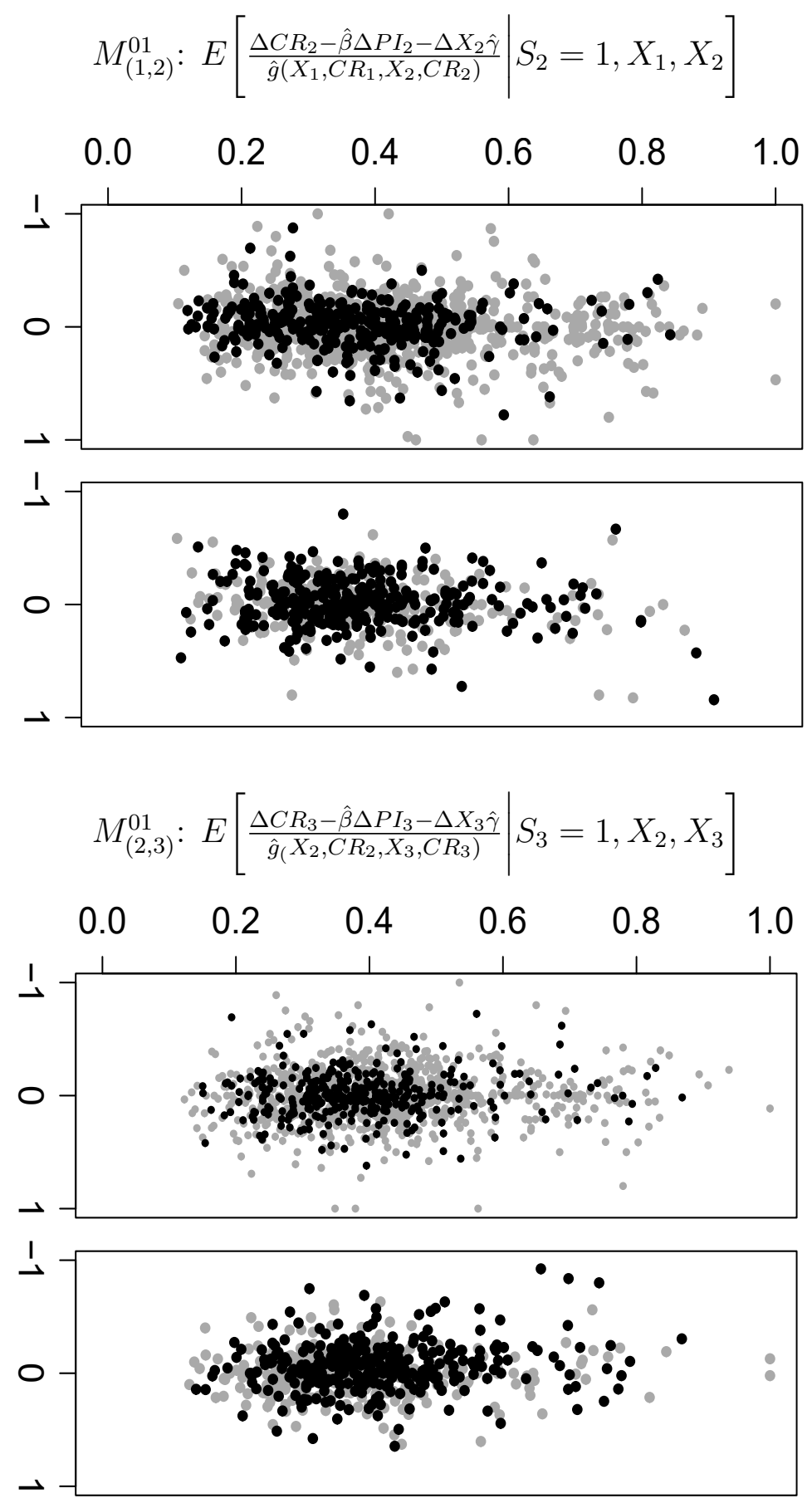

Notes: The estimated survival function, $\hat{g}(\cdot)$, is on the y-axis while the change in the cash ratio is on the x-axis. The functions $\hat{g}_{1,2}$ (from $M_{(1,2)}^{01}$ ) estimated on the 2010-11 panel and $\hat{g}_{2,3}$ (from $\left.M_{(2,3)}^{01}\right)$ estimated on the 2011-12 panel are depicted in the top and bottom panes, respectively. The left-side pane depicts: the never-users $(0,0)$ in grey and the always-users $(1,1)$ in black; the right-side pane contains: the stop-users $(1,0)$ in grey and the new-users $(0,1)$ in black. 
Figure B.5: SVC, attrition probability versus cash ratio in value - two-year panels
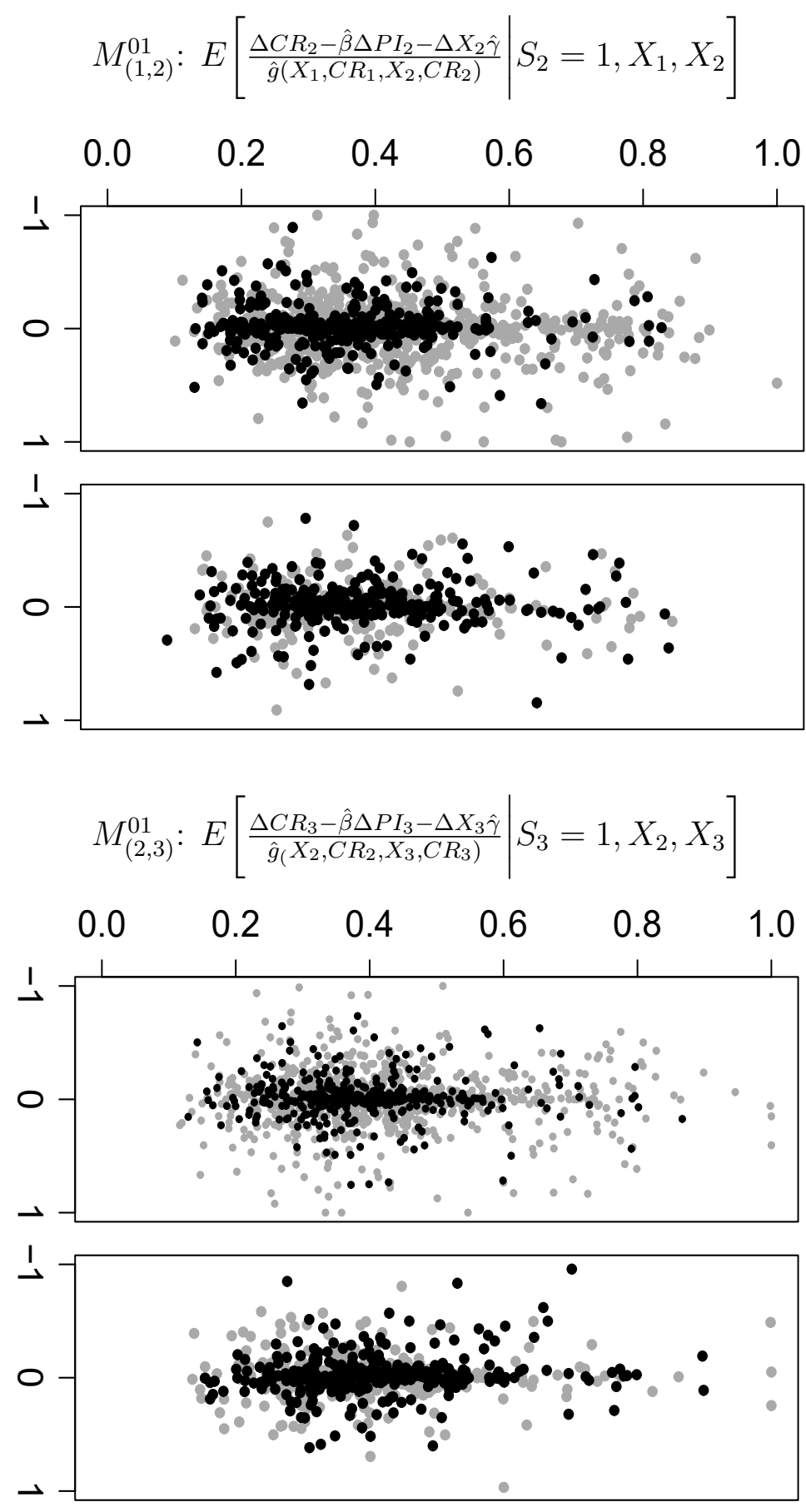

Notes: The estimated survival function, $\hat{g}(\cdot)$, is on the y-axis while the change in the cash ratio is on the x-axis. The functions $\hat{g}_{1,2}$ (from $M_{(1,2)}^{01}$ ) estimated on the 2010-11 panel and $\hat{g}_{2,3}$ (from $\left.M_{(2,3)}^{01}\right)$ estimated on the 2011-12 panel are depicted in the top and bottom panes, respectively. The left-side pane depicts: the never-users $(0,0)$ in grey and the always-users $(1,1)$ in black; the right-side pane contains: the stop-users $(1,0)$ in grey and the new-users $(0,1)$ in black. 


\section{Chapter 2}

\section{A Look Inside the Box: Combining}

Aggregate and Marginal

\section{Distributions to Identify Joint}

\section{Distributions}

\section{$2.1 \quad$ Introduction}

In multiple contexts, the variables of interest to a research question are not all available in one single data set. Depending on the data at hand and the assumptions one is ready to make, data combination might then offer avenues to solve the identification problem at stake. As described in Ridder and Moffitt (2007), a detailed survey of the recent data combination literature in statistics and economics, most sample combination strategies rely on the presence of common units and/or common variables across samples to be combined.

Let $X, Y, Z$ denote variables that are defined for each member of a population, and let the joint distribution of $X, Y$ be the object of interest. The usual framework 
considered is that where one sample contains variables $X, Z$ and a second sample contains variables $Y, Z$ - that is, random samples from overlapping (in variables) marginal distributions are available. If a substantial fraction of units appear in both samples and can, at least to a certain degree, be identified by the common variables $Z$, then the samples can be merged via matching techniques. If the two samples are independent (no units in common), variables in common can be called upon to formulate conditional independence or exclusion restriction assumptions that assure identification.

In practice, the matching of data sets with common units applies only in very specific situations, and mainly concerns administrative records. While the common variable requirement is more commonly met, it might fail to provide valid identification assumptions. For example, if the set of common variables $Z$ is small, there might simply not be any relevant conditioning variable for the conditional independence of $X$ and $Y$ given $Z$ to hold. ${ }^{1}$ And if there is no common $Z$, not much can generally be said about the joint distribution of $X, Y$ from only their marginal distributions.

In this chapter, I propose an identification strategy based on sample combination that does not rely on either units or variables in common. Instead, I assume that a third sample is available, where a known linear function of $X$ and $Y$ (such as their sum, average or difference) is observed. This of course implies that $X$ and $Y$ are aggregable, which I emphasize with a change of notation. From now on, let $X_{1}$ and $X_{2}$ denote the variables whose joint distribution is of interest, and $X_{s}$ be the aggregate variable observed in a third, independent sample. My main result is to show that, when there is no bivariate sample of $X_{1}, X_{2}$, their joint distribution can be non-parametrically identified by combining the marginal distributions of $X_{1}, X_{2}$ and $X_{s}$, where $X_{s}=X_{1}+X_{2}$.

\footnotetext{
${ }^{1}$ As discussed in Ridder and Moffitt (2007), the joint cumulative distribution function (CDF) of $X, Y$ is identified without further assumptions only if either $X$ or $Y$ are fully determined by $Z$. In all other cases, identification requires additional restrictions.
} 
The data setting considered here is quite specific, but it is encountered in practice in multiple contexts. A first example is when individual and household surveys provide, for a given population, independent samples where the same variable is measured at the individual level in the one and at the household level in the other. In consumer surveys, the usual units of observation are individuals or households. In the former case, most questions relate to the respondent's own individual characteristics and behaviors. In the latter case, household aggregates are often reported (e.g., family totals ). While different household members have their marginal distributions identified by the individual level data, the household data provide information on the distribution of their sums. In such setting, the method proposed in this chapter permits to investigate intra-household questions in the absence of intra-household data. Potential applications in the context of household economics are numerous, regarding for example consumption, income, or financial assets and liabilities. In the application presented in Chapter 3, I analyse intra-household payment behaviors by combining individual-level and aggregate household payment survey data. Other data situations where unit aggregation could potentially be exploited pertain to geographical or sectorial aggregation, or to price aggregation. ${ }^{2}$

Besides unit aggregation, the method also applies to time aggregation. Given that one has measures of annual income in one data set, and monthly (or weekly) income in another data set, the joint distribution of income over time can be extracted and, for example, persistence over time can be analysed. ${ }^{3}$

Although encountered in real-data situations, the particular data setting considered in this chapter has not received much attention in the statistical or economic literature. I show that it can be exploited to recover the joint distribution of interest

\footnotetext{
${ }^{2}$ Piterbarg (2011) considers the situation where options markets provide information on the distributions of two individual underlyings (e.g. stock prices or interest rates) as well as their spread. He derives necessary and sufficient conditions for the existence of a joint distribution consistent with given marginals and the distribution of the spread.

${ }^{3}$ I am thankful to Prof. Thomas Lemieux for this suggestion of application.
} 
without making any parametric assumptions. The chapter is organized as follows. In Section 2.2, I express the problem in mathematical terms and review the related literatures. Section 2.3 provides the main identification result, while Section 2.4 discusses the implications and potential extensions of the model. In Section 2.5, estimation strategies are proposed and their consistency is shown. Finally, their small sample behavior is analysed in Section 2.6.

\subsection{Identification Problem and Related Literatures}

The identification problem considered in this chapter boils down to recovering the joint distribution of the random variables $X_{1}$ and $X_{2}$ from their respective marginal distributions and the marginal distribution of their sum $X_{s}=X_{1}+X_{2}$. Abstracting from any existence issue, I assume that the distributions of $X_{1}, X_{2}$ and $X_{s}$ emanate from an existing underlying joint distribution of $X_{1}, X_{2}$. In other words, $X_{1}, X_{2}$ and $X_{s}$ are assumed compatible.

In general, the joint distribution is only partially identified from the marginal distributions alone. The set of all possible multivariate distributions with given marginal distributions is called a Fréchet class. An early result on inequalities in bivariate Fréchet classes, dating back to Hoeffding's 1940 paper $^{4}$ and Fréchet (1951), bounds the joint $\mathrm{CDF}$ of $X_{1}, X_{2}$ as follows:

$$
\max \left(0, F_{X_{1}}\left(x_{1}\right)+F_{X_{2}}\left(x_{2}\right)-1\right) \leq F_{X_{1}, X_{2}}\left(x_{1}, x_{2}\right) \leq \min \left(F_{X_{1}}\left(x_{1}\right), F_{X_{2}}\left(x_{2}\right)\right)
$$

for all $x_{1}, x_{2}$ in $(-\infty, \infty)$. As noted in Ridder and Moffitt (2007), these bounds are often not very informative. For example, if the marginal and joint distributions are all normal, the correlation coefficient of $X_{1}$ and $X_{2}$ can take any value between -1 and 1. Dall'Aglio (1972) studies under which conditions a given bivariate Fréchet

\footnotetext{
${ }^{4}$ Originally published in German; an English translation can be found in Fisher and Sen (2012).
} 
class contains a unique distribution function. ${ }^{5}$

When the marginal distributions of $X_{1}$ and $X_{2}$ are known, the distribution of the sum $X_{s}$ provides some information on their dependence. For example, in the case of elliptical distributions - which include multivariate normal distributions - the joint distribution of $X_{1}, X_{2}$ is completely determined by their marginal distributions and the distribution of their sum. This directly follows from the fact that the dependence between two elliptical random variables is fully described by their marginal distributions and their Pearson's linear correlation coefficient; see Embrechts et al. (2002).

In general however, the joint distribution is not completely pinned-down by the auxiliary information provided by the sum. This is most easily seen in the discrete case. Let $D_{1}$ and $D_{2}$ be two discrete random variables taking natural values from 1 to $K$. Their joint distribution is discrete with $K^{2}$ mass points. The two marginal probabilities observed provide $2 K$ equality constraints, augmented with $2 K-1$ constraints from the distribution of $D_{s}=D_{1}+D_{2}\left(D_{s}=2,3, \ldots, 2 K\right)$. Three redundant constraints finally leave us with $K^{2}-4(K-1)$ degrees of freedom. The joint discrete distribution is thus exactly identified for $K=2$, but under-identified for $K>2$. Hence, in the case of categorical variables with known marginals, $D_{s}$ does not provide enough additional information to completely identify the underlying joint distribution - except when $D_{1}$ and $D_{2}$ are both binary. ${ }^{6}$

While Fréchet bounds on the joint CDF is all that can be learned from the marginals of $X_{1}$ and $X_{2}$, improved Fréchet bounds can be derived when additional

\footnotetext{
${ }^{5}$ Several strands of the statistical and econometric literature tackle the problem of identifying a joint distribution (or a related regression model) when only marginals are known. In the contingency table literature, the identification problem consists in inferring individual cell counts (or probabilities) based on observations of marginal row and column totals; see Dobra and Fienberg (2001) for a recent take on an old problem from a disclosure limitation perspective. Similarly, the ecological inference problem consists in examining the association between two binary variables across a series of 2 by 2 tables, when only the margins are observed; see Wakefield (2004).

${ }^{6}$ Here $D_{1}$ and $D_{2}$ were assumed to have the same support. In general, the number of constraints imposed by $D_{s}$ will depend on the number of distinct values that it takes (number of points in its support).
} 
information is available on the dependence between $X_{1}$ and $X_{2}$. Nelsen et al. (2001) compute improved bounds when a measure of association such as Kendall's $\tau$ or Spearman's $\rho$ is given. Tankov (2011) also considers the situation where Pearson's linear correlation coefficient is known. However, to the best of my knowledge, no closed-form solutions for improved Fréchet bounds on the CDF of two addends when the distribution of their sum is known have ever been derived in the literature.

More generally, Nelsen et al. (2004) find best-possible bounds on arbitrary sets of bivariate distribution functions with given margins. Their central result is that in order to study bounds on sets of joint distribution functions (with common margins), it is only needed to study bounds on the corresponding sets of copulas. The method of copulas offers indeed a natural framework for dealing with distributions with fixed marginals. According to Sklar's theorem, any bivariate distribution function $F_{X_{1}, X_{2}}$ can be decomposed into two components, the marginal distributions and the copula, and expressed in the form $F_{X_{1}, X_{2}}\left(x_{1}, x_{2}\right)=C\left[F_{X_{1}}\left(x_{1}\right), F_{X_{2}}\left(x_{2}\right)\right]$, where the copula $C$ is unique if $F_{X_{1}}$ and $F_{X_{2}}$ are continuous, and uniquely determined on Range $\left(F_{X_{1}}\right) \times$ Range $\left(F_{X_{2}}\right)$ otherwise; see Nelsen (2013) and Fan and Patton (2014). Applying the methodology of Nelsen et al. (2004) for the problem at stake in the present chapter would require a (copula-like) description of the set of joint distributions that match a given distribution of the sum $X_{s}=X_{1}+X_{2}$. To the best of my knowledge, such a theoretical description has still to be derived; see also Piterbarg (2011, p.5). In fact, evidence from the statistical literature on joint mixability suggests the difficulty of this task; see, e.g., Wang and Wang (2015).

Let me briefly introduce the concept of joint mixability, and explain how it relates to the identification problem at stake. A vector $\left(X_{1}, \ldots, X_{n}\right)$ is called a joint mix if $X_{1}+\ldots+X_{n}$ is almost surely a constant, and an $n$-tuple of distributions $\left(F_{X_{1}}, \ldots, F_{X_{n}}\right)$ is said to be jointly mixable if there exists a joint mix with univariate marginal distributions $F_{X_{1}}, \ldots, F_{X_{n}}$. Let $F_{-X_{s}}(x)=\operatorname{Pr}\left(-X_{s} \leq x\right)$. The question of joint 
mixability of $\left(F_{X_{1}}, F_{X_{2}}, F_{-X_{s}}\right)$ is then equivalent to the question of existence of random variables $X_{1}, X_{2}$ and $X_{s}=X_{1}+X_{2}$ such that $X_{1} \sim F_{X_{1}}, X_{2} \sim F_{X_{2}}, X_{s} \sim F_{s}$ and $X_{1}+X_{2}-X_{s}=0$. Hence, it is also equivalent to the question of existence of a joint distribution with given marginals and aggregate distribution.

A theoretical branch of the joint mixability literature provides necessary (and in some cases sufficient) conditions for the joint mixability of certain distributions; see Wang and Wang (2011), Puccetti and Wang (2015) and Wang and Wang (2015). However, explicit expressions for compliant joint distribution functions (or their copula) are acknowledged to be very hard to derive. Alternatively, the empirical strand of this literature provides algorithms to approximate them numerically; see Bernard and McLeish (2015) and citations herein. ${ }^{7}$ Yet, such algorithms merely construct individual solutions, so that the set of all solutions - as well as the bounds on this set - can only be approximated by running the algorithms a large number of times.

The statistical literature on joint mixability offers some insight into the challenge of writing down an explicit joint distribution function for random variables with fixed marginal and aggregate distributions - in general, it can only be approximated numerically. By contrast, this chapter establishes non-parametric identification of the joint distribution of $X_{1}, X_{2}$ from their respective marginal distributions and the marginal distribution of their sum. This result is obtained by using a simple nonparametric model, borrowed from Linton and Whang (2002), to model the individual series $X_{k}, k=1,2$.

Linton and Whang (2002) consider the situation where only aggregated data (group totals) are available, but the underlying individual quantities and relationships are of interest. ${ }^{8}$ This setting, where the data is not a direct measurement of

\footnotetext{
${ }^{7}$ In practice, following Rüschendorf and Uckelmann (2002), the problem is expressed as a constrained variance minimization problem.

${ }^{8}$ The vast literature dedicated to analyzing patterns of intra-household resource allocation typically operates in a similar setting, where only household totals are observed. Assumptions are then needed for identification to be achieved; see for example Vermeulen (2002) for a short introduction to the unitary and collective household models.
} 
the quantity of interest but the sum of more than one target quantity, is analyzed using so-called aggregated data models together with non-parametric deconvolution techniques; see Meister (2009). Most often, the aggregates are assumed to be the sums of independent and identically distributed random variables. But Linton and Whang (2002), interestingly, relax the independence assumption by introducing a shared component common to all addends within a same aggregate. To obtain nonparametric identification of the marginal distribution of the addends, they must however maintain the assumption of identical individual distributions both within and across groups (they also require that there are at least two different group sizes).

In the data setting considered in this chapter, independent univariate samples of the individual variables are readily available, meaning that individual marginal distributions are directly identified from the data. It is the joint distribution within groups that is the unknown quantity of interest. Generalizing the model of Linton and Whang (2002), I show that the latter is non-parametrically identified while allowing for heterogeneity both within and across groups of different size. The main model and identification result are presented in the following section.

\subsection{Model and Identification}

The main feature of the model is that dependence across variables is induced by a common component, shared by all the individual data series. In the case of two individual variables $X_{1}$ and $X_{2}$, this consists in modeling two dependent variables using three independent variables.

\subsubsection{Model}

$X_{1}$ and $X_{2}$ are each modeled as the sum of two orthogonal components, an idiosyncratic component and a common (shared) component, as follows: 


\section{Model 1.}

$$
\begin{aligned}
& X_{1 i}=V_{1 i}+V_{3 i}, \\
& X_{2 i}=V_{2 i}+V_{3 i}, \\
& X_{s i}=V_{1 i}+V_{2 i}+2 V_{3 i},
\end{aligned}
$$

where $V_{1}, V_{2}$ and $V_{3}$ are mutually independent real random variables, independent and identically distributed (iid) across $i$, and the expression for $X_{\text {si }}$ follows from $X_{s i} \equiv X_{1 i}+X_{2 i}$

Heterogeneity between $X_{1}$ and $X_{2}$ can come from different idiosyncratic terms $V_{1}$ and $V_{2}$, while dependence between the variables is induced by the common term $V_{3}$. In the next subsection I show that, in this framework, the joint distribution of $X_{1 i}, X_{2 i}$ is identifiable by combining their marginals and the distribution of the sum. The implicit constraints involved by this model as well as potential extensions are discussed in Section 2.4.

\subsubsection{Main identification result}

It is convenient, given the linearity and independence assumptions in the model under consideration, to work with characteristic functions $(\mathrm{CF})$. The $\mathrm{CF}$ of a random variable $X$ with $\operatorname{CDF} F_{X}(x)$ is defined as

$$
\begin{aligned}
\phi_{X}(t) & =\int_{-\infty}^{\infty} e^{i t x} d F_{X}(x) \\
& =E\left(e^{i t X}\right)
\end{aligned}
$$

where $i=\sqrt{-1}$. Because every bounded and measurable function is integrable with respect to any distribution function over $(-\infty, \infty)$ (Cramér, 1939), the CF of any random variable always exists. 
I briefly review here some properties of the CF that will be called upon later in this chapter, and refer the reader to Lukacs (1970) and Ushakov (1999) for more formal statements and proofs. CFs are uniformly continuous on the real line, with $\phi_{X}(0)=1$ and $\phi_{X}(-t)=\overline{\phi_{X}(t)}$ (where the bar atop $\phi_{X}(t)$ denotes its complex conjugate). One fundamental property assures that the CF completely "characterizes" its distribution function: the uniqueness theorem states that two CDFs are identical if and only if their CFs are identical. This one-to-one correspondence between a CF and a distribution function is made explicit by the inversion theorem, which will be formally stated later in the present section. A second important property, implied by the convolution theorem, is that the CF of the sum of independent random variables equals the product of their individual CFs.

The joint density of the random variables $X_{1}, X_{2}$ is uniquely determined by the joint $\mathrm{CF} \phi_{X_{1}, X_{2}}(t, u)=E\left(e^{i\left(t X_{1}+u X_{2}\right)}\right)$. The latter, under Model 1 and by the convolution theorem, can be written as

$$
\phi_{X_{1}, X_{2}}(t, u)=\phi_{V_{1}}(t) \phi_{V_{2}}(u) \phi_{V_{3}}(t+u) .
$$

The available data sets are assumed to provide the marginal distributions of $X_{1}$ and $X_{2}$, as well as the marginal distribution of the sum $X_{s}=X_{1}+X_{2}$. By the convolution theorem, Model 1 implies

$$
\begin{aligned}
& \phi_{X_{1}}(t)=\phi_{V_{1}}(t) \phi_{V_{3}}(t), \\
& \phi_{X_{2}}(t)=\phi_{V_{2}}(t) \phi_{V_{3}}(t), \text { and } \\
& \phi_{X_{s}}(t)=\phi_{V_{1}}(t) \phi_{V_{2}}(t) \phi_{V_{3}}(2 t) .
\end{aligned}
$$


By substitution, I obtain

$\phi_{X_{1}, X_{2}}(t, u)=\phi_{X_{1}}(t) \phi_{X_{2}}(u) \frac{\phi_{V_{3}}(t+u)}{\phi_{V_{3}}(t) \phi_{V_{3}}(u)}$.

While we need to identify the ratio $\Phi(t, u) \equiv \frac{\phi_{V_{3}}(t+u)}{\phi_{V_{3}}(t) \phi_{V_{3}}(u)}$ for all $t, u$ in $(-\infty, \infty)$, the data only directly identifies $\Phi(t, t)$ since

$$
\Phi(t, t)=\frac{\phi_{V_{3}}(2 t)}{\left[\phi_{V_{3}}(t)\right]^{2}}=\frac{\phi_{X_{s}}(t)}{\phi_{X_{1}}(t) \phi_{X_{2}}(t)}
$$

To find $\phi_{X_{1}, X_{2}}(t, u)$, we must therefore identify $\phi_{V_{3}}$ separately.

Lemma 1 below states that the distribution of $V_{3}$ is indeed determined, up to location, by the available data under Model 1 and some additional assumptions. ${ }^{9}$ This result is a variation of Kotlarski's lemma. Kotlarski (1967) shows that, under some conditions, the distributions of $V_{1}, V_{2}$ and $V_{3}$ in Model 1 are determined up to a change of the location by the joint distribution of $X_{1}, X_{2}$. His result has been widely applied to identify, inter alia, measurement error and panel data models. In Lemma 1, I show that it is enough to know the (marginal) distributions of $X_{1}, X_{2}$ and $X_{s}=X_{1}+X_{2}$ to obtain the same result. ${ }^{10}$

Lemma 1. Under the assumptions of Model 1, if the CFs of $X_{1}, X_{2}$ and $X_{s}=X_{1}+X_{2}$ do not vanish, then the distributions of $X_{1}, X_{2}$ and $X_{s}$ determine the distributions of $V_{1}, V_{2}, V_{3}$ up to changes of the location.

\section{Proof. See Appendix C.1.}

It is important to observe that, although the distribution of $V_{3}$ is only determined

\footnotetext{
${ }^{9}$ It can be seen immediately from Model 1 that the first moments of the $X_{k}$ s are not identified (two linearly independent equations for three unknowns), while all higher moments are identified.

${ }^{10}$ Rényi (1950) considers the problem of determining the distributions of $n$ independent random variables when the distributions of $n$ linear combinations of these random variables are known, and provides necessary and sufficient conditions for exact identification. We are exactly in his set-up, with $n=3$; however, it can be checked that the conditions for the unique determination of the distributions of $V_{1}, V_{2}$ and $V_{3}$ are not met.
} 
up to location, the ratio $\Phi(s, t)$ is uniquely determined by the available data. This leads to my main identification result presented in Theorem 1.

Theorem 1. Under the assumptions of Model 1, if the CFs of $X_{1}, X_{2}$ and $X_{s}$ do not vanish, then the distributions of $X_{1}, X_{2}$ and $X_{s}$ identify exactly the joint distribution of $X_{1}, X_{2}$.

Proof. See Appendix C.1.

\subsubsection{Regularity conditions}

If the assumptions of non-vanishing CFs is omitted, then Lemma 1 doesn't hold. One can easily see that if the CFs are allowed to take the value zero, then the equalities in (C.2) (Appendix C.1) might hold even though $\phi_{V_{k}}$ and $\phi_{U_{k}}$ are not identical for all $k$. Note also that, to avoid any division by zero, (2.11) and (2.12) also require that $\phi_{V_{3}}(t), \phi_{X_{1}}(t)$ and $\phi_{X_{2}}(t)$ be nonzero for $-\infty<t<\infty$. This further implies that

$\phi_{V_{1}}(t), \phi_{V_{2}}(t)$ (from Equations (2.8) and (2.9)) and $\phi_{X_{s}}(t)$ (from Equation (2.10)) are also non-vanishing. Hence, the conditions stated in Lemma 1 and Theorem 1 are equivalent to avoiding division by zero.

It is usual, in the literature on density deconvolution and errors-in-variables regression to assume that the $\mathrm{CF}$ of the measurement error (that appears in the denominator of the CF to be Fourier-inversed) is everywhere non-vanishing. This assumption is satisfied by most common continuous distributions as well as many discrete distributions. As discussed in D'Haultfoeuille (2011), the only common continuous distributions that fail to satisfy it are the uniform and triangular distributions. However, all finite (i.e., left and right bounded) distribution functions have CFs with infinitely many zeros (Lukacs, 1970).

Kotlarski (1967) mentions that his assumption of non-vanishing of the CF (in his case, it is the joint $\mathrm{CF}$ of $X_{1}, X_{2}$ ) can be replaced by the assumption that all $V_{k}$ 
components have analytic CFs. ${ }^{11}$ This directly applies to my case too. Following Evdokimov and White (2012) it can further be shown that Lemma 1 also holds when only one of the $V_{k}$ s has an analytic CF. The reasoning goes as follows: Because any $\mathrm{CF}$ is non-vanishing in a region around zero (as it is continuous and takes value 1 at 0), the result of Lemma 1 holds in this region for any CF. Analyticity of a CF then permits to spread its identification on the whole real line. Given that the CF of one $V_{k}$ is identified on $\mathbb{R}$ - say $\phi_{V_{3}}(t)$ - then the remaining CFs can be recovered from (2.8) and (2.9): an analytic CF can only have isolated zeros, so that $\phi_{V_{1}}(t)=\phi_{X_{1}}(t) / \phi_{V_{3}}(t)$ and $\phi_{V_{2}}(t)=\phi_{X_{2}}(t) / \phi_{V_{3}}(t)$ for all points $t \in \mathbb{R}$ except for the isolated zeros of $\phi_{V_{3}}$. Finally, by continuity, $\phi_{V_{1}}(t)$ and $\phi_{V_{2}}(t)$ are identified on the whole real line. My identification result might also hold under alternative conditions, as discussed in Evdokimov and White (2012).

\subsection{Restrictions and Extensions of the Model}

\subsubsection{Testable restrictions}

Model 1 has several important implications for the moments of the joint distribution of $X_{1}, X_{2}$. First and foremost, we have that

$$
\operatorname{Cov}\left(X_{1}, X_{2}\right)=\operatorname{Var}\left(V_{3}\right) \geq 0
$$

Thus, as modeled in Model $1, X_{1}$ and $X_{2}$ are necessarily positively correlated. Other restrictions imposed by the model are the following:

$$
\begin{aligned}
& \operatorname{Cov}\left(X_{1}, X_{2}\right)=\operatorname{Var}\left(V_{3}\right) \leq \operatorname{Var}\left(X_{1}\right) \\
& \operatorname{Cov}\left(X_{1}, X_{2}\right)=\operatorname{Var}\left(V_{3}\right) \leq \operatorname{Var}\left(X_{2}\right)
\end{aligned}
$$

\footnotetext{
${ }^{11}$ Analytic CFs and their properties are thoroughly discussed in Lukacs (1970).
} 
Relations given in (2.13) and (2.14) must necessarily hold in the data for Model 1 to be valid (but this is of course not sufficient). Importantly, these restrictions can be tested in the data. ${ }^{12}$

I propose now variants that could be used when Model 1 seems inappropriate for the data at hand, and discuss the conditions under which identification of the joint distribution of interest is guaranteed.

\subsubsection{Extensions of the baseline model}

\subsubsection{Variations in the decomposition of $X_{1}$ and $X_{2}$}

The baseline model can be extended to allow the common component $V_{3}$ to have asymmetric effects on $X_{1}$ and $X_{2}$. This can be done by introducing scale parameters, as follows:

\section{Model 2.}

$$
\begin{aligned}
& X_{1 i}=V_{1 i}+\rho_{1} V_{3 i}, \\
& X_{2 i}=V_{2 i}+\rho_{2} V_{3 i}, \\
& X_{s i}=V_{1 i}+V_{2 i}+\left(\rho_{1}+\rho_{2}\right) V_{3 i},
\end{aligned}
$$

where $V_{1}, V_{2}$ and $V_{3}$ are mutually independent real random variables, iid across $i$, and $\rho_{1}, \rho_{2}$ are real constants.

In Model 2, the sign of $\operatorname{Cov}\left(X_{1}, X_{2}\right)$ depends on the values of the scale parameters. If $\rho_{1}=\rho_{2}=1$, this model boils down to Model 1 with positive correlation. If $\rho_{1} \rho_{2}<0$, the correlation is negative. For any values of $\rho_{1}$ and $\rho_{2}$ such that $\rho_{1}+\rho_{2} \neq 0$, the general identification result for $\phi_{X_{1}, X_{2}}$ holds.

\footnotetext{
${ }^{12}$ Higher order moments of the joint distribution are also impacted by my modeling assumptions, but these restrictions are less clear-cut.
} 
Note however that if $\rho_{1}$ and $\rho_{2}$ are such that $\rho_{1}+\rho_{2}=0$ (so that the term $V_{3}$ does not appear in $X_{s}$ ), then $\phi_{V_{3}}$ is not always identified. Equation (2.12) in such a case becomes

$$
\frac{\phi_{X_{s}}(t)}{\phi_{X_{1}}(t) \phi_{X_{2}}(t)}=\frac{1}{\phi_{V_{3}}(t) \phi_{V_{3}}(-t)} .
$$

Since $\phi_{V_{3}}(-t)=\overline{\phi_{V_{3}}(t)}$, we have that $\phi_{V_{3}}(t) \phi_{V_{3}}(-t)=\left|\phi_{V_{3}}(t)\right|^{2}$. Hence, in general, Equation (2.18) identifies only the modulus of $\phi_{V_{3}}$. However, under the assumption that $V_{3}$ is symmetric around zero, $\phi_{V_{3}}$ is real and even (i.e. $\phi_{V_{3}}(-t)=\phi_{V_{3}}(t)$ ) and is identified by

$$
\frac{\phi_{X_{s}}(t)}{\phi_{X_{1}}(t) \phi_{X_{2}}(t)}=\frac{1}{\left[\phi_{V_{3}}(t)\right]^{2}}
$$

Symmetry assumptions can further allow using a second type of extension of Model 1, where $X_{1}$ and $X_{2}$ are modeled using four mutually independent components instead of three. Consider for instance the following model:

\section{Model 3.}

$$
\begin{aligned}
& X_{1 i}=V_{1 i}+V_{3 i}+V_{4 i}, \\
& X_{2 i}=V_{2 i}+V_{3 i}-V_{4 i}, \\
& X_{s i}=V_{1 i}+V_{2 i}+2 V_{3 i},
\end{aligned}
$$

where $V_{1}, V_{2}, V_{3}$ and $V_{4}$ are mutually independent real random variables, iid across $i$.

It can be shown that, if $V_{4}$ is symmetrically distributed, the CFs of the four $V_{k}$ components are identified, at least up to location, by the distributions of $X_{1}, X_{2}$ and $X_{s}$. 


\subsubsection{Variations in the definition of $X_{s}$}

The decomposition of $X_{1}$ and $X_{2}$ into mutually independent components can be seen merely as a device to model two non-independent random variables. ${ }^{13}$ However, one might also be preoccupied by the interpretability of the model and its different components. For example, if $X_{1}$ and $X_{2}$ measure the expenses of two individuals within a couple, in Model 2 with $\rho_{1}=1-\rho_{2}, V_{3}$ could be interpreted as total expenses on common goods - which can be shared asymmetrically across partners. However, depending on the application, the interpretation of the common shock $V_{3}$ can be complicated by the "double-counting" of $V_{3}$ in $X_{s}=X_{1}+X_{2}$. For example, if $X$ measures asset holdings, a decomposition of individual holdings into personal $\left(V_{1}\right.$ and $V_{2}$ ) and joint $\left(V_{3}\right)$ holdings using Model 1 would lead to household totals of the form $\tilde{X}_{s}=V_{1}+V_{2}+V_{3}$. Such a variant in the definition of the aggregate variable does not jeopardize our identification result on the joint distribution of interest.

\subsubsection{Extensions with more than two individual random vari- ables}

A straightforward extension of my identification result to the case of three individual random variables $X_{1}, X_{2}$ and $X_{3}$ is obtained under the following model:

\section{Model 4.}

$$
\begin{aligned}
& X_{1 i}=V_{1 i}+V_{4 i}, \\
& X_{2 i}=V_{2 i}+V_{4 i}, \\
& X_{3 i}=V_{3 i}+V_{4 i},
\end{aligned}
$$

\footnotetext{
${ }^{13}$ As a matter of fact, "trivariate reduction" (also called the "variables in common" method) is a popular and old technique used for constructing dependent variables; see, e.g., Sarabia Alzaga and Gómez Déniz (2008) and Balakrishnan and Lai (2009).
} 
where $V_{1}, V_{2}, V_{3}$ and $V_{4}$ are mutually independent real random variables, iid across $i$.

It can be shown that the $\mathrm{CF}$ of the common component $V_{4}$ is identified up to location by the marginal distributions of the $X_{k}$ 's and the distribution of the sum $X_{s}=X_{1}+X_{2}+X_{3}$. Note indeed that the linearity and independence assumptions give

$$
\frac{\phi_{X_{s}}(t)}{\phi_{X_{1}}(t) \phi_{X_{2}}(t) \phi_{X_{3}}(t)}=\frac{\phi_{V_{4}}(3 t)}{\left[\phi_{V_{4}}(t)\right]^{3}} .
$$

From there, identification can be proved along the lines of the proof of Lemma 1.

In Model 4 , the three $X_{k}$ variables all share one common component $V_{4}$, which imposes for example that the covariance of $X_{1}, X_{2}$ is the same as that of $X_{1}, X_{3}$ and $X_{2}, X_{3}$. A much more flexible alternative is:

\section{Model 5.}

$$
\begin{aligned}
& X_{1 i}=V_{1 i}+V_{12 i}+V_{13 i}+V_{123 i}, \\
& X_{2 i}=V_{2 i}+V_{12 i}+V_{23 i}+V_{123 i}, \\
& X_{3 i}=V_{3 i}+V_{13 i}+V_{23 i}+V_{123 i},
\end{aligned}
$$

where $V_{1}, V_{2}, V_{3}, V_{12}, V_{13}, V_{23}$ and $V_{123}$ are mutually independent real random variables, iid across $i$.

To identify the distributions of the $7 V_{k}$ components in Model 5 , it is not enough to know the marginal distributions of the three $X_{k}$ variables and the total sum $X_{1}+$ $X_{2}+X_{3}$. If, however, the distributions of the three partial sums $X_{1}+X_{2}, X_{1}+X_{3}$

and $X_{2}+X_{3}$ are also available, then it can be shown that the distributions of the $V_{k}$ components are identified up to location. A formal proof would again go along the lines of that of Lemma 1. Note for instance that the linearity and independence 
assumptions of Model 5 give

$$
\frac{\phi_{X_{1}+X_{2}+X_{3}}(t) \phi_{X_{1}}(t) \phi_{X_{2}}(t) \phi_{X_{3}}(t)}{\phi_{X_{1}+X_{2}}(t) \phi_{X_{1}+X_{3}}(t) \phi_{X_{2}+X_{3}}(t)}=\frac{\left[\phi_{V_{123}}(t)\right]^{3} \phi_{V_{123}}(3 t)}{\left[\phi_{V_{123}}(2 t)\right]^{3}},
$$

which identifies $\phi_{V_{123}}(t)$ up to a multiplicative term of the form $e^{b t}$, where $b$ is a real number.

\subsubsection{Extension with overlapping samples and measurement error}

Until now I have assumed that the data samples available are independent of each other. If units in common across samples provide the joint distribution of $X_{1}, X_{s}$ or $X_{2}, X_{s}$, then the joint distribution of $X_{1}, X_{2}$ is directly identified. ${ }^{14}$ More interestingly, such an overlap can allow us to identify the joint distribution of $X_{1}, X_{2}$ in a context of measurement error, when one of the variables $X_{k}, k=1,2, s$ is observed with error.

\section{Model 6.}

$$
\begin{aligned}
& X_{1 i}=V_{1 i}+V_{3 i}, \\
& X_{2 i}=V_{2 i}+V_{3 i}, \\
& \widetilde{X}_{s i}=V_{1 i}+V_{2 i}+2 V_{3 i}+\epsilon_{i},
\end{aligned}
$$

where $V_{1}, V_{2}, V_{3}$ and $\epsilon$ are mutually independent real random variables, iid across $i$.

In this model, the observed variable $\widetilde{X}_{s}$ is measured with an additive error $\epsilon$ that is assumed independent from the true latent variable $X_{s}=X_{1}+X_{2}$. In a setting where, in addition from observing the marginal distributions of $X_{1}, X_{2}$ and $\widetilde{X}_{s}$, some overlap across samples providing the joint distribution of $X_{1}, X_{s}$ or $X_{2}, X_{s}$ can be exploited

\footnotetext{
${ }^{14}$ Using characteristic functions, note that $\phi_{X_{1}, X_{s}}(t, u)=E\left(e^{i\left(t X_{1}+u\left(X_{1}+X_{2}\right)\right)}\right)=$ $E\left(e^{i\left((t+u) X_{1}+u X_{2}\right)}\right)=\phi_{X_{1}, X_{2}}(t+u, u)$.
} 
to identify the joint distribution of $X_{1}, X_{2}$. Note that the linearity and independence assumptions of Model 6 give

$$
\frac{\phi_{\widetilde{X}_{s}, X_{1}}(t, t) \phi_{X_{1}}(t)}{\phi_{\widetilde{X}_{s}}(t) \phi_{X_{1}}(2 t)}=\frac{\phi_{V_{3}}(3 t) \phi_{V_{3}}(t)}{\left[\phi_{V_{3}}(2 t)\right]^{2}},
$$

which identifies $\phi_{V_{3}}(t)$ up to a multiplicative term of the form $e^{b t}$, where $b$ is a real number.

\subsubsection{Conditional analysis}

We might be interested in the joint distribution of $X_{1}, X_{2}$ after conditioning on observables. Let $Z$ be a vector of variables that are observed in all samples. That is, let us assume for now that the available data consists in three samples $\left\{X_{1 i}, Z_{i}\right\}_{i=1}^{n_{1}}$ , $\left\{X_{2 i}, Z_{i}\right\}_{i=1}^{n_{2}}$ and $\left\{X_{s i}, Z_{i}\right\}_{i=1}^{n_{s}} \cdot{ }^{15}$ One approach for conditional analysis is to work with conditional CFs. The conditional CF of $X$ given $Z$ is defined as

$$
\begin{aligned}
\phi_{X \mid Z}(t \mid z) & =\int_{-\infty}^{\infty} e^{i t x} d F_{X \mid Z}(x \mid z) \\
& =E\left(e^{i t X} \mid Z=z\right),
\end{aligned}
$$

Evdokimov (2010) first considered the use of conditional deconvolution procedures, in the setting of a non-parametric panel data model. In the context of a measurement error model, Firpo et al. (2015) recover a conditional density from its conditional CF. Working with conditional CFs, our main identification result hold: the conditional joint $\mathrm{CF}$ of $X_{1}$ and $X_{2}$ is identified under Model 1 (and the various extensions discussed in the beginning of this section). However, in a non-parametric framework, such approach requires very large sample sizes. Consider that, when the conditioning variables are discrete, conditional CFs can be obtained by splitting the

\footnotetext{
${ }^{15}$ In the context of consumer surveys, for example, household income is usually collected in both individual and household surveys.
} 
samples according to the values of $Z$.

Another approach for conditional analysis would be to work with regression residuals instead of raw variables. To apply Theorem 1, it is necessary that the residuals obtained from the regressions of the individual data series $X_{1}$ and $X_{2}$ sum up to the residuals obtained from the regression of $X_{s}$. I will discuss two situations when this is the case. Suppose the true regression models for $X_{1}$ and $X_{2}$ are

$$
\begin{aligned}
& X_{1}=Z \beta_{1}+\epsilon_{1}, \\
& X_{2}=Z \beta_{2}+\epsilon_{2} .
\end{aligned}
$$

If $X_{s} \equiv X_{1}+X_{2}$, the true aggregate model is

$$
X_{s}=Z\left(\beta_{1}+\beta_{2}\right)+\epsilon_{1}+\epsilon_{2} .
$$

Hence the error term of the regression of $X_{s}$ on $Z, \epsilon_{s}$, is such that $\epsilon_{s}=\epsilon_{1}+\epsilon_{2}$. Under the condition that the $\beta \mathrm{s}$ can be unbiasedly estimated in each equations, $\hat{\epsilon}_{s}=\hat{\epsilon}_{1}+\hat{\epsilon}_{2}$ holds for fitted residuals. Thus, Model 1's decomposition can be applied to the residuals $\hat{\epsilon}_{1}$ and $\hat{\epsilon}_{2}$, and their joint distribution can be recovered from $\hat{\epsilon}_{1}, \hat{\epsilon}_{2}$ and $\hat{\epsilon}_{s}$.

Let us now consider a different situation where the available data consists in three samples $\left\{X_{1 i}, Z_{1 i}\right\}_{i=1}^{n_{1}},\left\{X_{2 i}, Z_{2 i}\right\}_{i=1}^{n_{2}}$ and $\left\{X_{s i}, Z_{1 i}, Z_{2 i}\right\}_{i=1}^{n_{s}}{ }^{16}$ To apply Theorem 1, it is again necessary that the residuals obtained from the regressions of $X_{1}\left(\right.$ on $Z_{1}$ ) and $X_{2}$ (on $Z_{2}$ ) sum up to the residuals obtained from the regression of $X_{s}$ (on $Z_{1}$ and $Z_{2}$ ). If the true regression models for the individual series are

$$
\begin{aligned}
& X_{1}=Z_{1} \beta_{1}+\epsilon_{1}, \\
& X_{2}=Z_{2} \beta_{2}+\epsilon_{2},
\end{aligned}
$$

\footnotetext{
${ }^{16}$ In the context of consumer surveys, for example, individual surveys ask for the age of the respondent while household surveys ask for the age of every individual within the household.
} 
and if $X_{s} \equiv X_{1}+X_{2}$, then the true aggregate model is

$$
X_{s}=Z_{1} \beta_{1}+Z_{2} \beta_{2}+\epsilon_{1}+\epsilon_{2}
$$

Hence the error term of the regression of $X_{s}$ on both $Z_{1}$ and $Z_{2}, \epsilon_{s}$, is such that $\epsilon_{s}=\epsilon_{1}+\epsilon_{2}$, and my identification strategy is applicable on the residuals. Note that if only $Z_{s}=Z_{1}+Z_{2}$ is observed in the aggregate sample, then the residuals of the regression of $X_{s}$ on $Z_{s}$ cannot be combined to that of the individual regressions to recover the joint distribution.

\subsection{Estimation}

This section discusses non-parametric methods for estimating the joint density of $X_{1}$, $X_{2}$ based on the main identification result of Section 2.3. I propose an estimation

process in two steps : 1) Estimate $\left.\phi_{V_{3}} ; 2\right)$ Derive $\widehat{f}_{X_{1}, X_{2}}$ from $\widehat{\phi}_{X_{1}, X_{2}}$.

\subsubsection{Estimating $\phi_{V_{3}}$}

The CF of $X_{k}, k=1,2, s$, is consistently estimated by the empirical characteristic function $(\mathrm{ECF})$

$$
\widehat{\phi}_{X_{k}}(t)=\frac{1}{n_{k}} \sum_{j=1}^{n_{k}} e^{i t X_{k j}},
$$

with $n_{k}$ the size of the sample where $X_{k}$ is observed.

Several procedures can be applied to estimate the $\mathrm{CF}$ of $V_{3}$, depending on the assumptions made on $\phi_{V_{3}}$. Observe that if $\phi_{V_{3}}(t)$ is non-vanishing, we can write by 
Equation (2.12), ${ }^{17}$

$$
\ln \Phi(t, t)=\ln \phi_{V_{3}}(2 t)-2 \ln \phi_{V_{3}}(t)
$$

Let $\widehat{\Phi}(t, t)=\frac{\widehat{\phi}_{X_{s}}(t)}{\widehat{\phi}_{X_{1}}(t) \widehat{\phi}_{X_{2}}(t)}$. In the case where $V_{3}$ is assumed to be symmetrically distributed around zero, $\phi_{V_{3}}(t)$ is real-valued. Then, as suggested in Linton and Whang (2002), a consistent estimator of $\kappa_{3}(t)=\ln \phi_{V_{3}}(t)$ is obtained by carrying out the non-parametric regression of $\ln \widehat{\Phi}(t, t)$ on $t$ in a way that imposes the structure of the right-hand side of (2.42). For example, using a power series approximation, we get

$$
\widehat{\kappa}_{3}(t)=\sum_{j=2}^{J_{n}} \hat{a}_{j} t^{j}
$$

where $J_{n} \rightarrow \infty$ as $n \rightarrow \infty$ and the $a_{j}$ coefficient estimates, $j=2, \ldots, J_{n}$, are found by applying ordinary least squares to

$$
\ln \widehat{\Phi}(t, t)=\sum_{j=2}^{J n} 2 a_{j}\left(2^{j-1}-1\right) t^{j}
$$

Note that the summation in (2.43) starts at $j=2 . a_{0}=0$ follows from $\phi_{V_{3}}(0)=1$ (for any $\mathrm{CF}$ ). A parameter $a_{1}$ would not be identified from (2.44). However, the constraint $a_{1}=0$ imposes $E\left(V_{3}\right)=0$ without any consequence for our estimation of $\Phi(s, t)$ and $\phi_{X_{1}, X_{2}} \cdot{ }^{18}$

The more general case where $\phi_{V_{3}}$ is not assumed to be symmetric (but still assumed non-vanishing) can be handled as proposed in Horowitz and Markatou (1996). $\phi_{V_{3}}$ is then a complex-valued function that can be written as $\phi_{V_{3}}(t)=\left|\phi_{V_{3}}(t)\right| e^{i \nu(t)}$. By the

\footnotetext{
${ }^{17}$ Note that the right-hand side ratio in Equation (2.12) is defined only for $t$ such that $\phi_{X_{1}}(t)$ and $\phi_{X_{2}}(t)$ are not zero. This is the case if and only if $\phi_{V_{k}}(t) \neq 0$ for $k=1,2,3$.

${ }^{18}$ This directly relates to the fact that $\phi_{V_{3}}$ is identified up to location only. The mean of a random variable relates to the first derivative of its CF by the formula $E(X)=-i \phi_{X}^{\prime}(0)$, but note that $\kappa_{3}^{\prime}(0)=a_{1}$ is not identified iff $a_{1}$ is not identified.
} 
mathematical properties of the modulus of a complex number, Equation (2.12) gives

$$
\begin{aligned}
\Phi(t, t) & =\frac{\left|\phi_{V_{3}}(2 t)\right| e^{i \nu(2 t)}}{\left|\phi_{V_{3}}(t)\right|^{2} e^{i 2 \nu(t)}} \\
& =\left|\frac{\phi_{V_{3}}(2 t)}{\phi_{V_{3}}(t)^{2}}\right| \frac{e^{i \nu(2 t)}}{e^{i 2 \nu(t)}} \\
& =|\Phi(t, t)| e^{i(\nu(2 t)-2 \nu(t))}
\end{aligned}
$$

Note that $\left|\phi_{V_{3}}(t)\right|$, which is always real and positive, can be consistently estimated from $|\Phi(t, t)|=\frac{\left|\phi_{V_{3}}(2 t)\right|}{\left|\phi_{V_{3}}(t)\right|^{2}}$ following the procedure just described. Finally, from (2.45) we have

$$
\operatorname{Im}\left\{\ln \frac{\Phi(t, t)}{|\Phi(t, t)|}\right\}=\nu(2 t)-2 \nu(t)
$$

where Im denotes the imaginary part of a complex number. A consistent estimator of $\nu(t)$ can then be obtained by carrying out the non-parametric regression of $\operatorname{Im}\left\{\ln \frac{\hat{\Phi}(t, t)}{|\hat{\Phi}(t, t)|}\right\}$ on $t$ in a way that imposes the structure of the right-hand side of (2.46).

An alternative estimation method can be found in Meister et al. (2010). To exactly fit their framework, we rescale $X_{s}$ and define $\bar{X}=\frac{1}{2}\left(X_{1}+X_{2}\right)$. Under Model 1 ,

$$
\bar{X}=\frac{1}{2}\left(V_{1}+V_{2}\right)+V_{3}
$$


Let

$$
\begin{aligned}
\bar{\Phi}(t) & =\prod_{k=0}^{K}\left[\phi_{\bar{X}}\left(t / 2^{k}\right)\right]^{2^{k}}, \\
\Phi_{1}(t) & =\prod_{k=0}^{K}\left[\phi_{X_{1}}\left(t / 2^{k+1}\right)\right]^{2^{k}}, \\
\Phi_{2}(t) & =\prod_{k=0}^{K}\left[\phi_{X_{2}}\left(t / 2^{k+1}\right)\right]^{2^{k}} .
\end{aligned}
$$

Some algebra provides

$$
\begin{aligned}
\frac{\bar{\Phi}(t)}{\Phi_{1}(t) \Phi_{2}(t)} & =\prod_{k=0}^{K}\left(\frac{\phi_{\bar{X}}\left(t / 2^{k}\right)}{\phi_{X_{1}}\left(t / 2^{k+1}\right) \phi_{X_{2}}\left(t / 2^{k+1}\right)}\right)^{2^{k}} \\
& =\frac{\phi_{V_{3}}(t)}{\left[\phi_{V_{3}}\left(t / 2^{K+1}\right)\right]^{2^{K+1}}}
\end{aligned}
$$

after simplification of the telescopic product. Mild regularity assumptions on $V_{3}$ ensure that $\lim _{k \rightarrow \infty}\left[\phi_{V_{3}}\left(t / 2^{k}\right)\right]^{2^{k}}=1$; Meister et al. (see 2010, Assumption 2.1). (2.50) thus implies that $\frac{\bar{\Phi}(t)}{\Phi_{1}(t) \Phi_{2}(t)}$ is an approximation of $\phi_{V_{3}}(t)$. Under the condition that the denominator $D(t)=\prod_{k=0}^{K}\left[\phi_{X_{1}}\left(t / 2^{k+1}\right) \phi_{X_{2}}\left(t / 2^{k+1}\right)\right]^{2^{k}}$ never vanishes, a consistent estimator for $\phi_{V_{3}}(t)$ is obtained by substituting the ECFs for the CFs of $\bar{X}, X_{1}$ and $X_{2}$ in the right-hand side expression of Equation (2.49):

$$
\widehat{\phi}_{V_{3}}(t)=\frac{\prod_{k=0}^{K}\left[\widehat{\phi}_{\bar{X}}\left(t / 2^{k}\right)\right]^{2^{k}}}{\prod_{k=0}^{K}\left[\widehat{\phi}_{X_{1}}\left(t / 2^{k+1}\right) \widehat{\phi}_{X_{2}}\left(t / 2^{k+1}\right)\right]^{2^{k}}} .
$$

Note that as $K \rightarrow \infty$, the numerator in (2.49) equals zero if the denominator equals zero. By defining $\frac{0}{0}=0$, the $\phi_{V_{k}}(t)$ 's can be allowed to have countably many isolated zeros while ensuring that the ratio $\frac{\bar{\Phi}(t)}{\Phi_{1}(t) \Phi_{2}(t)}$ is always well defined . 


\subsubsection{Estimating $f_{X_{1}, X_{2}}$}

When the $\mathrm{CF} \phi_{X}(t)$ of a random variable $X$ is absolutely integrable, the Fourier inversion theorem defines the density function $f_{X}(x)$ as

$$
f_{X}(x)=\frac{1}{2 \pi} \int_{-\infty}^{\infty} e^{-i t x} \phi_{X}(t) d t .
$$

Similarly, the joint density function $f_{X_{1}, X_{2}}$ is recoverable from the joint CF by a two-dimensional Fourier inversion, as follows:

$$
\begin{aligned}
f_{X_{1}, X_{2}}\left(x_{1}, x_{2}\right) & =\frac{1}{(2 \pi)^{2}} \int_{-\infty}^{\infty} \int_{-\infty}^{\infty} e^{-i\left(t x_{1}+u x_{2}\right)} \phi_{X_{1}, X_{2}}(t, u) d t d u \\
& =\frac{1}{(2 \pi)^{2}} \int_{-\infty}^{\infty} \int_{-\infty}^{\infty} e^{-i\left(t x_{1}+u x_{2}\right)} \phi_{X_{1}}(t) \phi_{X_{2}}(u) \frac{\phi_{V_{3}}(t+u)}{\phi_{V_{3}}(t) \phi_{V_{3}}(u)} d t d u
\end{aligned}
$$

where (2.54) is obtained by substituting Equation (2.11) into (2.53).

A naive approach to construct an estimator for the joint density of $X_{1}, X_{2}$ from (2.54) would consist in replacing all the CFs by consistent empirical counterparts: the ECFs of $X_{1}$ and $X_{2}$ and a consistent estimate of $\phi_{V_{3}}$. However, such plug-in estimator doesn't work in general, because the resulting double integral can diverge. In brief, the estimation of $f_{X_{1}, X_{2}}$ in $(2.54)$ is an ill-posed inverse problem; see Horowitz (2014). ${ }^{19}$ The mapping from $f_{X_{1}, X_{2}}$ to $\phi_{X_{1}, X_{2}}$ is continuous but the inverse mapping (2.54) is not, because the integrand may be unbounded as $|t|$ or $|u|$ become large. This has for consequence that small differences between the true $\mathrm{CF} \phi_{X_{1}, X_{2}}$ and its estimate can induce large differences between the true density $f_{X_{1}, X_{2}}$ and its estimate.

As summarized in Delaigle (2014b), there are two main problems with the em-

\footnotetext{
19 "An estimation problem is called ill posed if the identifying mapping is discontinuous in a way that prevents consistent estimation of the parameter of interest by replacing the population distribution of the data with a consistent sample analog. The problem is called an ill-posed inverse problem if the discontinuous identifying mapping is obtained by inverting another mapping that is continuous." (Horowitz, 2014)
} 
pirical version of the integrand in (2.54). First, the ECF $\widehat{\phi}_{X}(t)$ is a poor estimator of the corresponding CF $\phi_{X}(t)$ for large $|t|$ - in other words, $\widehat{\phi}_{X}(t)$ is inaccurate in its tails. Second, the ECFs in the numerator are multiplied by $\frac{1}{\phi_{V_{3}}(t) \phi_{V_{3}}(u)}$, which tends in general to infinity as $|t|$ or $|u|$ go to infinity; the faster $\phi_{V_{3}}(t)$ tends to zero as $|t| \longrightarrow \infty$, the more problematic this is. This second problem would exist if the denominator in (2.54) was known, but it is exacerbated when the denominator is estimated from the data. The resulting estimates of the ratio corresponding to $\phi_{X_{1}, X_{2}}$ would be very unreliable at high frequencies $t$ and $u$, leading to wild fluctuations in the estimate of $f_{X_{1}, X_{2}}$.

To solve an ill-posed problem, some regularization is needed. It consists in removing the discontinuity of the discontinuous (inverse) mapping, so that estimation can be carried out by replacing unknown population parameters with consistent sample analogs (Horowitz, 2014). Different regularization techniques have been proposed in the non-parametric deconvolution literature. The two principal methods are ridging the integrand, see Hall and Meister (2007), and introducing a damping factor to the integrand. Multiplying by a damping factor can temper the estimator of $\phi_{X_{1}, X_{2}}$ for large frequencies, where it is most unreliable. By far the most popular approach is the deconvoluting kernel density estimator introduced by Carroll and Hall (1988) and Stefanski and Carroll (1990). The method adopted here is a special case of the deconvoluting kernel approach, when a uniform kernel is used; see Diggle and Hall (1993). ${ }^{20}$ Let $\widehat{\phi}_{V_{3}}(t)$ denote a consistent estimator of $\phi_{V_{3}}(t)$. Using truncated integrals, I propose the following estimator for $f_{X_{1}, X_{2}}\left(x_{1}, x_{2}\right)$,

$$
\widehat{f}_{X_{1}, X_{2}}\left(x_{1}, x_{2}\right)=\operatorname{Re} \frac{1}{(2 \pi)^{2}} \int_{-T_{n}}^{T_{n}} \int_{-U_{n}}^{U_{n}} e^{-i\left(t x_{1}+u x_{2}\right)} \widehat{\phi}_{X_{1}}(t) \widehat{\phi}_{X_{2}}(u) \frac{\widehat{\phi}_{V_{3}}(t+u)}{\widehat{\phi}_{V_{3}}(t) \widehat{\phi}_{V_{3}}(u)} d t d u
$$

\footnotetext{
${ }^{20}$ Many papers also combine multiple regularization techniques: kernel and truncated Fourier inversion in Liu and Taylor (1989), kernel and ridge in Delaigle et al. (2008) and Meister (2009), among others.
} 
where $T_{n}$ and $U_{n} \rightarrow \infty$ at rates to be specified in the next subsection, and where Re denotes the real part of a complex number. The real part of the right-hand side function is taken to ensure that the estimated density resulting from the truncated integration is real. ${ }^{21}$

Next I establish the rate of convergence of the non-parametric Fourier inversion estimator given in Equation (2.55).

\subsubsection{Asymptotic properties}

Under some regularity conditions, the procedures to estimate $\phi_{V_{3}}$ discussed in Section 2.5.1 can be expected to be consistent at the usual rate of convergence of nonparametric methods. For simplicity, since several estimation approaches exist, we assume in the sequel a uniform rate of convergence for the estimator $\widehat{\phi}_{V_{3}}(t)$. This is expressed in Assumption 1.

Assumption 1. There exists an estimator $\widehat{\phi}_{V_{3}}(t)$ such that

$$
\sup _{t \in \mathbb{R}}\left|\widehat{\phi_{V_{3}}}(t)-\phi_{V_{3}}(t)\right|=o\left(\beta_{n}\right) \quad \text { a.s. }
$$

with $\beta_{n}=n^{-\lambda / 2}$ for $0<\lambda \leq 1$.

Under Assumption 1, the rate of convergence of the non-parametric density estimator $(2.55)$ of $f_{X_{1}, X_{2}}\left(x_{1}, x_{2}\right)$ is given by the following theorem.

Theorem 2. Let $\phi_{X_{1}, X_{2}}(t, u)$ be absolutely integrable and let $\phi_{V_{3}}(t) \neq 0$ for all $t$. Let $T_{n}=o\left(\left(\frac{n}{\log n}\right)^{\gamma}\right)$ and $U_{n}=o\left(\left(\frac{n}{\log n}\right)^{\gamma}\right)$ with $0<\gamma<\frac{1}{2}$. Define $K_{3}(t)=$ $\inf _{|s| \leq t}\left|\phi_{V_{3}}(s)\right|$ and let $\theta_{n}=K_{3}\left(T_{n}\right)$ and $\vartheta_{n}=K_{3}\left(U_{n}\right)$. Then, under Assumption 1,

$$
\sup _{\left(x_{1}, x_{2}\right) \epsilon \chi_{1} \times \chi_{2}}\left|\widehat{f}_{X_{1}, X_{2}}\left(x_{1}, x_{2}\right)-f_{X_{1}, X_{2}}\left(x_{1}, x_{2}\right)\right|=o\left(\frac{\alpha_{n} T_{n} U_{n}}{\theta_{n} \vartheta_{n}}\right)+o\left(\frac{\beta_{n} T_{n} U_{n}}{\theta_{n} \vartheta_{n}}\right) \quad \text { a.s. }
$$

\footnotetext{
${ }^{21}$ Further, because $\widehat{f}_{X_{1}, X_{2}}\left(x_{1}, x_{2}\right)$ could be negative, $\widetilde{f}_{X_{1}, X_{2}}\left(x_{1}, x_{2}\right)=\max \left\{\widehat{f}_{X_{1}, X_{2}}\left(x_{1}, x_{2}\right), 0\right\}$ can be taken and rescaled so that it integrates to 1 , as discussed in Delaigle (2014a).
} 
with $\alpha_{n}=o(1), \frac{(\log n / n)^{\frac{1}{2}-\gamma}}{\alpha_{n}}=O(1), \frac{\beta_{n}}{\theta_{n}}=o(1)$ and $\frac{\beta_{n}}{\vartheta_{n}}=o(1)$.

Proof. See Appendix C.2.

\subsection{Monte-Carlo Simulations}

In this section, I present the finite-sample behavior of the density estimators proposed in Section 2.5. The estimation procedure's sensitivity to the truncation parameters is assessed, and a data-driven selection procedure for the truncation parameters $T$ and $U$ is suggested and tested. Finally, practical implementation is discussed.

\subsubsection{Design}

The three series $X_{1}, X_{2}$ and $X_{s}$ are generated from the independent $V_{k}$ components under Model $1 .{ }^{22}$ Various distributions are considered that require different approaches for the first-step estimation of $\phi_{V_{3}}$. In the normal and laplace case, $V_{3}$ is symmetrically distributed about zero and $\phi_{V_{3}}$ is real-valued. The other distributions considered correspond to situations where $\phi_{V_{3}}$ is non-vanishing but complex-valued, requiring to estimate separately the modulus and argument of $\phi_{V_{3}}$ : poisson, geometric and gamma.

To measure the distance between the estimator and its target distribution function (density or probability mass function), I use the mean integrated squared error (MISE) defined as:

$$
\operatorname{MISE}_{\widehat{f}_{X_{1}, X_{2}}}=\mathrm{E} \iint\left\{\widehat{f}_{X_{1}, X_{2}}-f_{X_{1}, X_{2}}\right\}^{2} d t d u \text {. }
$$

\footnotetext{
${ }^{22}$ The joint distributions of $X_{1}, X_{2}$ are derived from the assumptions on the $V_{k}$ components using the symbolic algebra package Mathematica.
} 
For each distributional case, I compute the empirical truncated MISE of $\widehat{f}_{X_{1}, X_{2}}$ as well as its bias/variance decomposition, over 100 replications. Truncated MISE is calculated on restricted ranges of $X_{1}$ and $X_{2}$, that are chosen so as to comprise 99.7 percent of the data (in the normal case, they correspond to three standard deviations away from the mean). In addition to the mean I also estimate the median ISE, as it is less sensitive to extreme values that can be encountered in practice. Finally, to assess the quality of the first step estimators, I provide results on the mean and median ISE of $\widehat{\phi}_{V_{3}}$. Four different sample sizes are examined: 100, 250, 500 and 1000 (the three samples $\left\{X_{1}\right\},\left\{X_{2}\right\}$ and $\left\{X_{s}\right\}$ having identical sizes).

\subsubsection{Simulation results}

The estimators performance for a range of values of the truncation parameters $T$ and $U$ are examined. Detailed simulation results are reported in Tables 2.1 to 2.7 for the normal, poisson and gamma cases. For these as well as additional distributional cases,

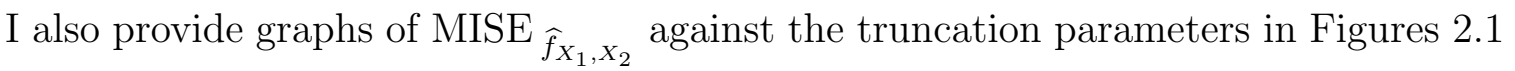
to 2.6 .

Density estimation works very well in the normal and Laplace cases (Tables 2.1 and 2.2, and Figures 2.1 and 2.2), for truncation parameters that are neither too small nor too large. ${ }^{23}$ Too much truncation brings too much information loss, while too little truncation let the noise at the tail of the joint $\mathrm{CF}$ of $X_{1}, X_{2}$ disturb the final distribution estimate. $\operatorname{MISE}_{\widehat{f}_{X_{1}, X_{2}}}$ and the squared bias are both U-shaped, decreasing then increasing with the truncation parameters, while variance increases with the truncation parameters. Discrepancies between the mean and median ISE increase with the truncation parameters, for both $\widehat{f}_{X_{1}, X_{2}}$ and $\widehat{\phi}_{V_{3}}$. This reflects the augmenting difficulty to accurately estimate $\phi_{V_{3}}$ on increasingly long supports. We observe that all MISE and MedISE measures reduce with the sample size. Another

\footnotetext{
${ }^{23}$ Only a few cases are shown here, but the same is true for variants where $X_{1}$ and $X_{2}$ have non-zero means or do not have the same distribution.
} 
consequence of larger sample size is that the range of $T, U$ values for which $\mathrm{MISE}_{\widehat{f}_{X_{1}, X_{2}}}$ is relatively insensitive to truncation parameters is wider, as seen in Figures 2.1 and 2.2 .

Density estimation appears somewhat more difficult in the case of variables that are not symmetric around zero. Detailed simulation results are provided for the poisson and gamma cases. This is not surprising given that the estimation of a complex-valued $\phi_{V_{3}}$ function is more involved. Comparing results across tables, it can be seen that better performance is obtained on less severely skewed data. MISE ${\widehat{f_{X_{1}}, X_{2}}}_{\mathrm{A}}$ is again U-shaped, decreasing then increasing with the truncation parameters. The observed sensitivity of the estimation procedure to the truncation calls for a datadriven selection procedure for $T$ and $U$, which we discuss next.

\subsubsection{Truncation selection}

In this section I propose a data-driven selection procedure for the truncation parameters $U$ and T. Adapted from Diggle and Hall (1993), it is based on the estimation of an approximation of the asymptotic MISE of $\widehat{f}_{X_{1}, X_{2}}$. My truncation point selector therefore resembles plug-in type of bandwidth selectors, as proposed by Ruppert et al. (1995) for kernel density estimation and adapted to the deconvolving kernel density estimator in the measurement error context in Delaigle and Gijbels $(2002,2004) .{ }^{24}$

Given the distance criterion introduced above, the ideal truncation points are defined as $\left\{T_{o p t}, U_{o p t}\right\}=\arg \min _{\{T, U\}} \operatorname{MISE}_{\widehat{f}_{X_{1}, X_{2}}}\left(x_{1}, x_{2} ; T, U\right)$. To choose truncation parameters based on the data I first derive an asymptotic approximation of the MISE, which I then estimate using non-parametric estimates of the unknown quantities. Details of the derivation for the case in which all the variables are symmetrically distributed are provided in Section C.3 of the Appendix. Extension to the general

\footnotetext{
${ }^{24} \mathrm{My}$ proposed second-step estimator is a particular case of the deconvolution kernel density estimator. Truncation points of the truncated inverse Fourier transformation (2.55) are therefore closely related to bandwidth parameters in kernel smoothing methods.
} 
case is left for future work.

Table 2.8 presents estimated optimal truncation parameters obtained for various normal distribution specifications and sample size. These optimal values are systematically higher than the truncation values minimizing the truncated MISE as observed in Tables 2.1 and 2.2. However, the former and the latter present similar patterns: first, they increase with the sample size; second, they decrease as the variance of $V_{3}$ increase. Figures 2.7 to 2.10 depict the true density $f_{X_{1}, X_{2}}$ along with four out of 100 replicated estimates that correspond to the minimum value and the first, second and third quartile of the 100 calculated ISEs, when the optimal truncation parameters of Table 2.8 are used. We see that more precise estimates are observed with a larger sample size. From these figures as well as the three-dimensional pictures in Figures 2.11 and 2.12, it seems that the proposed practical selection procedure as well as the employed estimator perform quite well in the normal case.

\subsubsection{Practical implementation}

\subsubsection{Non-parametric sieve regression}

Polynomial regression is used to estimate $\phi_{V_{3}}(t)$ as described in Section 2.5.1. Depending on the distribution of $V_{3}$, the $\mathrm{CF} \phi_{V_{3}}(t)$ has properties that must be taken into account in the estimation. These properties can also allow focusing the estimation effort on a restricted range of the support of $\phi_{V_{3}}(t)$.

Property 1: A distribution function is symmetric around zero if and only if its characteristic function is real and even (Ushakov, 1999, Th. 1.1.8.).

Property 2: For any characteristic function $\phi(t), \phi(-t)=\overline{\phi(t)}$, where the bar denotes the complex conjugate (Lukacs, 1970, Th. 2.1.1.).

Property 3: The characteristic function of a discrete, integer-valued random variable is periodic with period $2 \pi$ (Ushakov, 1999, p.111). 
It follows from Property 1 that, in the normal case, the estimated $\phi_{V_{3}}(t)$ must be constrained to be even (such that $\phi_{V_{3}}(-t)=\phi_{V_{3}}(t)$ ). Alternatively, $\phi_{V_{3}}(t)$ can be estimated on half of its support, then extrapolated to the other half using the evenness property. It also follows that if they have non-zero means, variables $X_{1}, X_{2}$ and $X_{s}$ should be demeaned to ensure that $V_{3}$ is centered on zero.

In the gamma and poisson cases, $V_{3}$ is not symmetrically distributed and $\phi_{V_{3}}$ is complex-valued. This requires to estimate its modulus and argument separately. It follows from Property 2 that $\operatorname{Mod}\left(\phi_{V_{3}}(-t)\right)=\operatorname{Mod}\left(\phi_{V_{3}}(t)\right)$ and $\operatorname{Arg}\left(\phi_{V_{3}}(-t)\right)=$ $-\operatorname{Arg}\left(\phi_{V_{3}}(t)\right)$. In words, the modulus of $\phi_{V_{3}}(t)$ is even and its argument is odd. Again, these properties must be accounted for in the estimation. Finally, in the poisson case and following Property 3 , it suffices to estimate $\phi_{V_{3}}(t)$ on any interval of length $2 \pi$.

To implement sieve regression, the order of the sieve (the number of regressors) must be selected. Ideally, it must be chosen so as to minimize the MISE of the sieve estimator. Hansen (2014) discusses selection by cross-validation and shows that for least squares estimation, cross-validation is a quite simple calculation that does not require cumbersome leave-one-out operations. I follow this approach whenever possible, and also assess the sensitivity of the final results to changes in the number of polynomials included in the non-parametric regressions.

\subsubsection{Fourier inversion}

The (truncated) two-dimensional Fourier inversion necessary to recover the joint den-

sity function $f_{X_{1}, X_{2}}$ from the joint $\mathrm{CF}$ is highly computationally intensive. I make use of the Fast Fourier transform (FFT) algorithm developed by Cooley and Tukey (1965) to efficiently compute sum approximations of the integrals in Equation (2.55).

All computations are performed in $\mathrm{R}$ using the $f f t$ command. This function computes the discrete version of the Fourier transform (FT) of an array using the FFT 
algorithm, where the (two-dimensional) FT of the function $\zeta(t, u)$ is defined as

$$
F T(\zeta)(x, y)=\int_{-\infty}^{\infty} \int_{-\infty}^{\infty} \zeta(t, u) e^{-i 2 \pi(t x+u y)} d t d u
$$

Some preliminary transformations of Equation (2.55) are necessary before we can apply R's fft function. Details on the computational algorithm are available in Section C.4 of the Appendix.

In the case of lattice distributions with span $\lambda$ (taking values of the form $a \pm k \lambda$, $k=0, \pm 1, \pm 2, \ldots)$ such as the Poisson distribution, the inversion formula recovering the joint probability mass function (PMF) from the joint $\mathrm{CF}$ is

$$
\operatorname{Pr}\left(X_{1}=x_{1}, X_{2}=x_{2}\right)=\frac{\lambda^{2}}{(2 \pi)^{2}} \int_{0}^{2 \pi / \lambda} \int_{0}^{2 \pi / \lambda} e^{-i\left(t x_{1}+u x_{2}\right)} \phi_{X_{1}, X_{2}}(t, u) d t d u
$$

for every integer $k$; see Abate and Whitt (1992). Although the inversion integrals concentrate on bounded intervals, in practice some (upper) truncation is necessary, as can be seen from the simulation results in the poisson case. Cases of discrete but not lattice-valued variables cannot in general be handled using a Fourier inversionbased approach. ${ }^{25}$ Also note that in the case of discrete variables with known finite support (e.g. binary variables), the modelling assumption of independence between the $V_{k}$ components becomes untenable.

\footnotetext{
${ }^{25}$ Some studies adapt methods from the deconvolution literature to recover mixtures of discrete and continuous distributions in the context of measurement error models; see Van Es et al. (2008), Lee et al. (2010) and Lee et al. (2013). Other approaches that could be used to estimate the joint PMF in case of discrete random variables -with infinite support- are discussed for example in Devroye and Wise (1979), and Delaigle and Hall (2016).
} 


\subsection{Conclusion}

In this chapter, I consider a sample combination framework that does not rely on common variables, and is encountered in real data. I establish non-parametric identification of the joint distribution of $X_{1}, X_{2}$ when no bivariate sample of $X_{1}, X_{2}$ is observed, by combining the marginal distributions of $X_{1}$ and $X_{2}$, and the marginal distribution of their sum $X_{1}+X_{2}$. A consistent two-step estimation procedure is proposed and shown to behave relatively well in small-sample simulations.

There are several directions in which this work could be developed. First, the formal identification proof is not constructive, insofar as the proof of Lemma 1 does not express the underlying function $\phi_{V_{3}}$ directly in terms of observable quantities. A constructive identification proof could provide a consistent non-parametric estimator that does not rely on sieve approximation. One potential avenue, following Meister et al. (2010), was briefly mentioned at the end of Section 2.5.1. Second, all models discussed in this chapter are cross-sectional. An interesting extension for future work would be to include temporal dynamics using panel data models. With regard to estimation, performance gains can be expected from further enhancing or fine-tuning the procedures. Finally, a truncation parameter selection procedure has still to be derived in the general case when the variables are not all symmetrically distributed. 


\section{References}

Abate, J. And W. Whitt (1992): "The Fourier-series method for inverting transforms of probability distributions," Queueing systems, 10, 5-87.

Balakrishnan, N. And C. D. LAi (2009): Continuous bivariate distributions, New York ; London : Springer, 2nd ed.

Bernard, C. And D. MCLeish (2015): "Algorithms for Finding Copulas Minimizing Convex Functions of Sums," .

Carroll, R. J. And P. Hall (1988): "Optimal rates of convergence for deconvolving a density," Journal of the American Statistical Association, 83, 1184-1186.

Cooley, J. W. And J. W. Tukey (1965): "An algorithm for the machine calculation of complex Fourier series," Mathematics of computation, 19, 297-301.

Cramér, H. (1939): "On the representation of a function by certain Fourier integrals," Transactions of the American Mathematical Society, 46, 191-201.

Dall'Aglio, G. (1972): "Fréchet classes and compatibility of distribution functions," Symposia mathematica, 9, 131-150.

Delaigle, A. (2014a): "Nonparametric kernel methods for curve estimation and measurement errors," Proceedings of the International Astronomical Union, 10, $28-39$.

(2014b): "Nonparametric Kernel Methods with Errors-in-Variables: Constructing Estimators, Computing them, and Avoiding Common Mistakes," Australian $\&$ New Zealand Journal of Statistics, 56, 105-124.

Delaigle, A. AND I. Gijbels (2002): "Estimation of integrated squared density derivatives from a contaminated sample," Journal of the Royal Statistical Society: Series B (Statistical Methodology), 64, 869-886.

(2004): "Practical bandwidth selection in deconvolution kernel density estimation," Computational statistics \& data analysis, 45, 249-267.

Delaigle, A. And P. Hall (2016): "Methodology for non-parametric deconvolution when the error distribution is unknown," Journal of the Royal Statistical Society: Series B (Statistical Methodology), 78, 231-252.

Delaigle, A., P. Hall, And A. Meister (2008): "On deconvolution with repeated measurements," The Annals of Statistics, 36, 665-685.

Devroye, L. P. And G. L. Wise (1979): "On the recovery of discrete probability densities from imperfect measurements," Journal of the Franklin Institute, 307, $1-20$. 
D'Haultfoeuille, X. (2011): "On the completeness condition in nonparametric instrumental problems," Econometric Theory, 27, 460-471.

Diggle, P. J. And P. Hall (1993): "A Fourier approach to nonparametric deconvolution of a density estimate," Journal of the Royal Statistical Society. Series B (Methodological), 55, 523-531.

Dobra, A. And S. Fienberg (2001): "Bounds for cell entries in contingency tables induced by fixed marginal totals," Statistical Journal of the United Nations Economic Commission for Europe, 18, 363-371.

Embrechts, P., A. McNeil, And D. Straumann (2002): "Correlation and dependence in risk management: properties and pitfalls," in Risk management: value at risk and beyond, ed. by M. Dempster, Cambridge University Press, Cambridge, $176-223$.

Evdokimov, K. (2010): "Identification and estimation of a nonparametric panel data model with unobserved heterogeneity," Working Paper.

Evdokimov, K. And H. White (2012): "Some Extensions of a Lemma of Kotlarski," Econometric Theory, 28, 925-932.

Fan, Y. And A. J. Patton (2014): "Copulas in Econometrics," Annual Review of Economics, 6, 179-200.

Firpo, S., A. F. Galvao, And S. Song (2015): "Measurement Errors in Quantile Regression Models," Unpublished.

Fisher, N. AND P. SEN, eds. (2012): The collected works of Wassily Hoeffding, Springer Science \& Business Media.

FrÉchet, M. (1951): "Sur les tableaux de correlation dont les marges sont donnees," Ann. Univ. Lyon Sci. Sect. A, 14, 53-77.

Hall, P. And A. Meister (2007): "A ridge-parameter approach to deconvolution," The Annals of Statistics, 35, 1535-1558.

HAnsen, B. (2014): "Nonparametric sieve regression: Least squares, averaging least squares, and cross-validation." in Handbook of Applied Nonparametric and Semiparametric Econometrics and Statistics, ed. by L. S. . A. U. J. S. Racine, Oxford University Press.

Horowitz, J. L. (2014): "Ill-posed inverse problems in economics," Annu. Rev. Econ., 6, 21-51.

Horowitz, J. L. And M. Markatou (1996): "Semiparametric estimation of regression models for panel data," The Review of Economic Studies, 63, 145-168.

Ignatenko, G. (2010): "Nonparametric density estimation in finance and insurance," Master thesis, University of Amsterdam, Netherlands. 
Kotlarski, I. (1967): "On characterizing the gamma and the normal distribution," Pacific Journal of Mathematics, 20, 69-76.

Lee, M., P. Hall, H. Shen, J. S. Marron, J. Tolle, and C. Burch (2013): "Deconvolution estimation of mixture distributions with boundaries," Electronic journal of statistics, 7,323 .

Lee, M., H. Shen, C. Burch, and J. S. Marron (2010): "Direct deconvolution density estimation of a mixture distribution motivated by mutation effects distribution," Journal of nonparametric statistics, 22, 1-22.

Linton, O. AND Y.-J. WhANG (2002): "Nonparametric estimation with aggregated data," Econometric Theory, 18, 420-468.

LiU, M. C. AND R. L. TAYlor (1989): "A consistent nonparametric density estimator for the deconvolution problem," Canadian Journal of Statistics, 17, 427-438.

LukACs, E. (1970): Characteristics functions, Griffin, London.

Meister, A. (2009): "Deconvolution Problems in Nonparametric Statistics," in Lecture Notes in Statistics 193, Springer Berlin Heidelberg.

Meister, A., U. Stadtmüller, And C. Wagner (2010): "Density deconvolution in a two-level heteroscedastic model with unknown error density," Electron. J. Statist., 4, 36-57.

Nelsen, R. B. (2013): An introduction to copulas, vol. 139, Springer Science \& Business Media.

Nelsen, R. B., J. J. Q. Molina, J. A. R. Lallena, and M. Ú. Flores (2004): "Best-possible bounds on sets of bivariate distribution functions," Journal of Multivariate Analysis, 90, 348-358.

Nelsen, R. B., J. J. Quesada-Molina, J. A. RodriÍGuez-Lallena, And M. UBEDA-FLORES (2001): "Bounds on bivariate distribution functions with given margins and measures of association," Communications in Statistics-Theory and Methods, 30, 1055-1062.

PiterbarG, V. (2011): "Spread options, Farkass lemma and linear programming," Risk, 24, 61.

Puccetti, G. And R. Wang (2015): "Detecting complete and joint mixability," Journal of Computational and Applied Mathematics, 280, 174-187.

RAO, B. P. (1992): Identifiability in Stochastic Models, New York: Academic Press.

RÉnyi, A. (1950): "On the algebra of distributions," Publ. Math. Debrecen, 1, 135149. 
RIDDER, G. AND Y. Hu (2012): "Estimation of Nonlinear Models with Mismeasured Regressors Using Marginal Information," Journal of Applied Econometrics, 27, 347385 .

Ridder, G. And R. Moffitt (2007): "The Econometrics of Data Combination," in Handbook of Econometrics, ed. by J. J. Heckman and E. E. Leamer, Elsevier, vol. 6, Part B, $5469-5547$.

Ruppert, D., S. J. Sheather, And M. P. Wand (1995): "An effective bandwidth selector for local least squares regression," Journal of the American Statistical Association, 90, 1257-1270.

RÜSCHENDORF, L. AND L. UCKELMANN (2002): "Variance minimization and random variables with constant sum," in Distributions with given marginals and statistical modelling, Springer, 211-222.

Sarabia Alzaga, J. M. And E. Gómez DÉniz (2008): "Construction of multivariate distributions: a review of some recent results," Statistics and Operations Research Transactions, 32, 3-36.

Stefanski, L. A. And R. J. Carroll (1990): "Deconvolving kernel density estimators," Statistics, 21, 169-184.

TANkov, P. (2011): "Improved Fréchet bounds and model-free pricing of multi-asset options," Journal of Applied Probability, 48, 389-403.

Ushakov, N. G. (1999): Selected topics in characteristic functions, VSP, Utrecht, The Netherlands.

Van Es, B., S. Gugushvili, P. SpreiJ, et Al. (2008): "Deconvolution for an atomic distribution," Electronic Journal of Statistics, 2, 265-297.

Vermeulen, F. (2002): "Collective Household Models: Principles and Main Results." Journal of Economic Surveys, 16, 533 - 564.

WAKEFiELD, J. (2004): "Ecological inference for $2 \times 2$ tables," Journal of the Royal Statistical Society: Series A (Statistics in Society), 167, 385-445.

WANG, B. AND R. WANG (2011): "The complete mixability and convex minimization problems with monotone marginal densities," Journal of Multivariate Analysis, $102,1344-1360$.

(2015): "Joint mixability," Mathematics of Operations Research, forthcoming. 


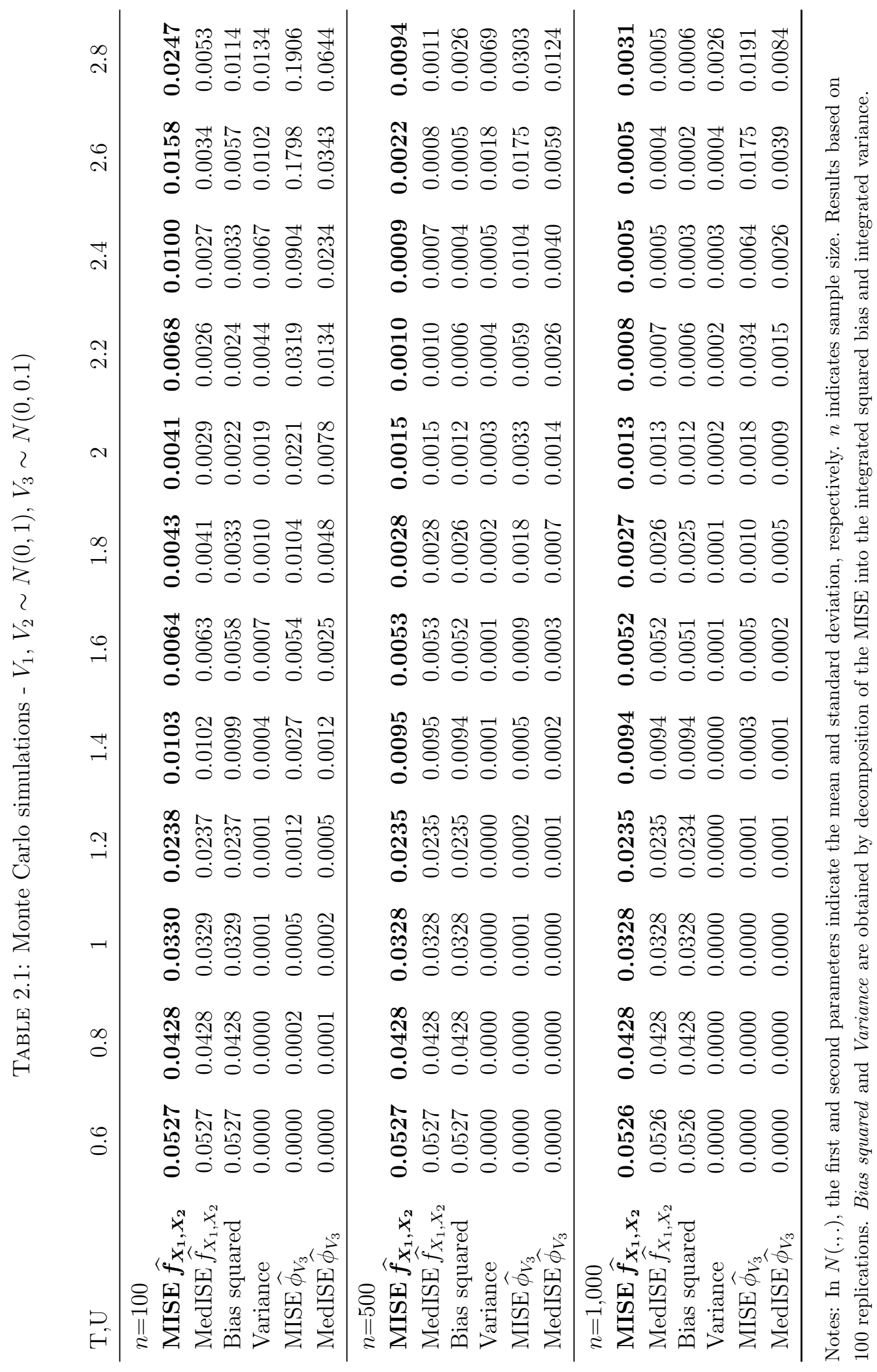




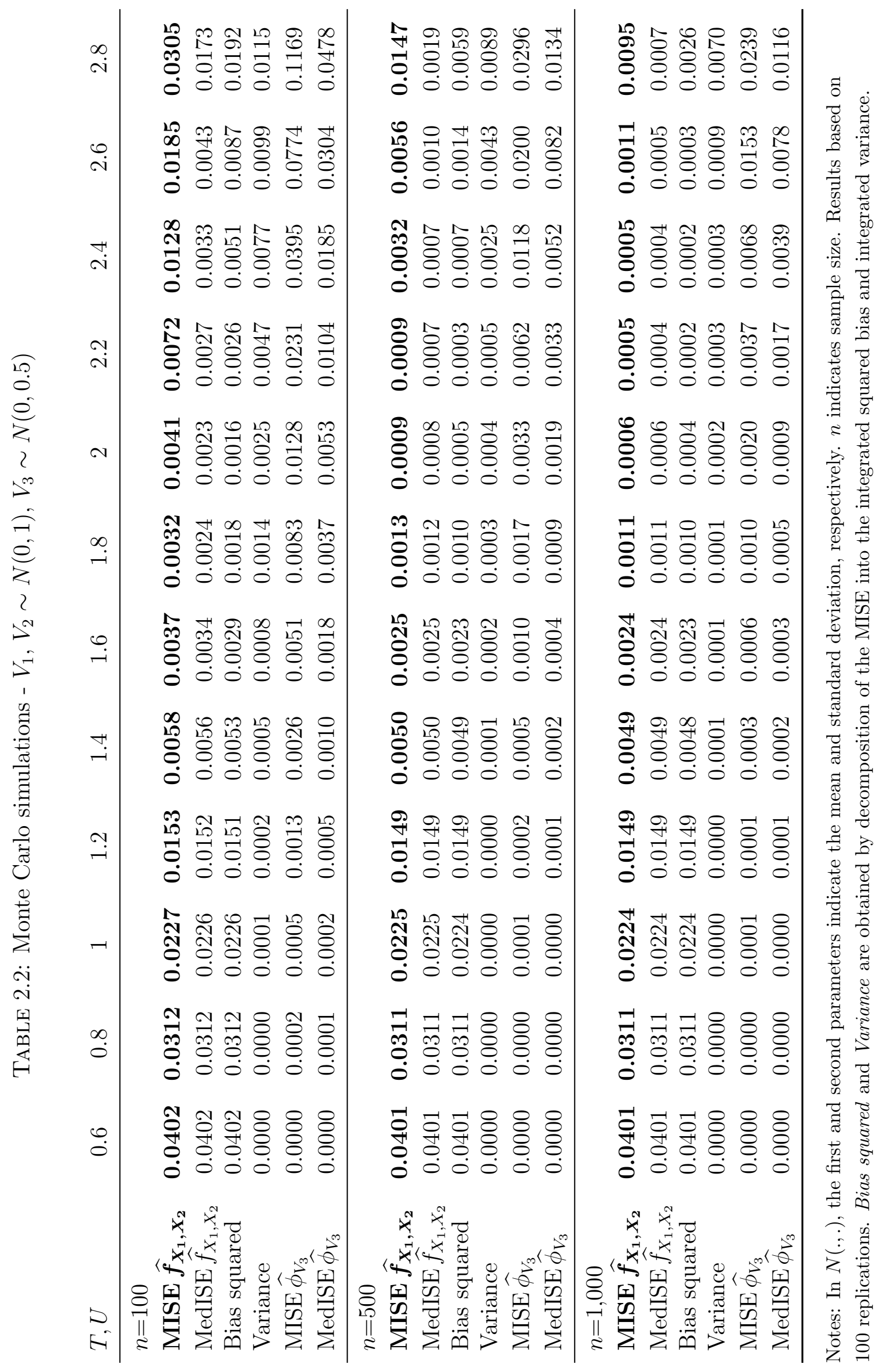




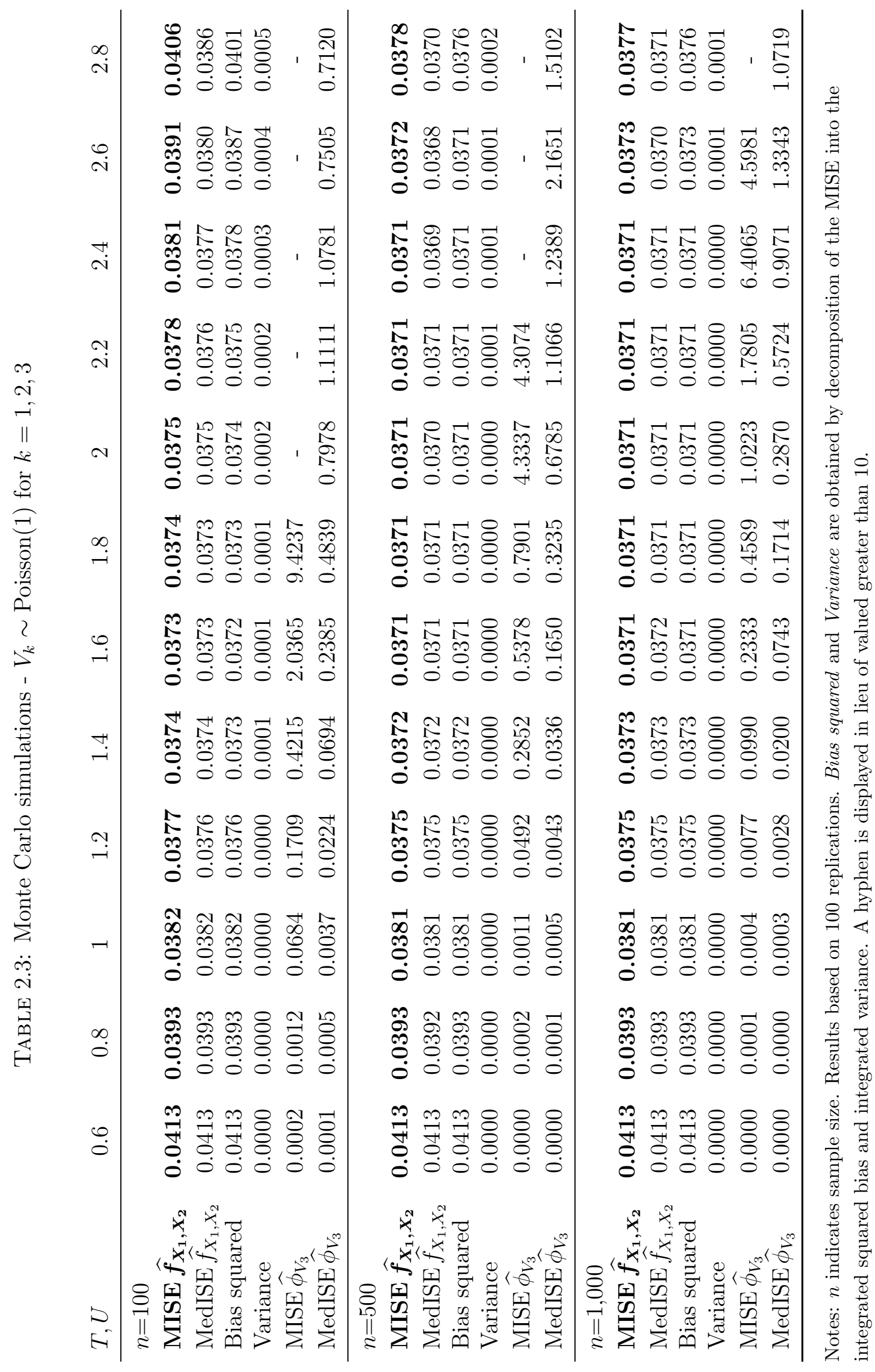




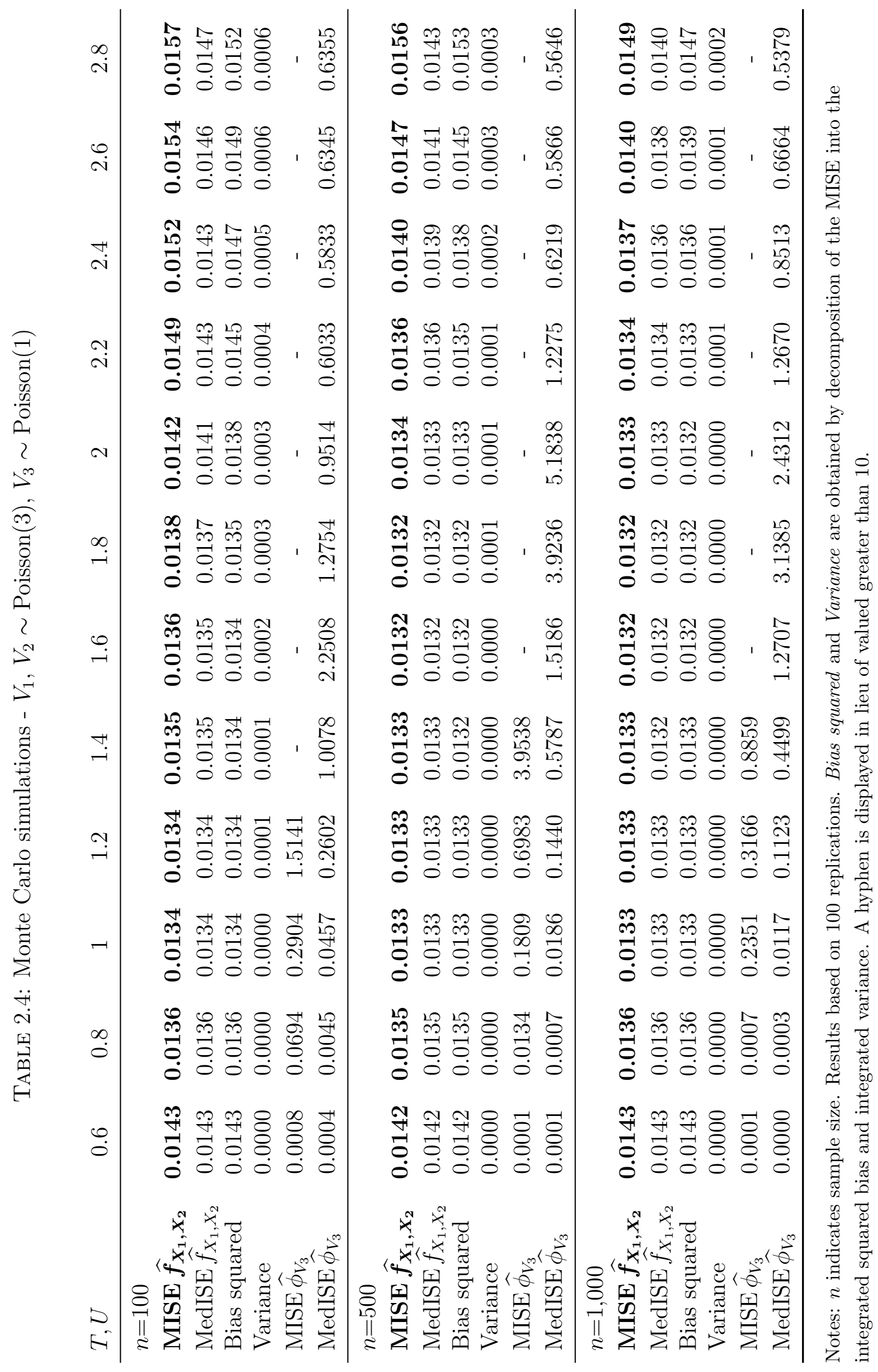




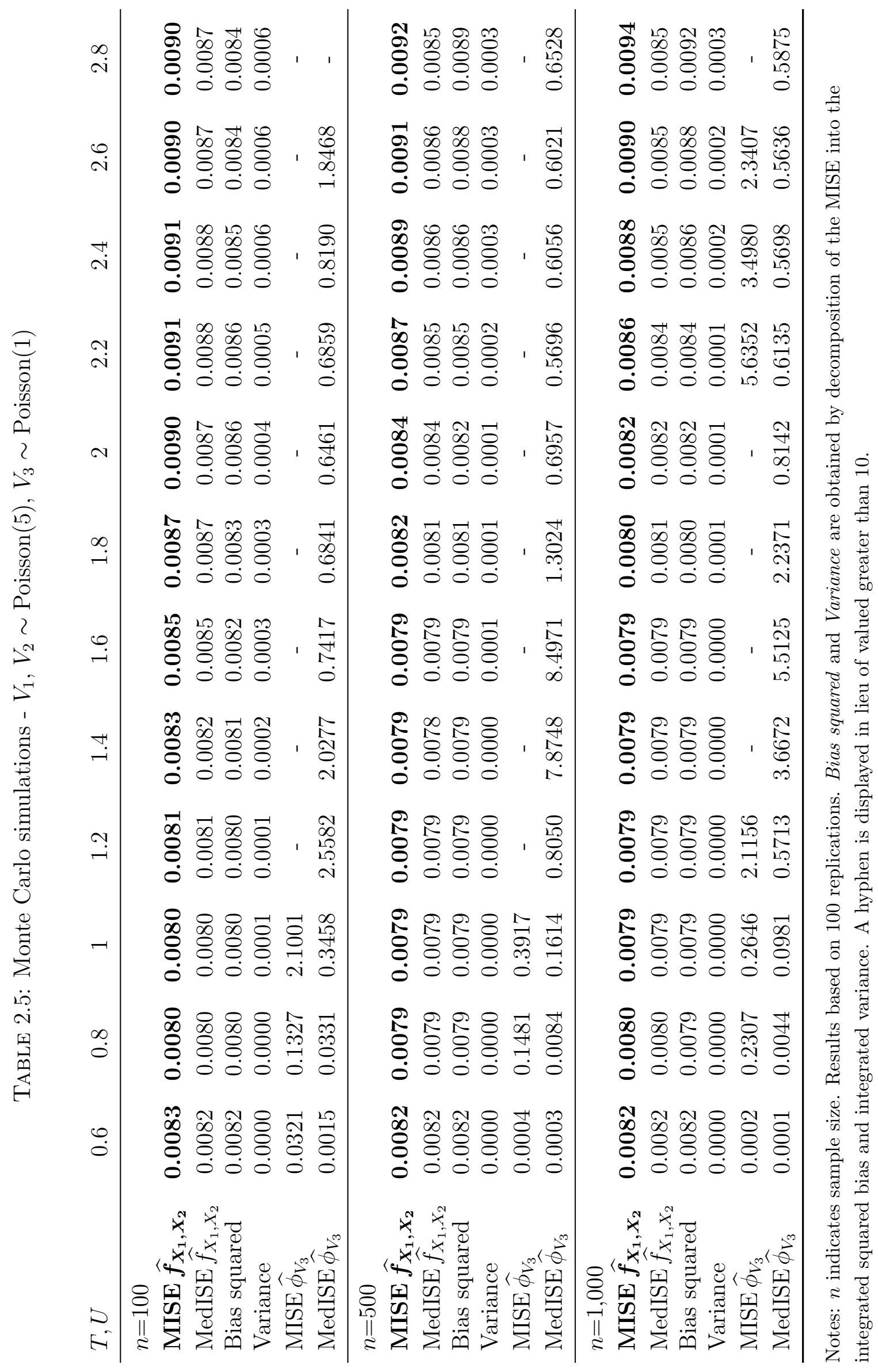




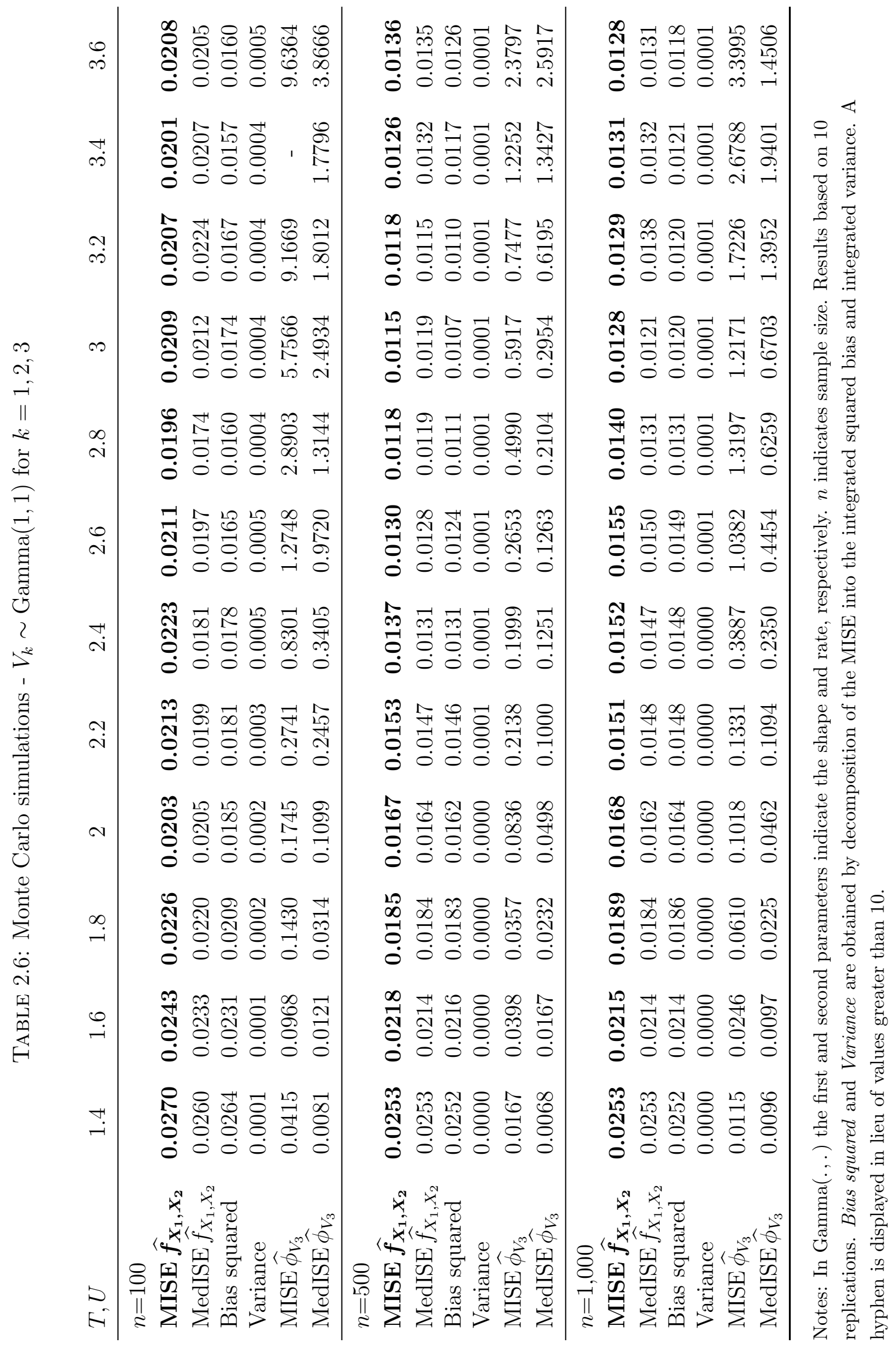




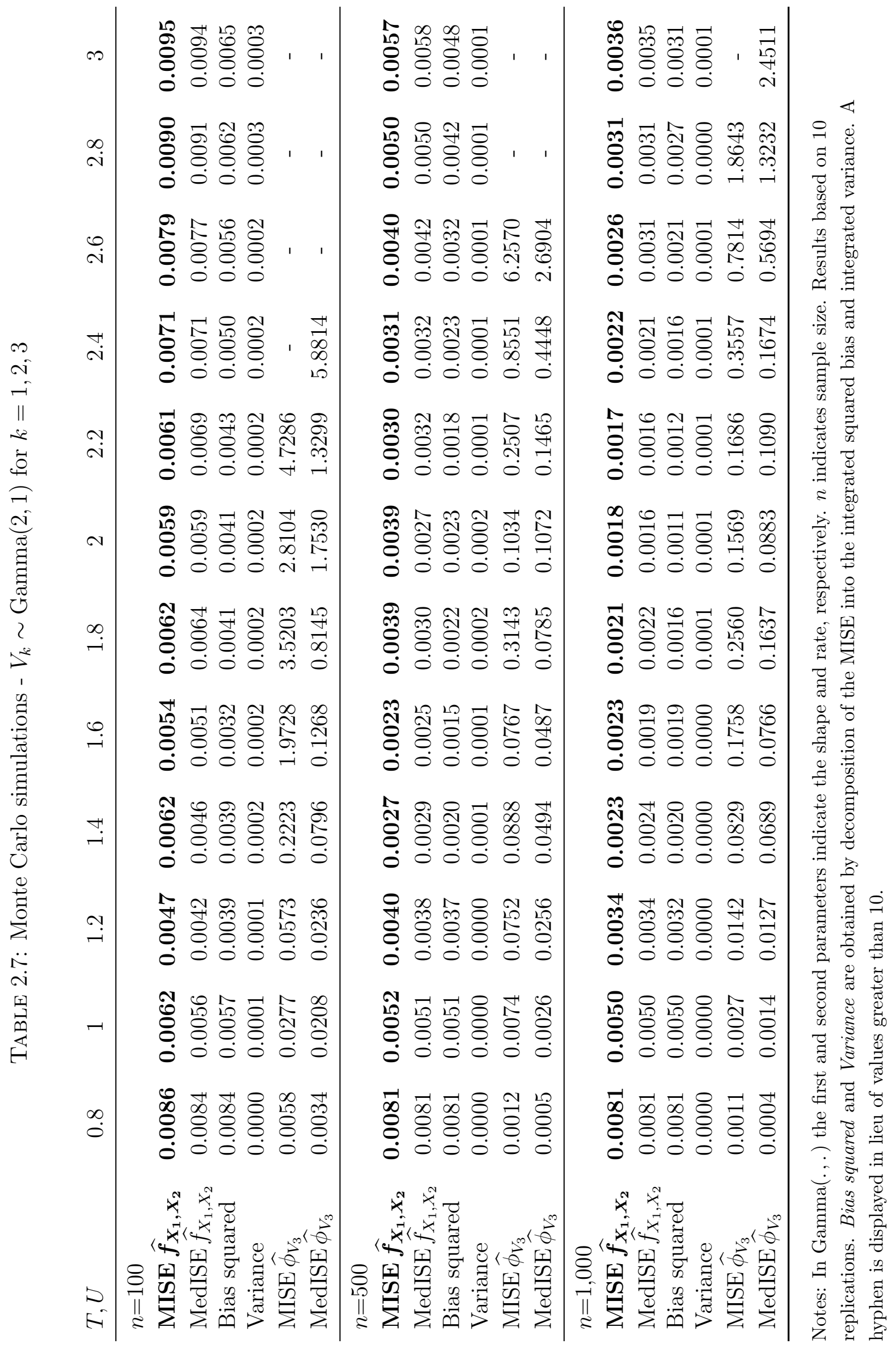


TABLE 2.8: Optimal truncation estimates

\begin{tabular}{lccc} 
& $V_{3} \sim N(0,0.1)$ & $V_{3} \sim N(0,0.5)$ & $V_{3} \sim N(0,1)$ \\
\hline$n=100$ & 2.7 & 2.5 & 2.3 \\
$n=500$ & 2.9 & 2.6 & 2.4 \\
$n=1000$ & 3.3 & 2.7 & 2.8 \\
\hline
\end{tabular}

Notes: Average values obtained over 10 replications. $V_{1}$ and $V_{2} \sim N(0,1)$ 


\section{Figure 2.1: MISE estimates - normal distribution}

(a) $V_{3} \sim N(0,0.1)$

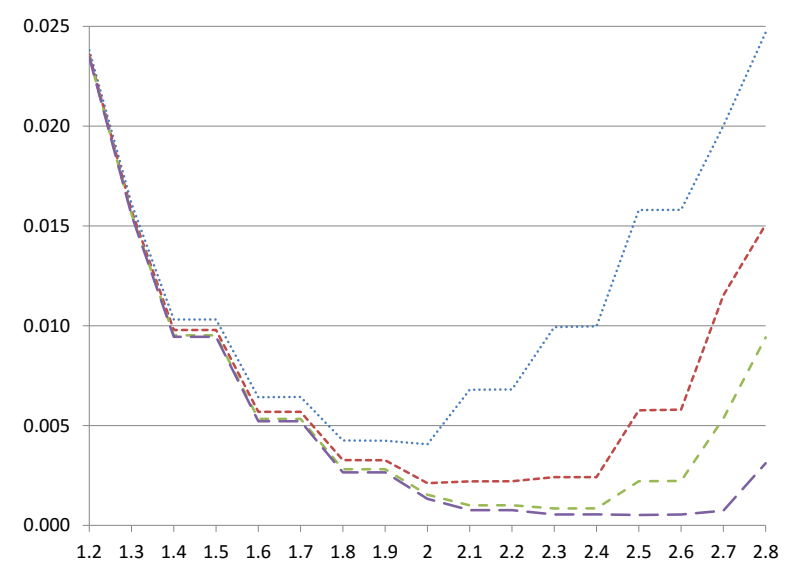

(b) $V_{3} \sim N(0,0.5)$

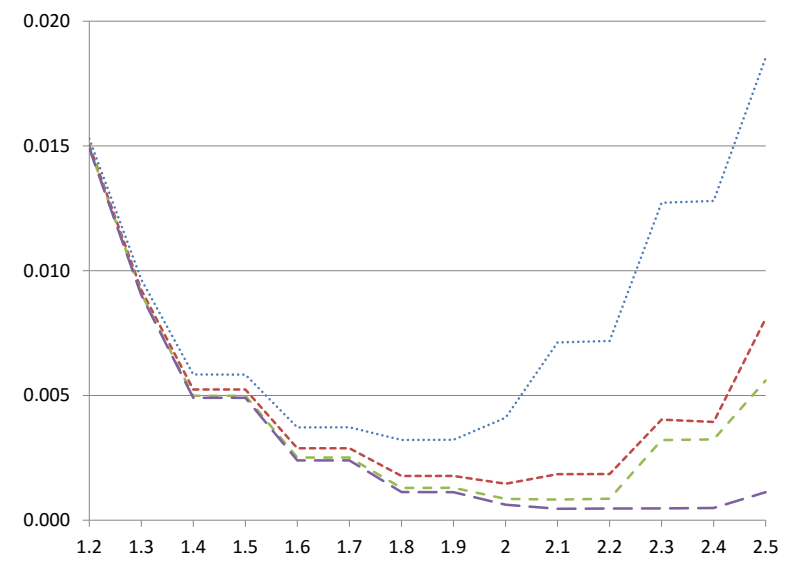

(c) $V_{3} \sim N(0,1)$

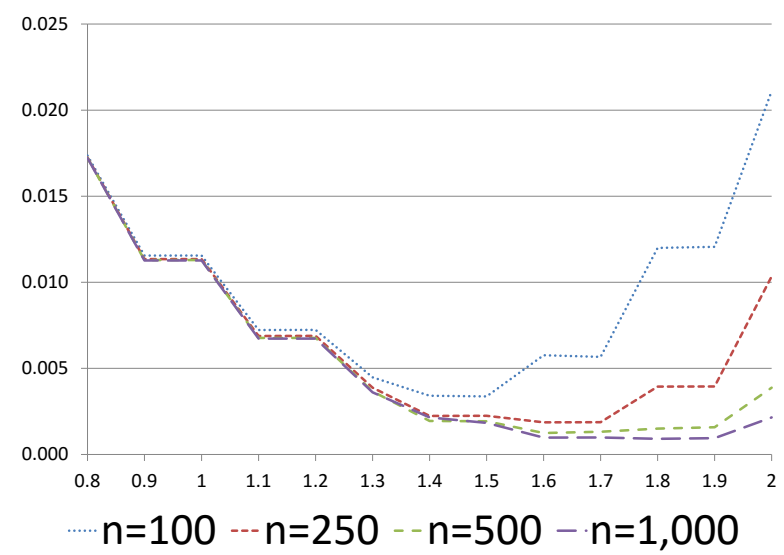

Notes: $V_{1}$ and $V_{2} \sim N(0,1)$ in all three cases. In $N(.,$.$) , the first and second parameters indicate$ the mean and standard deviation, respectively. $n$ indicates sample size. $T=U$ is in the abscissa. Results based on 100 replications. 
Figure 2.2: MISE estimates - Laplace distribution

(a) $V_{k} \sim$ Laplace $(0,0.5)$ for $k=1,2,3$

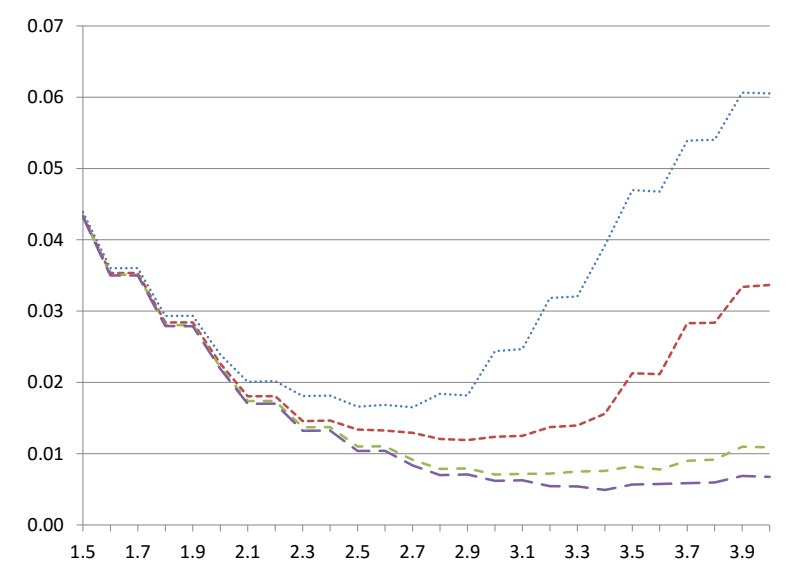

(b) $V_{k} \sim \operatorname{Laplace}(0,1)$ for $k=1,2,3$

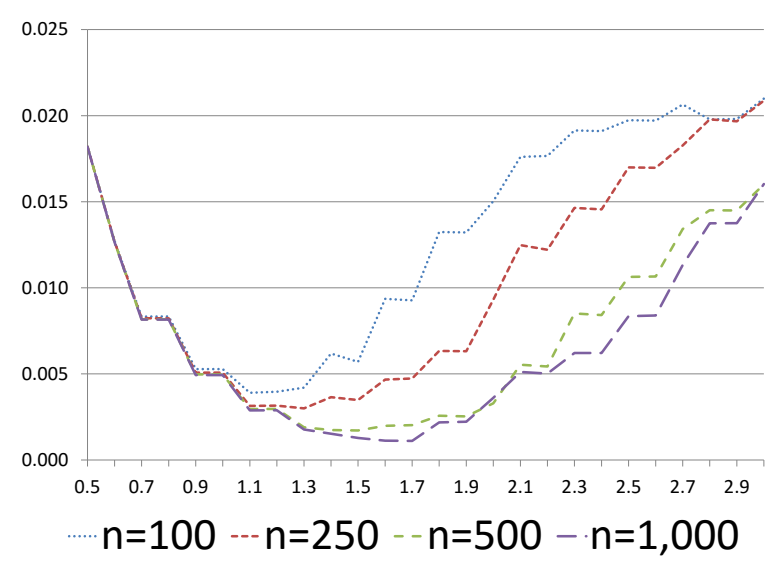

Notes: In Laplace(.,.), the first and second parameters indicate the location and scale, respectively. $n$ indicates sample size. $T=U$ is in the abscissa. Results based on 100 replications. 
Figure 2.3: MISE estimates - bivariate normal distribution

(a) $\left(X_{1}, X_{2}\right) \sim \mathrm{N}\left(\mu_{1}=\mu_{2}=0, \sigma_{1}^{2}=\sigma_{2}^{2}=1.25, \rho=0.2\right)$

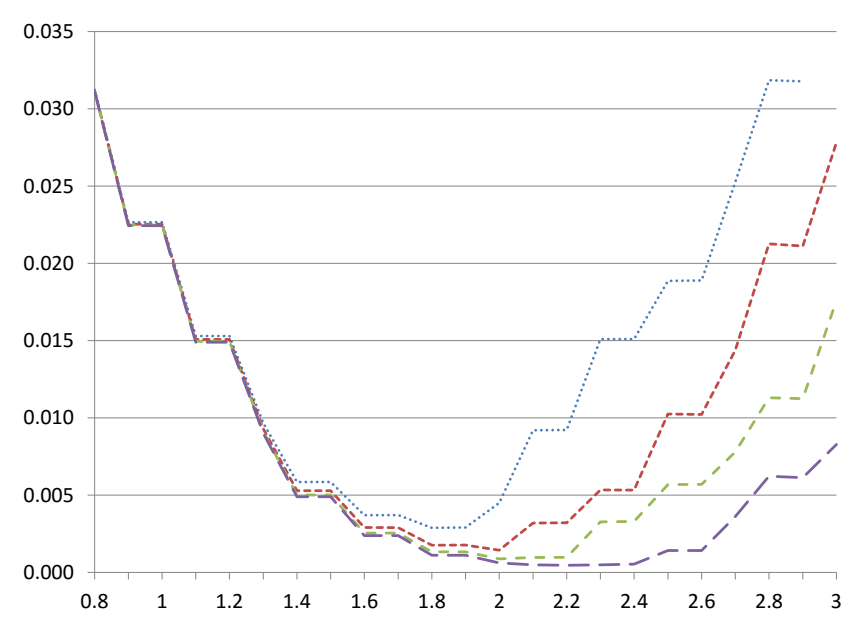

(b) $\left(X_{1}, X_{2}\right) \sim \mathrm{N}\left(\mu_{1}=\mu_{2}=0, \sigma_{1}^{2}=\sigma_{2}^{2}=2, \rho=0.5\right)$

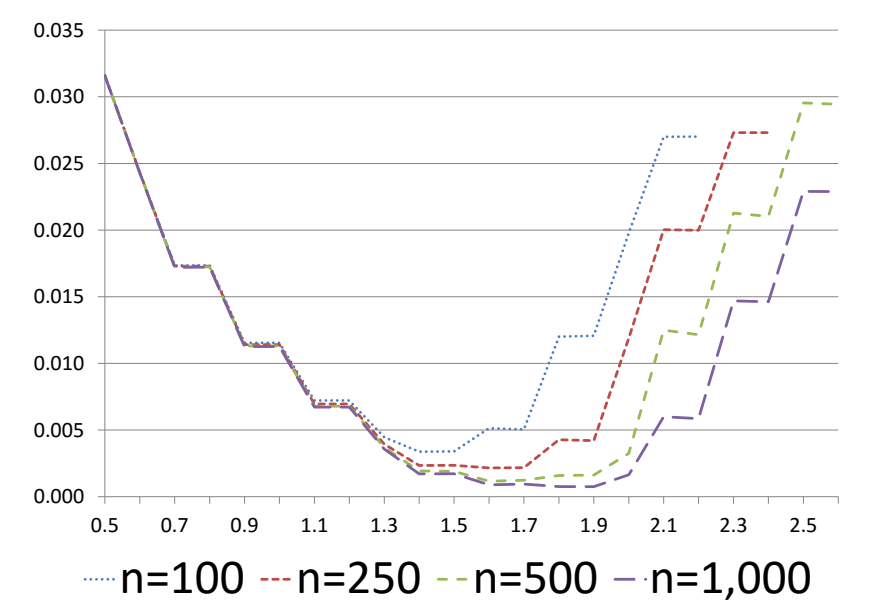

Notes: $X_{1}$ and $X_{2}$ are simulated directly from bivariate normal distributions. $n$ indicates sample size. $T=U$ is in the abscissa. Results based on 100 replications. 
Figure 2.4: MISE estimates - Poisson distribution

(a) $V_{k} \sim \operatorname{Poisson}(1)$ for $k=1,2,3$

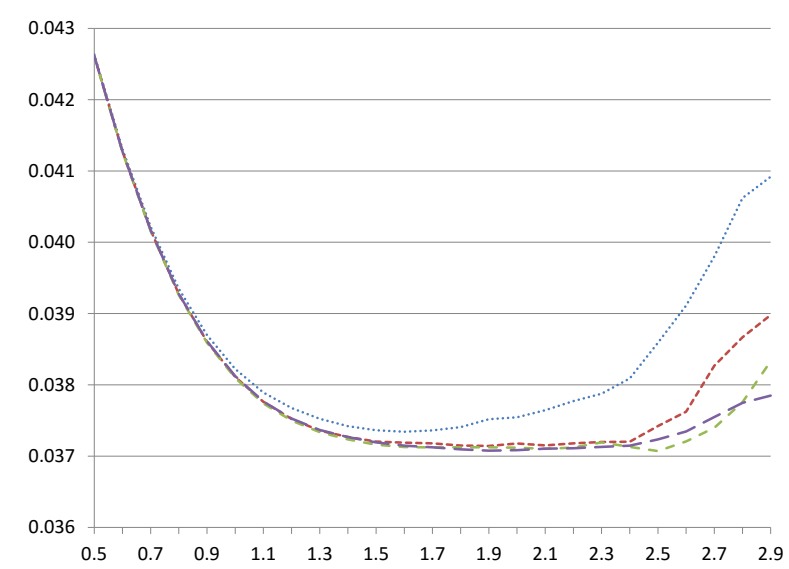

(b) $V_{1}$ and $V_{2} \sim \operatorname{Poisson}(3), V_{3} \sim \operatorname{Poisson}(1)$

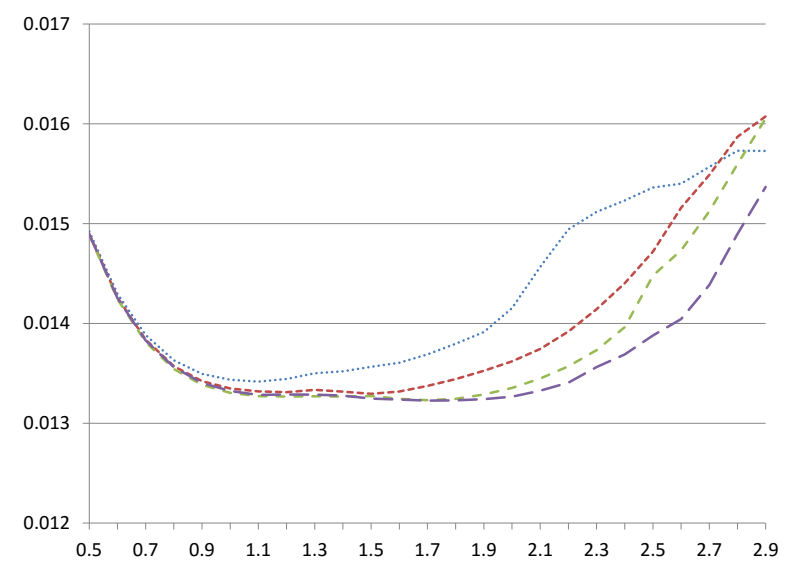

(c) $V_{1}$ and $V_{2} \sim \operatorname{Poisson}(5), V_{3} \sim \operatorname{Poisson}(1)$

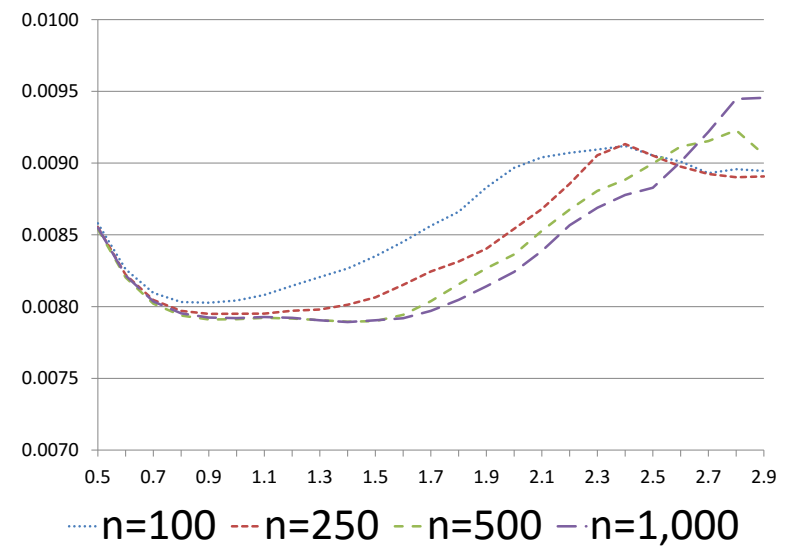

Notes: $n$ indicates sample size. $T=U$ is in the abscissa. Results based on 100 replications. 
Figure 2.5: MISE estimates - gamma distribution

(a) $V_{k} \sim \operatorname{Gamma}(1,1)$ for $k=1,2,3$

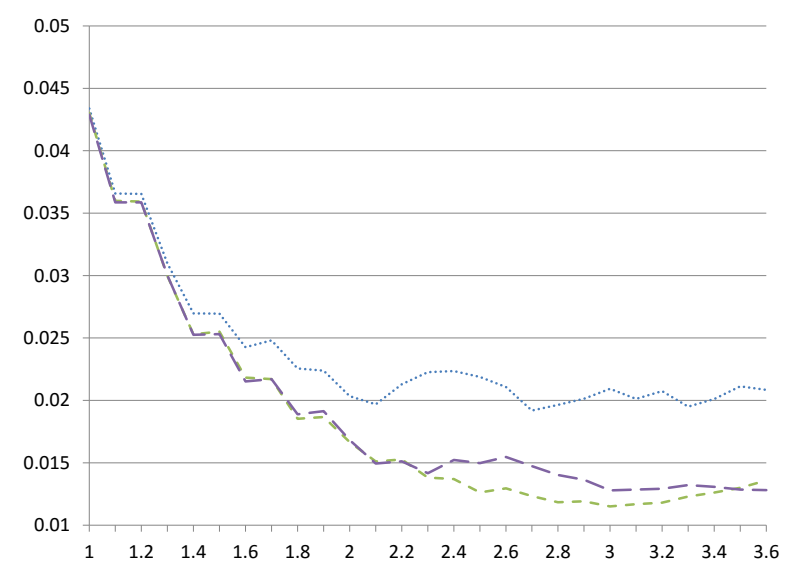

(b) $V_{k} \sim \operatorname{Gamma}(2,1)$ for $k=1,2,3$

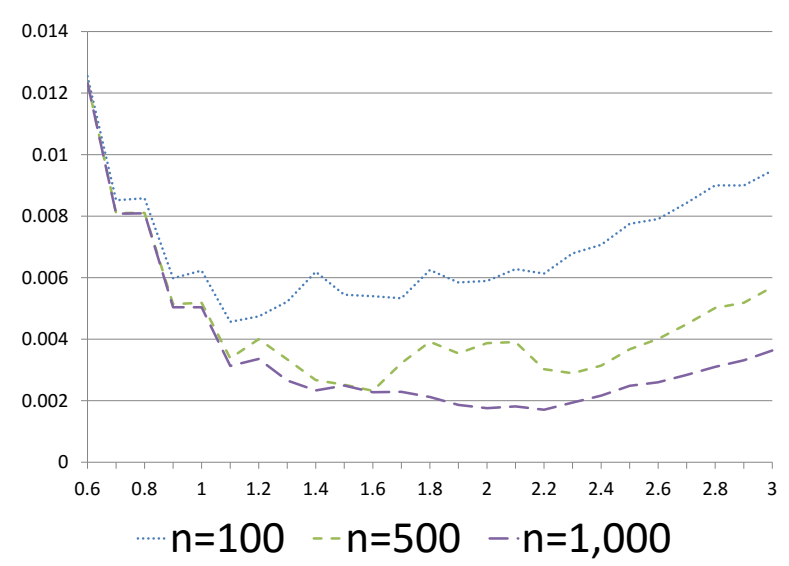

Notes: In Gamma(.,. ) the first and second parameters indicate the shape and rate, respectively. $n$ indicates sample size. $T=U$ is in the abscissa. Results based on 10 replications. 
Figure 2.6: MISE estimates - geometric distribution

(a) Geometric: $V_{k} \sim \mathrm{G}(0.3)$ for $k=1,2,3$

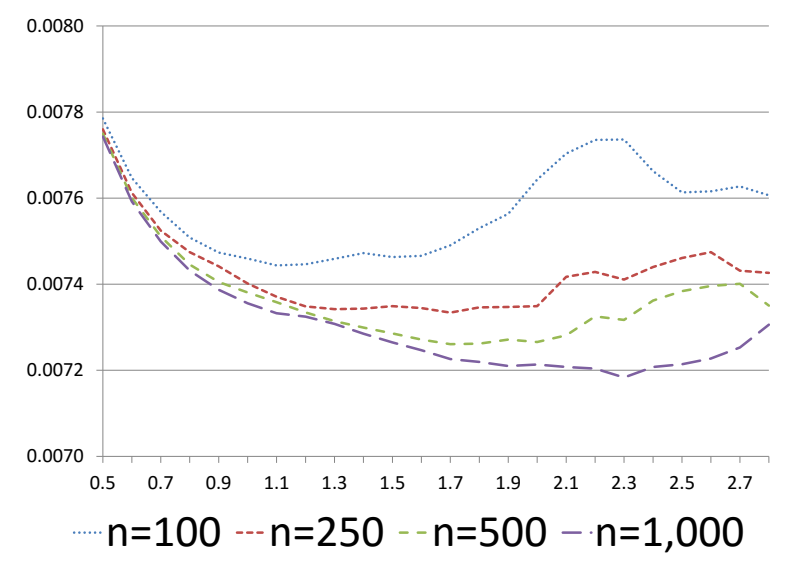

Notes: G denotes the unshifted Geometric distribution (with support starting at zero). $n$ indicates sample size. $T=U$ is in the abscissa. Results based on 100 replications. 
Figure 2.7: Contour plots of $\widehat{f}_{X_{1}, X_{2}}$ - normal case $-V_{3} \sim N(0,0.1)$ and $n=1,000$

(a) Minimum

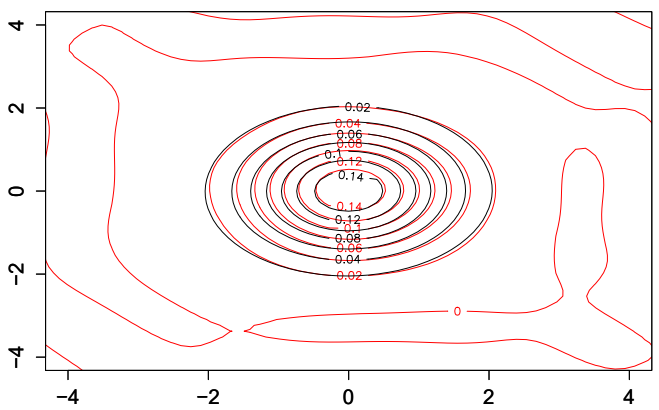

(c) Second quartile

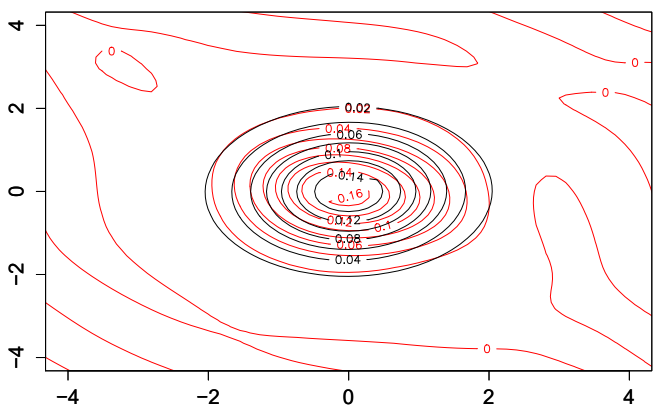

(b) First quartile

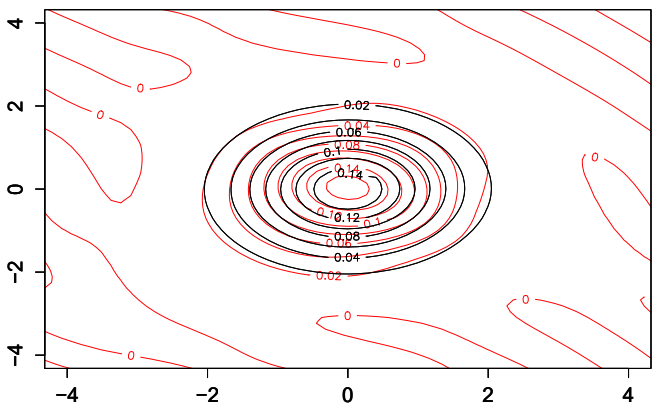

(d) Third quartile

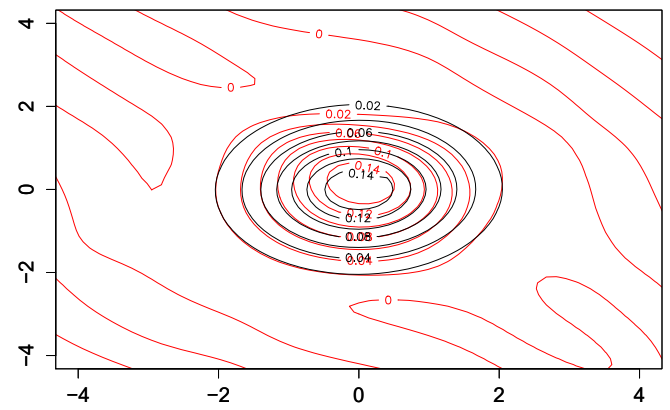

Notes: $V_{1}$ and $V_{2} \sim N(0,1)$. Sample of size $n=1,000$. Target density in black, estimated densities in red: four out of 100 replicated estimates corresponding to the minimum value and the first, second and third quartile of the 100 calculated ISEs. Truncations parameters are $T=U=3.3$. 
Figure 2.8: Contour plots of $\widehat{f}_{X_{1}, X_{2}}$ - normal case $-V_{3} \sim N(0,0.1)$ and $n=100$

(a) Minimum

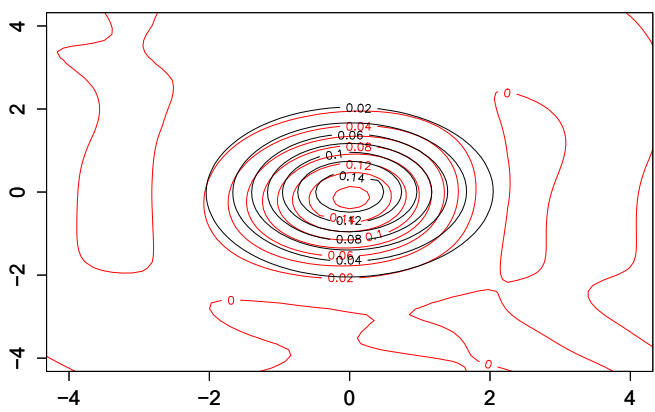

(c) Second quartile

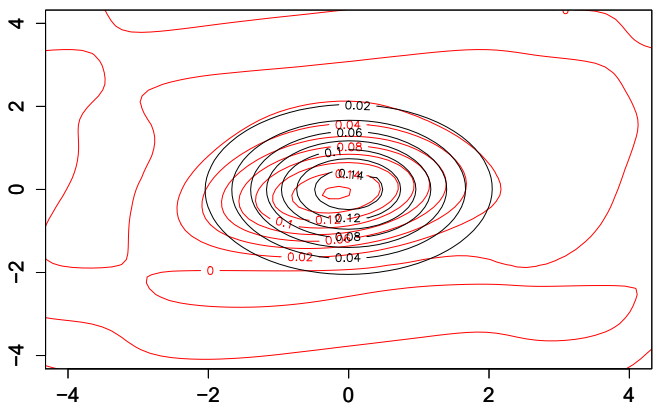

(b) First quartile

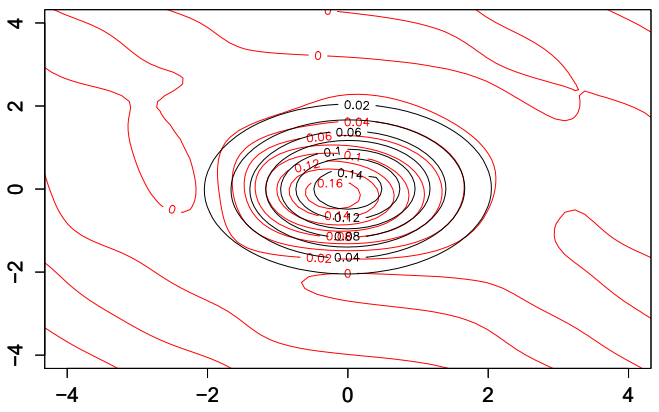

(d) Third quartile

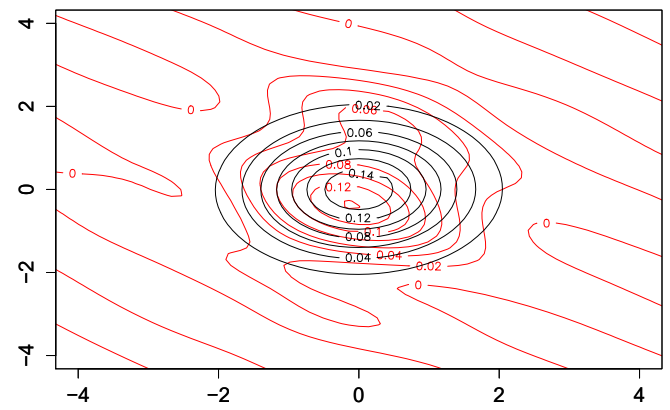

Notes: $V_{1}$ and $V_{2} \sim N(0,1)$. Sample of size $n=100$. Target density in black, estimated densities in red: four out of 100 replicated estimates corresponding to the minimum value and the first, second and third quartile of the 100 calculated ISEs. Truncations parameters are $T=U=2.7$. 
Figure 2.9: Contour plots of $\widehat{f}_{X_{1}, X_{2}}$ - normal case $-V_{3} \sim N(0,0.5)$ and $n=1,000$

(a) Minimum

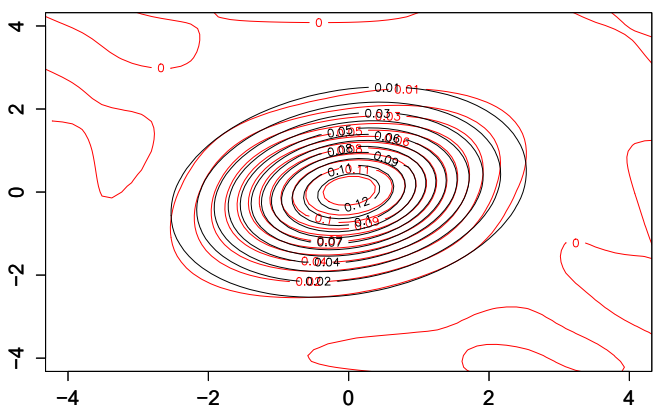

(c) Second quartile

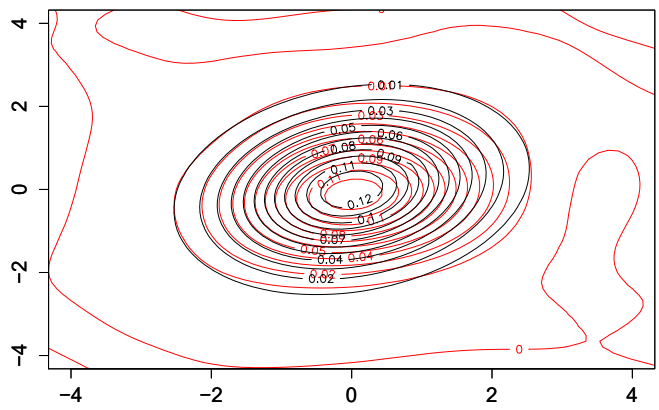

(b) First quartile

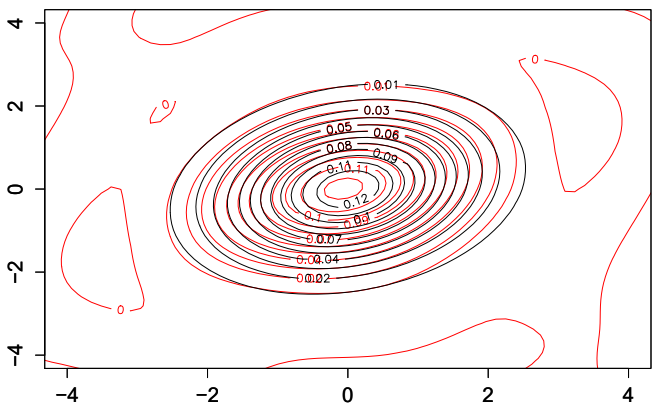

(d) Third quartile

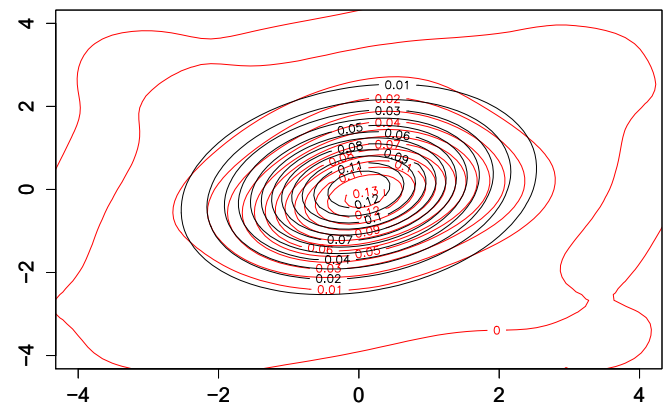

Notes: $V_{1}$ and $V_{2} \sim N(0,1)$. Sample of size $n=1,000$. Target density in black, estimated densities in red: four out of 100 replicated estimates corresponding to the minimum value and the first, second and third quartile of the 100 calculated ISEs. Truncations parameters are $T=U=2.7$. 
Figure 2.10: Contour plots of $\widehat{f}_{X_{1}, X_{2}}$ - normal case $-V_{3} \sim N(0,0.5)$ and $n=100$

(a) Minimum

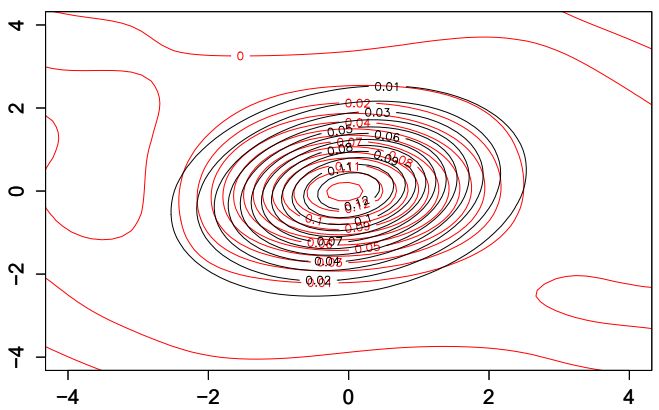

(c) Second quartile

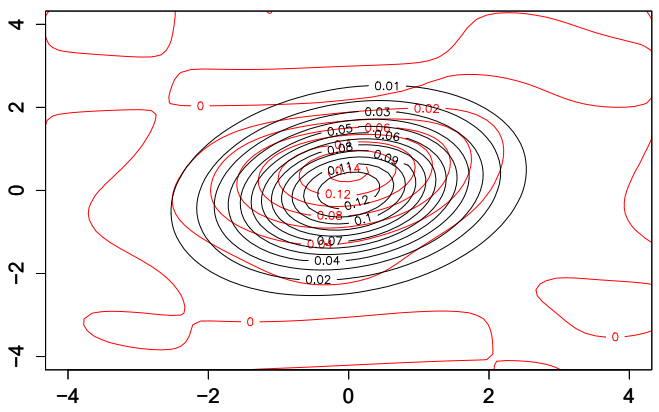

(b) First quartile

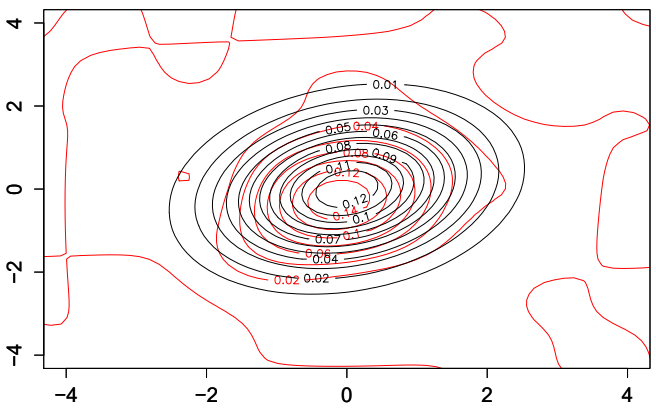

(d) Third quartile

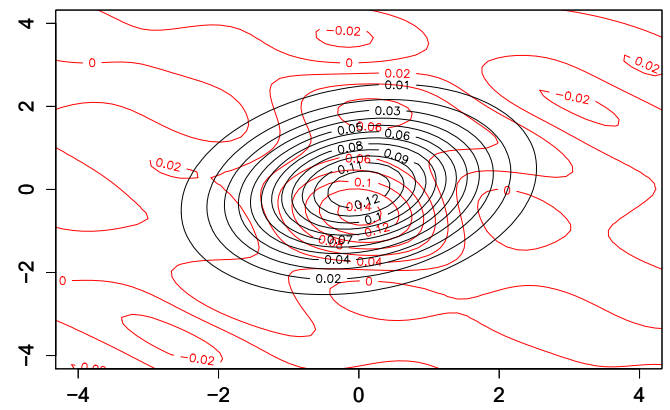

Notes: $V_{1}$ and $V_{2} \sim N(0,1)$. Sample of size $n=100$. Target density in black, estimated densities in red: four out of 100 replicated estimates corresponding to the minimum value and the first, second and third quartile of the 100 calculated ISEs. Truncations parameters are $T=U=2.5$. 
Figure 2.11: 3D plot of $\widehat{f}_{X_{1}, X_{2}}$ - normal case $-V_{3} \sim N(0,0.1)$

(a) Estimated density

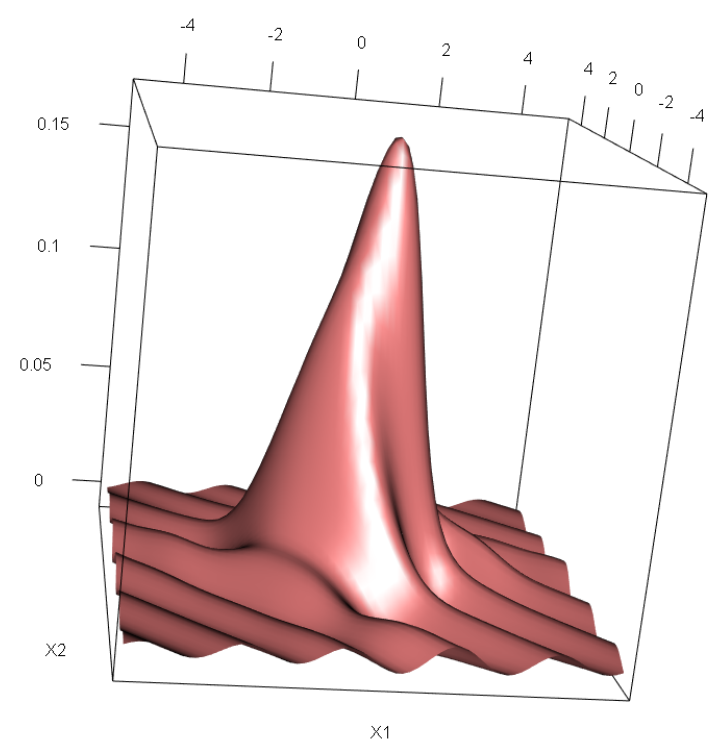

(b) Target density

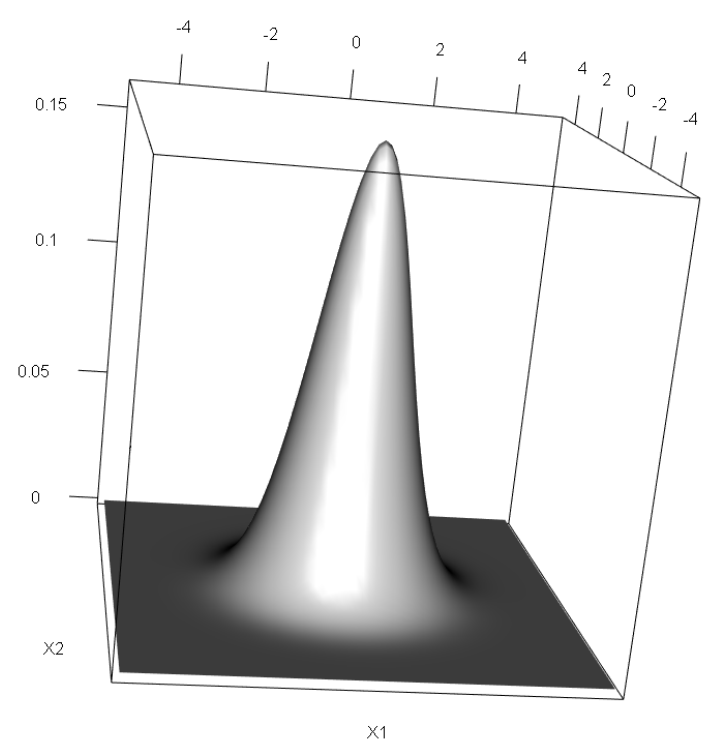

Notes: $V_{1}$ and $V_{2} \sim N(0,1)$. Sample of size $n=1,000$. The estimated densities pictured corresponds to the mean value of the 100 calculated ISEs. 
Figure 2.12: 3D plot of $\widehat{f}_{X_{1}, X_{2}}$ - normal case $-V_{3} \sim N(0,0.5)$

(a) Estimated density

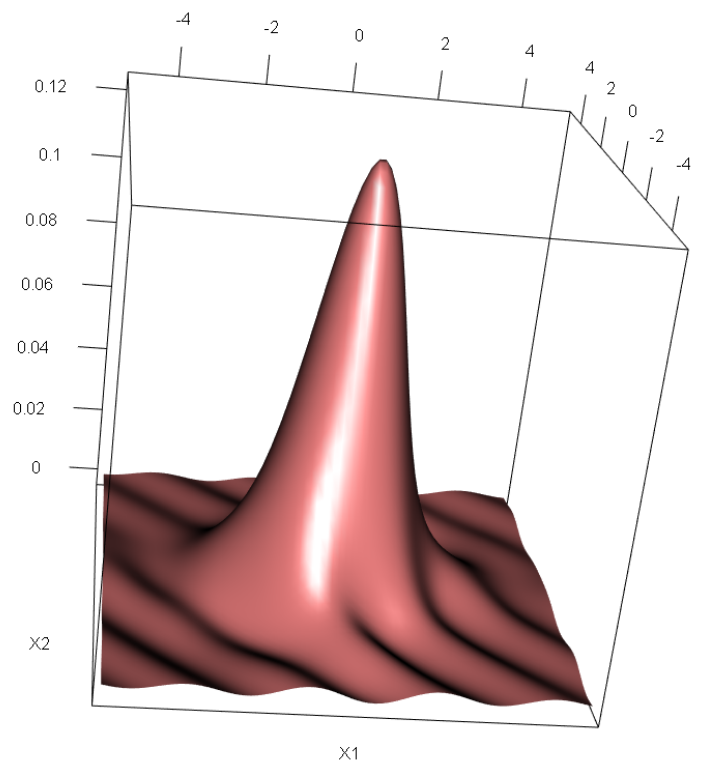

(b) Target density

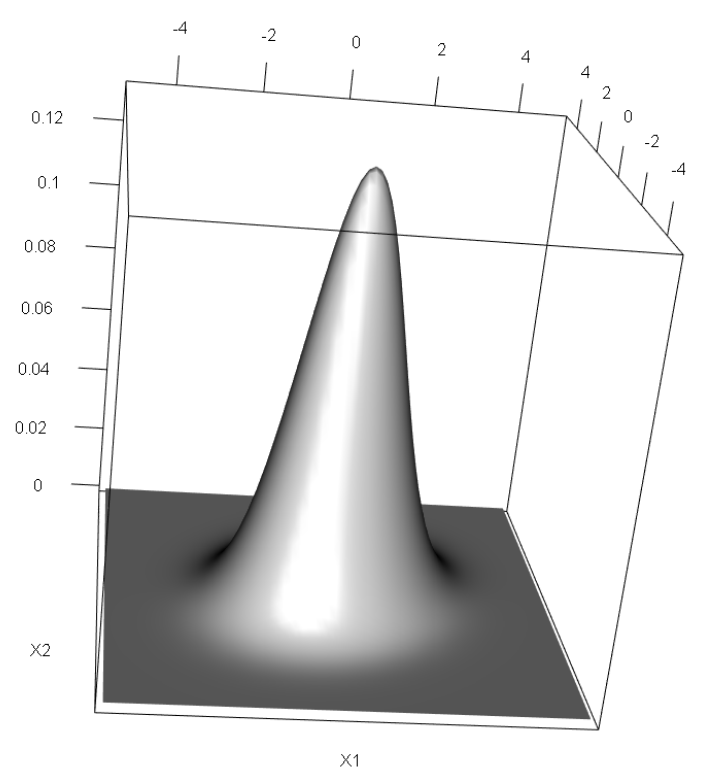

Notes: $V_{1}$ and $V_{2} \sim N(0,1)$. Sample of size $n=1,000$. The estimated densities pictured corresponds to the mean value of the 100 calculated ISEs. 


\section{Appendix C}

\section{Appendix to Chapter 2}

\section{C.1 Proofs of Lemma 1 and Theorem 1}

\section{Proof of Lemma 1:}

This proof follows very closely that of Kotlarski in his seminal paper, also reported in Rao (1992). Explicit formulae for the CFs of $V_{1}, V_{2}$ and $V_{3}$ can also be derived following Rao (1992, Remark 2.1.11). I'll make use of the CFs of $X_{1}, X_{2}$ and $X_{s}$ and their expressions given in Equations (2.8) to (2.10). Note that the condition of non-vanishing $\phi_{X_{1}}(t), \phi_{X_{2}}(t)$ and $\phi_{X_{s}}(t)$ is equivalent to non-vanishing of any of the functions $\phi_{V_{1}}(t), \phi_{V_{2}}(t)$ and $\phi_{V_{3}}(t)$.

Let $U_{1}, U_{2}$ and $U_{3}$ be another three independent real random variables with nonvanishing CFs $\phi_{U_{k}}(t), k=1,2,3$. Also, let $W_{1}=U_{1}+U_{3}, W_{2}=U_{2}+U_{3}$ and $W_{s}=W_{1}+W_{2}$.

Finally, let us define

$$
\gamma_{k}(t)=\phi_{U_{k}}(t) / \phi_{V_{k}}(t), \quad k=1,2,3
$$

Let $X_{1}$ and $W_{1}, X_{2}$ and $W_{2}, X_{s}$ and $W_{s}$ have the same distributions, so that their 
CFs are equal. Hence

$$
\begin{aligned}
\phi_{V_{1}}(t) \phi_{V_{3}}(t) & =\phi_{U_{1}}(t) \phi_{U_{3}}(t), \\
\phi_{V_{2}}(t) \phi_{V_{3}}(t) & =\phi_{U_{2}}(t) \phi_{U_{3}}(t), \\
\phi_{V_{1}}(t) \phi_{V_{2}}(t) \phi_{V_{3}}(2 t) & =\phi_{U_{1}}(t) \phi_{U_{2}}(t) \phi_{U_{3}}(2 t),
\end{aligned}
$$

hold for $-\infty<t<\infty$.

Some manipulations give

$$
\begin{aligned}
\gamma_{1}(t) \gamma_{3}(t) & =1, \\
\gamma_{2}(t) \gamma_{3}(t) & =1, \\
\gamma_{1}(t) \gamma_{2}(t) \gamma_{3}(2 t) & =1,
\end{aligned}
$$

for $-\infty<t<\infty$.

By substitution, it follows that

$$
\gamma_{3}(2 t)=\left[\gamma_{3}(t)\right]^{2}, \quad \text { for }-\infty<t<\infty
$$

Also note that given how they have been defined, we know that $\gamma_{k}(t), k=1,2,3$, are continuous complex-valued functions with $\gamma_{k}(0)=1, k=1,2,3$. Hence the only possible solution for Equation (C.4) is

$$
\gamma_{3}(t)=e^{c t}, \quad \text { for }-\infty<t<\infty
$$

where $c$ is a complex number.

Definition (C.1) implies that $\phi_{U_{3}}(t)=\gamma_{3}(t) \phi_{V_{3}}(t) . \phi_{U_{3}}$ and $\phi_{V_{3}}$ being CFs, they satisfy $\phi .(-t)=\overline{\phi .(t)}$, so that $e^{-c t}=\overline{e^{c t}}$. This further implies that $c$ is purely imaginary and can be written $c=i b$, where $b$ is a real number. 
It follows from Equations (C.3a)-(C.3c) that

$$
\gamma_{3}(t)=1 / \gamma_{1}(t)=1 / \gamma_{2}(t)=e^{i b t}, \quad \text { for }-\infty<t<\infty,
$$

where $b$ is a real constant. This means that $V_{k}$ and $U_{k}$ have the same distributions up to a change of location, for $k=1,2,3$, which proves that the distributions of $X_{1}$, $X_{2}$ and $X_{s}$ determine that of $V_{1}, V_{2}$ and $V_{3}$ up to location only.

\section{Proof of Theorem 1:}

Using the same set-up as in the proof of Lemma 1, it is easy to derive that

$$
\Phi(t, u)=\frac{\phi_{V_{3}}(t+u)}{\phi_{V_{3}}(t) \phi_{V_{3}}(u)}=\frac{\phi_{U_{3}}(t+u)}{\phi_{U_{3}}(t) \phi_{U_{3}}(u)}
$$

for $-\infty<t, u<\infty$. This comes from the fact that the $\gamma_{3}$ functions in the nominator and denominator of $\Phi(t, u)$ cancel out. This proves that the ratio $\Phi(t, u)$ is uniquely determined by the distributions of $X_{1}, X_{2}$ and $X_{s}$. Given Equation (2.11), the CF $\phi_{X_{1}, X_{2}}(t, u)$ is thus also uniquely identified.

\section{C.2 Proof of Theorem 2}

To prove consistency of our estimator we need results on the convergence of the ECF. We use a result from Ridder and $\mathrm{Hu}$ (2012, Lemma 3, p. 370) that gives an almost sure rate of convergence for the ECF without any restriction on the support of the distribution.

Lemma 2 (Hu and Ridder, 2012). Let $\widehat{\phi}(t)=\int_{-\infty}^{\infty} e^{i t x} d F_{n}(x)$ be the ECF of a random sample from a distribution with cdf $F$ and with $E(|x|)<\infty$. For $0<\gamma<\frac{1}{2}$, let 
$T_{n}=o\left(\left(\frac{n}{\log n}\right)^{\gamma}\right)$. Then

$$
\sup _{|t| \leq T_{n}}|\widehat{\phi}(t)-\phi(t)|=o\left(\alpha_{n}\right) \quad \text { a.s. }
$$

with $\alpha_{n}=o(1)$ and $\frac{(\log n / n)^{\frac{1}{2}-\gamma}}{\alpha_{n}}=O(1)$.

Proof. See Ridder and $\mathrm{Hu}$ (2012).

We now proceed to deriving the convergence rate of $\widehat{f}_{X_{1}, X_{2}}$. We have

$$
\begin{aligned}
& \sup \left|\widehat{f}_{X_{1}, X_{2}}\left(x_{1}, x_{2}\right)-f_{X_{1}, X_{2}}\left(x_{1}, x_{2}\right)\right| \\
& \leq \sup \mid \frac{1}{(2 \pi)^{2}} \int_{-T_{n}}^{T_{n}} \int_{-U_{n}}^{U_{n}} e^{-i\left(t x_{1}+u x_{2}\right)}\left(\widehat{\phi}_{X_{1}}(t) \widehat{\phi}_{X_{2}}(u) \frac{\widehat{\phi}_{V_{3}}(t+u)}{\widehat{\phi}_{V_{3}}(t) \widehat{\phi}_{V_{3}}(u)}\right. \\
& \left.-\phi_{X_{1}}(t) \phi_{X_{2}}(u) \frac{\phi_{V_{3}}(t+u)}{\phi_{V_{3}}(t) \phi_{V_{3}}(u)}\right) d t d u \mid \\
& +\sup \left|\int_{|t|>T_{n}} \int_{-U_{n}}^{U_{n}} e^{-i\left(t x_{1}+u x_{2}\right)} \phi_{X_{1}, X_{2}}(t, u) d t d u\right| \\
& +\sup \left|\int_{-T_{n}}^{T_{n}} \int_{|u|>U_{n}} e^{-i\left(t x_{1}+u x_{2}\right)} \phi_{X_{1}, X_{2}}(t, u) d t d u\right| \\
& +\sup \left|\int_{|t|>T_{n}} \int_{|u|>U_{n}} e^{-i\left(t x_{1}+u x_{2}\right)} \phi_{X_{1}, X_{2}}(t, u) d t d u\right|
\end{aligned}
$$

If $\phi_{X_{1}, X_{2}}(t, u)$ is absolutely integrable, the final three terms are $o(1)$. The first term is bounded by ${ }^{1}$

$$
\begin{aligned}
& \frac{1}{(2 \pi)^{2}} \int_{-T_{n}}^{T_{n}} \int_{-U_{n}}^{U_{n}}\left|\widehat{\phi}_{X_{2}}(u) \frac{\widehat{\phi}_{V_{3}}(t+u)}{\widehat{\phi}_{V_{3}}(t) \widehat{\phi}_{V_{3}}(u)}\right|\left|\widehat{\phi}_{X_{1}}(t)-\phi_{X_{1}}(t)\right| d t d u \\
& +\frac{1}{(2 \pi)^{2}} \int_{-T_{n}}^{T_{n}} \int_{-U_{n}}^{U_{n}}\left|\phi_{X_{1}}(t) \frac{\widehat{\phi}_{V_{3}}(t+u)}{\widehat{\phi}_{V_{3}}(t) \widehat{\phi}_{V_{3}}(u)}\right|\left|\widehat{\phi}_{X_{2}}(u)-\phi_{X_{2}}(u)\right| d t d u \\
& \quad+\frac{1}{(2 \pi)^{2}} \int_{-T_{n}}^{T_{n}} \int_{-U_{n}}^{U_{n}}\left|\phi_{X_{1}}(t) \phi_{X_{2}}(u)\right|\left|\frac{\widehat{\phi}_{V_{3}}(t+u)}{\widehat{\phi}_{V_{3}}(t) \widehat{\phi}_{V_{3}}(u)}-\frac{\phi_{V_{3}}(t+u)}{\phi_{V_{3}}(t) \phi_{V_{3}}(u)}\right| d t d u
\end{aligned}
$$

\footnotetext{
${ }^{1}$ Using the identity $\hat{a} \hat{b} \hat{c}-a b c=(\hat{a}-a) \hat{b} \hat{c}+a(\hat{b}-b) \hat{c}+a b(\hat{c}-c)$.
} 
Let $K_{3}(t)=\inf _{|s| \leq t}\left|\phi_{V_{3}}(s)\right|$ and denote $\theta_{n}=K_{3}\left(T_{n}\right)$ and $\vartheta_{n}=K_{3}\left(U_{n}\right){ }^{2}$ Note that, under Assumption 1,

$$
\begin{aligned}
\left|\frac{1}{\widehat{\phi}_{V_{3}}(t)}\right| & =\frac{1}{\left|\phi_{V_{3}}(t)\left(\frac{\widehat{\phi}_{V_{3}}(t)-\phi_{V_{3}}(t)}{\phi_{V_{3}}(t)}+1\right)\right|} \\
& \leq \frac{1}{\left|\phi_{V_{3}}(t)\right|\left(1-\left|\frac{\widehat{\phi}_{V_{3}}(t)-\phi_{V_{3}}(t)}{\phi_{V_{3}}(t)}\right|\right)} \\
& \leq \frac{1}{\inf _{|t| \leq T_{n}}\left|\phi_{V_{3}}(t)\right|\left(1-\frac{\sup _{|t| \leq T_{n}}\left|\widehat{\phi}_{V_{3}}(t)-\phi_{V_{3}}(t)\right|}{\inf _{|t| \leq T_{n}}\left|\phi_{V_{3}}(t)\right|}\right)} \\
& =\frac{1}{\theta_{n}\left(1-o\left(\frac{\beta_{n}}{\theta_{n}}\right)\right)}
\end{aligned}
$$

for $|t| \leq T_{n}$ and almost surely. Similarly, for $|u| \leq U_{n}$,

$$
\left|\frac{1}{\widehat{\phi}_{V_{3}}(u)}\right| \leq \frac{1}{\vartheta_{n}\left(1-o\left(\frac{\beta_{n}}{\vartheta_{n}}\right)\right)} \text { a.s. }
$$

For the first term of (C.10), by Lemma 2 we have

$$
\begin{aligned}
& \frac{1}{(2 \pi)^{2}} \int_{-T_{n}}^{T_{n}} \int_{-U_{n}}^{U_{n}}\left|\widehat{\phi}_{X_{2}}(u) \frac{\widehat{\phi}_{V_{3}}(t+u)}{\widehat{\phi}_{V_{3}}(t) \widehat{\phi}_{V_{3}}(u)}\right|\left|\widehat{\phi}_{X_{1}}(t)-\phi_{X_{1}}(t)\right| d t d u \\
& \leq C T_{n} U_{n} \frac{\sup _{|t| \leq T_{n}}\left|\widehat{\phi}_{X_{1}}(t)-\phi_{X_{1}}(t)\right|}{\theta_{n}\left(1-o\left(\frac{\beta_{n}}{\theta_{n}}\right)\right) \vartheta_{n}\left(1-o\left(\frac{\beta_{n}}{\vartheta_{n}}\right)\right)} \\
& =o\left(\frac{\alpha_{n} T_{n} U_{n}}{\theta_{n} \vartheta_{n}}\right) \quad \text { a.s. }
\end{aligned}
$$

Analogously, the second term of (C.10) can be shown to be a.s. bounded by $o\left(\frac{\alpha_{n} T_{n} U_{n}}{\theta_{n} \vartheta_{n}}\right)$.

\footnotetext{
${ }^{2}$ Note that if $\phi_{V_{3}}(t) \neq 0$ for all $t$, then continuity of $\phi_{V_{3}}(t)$ implies that $K_{3}(t)>0$ for all $t$.
} 
Finally, consider the third term of (C.10). Note that

$$
\begin{aligned}
& \frac{1}{(2 \pi)^{2}} \int_{-T_{n}}^{T_{n}} \int_{-U_{n}}^{U_{n}}\left|\phi_{X_{1}}(t) \phi_{X_{2}}(u)\right|\left|\frac{\widehat{\phi}_{V_{3}}(t+u)}{\widehat{\phi}_{V_{3}}(t) \widehat{\phi}_{V_{3}}(u)}-\frac{\phi_{V_{3}}(t+u)}{\phi_{V_{3}}(t) \phi_{V_{3}}(u)}\right| d t d u \\
& \leq \frac{1}{(2 \pi)^{2}} \int_{-T_{n}}^{T_{n}} \int_{-U_{n}}^{U_{n}}\left|\phi_{X_{1}}(t) \phi_{X_{2}}(u)\right|\left|\frac{\widehat{\phi}_{V_{3}}(t+u)-\phi_{V_{3}}(t+u)}{\widehat{\phi}_{V_{3}}(t) \widehat{\phi}_{V_{3}}(u)}\right| d t d u \\
& \quad+\frac{1}{(2 \pi)^{2}} \int_{-T_{n}}^{T_{n}} \int_{-U_{n}}^{U_{n}}\left|\phi_{X_{1}}(t) \phi_{X_{2}}(u)\right|\left|\frac{\phi_{V_{3}}(t+u)}{\widehat{\phi}_{V_{3}}(t) \widehat{\phi}_{V_{3}}(u)}-\frac{\phi_{V_{3}}(t+u)}{\phi_{V_{3}}(t) \phi_{V_{3}}(u)}\right| d t d u
\end{aligned}
$$

For the integrand in the first term on the right-hand side of (C.14), under Assumption 1 we have

$$
\left|\frac{\widehat{\phi}_{V_{3}}(t+u)-\phi_{V_{3}}(t+u)}{\widehat{\phi}_{V_{3}}(t) \widehat{\phi}_{V_{3}}(u)}\right| \leq \frac{o\left(\beta_{n}\right)}{\theta_{n} \vartheta_{n}\left(1-o\left(\frac{\beta_{n}}{\theta_{n}}\right)\right)\left(1-o\left(\frac{\beta_{n}}{\vartheta_{n}}\right)\right)} \quad \text { a.s. }
$$

Using the same line of proof as in (C.11), for the integrand in the second term on the right-hand side of (C.14) we have

$$
\begin{aligned}
& \left|\frac{\phi_{V_{3}}(t+u)}{\widehat{\phi}_{V_{3}}(t) \widehat{\phi}_{V_{3}}(u)}-\frac{\phi_{V_{3}}(t+u)}{\phi_{V_{3}}(t) \phi_{V_{3}}(u)}\right| \\
& \leq\left|\frac{\phi_{V_{3}}(t+u)}{\widehat{\phi}_{V_{3}}(t) \widehat{\phi}_{V_{3}}(u)}\right|+\left|\frac{\phi_{V_{3}}(t+u)}{\phi_{V_{3}}(t) \phi_{V_{3}}(u)}\right| \\
& \leq \frac{\left|\phi_{V_{3}}(t+u)\right|}{\left|\phi_{V_{3}}(t) \phi_{V_{3}}(u)\right|}\left(\frac{1}{\left(1-\left|\frac{\widehat{\phi}_{V_{3}}(t)-\phi_{V_{3}}(t)}{\phi_{V_{3}}(t)}\right|\right)\left(1-\left|\frac{\widehat{\phi}_{V_{3}}(u)-\phi_{V_{3}}(u)}{\phi_{V_{3}}(u)}\right|\right)}+1\right) \\
& \leq\left|\frac{\phi_{V_{3}}(t+u)}{\phi_{V_{3}}(t) \phi_{V_{3}}(u)}\right|\left(\frac{1}{\left(1-o\left(\frac{\beta_{n}}{\theta_{n}}\right)\right)\left(1-o\left(\frac{\beta_{n}}{\vartheta_{n}}\right)\right)}+1\right) \text { a.s. }
\end{aligned}
$$


Therefore we obtain for the second term on the right-hand side of (C.14)

$$
\begin{aligned}
& \frac{1}{(2 \pi)^{2}} \int_{-T_{n}}^{T_{n}} \int_{-U_{n}}^{U_{n}}\left|\phi_{X_{1}}(t) \phi_{X_{2}}(u)\right|\left|\left(\frac{\phi_{V_{3}}(t+u)}{\widehat{\phi}_{V_{3}}(t) \widehat{\phi}_{V_{3}}(u)}-\frac{\phi_{V_{3}}(t+u)}{\phi_{V_{3}}(t) \phi_{V_{3}}(u)}\right)\right| \\
& \leq \frac{1}{(2 \pi)^{2}} \int_{-T_{n}}^{T_{n}} \int_{-U_{n}}^{U_{n}}\left|\phi_{X_{1}}(t) \phi_{X_{2}}(u) \frac{\phi_{V_{3}}(t+u)}{\phi_{V_{3}}(t) \phi_{V_{3}}(u)}\right|\left(\frac{1}{\left(1-o\left(\frac{\beta_{n}}{\theta_{n}}\right)\right)\left(1-o\left(\frac{\beta_{n}}{\vartheta_{n}}\right)\right)}+1\right) \\
& =\frac{1}{(2 \pi)^{2}} \int_{-T_{n}}^{T_{n}} \int_{-U_{n}}^{U_{n}}\left|\phi_{X_{1}, X_{2}}(t, u)\right|\left(\frac{1}{\left(1-o\left(\frac{\beta_{n}}{\theta_{n}}\right)\right)\left(1-o\left(\frac{\beta_{n}}{\vartheta_{n}}\right)\right)}+1\right)
\end{aligned}
$$

By dominated convergence if $\phi_{X_{1}, X_{2}}(t, u)$ is absolutely integrable and if $\beta_{n} / \theta_{n}=o(1)$ and $\beta_{n} / \vartheta_{n}=o(1)$, then this last term is $o(1)$ almost surely. Finally, from (C.15), the last term on the right-hand side of (C.10) is a.s. of order $\frac{o\left(\beta_{n}\right) T_{n} U_{n}}{\theta_{n} \vartheta_{n}\left(1-o\left(\beta_{n} / \theta_{n}\right)\right)\left(1-o\left(\beta_{n} / \vartheta_{n}\right)\right)}=$ $o\left(\frac{\beta_{n} T_{n} U_{n}}{\theta_{n} \vartheta_{n}}\right)$. Hence we have proved that $\sup \left|\widehat{f}_{X_{1}, X_{2}}\left(x_{1}, x_{2}\right)-f_{X_{1}, X_{2}}\left(x_{1}, x_{2}\right)\right|$ is

$$
o\left(\frac{\alpha_{n} T_{n} U_{n}}{\theta_{n} \vartheta_{n}}\right)+o\left(\frac{\beta_{n} T_{n} U_{n}}{\theta_{n} \vartheta_{n}}\right)
$$

almost surely. 


\section{C.3 Selection Procedure for the Truncation Pa- rameters $U$ and $T$}

By the Fourier inversion, we have that

$$
\begin{aligned}
& \widehat{f}_{X_{1}, X_{2}}\left(x_{1}, x_{2}\right)-f_{X_{1}, X_{2}}\left(x_{1}, x_{2}\right) \\
& =\frac{1}{(2 \pi)^{2}} \iint e^{-i\left(t x_{1}+u x_{2}\right)}\left\{\left(\widehat{\phi}_{X_{1}, X_{2}}(t, u)-\phi_{X_{1}, X_{2}}(t, u)\right) I(|t| \leq T,|u| \leq U)\right. \\
& -\phi_{X_{1}, X_{2}}(t, u)(I(|t|>T,|u| \leq U)+I(|t| \leq T,|u|>U) \\
& +I(|t|>T,|u|>U))\} d t d u
\end{aligned}
$$

where $I(.,$.$) denotes a bi-dimensional indicator function taking the value one if the$ two statements in parentheses are true, and zero otherwise. To ease notation, denote $I_{\text {in }}(t, u ; T, U)=I(|t| \leq T,|u| \leq U)$ and $I_{\text {out }}(t, u ; T, U)=I(|t|>T,|u| \leq U)+$ $I(|t| \leq T,|u|>U)+I(|t|>T,|u|>U)$. Then,

$$
\begin{aligned}
& \iint\left|\widehat{f}_{X_{1}, X_{2}}\left(x_{1}, x_{2}\right)-f_{X_{1}, X_{2}}\left(x_{1}, x_{2}\right)\right|^{2} d x_{1} d x_{2} \\
& =\iint\left|\frac{1}{(2 \pi)^{2}} \iint e^{-i\left(t x_{1}+u x_{2}\right)}\left(\widehat{\phi}_{X_{1}, X_{2}}(t, u)-\phi_{X_{1}, X_{2}}(t, u)\right) I_{\text {in }}(t, u ; T, U) d t d u\right|^{2} d x_{1} d x_{2} \\
& +\iint\left|\frac{1}{(2 \pi)^{2}} \iint e^{-i\left(t x_{1}+u x_{2}\right)} \phi_{X_{1}, X_{2}}(t, u) I_{\text {out }}(t, u ; T, U) d t d u\right|^{2} d x_{1} d x_{2} \\
& =\frac{1}{(2 \pi)^{2}} \iint\left|\widehat{\phi}_{X_{1}, X_{2}}(t, u)-\phi_{X_{1}, X_{2}}(t, u)\right|^{2} I_{\text {in }}(t, u ; T, U) d t d u \\
& +\frac{1}{(2 \pi)^{2}} \iint\left|\phi_{X_{1}, X_{2}}(t, u)\right|^{2} I_{\text {out }}(t, u ; T, U) d t d u,
\end{aligned}
$$


where the last expression is obtained using the Parseval-Plancherel identity. ${ }^{3}$ I now develop and simplify the first part of expression (C.19). In what follows let $\Delta_{X_{k}}=$ $\widehat{\phi}_{X_{k}}-\phi_{X_{k}}$, for $k \in\{1,2, s\}$ and $\Delta_{V_{3}}=\widehat{\phi}_{V_{3}}-\phi_{V_{3}}$. It follows from Equation (2.11) of Section 2.3 that

$$
\begin{aligned}
\widehat{\phi}_{X_{1}, X_{2}}(t, u)-\phi_{X_{1}, X_{2}}(t, u) \\
=\widehat{\phi}_{X_{1}}(t) \widehat{\phi}_{X_{2}}(u) \frac{\widehat{\phi}_{V_{3}}(t+u)}{\widehat{\phi}_{V_{3}}(t) \widehat{\phi}_{V_{3}}(u)}-\phi_{X_{1}}(t) \phi_{X_{2}}(u) \frac{\phi_{V_{3}}(t+u)}{\phi_{V_{3}}(t) \phi_{V_{3}}(u)} \\
=\frac{1}{\phi_{V_{3}}(t) \phi_{V_{3}}(u)}\left(\frac{\widehat{\phi}_{X_{1}}(t) \widehat{\phi}_{X_{2}}(u) \widehat{\phi}_{V_{3}}(t+u)}{\widehat{\phi}_{V_{3}}(t) \widehat{\phi}_{V_{3}}(u) / \phi_{V_{3}}(t) \phi_{V_{3}}(u)}-\phi_{X_{1}}(t) \phi_{X_{2}}(u) \phi_{V_{3}}(t+u)\right) \\
=\frac{1}{\phi_{V_{3}}(t) \phi_{V_{3}}(u)}\left(\frac{\widehat{\phi}_{X_{1}}(t) \widehat{\phi}_{X_{2}}(u) \widehat{\phi}_{V_{3}}(t+u)}{1+\nabla_{3}(t, u)}-\phi_{X_{1}}(t) \phi_{X_{2}}(u) \phi_{V_{3}}(t+u)\right),
\end{aligned}
$$

where $\nabla_{3}(t, u)=\frac{\Delta_{V_{3}}(t) \Delta_{V_{3}}(u)}{\phi_{V_{3}}(t) \phi_{V_{3}}(u)}+\frac{\Delta_{V_{3}}(t)}{\phi_{V_{3}}(t)}+\frac{\Delta_{V_{3}}(u)}{\phi_{V_{3}}(u)}$. Further developing the last expression,

$$
\begin{aligned}
& \widehat{\phi}_{X_{1}, X_{2}}(t, u)-\phi_{X_{1}, X_{2}}(t, u) \\
& \quad=\frac{1}{\phi_{V_{3}}(t) \phi_{V_{3}}(u)}\left(\left(\phi_{X_{1}}(t)-\Delta_{X_{1}}(t)\right)\left(\phi_{X_{2}}(u)-\Delta_{X_{2}}(u)\right)\left(\phi_{V_{3}}(t+u)-\Delta_{V_{3}}(t+u)\right)\right. \\
& \left.\quad \times\left(1+\nabla_{3}(t, u)\right)^{-1}-\phi_{X_{1}}(t) \phi_{X_{2}}(u) \phi_{V_{3}}(t+u)\right) \\
& \quad \approx \frac{1}{\phi_{V_{3}}(t) \phi_{V_{3}}(u)}\left(\Delta_{X_{1}}(t) \phi_{X_{2}}(u) \phi_{V_{3}}(t+u)+\phi_{X_{1}}(t) \Delta_{X_{2}}(u) \phi_{V_{3}}(t+u)\right. \\
& \left.\quad+\phi_{X_{1}}(t) \phi_{X_{2}}(u) \Delta_{V_{3}}(t+u)-\phi_{X_{1}, X_{2}}(t, u)\left(\Delta_{V_{3}}(t) \phi_{V_{3}}(u)+\phi_{V_{3}}(t) \Delta_{V_{3}}(u)\right)\right)
\end{aligned}
$$

where the approximation is obtained by ignoring smaller size terms after linearization by Taylor expansion.

Note that the random variables $\Delta_{X_{1}}$ and $\Delta_{X_{2}}$ are independent with zero means.

\footnotetext{
${ }^{3}$ In the univariate case, the Parseval-Plancherel identity is formulated as follows (Ushakov, 1999, Thm.1.2.10): For an absolutely continuous distribution with density function $f(x)$ and characteristic function $\phi(t),|\phi(t)|^{2}$ is integrable if and only if $f^{2}(x)$ is integrable, and in this case,

$$
\int f^{2}(x) d x=\frac{1}{(2 \pi)} \int|\phi(t)|^{2} d t
$$
}


I assume from now on that all the variables are symmetrically distributed, hence all CFs are real. ${ }^{4}$ By further assuming that $\Delta_{V_{3}}$ is independent of both $\Delta_{X_{1}}$ and $\Delta_{X_{2}}, \mathrm{I}$ obtain: ${ }^{5}$

$$
\begin{aligned}
\mathrm{E}\left|\widehat{\phi}_{X_{1}, X_{2}}(t, u)-\phi_{X_{1}, X_{2}}(t, u)\right|^{2} \\
\quad=\frac{1}{\left|\phi_{V_{3}}(t) \phi_{V_{3}}(u)\right|^{2}}\left\{\mathrm{E}\left|\Delta_{X_{1}}(t) \phi_{X_{2}}(u) \phi_{V_{3}}(t+u)\right|^{2}\right. \\
\quad+\mathrm{E}\left|\phi_{X_{1}}(t) \Delta_{X_{2}}(u) \phi_{V_{3}}(t+u)\right|^{2}+\mathrm{E}\left|\phi_{X_{1}}(t) \phi_{X_{2}}(u) \Delta_{V_{3}}(t+u)\right|^{2} \\
-2 \phi_{X_{1}, X_{2}}(t, u) \phi_{X_{1}}(t) \phi_{X_{2}}(u) \mathrm{E}\left\{\Delta_{V_{3}}(t+u)\left(\Delta_{V_{3}}(t) \phi_{V_{3}}(u)+\phi_{V_{3}}(t) \Delta_{V_{3}}(u)\right)\right\} \\
\left.+\left|\phi_{X_{1}, X_{2}}(t, u)\right|^{2} \mathrm{E}\left|\Delta_{V_{3}}(t) \phi_{V_{3}}(u)+\phi_{V_{3}}(t) \Delta_{V_{3}}(u)\right|^{2}\right\} \\
\quad=\left|\phi_{X_{1}, X_{2}}(t, u)\right|^{2}\left\{\frac{\mathrm{E}\left|\Delta_{X_{1}}(t)\right|^{2}}{\left|\phi_{X_{1}}(t)\right|^{2}}+\frac{\mathrm{E}\left|\Delta_{X_{2}}(u)\right|^{2}}{\left|\phi_{X_{2}}(u)\right|^{2}}+\frac{\mathrm{E}\left|\Delta_{V_{3}}(t+u)\right|^{2}}{\left|\phi_{V_{3}}(t+u)\right|^{2}}\right. \\
\quad-2\left(\frac{\mathrm{E}\left\{\Delta_{V_{3}}(t+u) \Delta_{V_{3}}(t)\right\}}{\phi_{V_{3}}(t+u) \phi_{V_{3}}(t)}+\frac{\mathrm{E}\left\{\Delta_{V_{3}}(t+u) \Delta_{V_{3}}(u)\right\}}{\phi_{V_{3}}(t+u) \phi_{V_{3}}(u)}\right) \\
\left.\quad+\frac{\mathrm{E}\left|\Delta_{V_{3}}(t)\right|^{2}}{\left|\phi_{V_{3}}(t)\right|^{2}}+\frac{\mathrm{E}\left|\Delta_{V_{3}}(u)\right|^{2}}{\left|\phi_{V_{3}}(u)\right|^{2}}+2 \frac{\mathrm{E}\left\{\Delta_{V_{3}}(t) \Delta_{V_{3}}(u)\right\}}{\phi_{V_{3}}(t) \phi_{V_{3}}(u)}\right\}
\end{aligned}
$$

\footnotetext{
${ }^{4}$ This assumption is made for convenience. It helps avoiding too complicated expressions since, for two complex numbers $z_{1}$ and $z_{2},\left|z_{1}+z_{2}\right|^{2}=\left|z_{1}\right|^{2}+\left|z_{2}\right|^{2}+2 \operatorname{Re} z_{1} \operatorname{Re} z_{2}+2 \operatorname{Im} z_{1} \operatorname{Im} z_{2}$, while if $z_{1}$ and $z_{2}$ are real then $\left|z_{1}+z_{2}\right|^{2}=\left(z_{1}+z_{2}\right)^{2}$. Future work may relax this assumption.

${ }^{5}$ This simplifying assumption can also be relaxed, which leads to slightly more complicated mathematical developments and final expression for the asymptotic MISE of $\hat{f}_{X_{1}, X_{2}}$.
} 
Let's look now at the asymptotic behavior of the $\mathrm{E}\left|\Delta_{X_{k}}(t)\right|^{2}$ terms, for $k \in\{1,2, S\}$.

$$
\begin{aligned}
\mathrm{E}\left|\Delta_{X_{k}}(t)\right|^{2} & =\mathrm{E}\left|\widehat{\phi}_{X_{k}}(t)-\phi_{X_{k}}(t)\right|^{2} \\
& =\mathrm{E}\left|\frac{1}{n_{k}} \sum_{j=1}^{n_{k}} e^{i t X_{k j}}-\phi_{X_{k}}(t)\right|^{2} \\
& =\mathrm{E}\left|\frac{1}{n_{k}} \sum_{j=1}^{n_{k}}\left(e^{i t X_{k j}}-\phi_{X_{k}}(t)\right)\right|^{2} \\
& =\frac{1}{n_{k}^{2}} \mathrm{E} \sum_{j=1}^{n_{k}}\left|e^{i t X_{k j}}-\phi_{X_{k}}(t)\right|^{2} \\
& =\frac{1}{n_{k}^{2}} \sum_{j=1}^{n_{k}} \mathrm{E}\left|e^{i t X_{k j}}-\phi_{X_{k}}(t)\right|^{2} \\
& =\frac{1}{n_{k}} \beta_{X_{k}}(t)
\end{aligned}
$$

where $\beta_{X_{k}}(t)=\mathrm{E}\left|e^{i t X_{k j}}-\phi_{X_{k}}(t)\right|^{2}$. As $t \rightarrow \infty, \phi_{X_{k}}(t) \rightarrow 0$, hence $\beta_{X_{k}}(t) \rightarrow$ $\mathrm{E}\left|e^{i t X_{k j}}\right|^{2}=1$. Therefore,

$$
\lim _{t \rightarrow \infty} \mathrm{E}\left|\Delta_{X_{k}}(t)\right|^{2}=n_{k}^{-1}
$$

In order to use result (C.21) in Equation (C.20) in order to approximate the asymptotic MISE of $\widehat{f}_{X_{1}, X_{2}}$, I further express $\Delta_{V_{3}}$ in terms of $\Delta_{X_{1}}, \Delta_{X_{2}}$ and $\Delta_{X_{S}}$. With $\kappa_{3}(t)=\ln \phi_{V_{3}}(t)$, we have

$$
\begin{aligned}
\Delta_{V_{3}}(t) & =\phi_{V_{3}}(t)\left(\widehat{\phi}_{V_{3}}(t) / \phi_{V_{3}}(t)-1\right) \\
& =\phi_{V_{3}}(t)\left(e^{\widehat{\kappa}_{3}(t)-\kappa_{3}(t)}-1\right) \\
& \approx \phi_{V_{3}}(t)\left(\widehat{\kappa}_{3}(t)-\kappa_{3}(t)\right)
\end{aligned}
$$

by Taylor expansion, when ignoring smaller size terms.

Using $\widehat{\kappa}_{3}(t)=\sum_{j=2}^{J_{n}} \hat{a}_{j} t^{j}=V_{t}^{\prime} \hat{a}$, the estimator proposed in Equation (2.43) of 
Section 2.5, $\widehat{\kappa}_{3}(t)-\kappa_{3}(t)$ can be decomposed as follows:

$$
\begin{aligned}
\widehat{\kappa}_{3}(t) & -\kappa_{3}(t) \\
& =V_{t}^{\prime} \hat{a}-\kappa_{3}(t) \\
& =V_{t}^{\prime}\left(V^{\prime} V\right)^{-1} V^{\prime} \ln \widehat{\Phi}-\kappa_{3}(t) \\
& =\left\{V_{t}^{\prime}\left(V^{\prime} V\right)^{-1} V^{\prime}(\ln \widehat{\Phi}-\ln \Phi)\right\}+\left\{V_{t}^{\prime}\left(V^{\prime} V\right)^{-1} V^{\prime} \ln \Phi-V_{t}^{\prime} a\right\}+\left\{V_{t}^{\prime} a-\kappa_{3}(t)\right\}
\end{aligned}
$$

The first error component in (C.22) comes from the fact that $\Phi(t, t)=\frac{\phi_{X_{s}}(t)}{\phi_{X_{1}}(t) \phi_{X_{2}}(t)}$ is not observable. Rather, we observe an unbiased estimate $\widehat{\Phi}(t, t)=\frac{\widehat{\phi}_{X_{s}}(t)}{\widehat{\phi}_{X_{1}}(t) \widehat{\phi}_{X_{2}}(t)}$. The second error component is an estimation error stemming from the difference between a parameter estimate and its true value. Finally, the third error component is the sieve approximation error, which can in principle be controlled for by selecting a sufficiently high sieve order. Denote $r(t)=V_{t}^{\prime} a-\kappa_{3}(t)$ and note that the estimation error can be re-written $V_{t}^{\prime}\left(V^{\prime} V\right)^{-1} V^{\prime} \ln \Phi-V_{t}^{\prime} a=V_{t}^{\prime}\left(V^{\prime} V\right)^{-1} V^{\prime}(V a+r)-V_{t}^{\prime} a=V_{t}^{\prime} r$. Ignoring the error terms containing $r$, I obtain

$$
\begin{aligned}
\widehat{\kappa}_{3}(t) & -\kappa_{3}(t)=V_{t}^{\prime}\left(V^{\prime} V\right)^{-1} V^{\prime}(\ln \widehat{\Phi}-\ln \Phi) \\
& =P_{t}^{\prime} \ln (\widehat{\Phi} / \Phi) \\
& =P_{t}^{\prime} \ln \left(\left(\Delta_{X_{S}} / \phi_{X_{S}}+1\right) /\left(\Delta_{X_{1}} / \phi_{X_{1}} \Delta_{X_{2}} / \phi_{X_{2}}+\Delta_{X_{1}} / \phi_{X_{1}}+\Delta_{X_{2}} / \phi_{X_{2}}+1\right)\right) \\
& \approx P_{t}^{\prime}\left(\Delta_{X_{S}} / \phi_{X_{S}}-\Delta_{X_{1}} / \phi_{X_{1}}-\Delta_{X_{2}} / \phi_{X_{2}}\right)
\end{aligned}
$$

by Taylor expansion, with $P_{t}^{\prime}=V_{t}^{\prime}\left(V^{\prime} V\right)^{-1} V^{\prime}$. Let $\nabla_{X}=\Delta_{X_{S}} / \phi_{X_{S}}-\Delta_{X_{1}} / \phi_{X_{1}}-$ $\Delta_{X_{2}} / \phi_{X_{2}}$, so that

$$
\Delta_{V_{3}}(t) \approx \phi_{V_{3}}(t) P_{t}^{\prime} \nabla_{X}
$$


Further,

$$
\begin{aligned}
\left|\Delta_{V_{3}}(t)\right|^{2} & =\left|\phi_{V_{3}}(t)\right|^{2}\left|\sum_{i=1}^{N} P_{t}[i] \nabla_{X}(t[i])\right|^{2} \\
& =\left|\phi_{V_{3}}(t)\right|^{2}\left\{\sum_{i=1}^{N}\left|P_{t}[i]\right|^{2}\left|\nabla_{X}(t[i])\right|^{2}+2 \sum_{i=1}^{N} \sum_{j \neq i} P_{t}[i] P_{t}[j] \nabla_{X}(t[i]) \nabla_{X}(t[j])\right\}
\end{aligned}
$$

Given the independence assumptions across samples, E $\left|\Delta_{V_{3}}(t)\right|^{2}$ simplifies to

$$
\begin{aligned}
& \mathrm{E}\left|\Delta_{V_{3}}(t)\right|^{2} \\
& =\left|\phi_{V_{3}}(t)\right|^{2}\left\{\sum_{i=1}^{N}\left|P_{t}[i]\right|^{2}\left(\frac{\mathrm{E}\left|\Delta_{X_{S}}(t[i])\right|^{2}}{\left|\phi_{X_{S}}(t[i])\right|^{2}}+\frac{\mathrm{E}\left|\Delta_{X_{1}}(t[i])\right|^{2}}{\left|\phi_{X_{1}}(t[i])\right|^{2}}+\frac{\mathrm{E}\left|\Delta_{X_{2}}(t[i])\right|^{2}}{\left|\phi_{X_{2}}(t[i])\right|^{2}}\right)\right. \\
& \quad+2 \sum_{i=1}^{N} \sum_{j \neq i} P_{t}[i] P_{t}[j]\left(\frac{\mathrm{E} \Delta_{X_{S}}(t[i]) \Delta_{X_{S}}(t[j])}{\phi_{X_{S}}\left(t_{i}\right) \phi_{X_{S}}(t[j])}+\frac{\mathrm{E} \Delta_{X_{1}}(t[i]) \Delta_{X_{1}}(t[j])}{\phi_{X_{1}}(t[i]) \phi_{X_{1}}(t[j])}\right. \\
& \left.\left.+\frac{\mathrm{E} \Delta_{X_{2}}(t[i]) \Delta_{X_{2}}(t[j])}{\phi_{X_{2}}(t[i]) \phi_{X_{2}}(t[j])}\right)\right\}
\end{aligned}
$$

Finally, using (C.21) and observing that $\lim _{t_{i}, t_{j} \rightarrow \infty} \mathrm{E}\left\{\Delta_{X_{k}}\left(t_{i}\right) \Delta_{X_{k}}\left(t_{j}\right)\right\}=0$ for $k \in$ $\{1,2, S\}$, I obtain

$$
\begin{aligned}
\lim _{t \rightarrow \infty} \mathrm{E}\left|\Delta_{V_{3}}(t)\right|^{2} & =\left|\phi_{V_{3}}(t)\right|^{2} \sum_{i=1}^{N}\left|P_{t}[i]\right|^{2}\left(\frac{n_{S}^{-1}}{\left|\phi_{X_{S}}(t[i])\right|^{2}}+\frac{n_{1}^{-1}}{\left|\phi_{X_{1}}(t[i])\right|^{2}}+\frac{n_{2}^{-1}}{\left|\phi_{X_{2}}(t[i])\right|^{2}}\right) \\
& =\left|\phi_{V_{3}}(t)\right|^{2} P_{t}^{\circ 2 \prime}\left(\frac{n_{S}^{-1}}{\left|\phi_{X_{S}}\right|^{\circ 2}}+\frac{n_{1}^{-1}}{\left|\phi_{X_{1}}\right|^{\circ 2}}+\frac{n_{2}^{-1}}{\left|\phi_{X_{2}}\right|^{\circ 2}}\right),
\end{aligned}
$$

where $\circ$ denotes the Hadamard (element-wise) power or product.

Similarly, it can be shown that

$$
\lim _{t, u \rightarrow \infty} \mathrm{E}\left\{\Delta_{V_{3}}(t) \Delta_{V_{3}}(u)\right\}=\phi_{V_{3}}(t) \phi_{V_{3}}(u)\left(P_{t} \circ P_{u}\right)^{\prime}\left(\frac{n_{S}^{-1}}{\left|\phi_{X_{S}}\right|^{\circ 2}}+\frac{n_{1}^{-1}}{\left|\phi_{X_{1}}\right|^{\circ 2}}+\frac{n_{2}^{-1}}{\left|\phi_{X_{2}}\right|^{\circ 2}}\right) .
$$


Note that for large $T$ and $U$, the behaviour of the first term on the right-hand side of Equation (C.19)) depends on the properties of the integrand for large $t$ and $u$. By using Equations (C.21), (C.25) and (C.26) and substituting into (C.20), I obtain the following expression for the asymptotic MISE of $\widehat{f}_{X_{1}, X_{2}}$ as $T$ and $U \rightarrow \infty$ :

$$
\begin{aligned}
\text { AMISE } & \left\{\widehat{f}_{X_{1}, X_{2}} ; T, U\right\} \\
& =\frac{1}{(2 \pi)^{2}} \iint\left|\phi_{X_{1}, X_{2}}(t, u)\right|^{2}\left\{\frac{n_{1}^{-1}}{\left|\phi_{X_{1}}(t)\right|^{2}}+\frac{n_{2}^{-1}}{\left|\phi_{X_{2}}(u)\right|^{2}}+\left(\left(P _ { t + u } \circ \left(P_{t+u}-2 P_{t}\right.\right.\right.\right. \\
& \left.\left.\left.\left.-2 P_{u}\right)\right)^{\prime}+\left(P_{t}+P_{u}\right)^{\circ 2 \prime}\right)\left(\frac{n_{S}^{-1}}{\left|\phi_{X_{S}}\right|^{22}}+\frac{n_{1}^{-1}}{\left|\phi_{X_{1}}\right|^{22}}+\frac{n_{2}^{-1}}{\left|\phi_{X_{2}}\right|^{22}}\right)\right\} I_{i n}(t, u ; T, U) d t d u \\
& +\frac{1}{(2 \pi)^{2}} \iint\left|\phi_{X_{1}, X_{2}}(t, u)\right|^{2} I_{\text {out }}(t, u ; T, U) d t d u, \\
& \equiv \frac{1}{(2 \pi)^{2}} \iint Q(t, u) I_{\text {in }}(t, u ; T, U) d t d u \\
+ & \frac{1}{(2 \pi)^{2}} \iint\left|\phi_{X_{1}, X_{2}}(t, u)\right|^{2} I_{\text {out }}(t, u ; T, U) d t d u,
\end{aligned}
$$

The optimal truncation values of $T$ and $U$ are then found by minimizing an estima-

tor of AMISE $\left\{\widehat{f}_{X_{1}, X_{2}} ; T, U\right\}$. They can also equivalently be solved for numerically after differentiating the AMISE with respect to $T$ and $U$ and equate the expressions obtained to zero. For example, in the simple case where the constraint $T=U$ is imposed,

$$
\begin{aligned}
& \frac{d}{d T} \text { AMISE }\left\{\widehat{f}_{X_{1}, X_{2}} ; T, T\right\} \\
& \quad=\frac{1}{2 \pi^{2}}\left\{\int_{-T}^{T} Q(t, T) d t+\int_{-T}^{T} Q(T, u) d u-\int_{-T}^{T} \phi_{X_{1}, X_{2}}(t, T) d t-\int_{-T}^{T} \phi_{X_{1}, X_{2}}(T, u) d u\right\}
\end{aligned}
$$




\section{C.4 Computational Algorithm for the Two- Dimensional Fourier Inversion}

This algorithm is based on the two-dimensional FFT-based computational algorithm developed in Ignatenko (2010). Consider two intervals $\left[a_{1}, b_{1}\right]$ and $\left[a_{2}, b_{2}\right]$ on which all bivariate data points $\left\{x_{1 i}, x_{2 i}\right\}$ lie. The density estimate will be computed on a grid of $[\sqrt{M} \times \sqrt{M}]$ points, at intervals $\delta_{1}=\left(b_{1}-a_{1}\right) / \sqrt{M}$ and $\delta_{2}=\left(b_{2}-a_{2}\right) / \sqrt{M}$. Let

$$
\begin{aligned}
& x_{1 k_{1}}=a_{1}+k_{1} \delta_{1}, \quad \text { for } k_{1}=0,1, \ldots, \sqrt{M}-1, \\
& x_{2 k_{2}}=a_{2}+k_{2} \delta_{2}, \quad \text { for } k_{2}=0,1, \ldots, \sqrt{M}-1,
\end{aligned}
$$

be the grids of points.

To use the fft function to perform the Fourier inversion, we also discretize the support of the joint CF of $X_{1}, X_{2}$ on a grid of $[\sqrt{M} \times \sqrt{M}]$ points:

$$
\begin{gathered}
t_{q_{1}}=c_{1}+q_{1} \delta_{t}, \quad \text { for } q_{1}=0,1, \ldots, \sqrt{M}-1, \\
u_{q_{2}}=c_{2}+q_{2} \delta_{u}, \quad \text { for } q_{2}=0,1, \ldots, \sqrt{M}-1 .
\end{gathered}
$$

Then we use the trapezoidal rule to approximate the double integral defining the joint 
density as follows:

$$
\begin{aligned}
& f_{X_{1}, X_{2}}\left(x_{1 k_{1}}, x_{2 k_{2}}\right) \\
& =\frac{1}{(2 \pi)^{2}} \int_{-\infty}^{\infty} \int_{-\infty}^{\infty} e^{-i\left(t x_{1}+u x_{2}\right)} \phi_{X_{1}, X_{2}}(t, u) d t d u \\
& \approx \frac{1}{(2 \pi)^{2}} \sum_{q_{1}=0}^{\sqrt{M}-1} \sum_{q_{2}=0}^{\sqrt{M}-1} e^{-i\left(t_{q_{1}} x_{1 k_{1}}+u_{q_{2}} x_{2 k_{2}}\right)} \phi_{X_{1}, X_{2}}\left(t_{q_{1}}, u_{q_{2}}\right) \delta_{t} \delta_{u} \\
& =\frac{\delta_{t} \delta_{u}}{(2 \pi)^{2}} \sum_{q_{1}=0}^{\sqrt{M}-1} \sum_{q_{2}=0}^{\sqrt{M}-1} e^{-i\left(t_{q_{1}}\left(a_{1}+k_{1} \delta_{1}\right)+u_{q_{2}}\left(a_{2}+k_{2} \delta_{2}\right)\right)} \phi_{X_{1}, X_{2}}\left(t_{q_{1}}, u_{q_{2}}\right) \\
& =\frac{\delta_{t} \delta_{u}}{(2 \pi)^{2}} e^{-i\left(c_{1} k_{1} \delta_{1}+c_{2} k_{2} \delta_{2}\right)} \sum_{q_{1}=0}^{\sqrt{M}-1} \sum_{q_{2}=0}^{\sqrt{M}-1} e^{-i\left(a_{1} t_{q_{1}}+a_{2} u_{q_{2}}\right)} e^{-i\left(q_{1} k_{1} \delta_{t} \delta_{1}+q_{2} k_{2} \delta_{u} \delta_{2}\right)} \phi_{X_{1}, X_{2}}\left(t_{q_{1}}, u_{q_{2}}\right) \\
& =\frac{\delta_{t} \delta_{u}}{(2 \pi)^{2}} e^{-i\left(c_{1} k_{1} \delta_{1}+c_{2} k_{2} \delta_{2}\right)} \sum_{q_{1}=0}^{\sqrt{M}-1} \sum_{q_{2}=0}^{\sqrt{M}-1} e^{-i\left(a_{1} t_{q_{1}}+a_{2} u_{q_{2}}\right)} e^{-i\left(q_{1} k_{1} \frac{2 \pi}{\sqrt{M}}+q_{2} k_{2} \frac{2 \pi}{\sqrt{M}}\right)} \phi_{X_{1}, X_{2}}\left(t_{q_{1}}, u_{q_{2}}\right)
\end{aligned}
$$

where the last line is obtained by letting $\delta_{t} \delta_{1}=\frac{2 \pi}{\sqrt{M}}$ and $\delta_{u} \delta_{2}=\frac{2 \pi}{\sqrt{M}}$. This last double sum is a Discrete Fourier transform that can be computed using R's fft function. Note that it follows from $\delta_{t} \delta_{1}=\frac{2 \pi}{\sqrt{M}}$ and $\delta_{u} \delta_{2}=\frac{2 \pi}{\sqrt{M}}$ that $\delta_{t}=\frac{2 \pi}{b_{1}-a_{1}}$ and $\delta_{u}=\frac{2 \pi}{b_{2}-a_{2}}$, hence the $t_{q_{1}}$ and $u_{q_{2}}$ grids used, centered on zero, are:

$$
\begin{gathered}
t_{q_{1}}=-\frac{\pi \sqrt{M}}{b_{1}-a_{1}}+q_{1} \frac{2 \pi}{b_{1}-a_{1}}, \quad \text { for } q_{1}=0,1, \ldots, \sqrt{M}-1, \\
u_{q_{2}}=-\frac{\pi \sqrt{M}}{b_{2}-a_{2}}+q_{2} \frac{2 \pi}{b_{2}-a_{2}}, \quad \text { for } q_{2}=0,1, \ldots, \sqrt{M}-1 .
\end{gathered}
$$




\section{Chapter 3}

\section{Canadian Households' Payment}

\section{Habits: 'Partnering Up'}

\section{Aggregated Household Data and Individual Data}

\subsection{Introduction}

In the recent applied literature on payment behaviors, intra-household influences are typically ignored due to a lack of data. This section analyzes intra-household methods of payment usage. I provide evidence of strong interdependencies within couple households with respect to payment and cash management practices. Used separately, aggregated household data or individual-level data (where only one person is surveyed in a given household) do not allow taking this important dimension into account.

Household-level data that only provide aggregate household information overlook intra-household heterogeneity. This can be especially problematic in the context 
of payments where households are not the relevant decision-making units. On the other hand, individual-level data that only provide individual information on the respondent ignores intra-household interdependence. This data limitation imposes restrictive modelling assumptions that can be inaccurate and induce, for example, omitted variable bias.

By combining aggregated household data and individual data using the methodology proposed in Chapter 2, however, joint payment behaviors in non-single households can be recovered. This chapter is organized as follows. In Section 3.2 I present the background of this application and the related empirical literature. The data sets are described in the following section. Section 3.4 proposes an exploratory analysis that motivates both the object of this analysis and the pertinence of the methodological approach. In Section 3.5, the validity of the methodology and the appropriateness of the assumptions for the data at hand are assessed. I also evaluate the performance of the approach in a real data setting using test data. The final estimation results are discussed in Section 3.6.

\subsection{Background}

The Bank of Canada is responsible for issuing Canadian bank notes. The planning of its operation requires to 1) obtain precise estimates of the use of cash by the Canadian population, and 2) understand the potential substitution from cash to other retail payment methods. Surveys are important instruments to monitor the population's payment habits. The last Methods-of-Payment (MOP) survey conducted in 2013 by the Bank of Canada indicates that cash is still widely used, especially for small-value transactions. The market share of cash in terms of the number of retail transactions continues however to decrease, while credit cards and more innovative payment instruments, such as contactless credit cards and stored-value cards, are 
being increasingly used; see Fung et al. (2015).

A recent empirical literature provides evidence of the impact of these payment innovations on cash usage for retail payments. Fung et al. (2014) find that the use of contactless credit and stored-value cards decrease the recourse to cash, particularly in terms of volume. Using panel data, Chen et al. (forthcoming) find a statistically significant reduction in cash use resulting from the use of stored-value cards. These two studies were based respectively on individual-level survey data, where information is measured about only one person in the household, and household survey data, where only household aggregates (i.e., family totals) are observed. These are typically the type of data used in the literature on methods of payment. One major limitation of theirs is that they do not allow for analyzing or controlling for the influence of other household members on methods of payment choices. This is because we do not observe the individual behaviors of multiple persons in a given household, which constitutes a missing data problem.

The literature exploring financial practices of couples provides some arguments as to why it might be misleading to examine couples as a whole at the aggregate level. Pahl $(2005,2008)$ and Vogler et al. (2006), among others, document a general move towards individualization in family finances. This term designates a change in households' financial arrangements toward more individualistic money management practices such as partial pooling - as opposed to complete pooling - of resources. The individualization of couple finances implies that the household cannot be regarded as a single economic unit. There are variations between individuals within households in terms of their spending priorities and responsibilities, or even their standards of living (for example, heterogeneity in terms of access to credit) that might not be detectable in household-level data.

As heterogeneous as they may be, households are composed of interdependent individuals. It might therefore be misleading to examine individuals living in couple 
relationships in isolation from their spouse. Investigating credit cards usage within married couples, Pahl (1999) finds that the employment situations of both the man and the woman are relevant to whether or not people use credit cards. ${ }^{1}$ More generally, in couple households, the partner's choices and characteristics are likely to contribute to explaining an individual's behavior. Using individual-level data (where a single individual is observed in each household) might thus give an incomplete picture of what is at stake in explaining methods of payment adoption and usage.

\subsection{Survey Data on Methods of Payment}

The Bank of Canada collects information on Canadian's payment behavior via two surveys: the Method-of-Payments (MOP) Survey and the Canadian Financial Monitor (CFM). The CFM is a comprehensive survey questionnaire on household finances that includes some sections on payments, while the MOP specifically focuses on payment choices. ${ }^{2}$ The latter consists of a survey questionnaire and a payment diary, in which respondents record all cash and non-cash transactions over a three-day period.

In addition to demographics, the CFM and MOP survey questionnaires both collect information on payment choices and cash management using recall questions. Respondents are invited to assess their past or "typical" behavior in terms of cash withdrawals frequencies and amounts, cash holdings, and payments instruments used for making purchases. Beyond cash and conventional debit and credit cards, both questionnaires collect information on alternative payment methods.

Several retail payment innovations introduced in recent years are reshaping the payments landscape; see Arango et al. (2012). Consumers now have a very wide set

\footnotetext{
${ }^{1}$ This study is noteworthy as one of the few that explores intra-household payment behaviors. It focuses, however, on credit cards.

${ }^{2}$ The CFM is a syndicated survey run by Ipsos Reid since 1999; questions on payment methods and cash management were introduced in collaboration with the Bank of Canada in 2009. The MOP survey was commissioned by the Bank of Canada and conducted in collaboration with Ipsos Reid in 2009 and 2013. Details on the 2009 and 2013 MOP Surveys are available in Arango and Welte (2012) and Henry et al. (2015).
} 
of options for making payments at the point of sale. Contactless credit (CTC) and debit cards using near field communication technology allow consumers to make a payment by simply waving the card in front of a payment terminal ("tap-and-go"). Many merchants now offer their own (single-use) prepaid stored-value cards (SVC) that can serve as gift cards or as a convenient way for their customers to pay for frequent purchases. Multi-purpose stored-value cards, in contrast, are issued by credit card companies such as Visa and MasterCard, and are typically accepted wherever traditional credit cards are accepted. New mobile phone applications are also being developed that allow person-to-person transfers and even payment to certain retailers. Because they mimic some desirable features of cash, such as speed, anonymity or ease of use, these payment innovations tend to compete with cash.

While the CFM and MOP surveys collect very similar information, one critical difference between them regards the unit of observation. Table 3.1 summarizes, for couple households, the data observed in both surveys. In the MOP, the sampling unit and unit of observation is the individual respondent. All the questions relate to the respondent's own individual characteristics and behaviors. In the CFM, the sampling unit and main unit of observation is the household. Cash and payment innovations quantities are reported at the aggregated household level: the respondent is asked whether someone in the household uses each method and, if so, to report the monthly family total (in terms of volume and value). ${ }^{3}$ Still, some disaggregated information is provided in the CFM data. First, demographic characteristics are available for the female and male heads of the household. ${ }^{4}$ Second, regarding financial products such as credit cards, bank accounts and debit cards, the respondent is asked to enumerate all products in the household and indicate who owns them. Finally, specific sections

\footnotetext{
${ }^{3}$ The CFM explicitly asks the "person most knowledgeable about [the] household finances" to complete the survey.

${ }^{4}$ The CFM questionnaire is designed such that each household can have at most one male head and one female head. Due to data availability, I thus adopt in the present application the operational definition of a couple as a man and a woman living in married or cohabiting opposite-sex relationships.
} 
such as cash management (cash handling and cash holdings) collect information about the respondent's own personal behavior.

For the present analysis I exploit the 2013 MOP survey data and the 2013 CFM data. The MOP sample is composed of 3,663 individuals, surveyed in November 2013. In the 2013 CFM sample, 12,280 households are observed between January and December. More precisely, about 1,000 households are surveyed each month, and a given household can participate at most once in each 12-month period. The two samples are considered to be independent of one another. ${ }^{5}$

\subsection{Motivating Exploratory Analysis}

As described in the preceding section, the CFM and MOP survey data do not permit to observe both the male and female heads' individual practices in terms of cash and payment innovations usage. However, the available data allow for conducting some exploratory analysis of intra-household influences and inter-households differences with respect to the choice of methods of payment for retail purchases.

The first objective of the present section is to demonstrate the importance of the spouse's influence for explaining a person's behavior, and show that intra-household interactions with respect to payments should not be ignored. This motivates the estimation and analysis of partners' joint distributions of methods of payment usage. To perform this first exploratory analysis I exploit the fact that, in the CFM data, individual information is collected on all credit cards (CC) and debit cards (DC) owned in the household. This allows assessing, in various ways, partners' influence within couple households. I start by analysing partner's interdependencies with respect to CC and DC usage. I further evaluate how individual cash management practices are

\footnotetext{
${ }^{5}$ In the 2013 MOP, respondents who had recently filled out the CFM survey were partially used for recruitment. This led to an overlap (units in common) between the 2013 CFM sample and the 2013 MOP sample. Although this overlap is ignored for the present analysis, it could for example be exploited to allow for measurement error in some variables; see discussion in Section 2.4.4.
} 
impacted by the own and spouse payment habits in terms of CC and DC use.

The second objective is to discern differences both between households (of different types) and within households with respect to payments. This motivates the relevance of the methodology applied, which allows for heterogeneity across households of different types in addition to intra-household heterogeneity. Using MOP data, I explore whether individual payment behaviors differ across household types (single, couple and other households), or across gender. Both unconditional and conditional analyses are performed.

\subsubsection{Intra-household influences with respect to methods of payment}

\subsubsection{Partner's influence on individual methods of payment usage - the case of $\mathrm{CC}$ and $\mathrm{DC}$}

Using CFM data, I analyse partners' joint probability of using two traditional methods of payment: $\mathrm{CC}$ and DC. In the CFM data, this is possible because individual information is collected about all credit cards and bank accounts owned by members of the household. The respondent must indicate whether each product is owned by the female head, the male head, jointly owned or held by another person in the household (which is not specified). Other information requested include the number of purchases completed from each account. For jointly owned CC and DC, only total purchases are reported - it is unknown how these are split between the male and female head. For that reason, I only consider as individual usage the use of individually owned CC and DC for making purchases.

Observed unconditional joint probabilities of use are given in Table 3.2. For CC, individual usage as observed in the margins of the tables looks quite symmetric across genders. A formal two-sample equality test applied to the proportions does not reject 
the null hypothesis at usual significance levels. 20\% of couple households have two individual users, while $40 \%$ of them have no individual user. The picture is quite different for DC, since a large majority of couple households have two non-users, and only $9 \%$ of them have two individual users. ${ }^{6}$ Also, the proportion of individual DC users is significantly larger among female heads than male heads.

Pearson's chi-squared test for the independence of rows and columns in two-way tables reject the null hypothesis in both the $\mathrm{CC}$ and $\mathrm{DC}$ cases. To better assess intra-couple influence in terms of individual CC and DC usage, Table 3.3 presents the probabilities of female and male heads's use of CC or DC conditional on the partner's behavior. For both types of payment cards, an individual's probability of using is higher if the partner uses. Nonetheless, partner's influence seems to be much more important for DC than for CC.

I further proceed to conditional regression analysis over the CFM subsample of couple households. Bivariate probit regressions of female and male heads' use are estimated separately for CC and DC. Results are presented in Table 3.4. After controlling for observable characteristics such as personal and household demographics, types of expenditures and month of participation to the CFM survey, the coefficients of correlation between the residuals are positive and highly significant. ${ }^{7}$ The male and female heads' probabilities of individual usage seem therefore strongly positively associated (more so in the case of DC).

\footnotetext{
${ }^{6}$ Recall that only individually owned CC and DC are considered in this analysis; jointly owned bank and credit card accounts are ignored.

${ }^{7}$ To avoid potential endogeneity issues, household expenditures are expressed as a ratio relative to the average within the individual's demographic stratum (defined according to age and income group), following Stango (2000). This applies everywhere in the chapter.
} 


\subsubsection{Partner's influence on personal cash management practices - the case of CC and DC}

Further exploiting the specificities of the CFM data, I explore whether the respondent's individual cash management practices are influenced by its partner's CC and DC use. Recall indeed that in the CFM data, information on cash management practices are collected at the respondent's level; cf. Table 3.1. Two cash management variables are considered: cash holdings on hand and total number of cash withdrawals past month. They are modelled using negative binomial count regressions with the following explanatory variables: self and partner's payment card use and their interaction, self and partner's personal demographics, household demographics, expenditure types and month of participation to the CFM survey. Results are reported in Table 3.5. In all cases, the partner's main effect and/or the interaction term is significant.

This analysis thus suggests that, if we cannot observe both spouses behaviors in a couple household, we might be missing part of the big picture and unable to correctly explain an individual's payment practices. In more rigorous terms, regressions using individual-level payment data might suffer from omitted variable bias due to intrahousehold interdependence. This motivates the application of the methodology proposed in the previous chapter to recover joint intra-household payment behaviors from the combination of the CFM and MOP data. On the other hand, aggregate household information might hide important intra-household heterogeneity that should not be overlooked - especially in a context as payments where the main decision-making units are individuals, not households. 


\subsubsection{Inter- and intra-household heterogeneity with respect to methods of payment}

\subsubsection{Comparing distributions across household type and gender}

Using MOP data, I compare payment behaviors of survey respondents living in single and couple households by running two-sample Kolmogorov-Smirnov tests for the equality of distribution functions. Payment behavior is measured by the number of purchases made using five methods of payment: CC, DC, SVC, CTC and cash. Table 3.6 presents tests results obtained on all respondents (irrespective of their gender), and by gender. Differences across single and couple households are observed for the three conventional methods of payment CC, DC, and cash, but not for SVC and CTC.

A similar analysis is carried out to compare male and female heads within couple households in the MOP sample. The empirical cumulative distribution functions by family head gender with their 95\% confidence intervals are displayed in Figure 3.1. Kolmogorov-Smirnov tests results are reported in Table 3.7. Significant differences across heads' behaviours are detected for CC, DC and CTC. ${ }^{8}$

\subsubsection{Regression analysis of household heterogeneity}

Using MOP data, I further investigate inter- and intra-household heterogeneity via conditional analysis. Here, three household types are considered: single households with one adult only, couple households with two adults living in married or cohabiting opposite-sex relationships, and other households. ${ }^{9}$ The following five payment

\footnotetext{
${ }^{8}$ Differences across male and female heads with respect to CC and DC usage are confirmed using CFM data. When considering CC and DC purchases made using individually owned payment cards only, equality of distributions across gender is always rejected (for purchases both in terms of volume and value). Results are not reported.

${ }^{9}$ The presence of children in a household of any type is accounted for via control variables. Note that in this analysis, due to data availability, children below 18 years old are not regarded as payment-making individuals.
} 
methods are again considered separately: CC, DC, SVC, CTC and cash.

I first estimate a person's probability of using a given method, conditional on personal and household demographics, as well as personal perceptions about method of payment attributes. Results of probit regressions run on all MOP households are summarized in Table 3.8. I also model the number of monthly purchases made with each method of payment using negative binomial regressions, and the share of monthly retail purchases made with each method of payment (when the frequency of each method usage is normalized by the total number of retail purchases) using fractional response regressions. Results are presented in Tables 3.9 and 3.10.

For most methods of payment, an individual's probability of use does not seem to be significantly impacted by the family type. One exception is for SVC, which people in non-single households are less likely to use than single persons. However, household type seems to have more explanatory power in explaining the volume of use, both when expressed in terms of count or relative share. In other words, the family type impacts the intensive rather than the extensive margin of payment means usage. To correctly control for scale effects, it is best to express methods of payment usage as ratios to the total number of all purchases. It can be observed in Table 3.10 that singles use cash and close substitutes such as SVC and CTC relatively more than individuals living in non-single households. This result is in line with the findings of Burdett et al. (2016) suggesting that being single is cash intensive, which the authors explain by the different types of expenditures made in different types of households: goods and services associated with monetary exchange - such as dining out - are demanded in greater quantities by single than by married people. Consistently, the coefficient estimates for couple households are positive in the case of CC and DC usage. However, these are not significant at usual significance levels.

In brief, once conditioning on other characteristics, most differences between single and couple households are observed for SVC and cash. For CC and CTC, disparities 
are detected between single and other households only. Gender also significantly influences payment behaviors in directions that differ across payment methods - but are consistent across the three regressions run.

These results point to differences in terms of individual payment behavior both across households of different size, and between partners within couple households. This suggests that estimates obtained using aggregate household data might hide important intra-household heterogeneity. It also indicates that it is in general not appropriate to rely on non-couple households (single or other households) to identify quantities of interest regarding couple households. These conclusions motivate the application of the methodology proposed in Chapter 2 to recover joint intra-household payment behaviors from the CFM and MOP data, as it allows for heterogeneity both across households of different types and within non-single households.

\subsection{Validity Assessment of the Methodological Ap- proach}

The methodology developed in the preceding chapter comes with various assumptions and restrictions. Their appropriateness for the data at hand can to a certain extent be examined, and this is the object of the present section. First, compatibility checking across samples and associated empirical distributions is performed. Compatibility of the data with the restrictions of the main model is then tested. Such ex-ante validity assessment should be systematically implemented before applying the proposed methodology, and the specific model used should be chosen accordingly. In addition, I perform an ex-post performance evaluation by employing a test data set. The latter permits to apply the data combination strategy to variables that resemble the real data to be analyzed in the next section, and to compare its outcome to estimates obtained directly on bivariate samples. 


\subsubsection{Ex-ante compatibility checking}

\subsubsection{Compatibility across samples}

The analysis presented in this chapter relies on the combination of the 2013 MOP sample (subsample of couple households) with the 2013 CFM sample (subsample of couple households), with the concern that the two samples should be representative of the same underlying population. Tables 3.11 and 3.12 describe the CFM and MOP samples. Overall, household demographics profiles align well across samples, both with and without the use of sample weights. When examining individual characteristics of persons living in couple households, some discrepancies across the CFM and MOP samples are observed for both the female and male heads. However, the use of sample weights mitigates most of these differences. ${ }^{10}$

\subsubsection{Compatibility across empirical distributions}

Another issue in estimating a joint density (or a probability mass function) by combining marginal densities (or probability mass functions) is compatibility of the distributions. Until now we have assumed that the individual and aggregate distributions are compatible, in the sense that an underlying joint density exists. Here, I perform a series of simple tests to check necessary conditions for the compatibility of the empirical distributions associated with the MOP and CFM samples.

In what follows, I concentrate on five variables measuring payment behavior: the number of monthly purchases made using CC, DC, SVC, CTC, and cash. Summary statistics for each variable, as measured at the individual level in the MOP data (on the male and female heads subsamples) and at the couple household aggregate level in the CFM data, are presented in Table 3.13. The CFM sample sizes are much

\footnotetext{
${ }^{10}$ This result seems to suggest that sample weights should be used. Kernel density estimation methods and theoretical results for iid data, however, do not directly apply to complex survey data; see Buskirk and Lohr (2005). The current setup assumes iid observations, and incorporation of weights and survey design information is left for future work.
} 
larger than that of the MOP. As expected, the CFM variable means are in general larger than the MOP values. Covariance and correlation coefficients between male and female heads, computed by combining variance estimates for the three samples, are also provided. Correlations are most important for CC and CTC usage.

More formal tests on sample means can be implemented using the information on mean estimates confidence intervals provided in Table 3.14. We observe that in most cases, the 95\% confidence intervals for the sum of female and male mean estimates obtained on the MOP samples do not overlap with the confidence intervals for the mean of household totals obtained on the CFM sample. This result is not too surprising, as the mean is very sensitive to the presence of outliers at the tail of the distribution, and suggest that tests on medians might be more appropriate. A consequence of this observation is that the variables should be appropriately recentered before proceeding to the joint distribution estimation.

Another compatibility test concerning the proportion of zeros in the samples is performed. Recall that the Fréchet inequality provides bounds on the joint probability $\operatorname{Pr}\left(X_{F H}, X_{M H}\right)$ as follows:

$$
\begin{array}{r}
\max \left(0, \operatorname{Pr}\left(X_{F H}=x_{F H}\right)+\operatorname{Pr}\left(X_{M H}=x_{M H}\right)-1\right) \leq \operatorname{Pr}\left(X_{F H}=x_{F H}, X_{M H}=x_{M H}\right) \\
\leq \min \left(\operatorname{Pr}\left(X_{F H}=x_{F H}\right), \operatorname{Pr}\left(X_{M H}=x_{M H}\right)\right)
\end{array}
$$

Table 3.15 presents $95 \%$ confidence intervals for estimates of the proportion of zeros in the various data samples. The test consists in verifying that the joint probability $\operatorname{Pr}\left(X_{F H}=0, X_{M H}=0\right)=\operatorname{Pr}\left(X_{H H}=0\right)$, as measured in the CFM data, lies inside the Fréchet bounds estimates provided by the MOP sample. In two cases out of five (DC and CTC), the CFM estimates are significantly larger than the Fréchet upper bounds. This apparent incompatibility might indicate, among other things, the presence of measurement error issues due for example to seasonality effects. The 
MOP was indeed run in November 2013, while the CFM survey runs all year long. As a matter of fact, when regarding only households that participated to the CFM in November 2013, both incompatibilities disappear.

\subsubsection{Compatibility of empirical distributions with the main model}

In Section 2.4, I provided restrictions implied by Model 1 that are testable in the data. Relations given in Equations (2.13) and (2.14) must necessarily hold in the data in order for this model to be valid. The first condition imposes that the correlation coefficient between $X_{F H}$ and $X_{M H}$ be non-negative. From Table 3.13, it is clear that Model 1 is not valid in the case of CTC monthly purchases, for which another model should be used.

More formal tests comparing the sample variances can be implemented using the information on variance estimates confidence intervals provided in Table 3.16. First, restriction (2.13) can be rewritten $\operatorname{Var}\left(X_{F H}+X_{M H}\right) \geq \operatorname{Var}\left(X_{F H}\right)+\operatorname{Var}\left(X_{M H}\right)$. Estimates of $\operatorname{Var}\left(X_{F H}+X_{M H}\right)=\operatorname{Var}\left(X_{H H}\right)$ are obtained on the CFM data, while estimates of individual quantities' variances are obtained on the MOP data. In all cases except for CTC, the variance of the household total is not significantly smaller than the sum of the variances of the male and female variables. Second, restrictions (2.14) are equivalent to $\operatorname{Var}\left(X_{F H}\right) \geq \operatorname{Cov}\left(X_{F H}, X_{M H}\right)$ and $\operatorname{Var}\left(X_{M H}\right) \geq \operatorname{Cov}\left(X_{F H}, X_{M H}\right)$. These conditions hold for all five variables considered.

\subsubsection{Real data performance evaluation using test data}

Before proceeding to the estimation of the joint distributions of interest, the real data performance of the proposed methodology is evaluated using test data. To do so, I exploit once more the fact that in the CFM data, individual information is collected on all CCs and DCs owned in the household. Individually-owned CCs and DCs are observed for both the male and female heads of a given household. I then sum the 
male and female individual $\mathrm{CC}$ and DC payment variables within each household to obtain household total variables. ${ }^{11}$ Descriptive statistics are provided in Table 3.17. This test data set permits one to apply the proposed data combination strategy, as well as to observe the joint distributions within couples directly on bivariate samples.

Because the real variables to be analysed in the next section are not symmetrically distributed, I cannot readily apply the truncation parameter selection proposed in the preceding chapter. Using test data, however, I can assess the impact of truncation by computing integrated squared errors (ISE) for different values of the truncation parameters $T$ and $U .{ }^{12}$ The ISE is calculated by comparing joint PMFs estimated via data combination to empirical joint PMFs directly observed in the bivariate samples. Results are shown in Figure 3.2. For each method of payment, the solid black line corresponds to ISE estimates obtained on raw data. These are quite high, which is symptomatic of a challenging feature of the data: the (discrete) distributions are highly skewed, with large proportions of observations situated on the single mass point $(0,0)$. The ISEs obtained when the estimation is conditioned on positive values only, with zeros being discarded from all variables, are indeed smaller by two orders of magnitude (dashed grey line in Figure 3.2). In all cases a clear-cut threshold at about $T=U=4$ is evident, beyond which the estimator's performance steeply declines.

The methodological approach proposed in Chapter 2 relies on the identification of the joint $\mathrm{CF}$ of $X_{F H}, X_{M H}$ (female and male heads) using a combination of the CFs of $X_{F H}, X_{M H}$ and $X_{H H}$, where $X_{H H}=X_{F H}+X_{M H}$. However, the ISE results discussed so far depend not only on the validity of this identification condition, but also on the performance of the second-step Fourier inversion of the estimated joint CF. To precisely assess the validity of the identification approach, it is therefore more relevant to compare the PMF estimate obtained by sample combination to the PMF

\footnotetext{
${ }^{11}$ Jointly owned CC and DC are again discarded for this exercise.

${ }^{12}$ All variables considered in this application are discrete with lattice-type distributions, as they take only non-negative integer values. Practical implementation of the Fourier inversion to recover the joint PMF of $X_{F H}, X_{M H}$ in this case was discussed in Section 2.6.4.2.
} 
estimate obtained by inverting the joint ECF of $X_{F H}, X_{M H}$ estimated directly on the bivariate sample available in the test data set. For a given truncation value, discrepancies observed between these two PMF estimates will most likely stem from a failure of the identification strategy, and not from a deficient Fourier inversion procedure. The dotted black lines in Figure 3.2 show very good performance of the identification strategy for truncation parameters below 4 .

The performance of the sample combination approach on real-looking data is now further diagnosed in various ways. In all cases, estimates obtained by application of the proposed sample combination strategy are compared with estimates resulting from more direct estimation methods based on the bivariate test data samples: those obtained directly on the bivariate samples as well as those recovered by inversion of the joint ECF of $X_{F H}, X_{M H}$ as measured on the bivariate samples.

Figures 3.3 and 3.4 compare the marginal distribution functions produced by these three approaches. For brevity purposes, we focus here on the case of DC. Figure 3.3 demonstrates again clearly the difficulty of the proposed sample combination approach to correctly capture the high proportion of zeros observed in the data or, more specifically, to accurately estimate the joint probability $\operatorname{Pr}\left(X_{F H}=0, X_{M H}=0\right)$. However, when conditioning the analysis on non-zero data, the sample combination outcome and the empirical histograms align quite closely; see Figure 3.4. It is also clear from comparing the upper and middle graphs of these two figures that the outcome of the data combination strategy is identical to that of the bivariate sample joint ECF inversion. This seems to suggest that the discrepancies detected between the estimates and the real data are due more to an imperfect Fourier inversion procedure than to a default in the identification strategy per se.

Tables 3.18 and 3.19 summarize bivariate statistics for various truncation parameter values. The covariance and the Pearson correlation coefficient, as well as three measures of association between discrete variables, are reported. Estimates resulting 
from the sample combination strategy are always reasonably close to bivariate sample statistics for truncation parameter values $T=U=4$. This is consistent with the ISE results reported previously, where $T=U=4$ appeared roughly as optimal truncation values.

To go beyond association measures and assess whether the proposed data combination method can, more generally, accurately capture the dependence between the variables $X_{F H}$ and $X_{M H}$, I also compute distance correlation estimates. Contrary to Pearson correlation coefficient that only measure linear dependence between two random variables, distance correlation measures all types of dependence; see Székely et al. (2007) and Székely and Rizzo (2009). Because it is based on characteristic functions, using this dependence measure is quite natural for the present framework. As can be seen in Table 3.20, this statistic is particularly sensitive to the choice of the truncation parameter. However, reasonable estimates are again obtained with $T=U=4 .^{13}$

Overall, the methodology developed in the preceding chapter seems to perform reasonably well on the type of data dealt with in this application, despite the challenge posed by the highly skewed nature of the distributions that are involved. One way of coping with this difficulty is to work with residuals instead of raw data. An analysis similar to the one just performed on raw data has been carried out on OLS regression residuals. ${ }^{14}$ For the sake of brevity, detailed results are not reported. Figure 3.5 simply illustrates the bivariate density estimates obtained in the case of DC for truncation values as small as $T=U=0.5 \cdot{ }^{15}$ It suggests that the shape of the true bivariate distribution of the residuals is correctly captured by the sample

\footnotetext{
${ }^{13}$ Using this dependence concept, formal testing of the joint independence of $X_{F H}$ and $X_{M H}$ could also be undertaken.

${ }^{14}$ 'Individual' residuals were obtained by regressing independently the individual series $X_{F H}$ and $X_{M H}$ on individual and household characteristics (using MOP data). 'Aggregate' residuals were obtained by regressing the aggregate series $X_{H H}$ on both heads's individual characteristics, as well as household characteristics (using CFM data).

${ }^{15}$ Investigation of empirical ISEs indeed indicate that optimal truncation values are much smaller in the case of residuals than for raw data, close to 1 .
} 
combination approach.

\subsection{Non-Parametric Estimation of Joint Payment Behavior within Couple Households by Sam- ple Combination}

I now proceed to recovering partners' joint distributions by combining MOP individuallevel data and CFM aggregate household data under assumptions of Model 1. Given the conclusion of the compatibility checking exercise, three variables measuring payment behavior are retained: the number of monthly purchases made using CC, SVC and cash.

Figures 3.6 to 3.8 illustrate marginal distributions obtained for various truncation values, and provide for comparison purposes the empirical histograms obtained directly on univariate samples. Further, Table 3.21 summarizes Pearson correlation estimates for different truncation values, as well as estimates obtained directly from univariate sample statistics. These comparisons can serve as simple diagnostic tools to assess the sensitivity of final estimation results to changes in the truncation parameters. For the case of $\mathrm{CC}$, truncation values between 3 and 4 seem to provide most accurate estimates in terms of both marginal distributions and correlation coefficients. By contrast, in the case of cash, higher values around $T=U=5$ seem most appropriate. For the case of SVC however, Figure 3.7 and Table 3.21 provide inconsistent signals. This is most likely due to the fact that the distributions, in the case of SVC, are very highly skewed, hence particularly challenging to estimate.

The final joint PMF estimates are represented on the left-hand side of Figures 3.9 to 3.11. CC and SVC both present high probabilities at $\left(X_{F H}=0, X_{M H}=0\right)$. For cash, the peak in the distribution is spread over several small-valued mass points. In 
each case, I juxtaposed a graph of the product of the estimated marginal distributions of $X_{F H}$ and $X_{M H}$. Two variables are dependent if and only if their joint distribution is different from the product of the marginal distributions. However, formal testing procedures are necessary for assessing joint independence of the male and female quantities.

Using non-parametric kernel estimation methods, Ahmad and Li (1997) propose a consistent test for independence of two random vectors based on the $L_{2}$ norm of difference between the joint density and the product of their marginals; see also Henderson and Parmeter (2015). Since my second-step estimator is a special case of the deconvolution kernel estimator, this kernel-based test for independence should be adaptable for my purpose. Alternatively, as already mentioned, an independence test based on characteristic functions has been proposed by Székely et al. (2007). It relies on a measure of distance between the joint $\mathrm{CF}$ and the product of the marginal CFs called the distance covariance. Indeed, in terms of characteristic functions, $X_{F H}$ and $X_{M H}$ are independent if and only $\phi_{X_{F H}, X_{M H}}=\phi_{X_{F H}} \phi_{X_{M H}}$. Implementation of these formal tests is left for future work.

More straightforwardly, once the joint distribution functions are estimated, several measures of dependence can be computed. One dependence measure that can be derived from the joint PMF estimates is mutual information. This dependence concept from information theory was first introduced by Shannon (1948), and describes how much information two random variables share with each other. Table 3.22 presents mutual information estimates for the estimated joint distribution of male and female monthly purchases paid using CC, SVC and cash. As can be seen in the table, one obvious shortcoming of this dependence measure for our purpose is that it is quite sensitive to the truncation parameter values. Overall, these estimates are relatively small.

Distance correlation estimates are also reported in Table 3.23. For CC and cash 
these statistics seem reasonable inasmuch as their values lie between 0 and 1 . They also appear relatively insensitive to changes in the truncation limits. For SVC however, distance correlation measures derived from joint PMF estimates are largely above the theoretical upper bound of 1 . Note however that when the analysis is conditioned on non-zero values only, more reasonable values around 0.17 are obtained. It is interesting to compare these distance correlation statistics with the sample Pearson coefficient estimates of Table 3.21. In particular, in the case of SVC and cash, the linear correlation coefficients are small and can be shown to be statistically insignificant; cf. Table 3.16. However, the distance correlation statistics are quite larger. Although no formal test is performed here, the distance correlation measures seem to detect a nonlinear dependence between $X_{F H}$ and $X_{M H}$ that Pearson correlation would leave unnoticed.

The estimated joint distribution functions could of course be investigated further. For example, Chi-plots can be used to examine tail dependence in the data, following Fisher and Switzer $(1985,2001)$. The short analysis performed in this section has indeed highlighted the fact that linear measures of dependence are likely to be too limited to describe intra-household dependence with respect to payment behaviors. Also, conditional analysis on residuals could be undertaken.

\subsection{Conclusion}

In this chapter, I apply the methodology developed in Chapter 2 to analyze intrahousehold payment behaviors in the absence of intra-household data. This is achieved by combining individual-level data and household aggregated data. The identification and estimation approaches seem to perform accurately in this real data application, despite some challenging features of the variables of interest. The latter are indeed highly skewed in regards to their distributions, with a large proportion of observations 
situated on one single mass point.

A short analysis of the estimated joint distribution functions was presented in Section 3.6. While potential extensions are numerous, it points to the presence of potentially nonlinear intra-household interactions with respect to couple payment behaviors. If ignored, the latter might lead to spurious results due for instance to omitted variable bias. 


\section{References}

Ahmad, I. A. AND Q. Li (1997): "Testing independence by nonparametric kernel method," Statistics 8 probability letters, 34, 201-210.

AraAr, A. And J.-Y. Duclos (2007): "DASP: Distributive Analysis Stata Package," .

Arango, C., K. P. Huynh, B. Fung, And G. Stuber (2012): "The changing landscape for retail payments in canada and the Implications for the demand for cash," Bank of Canada Review, 2012, 31-40.

Arango, C. And A. Welte (2012): “The Bank of Canada's 2009 Methods-ofPayment Survey: Methodology and Key Results," Staff Discussion Paper 20126, Bank of Canada.

Burdett, K., M. Dong, L. Sun, And R. Wright (2016): "Marriage, Markets, and Money: A Coasian Theory of Household Formation," International Economic Review, 57, 337-368.

Buskirk, T. D. AND S. L. LoHR (2005): "Asymptotic properties of kernel density estimation with complex survey data," Journal of Statistical Planning and Inference, $128,165-190$.

Chen, H., M.-H. Felt, And K. P. Huynh (forthcoming): "Retail payment innovations and cash usage: Accounting for attrition using refreshment samples," Journal of the Royal Statistical Society: Series A.

Fisher, N. AND P. Switzer (1985): "Chi-plots for assessing dependence," Biometrika, 72, 253-265.

- (2001): "Graphical assessment of dependence: Is a picture worth 100 tests?" The American Statistician, 55, 233-239.

Fung, B., K. P. Huynh, And G. Stuber (2015): "The use of Cash in Canada," Bank of Canada Review, 2015, 45-56.

Fung, B. S., K. P. Huynh, And L. SABetTi (2014): "The impact of retail payment innovations on cash usage," Journal of Financial Market Infrastructures, $3,3-31$.

Henderson, D. J. And C. F. Parmeter (2015): Applied nonparametric econometrics, Cambridge University Press.

Henry, C., K. Huynh, And Q. R. Shen (2015): "2013 Methods-of-Payment Survey Results," Staff Discussion Paper 20154., Bank of Canada.

Pahl, J. (1999): Invisible Money: Family Finances in the Electronic Economy, Policy Press, Bristol. 
- (2005): "Individualisation in couple finances: Who pays for the children?" Social Policy and Society, 4, 381-391.

(2008): "Family finances, individualisation, spending patterns and access to credit," Journal of Behavioral and Experimental Economics (formerly The Journal of Socio-Economics), 37, 577-591.

Shannon, C. E. (1948): "A mathematical theory of communication," Bell System Technical Journal, 27, 379-423.

Stango, V. (2000): "Competition and Pricing in the Credit Card Market," Review of Economics and Statistics, 82, 499-508.

SzÉKELY, G. J. AND M. L. Rizzo (2009): "Brownian distance covariance," The annals of applied statistics, 3, 1236-1265.

SzÉKely, G. J., M. L. Rizzo, And N. K. BAKirov (2007): "Measuring and testing dependence by correlation of distances," The Annals of Statistics, 35, 2769-2794.

Vogler, C., M. Brockmann, and R. D. Wiggins (2006): "Intimate relationships and changing patterns of money management at the beginning of the twentyfirst century1," The British Journal of Sociology, 57, 455-482. 


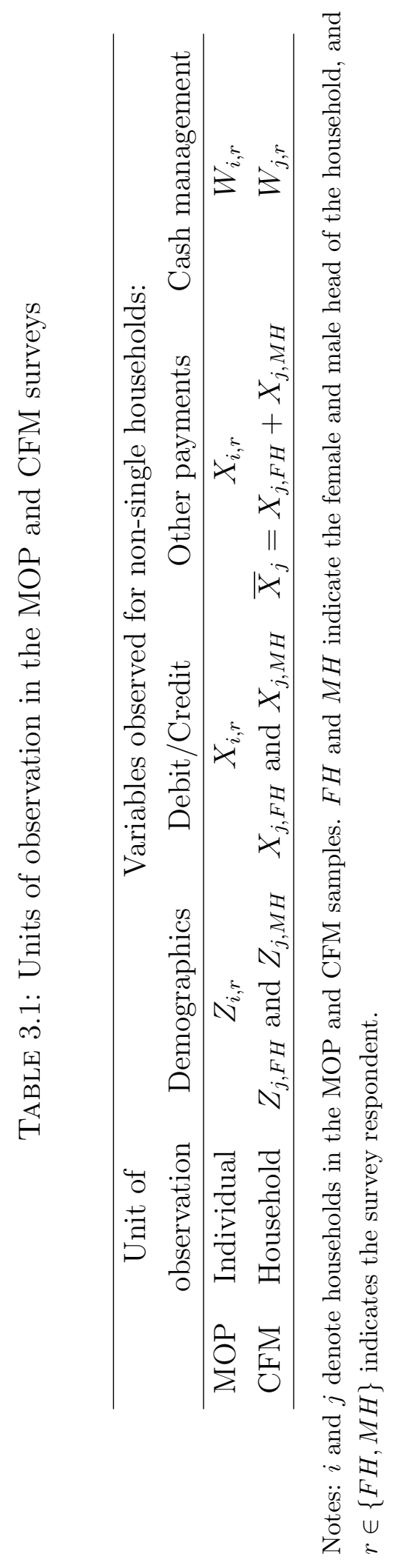


TABle 3.2: Partners' joint probability of usage - CC and DC

\begin{tabular}{|c|c|c|c|}
\hline \multirow{2}{*}{$\mathrm{CC}$} & \multicolumn{2}{|l|}{ MH } & \\
\hline & $\mathrm{N}-\mathrm{U}$ & $\mathrm{U}$ & \\
\hline $\mathrm{FH} \quad \mathrm{N}-\mathrm{U}$ & 40 & 20 & 61 \\
\hline $\mathrm{U}$ & 19 & 20 & 39 \\
\hline & 59 & 41 & $100 \%$ \\
\hline
\end{tabular}

\begin{tabular}{|cc|cc|c}
\hline \multicolumn{2}{|c|}{ DC } & \multicolumn{2}{c|}{ MH } & \\
\multicolumn{2}{c|}{ FH } & N-U & U & \\
& N-U & 73 & 7 & 81 \\
& $\mathrm{U}$ & 10 & 9 & 19 \\
\hline & & 83 & 17 & $100 \%$
\end{tabular}

Notes: Proportions obtained on the CFM subsample of couple households. Number of observations: 5,811 households for CC, 5,838 households for DC. $F H$ and $M H$ stand for female and male head of household; $U$ and $N$ - $U$ indicate usage and non-usage (whether purchases were completed with a method of payment in the past month).

TABLE 3.3: Probability of usage conditional on partner's use - CC and DC

\begin{tabular}{lcc}
\hline CC & FH: & MH: \\
& $\mathrm{U}$ & $\mathrm{U}$ \\
\hline Partner: & & \\
N-U & 32 & 33 \\
$\mathrm{U}$ & 50 & 52 \\
\hline & & \\
\hline DC & FH: & MH: \\
& $\mathrm{U}$ & $\mathrm{U}$ \\
\hline Partner: & & \\
N-U & 12 & 9 \\
$\mathrm{U}$ & 56 & 49 \\
\hline
\end{tabular}

Notes: Proportions obtained on the CFM subsample of couple households. Number of observations: 5,811 households for CC, 5,838 households for DC. Numbers are in proportions. FH and $M H$ stand for female and male head of household; $U$ and $N$ - $U$ indicate usage and non-usage (whether purchases were completed with a method of payment in the past month). 


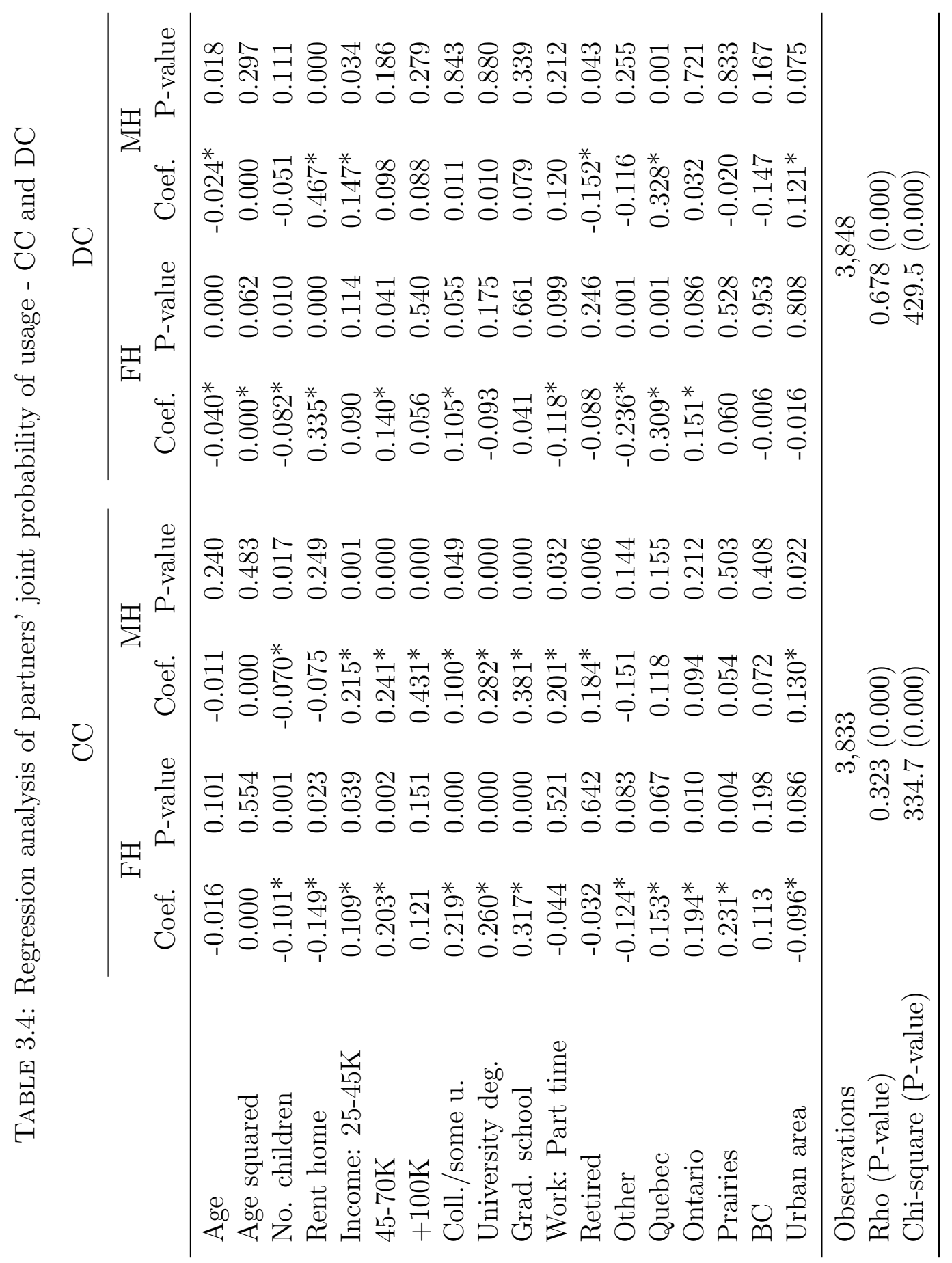

工

它

焉

范

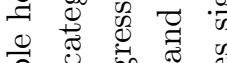

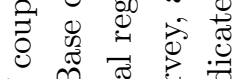

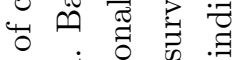

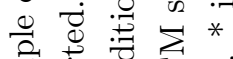

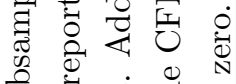

ज

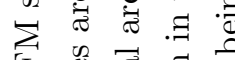

贡

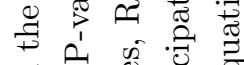

‡.

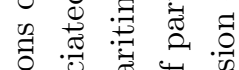

.

की

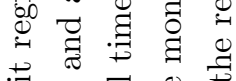

范些寻

䒕.

ن

需 0 论

क

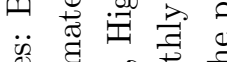

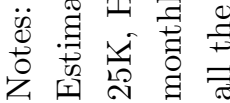


TABLE 3.5: Regression analysis of self and partner methods of payment usage on personal cash management practices - CC and DC

\begin{tabular}{|c|c|c|c|c|c|c|c|c|}
\hline & \multicolumn{4}{|c|}{$\mathrm{CC}$} & \multicolumn{4}{|c|}{$\mathrm{DC}$} \\
\hline & \multicolumn{2}{|c|}{ Cash withdr. } & \multicolumn{2}{|c|}{ Cash on hand } & \multicolumn{2}{|c|}{ Cash withdr. } & \multicolumn{2}{|c|}{ Cash on hand } \\
\hline & Coef. & P-val. & Coef. & P-val. & Coef. & P-val. & Coef. & P-val. \\
\hline Self use & $-0.20^{*}$ & 0.000 & -0.03 & 0.715 & $0.30 *$ & 0.000 & $-0.33^{*}$ & 0.000 \\
\hline Partner use & 0.02 & 0.823 & $0.18^{*}$ & 0.093 & $0.17^{*}$ & 0.043 & $0.32^{*}$ & 0.013 \\
\hline Both use & $-0.17^{*}$ & 0.000 & -0.00 & 0.944 & $0.36^{*}$ & 0.000 & $-0.43^{*}$ & 0.000 \\
\hline \multicolumn{9}{|c|}{ Demographics - Respondent } \\
\hline Female & 0.00 & 0.981 & $-0.16^{*}$ & 0.005 & -0.03 & 0.444 & $-0.11^{*}$ & 0.036 \\
\hline Age & -0.00 & 0.685 & -0.01 & 0.699 & -0.00 & 0.648 & -0.00 & 0.800 \\
\hline Income: $25-45 \mathrm{~K}$ & 0.05 & 0.224 & -0.09 & 0.125 & 0.03 & 0.423 & -0.06 & 0.285 \\
\hline $45-70 \mathrm{~K}$ & $0.19^{*}$ & 0.000 & $0.16^{*}$ & 0.017 & $0.16^{*}$ & 0.000 & $0.17^{*}$ & 0.010 \\
\hline$+100 \mathrm{~K}$ & $0.15^{*}$ & 0.006 & $0.40^{*}$ & 0.000 & $0.13^{*}$ & 0.014 & $0.37^{*}$ & 0.000 \\
\hline Coll./some u. & 0.03 & 0.390 & -0.04 & 0.492 & 0.02 & 0.647 & -0.06 & 0.309 \\
\hline University deg. & -0.06 & 0.204 & 0.00 & 0.999 & -0.06 & 0.204 & -0.07 & 0.303 \\
\hline Grad. school & -0.03 & 0.601 & -0.05 & 0.571 & -0.02 & 0.745 & -0.09 & 0.279 \\
\hline Work: Part time & -0.06 & 0.229 & $0.13^{*}$ & 0.099 & -0.07 & 0.132 & $0.13^{*}$ & 0.086 \\
\hline Retired & -0.07 & 0.181 & 0.10 & 0.199 & -0.07 & 0.133 & 0.10 & 0.170 \\
\hline Other & -0.01 & 0.876 & -0.01 & 0.317 & 0.03 & 0.583 & -0.12 & 0.144 \\
\hline Born out. Can. & $-0.10^{*}$ & 0.017 & 0.00 & 0.978 & $-0.07^{*}$ & 0.066 & -0.01 & 0.855 \\
\hline \multicolumn{9}{|c|}{ Demographics - Partner } \\
\hline Age & $0.03^{*}$ & 0.001 & $0.04^{*}$ & 0.019 & $0.03^{*}$ & 0.001 & $0.03^{*}$ & 0.032 \\
\hline Income: $25-45 \mathrm{~K}$ & 0.02 & 0.616 & $0.10^{*}$ & 0.085 & 0.01 & 0.782 & $0.11^{*}$ & 0.063 \\
\hline $45-70 \mathrm{~K}$ & -0.03 & 0.559 & 0.09 & 0.170 & -0.01 & 0.767 & 0.06 & 0.341 \\
\hline$+100 \mathrm{~K}$ & -0.04 & 0.420 & 0.11 & 0.167 & -0.03 & 0.520 & $0.14^{*}$ & 0.086 \\
\hline Coll./some u. & -0.03 & 0.448 & $-0.19^{*}$ & 0.000 & -0.02 & 0.474 & $-0.15^{*}$ & 0.002 \\
\hline University deg. & $-0.15^{*}$ & 0.001 & 0.07 & 0.287 & $-0.14^{*}$ & 0.002 & 0.10 & 0.153 \\
\hline Grad. school & -0.10 & 0.101 & -0.15 & 0.111 & -0.08 & 0.176 & -0.12 & 0.180 \\
\hline Work: Part time & $-0.09^{*}$ & 0.093 & 0.00 & 0.962 & $-0.10^{*}$ & 0.059 & 0.01 & 0.953 \\
\hline Retired & -0.07 & 0.162 & 0.11 & 0.133 & -0.06 & 0.207 & 0.09 & 0.211 \\
\hline Other & $-0.10^{*}$ & 0.078 & 0.00 & 0.989 & -0.07 & 0.199 & 0.03 & 0.777 \\
\hline \multicolumn{9}{|c|}{ Demographics - Household } \\
\hline No. children & $0.06^{*}$ & 0.002 & 0.00 & 0.996 & $0.06^{*}$ & 0.001 & 0.03 & 0.376 \\
\hline Rent home & $0.19^{*}$ & 0.000 & $-0.18^{*}$ & 0.006 & $0.19^{*}$ & 0.000 & -0.10 & 0.134 \\
\hline Urban area & -0.03 & 0.399 & -0.00 & 0.953 & -0.03 & 0.500 & -0.05 & 0.430 \\
\hline Observations & \multicolumn{2}{|c|}{3,697} & \multicolumn{2}{|c|}{3,539} & \multicolumn{2}{|c|}{3,712} & \multicolumn{2}{|c|}{3,551} \\
\hline Pseudo R-squared & \multicolumn{2}{|c|}{0.016} & \multicolumn{2}{|c|}{0.011} & \multicolumn{2}{|c|}{0.022} & \multicolumn{2}{|c|}{0.014} \\
\hline Chi-square (P-value) & \multicolumn{2}{|c|}{$277.3(0.000)$} & \multicolumn{2}{|c|}{$446.0(0.000)$} & \multicolumn{2}{|c|}{$375.8(0.000)$} & \multicolumn{2}{|c|}{$551.9(0.000)$} \\
\hline
\end{tabular}

Notes: Negative binomial regressions on the CFM subsample of couple households. Estimated coefficients and associated P-values are reported. Base categories for individual demographics: Income below 25K, High school, Full time, Born in Canada; for household demographics: Own home, Rural area. Additional regressors included but not reported: age squared, province of residence, share of various types of expenditure in monthly expenses, month of participation in the CFM survey, constants. Chi-square and its P-value correspond to a Wald test of all the parameters in the regression equation being zero. For all models, a likelihood-ratio test strongly rejects the Poisson hypothesis of equidispersion. ${ }^{*}$ indicates significance at the 10 percent level. 
TABLE 3.6: Kolmogorov-Smirnov equality of distribution tests - single and couple households

\begin{tabular}{lcccccc} 
& \multicolumn{2}{c}{ All genders } & \multicolumn{2}{c}{ FH } & \multicolumn{2}{c}{ MH } \\
Tests & D-stat. & P-val. & D-stat. & P-val. & D-stat. & P-val. \\
\hline$C C$ - Volume & & & & & & \\
Test: Single smaller & 0.111 & 0.000 & 0.097 & 0.001 & 0.151 & 0.000 \\
Test: Couple smaller & 0.000 & 1.000 & 0.000 & 1.000 & 0.000 & 1.000 \\
Combined K-S test: & 0.111 & 0.000 & 0.097 & 0.003 & 0.151 & 0.000 \\
\hline DC - Volume & & & & & & \\
Test: Single smaller & 0.056 & 0.012 & 0.057 & 0.100 & 0.064 & 0.066 \\
Test: Couple smaller & 0.000 & 1.000 & 0.000 & 1.000 & -0.005 & 0.982 \\
Combined K-S test: & 0.056 & 0.025 & 0.057 & 0.200 & 0.064 & 0.132 \\
\hline SVC - Volume & & & & & & \\
Test: Single smaller & 0.022 & 0.549 & 0.009 & 0.956 & 0.022 & 0.771 \\
Test: Couple smaller & -0.010 & 0.881 & -0.010 & 0.942 & -0.015 & 0.875 \\
Combined K-S test: & 0.022 & 0.926 & 0.010 & 1.000 & 0.022 & 1.000 \\
\hline$C T C$ - Volume & & & & & & \\
Test: Single smaller & 0.026 & 0.396 & 0.031 & 0.499 & 0.047 & 0.244 \\
Test: Couple smaller & -0.001 & 0.999 & -0.001 & 1.000 & -0.004 & 0.992 \\
Combined K-S test: & 0.026 & 0.744 & 0.031 & 0.878 & 0.047 & 0.481 \\
\hline Cash - Volume & & & & & & \\
Test: Single smaller & 0.001 & 0.998 & 0.004 & 0.988 & 0.000 & 1.000 \\
Test: Couple smaller & -0.100 & 0.000 & -0.042 & 0.277 & -0.157 & 0.000 \\
Combined K-S test: & 0.100 & 0.000 & 0.042 & 0.543 & 0.157 & 0.000 \\
\hline
\end{tabular}

Notes: Results obtained on the 2013 MOP sample. $F H$ and $M H$ stand for female and male head of household. 
TABLE 3.7: Kolmogorov-Smirnov equality of distribution tests - male and female heads of households

\begin{tabular}{lcc} 
Tests & D-statistic & P-value \\
\hline$C C$ - Volume & & \\
Test: MH smaller & 0.002 & 0.998 \\
Test: FH smaller & -0.107 & 0.000 \\
Combined K-S test: & 0.107 & 0.000 \\
\hline DC - Volume & & \\
Test: MH smaller & 0.067 & 0.035 \\
Test: FH smaller & 0.000 & 1.000 \\
Combined K-S test: & 0.067 & 0.070 \\
\hline SVC - Volume & & \\
Test: MH smaller & 0.050 & 0.211 \\
Test: FH smaller & -0.008 & 0.961 \\
Combined K-S test: & 0.050 & 0.418 \\
\hline$C T C$ - Volume & & \\
Test: MH smaller & 0.000 & 1.000 \\
Test: FH smaller & -0.073 & 0.020 \\
Combined K-S test: & 0.073 & 0.040 \\
\hline Cash - Volume & & \\
Test: MH smaller & 0.037 & 0.368 \\
Test: FH smaller & -0.029 & 0.526 \\
Combined K-S test: & 0.037 & 0.700 \\
\hline
\end{tabular}

Notes: Results obtained on the 2013 MOP subsample of couple households. $F H$ and $M H$ stand for female and male head of household. 


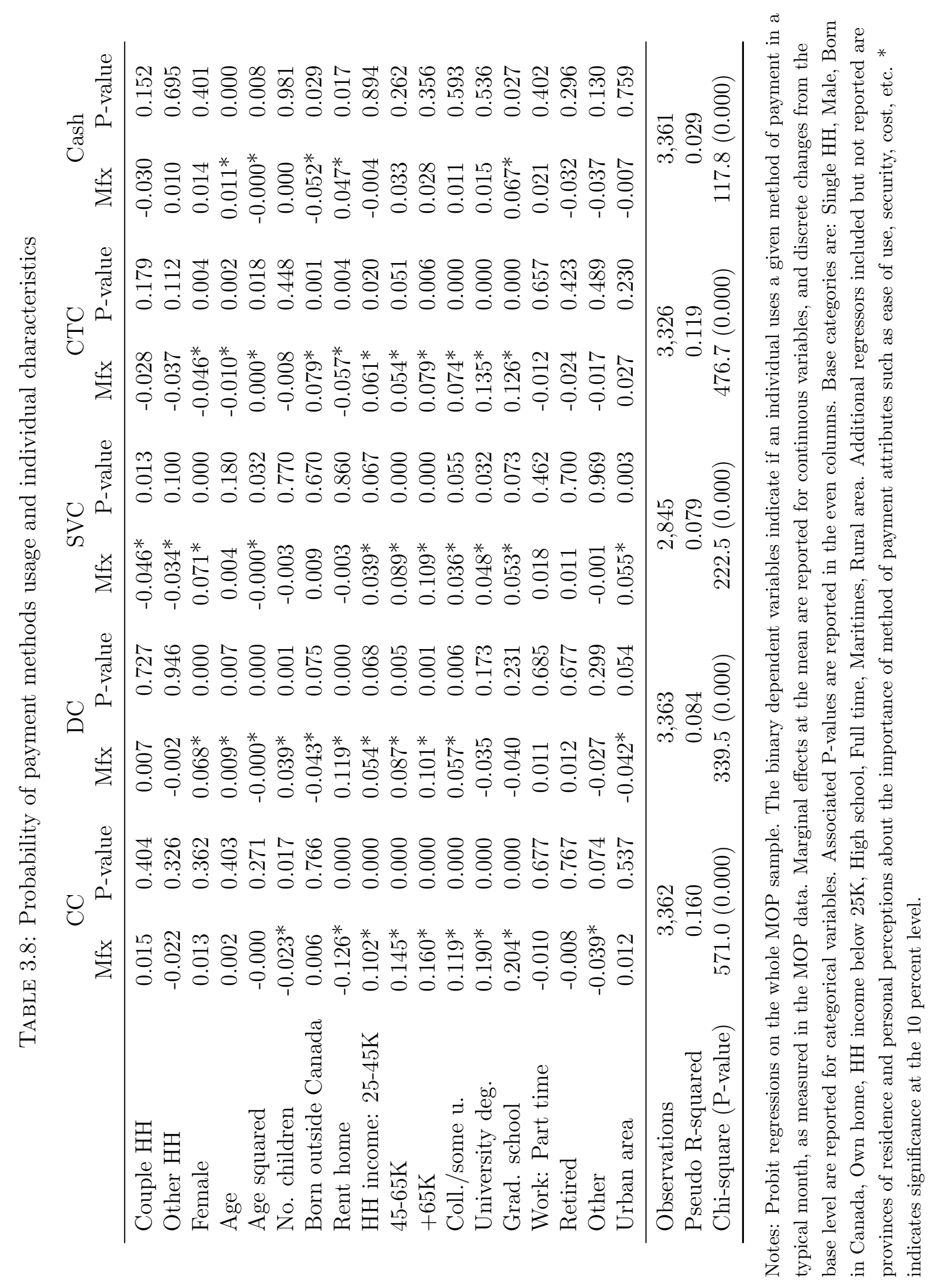




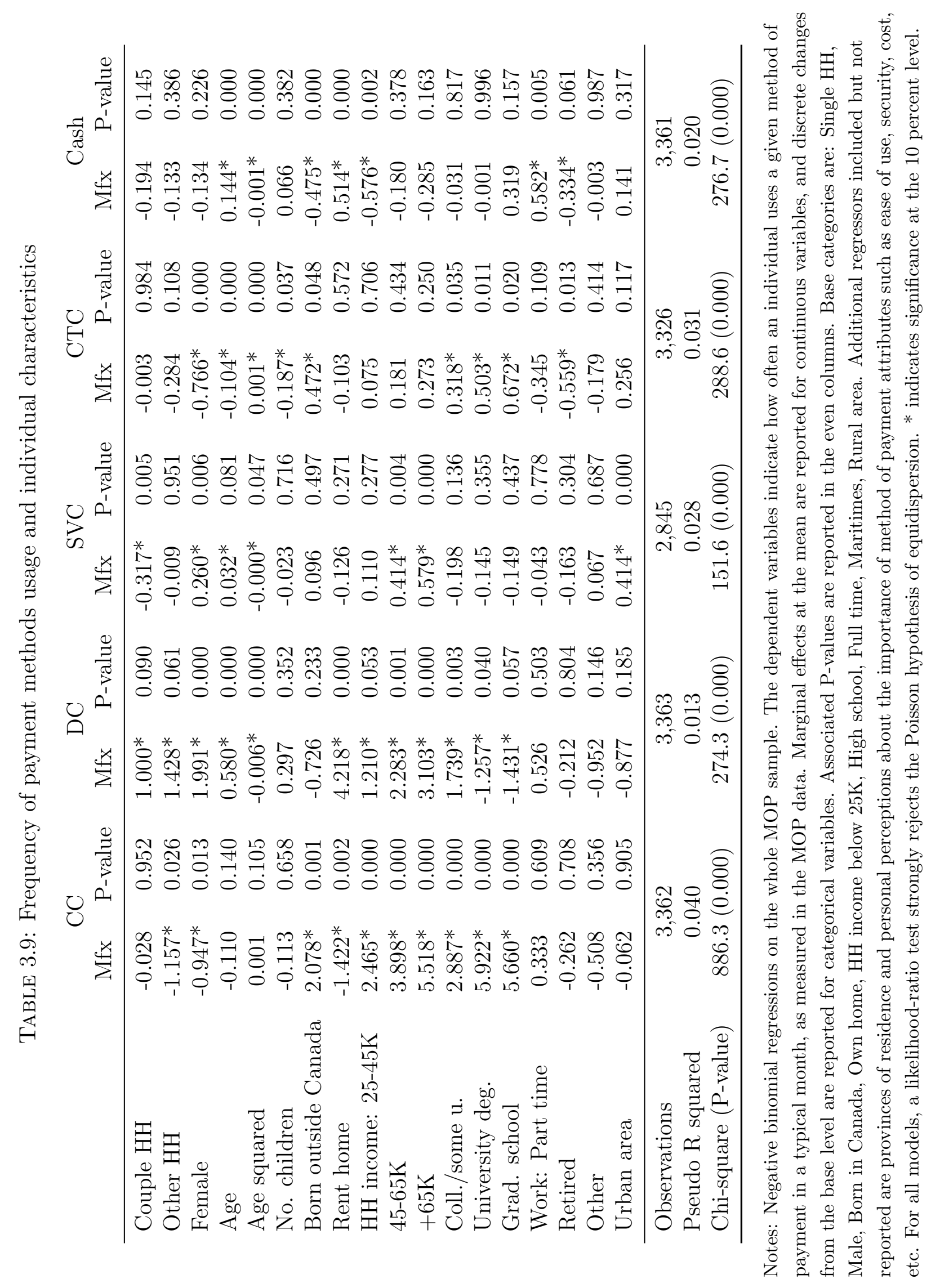




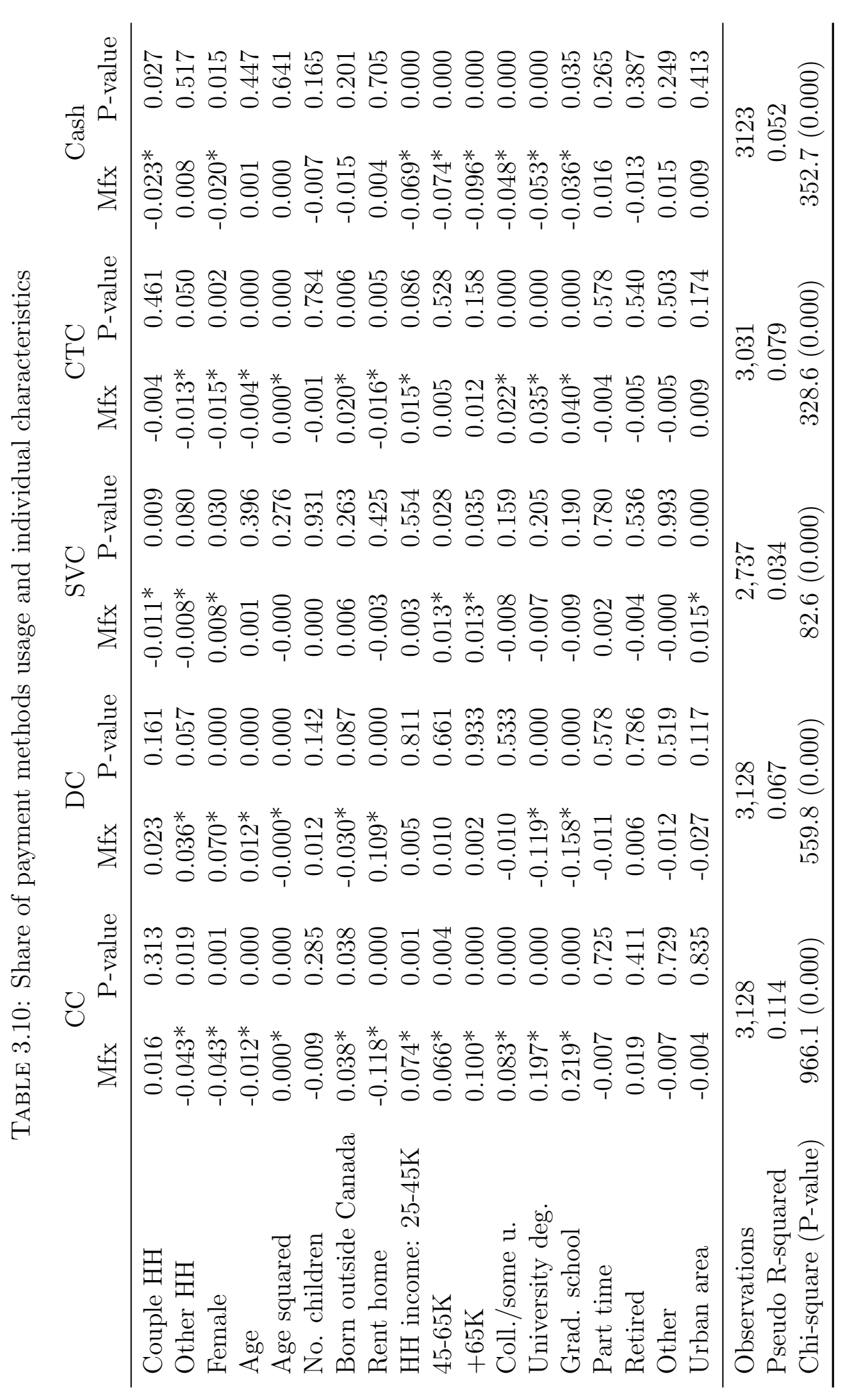

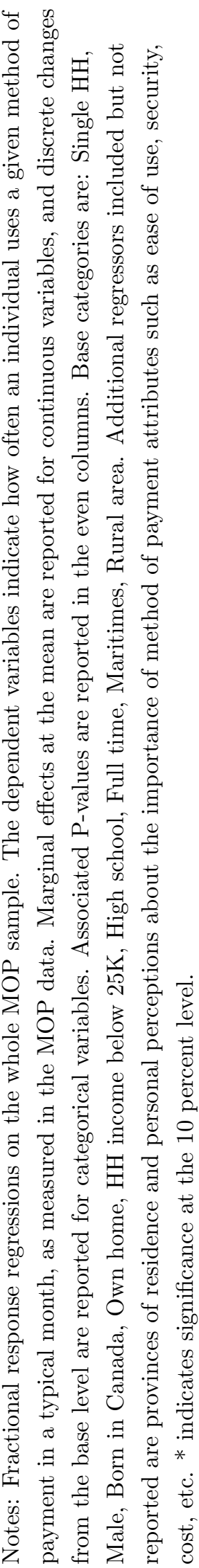




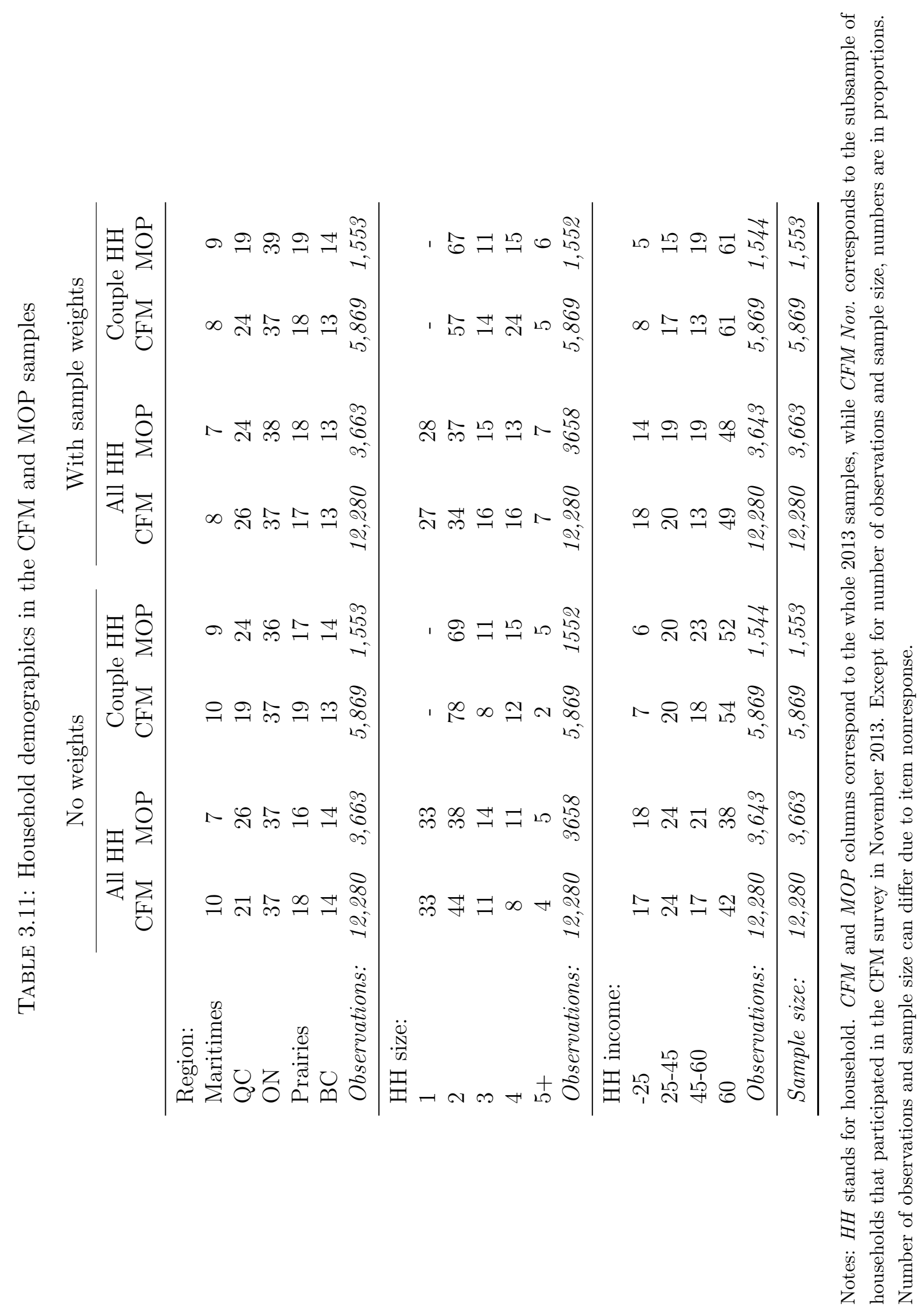




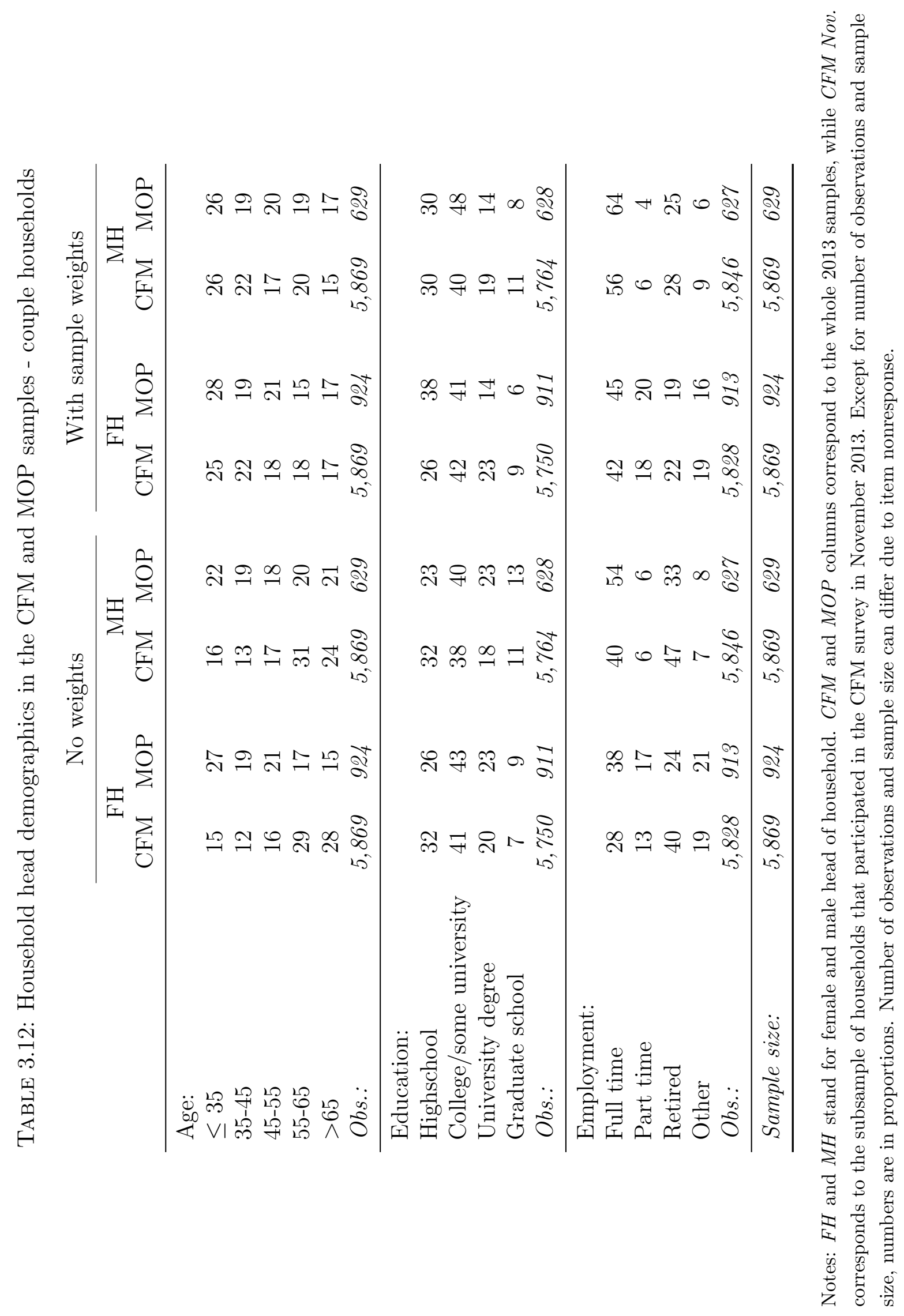


TABLE 3.13: Summary statistics - individual and aggregate variables

\begin{tabular}{lccccccccc} 
Variable & Source & Obs. & Mean & Std. Dev. & Var. & Min. & Max. & Cov. & Corr. \\
\hline CC - Volume & & & & & & & & \\
FH & MOP & 917 & 10.2 & 12.30 & 151.3 & 0 & 50 & 49.7 & 0.29 \\
MH & MOP & 625 & 12.5 & 13.80 & 190.6 & 0 & 60 & & \\
HH total & CFM & 5,817 & 19.1 & 21.01 & 441.3 & 0 & 106 & & \\
\hline DC - Volume & & & & & & & & \\
FH & MOP & 918 & 9.3 & 11.86 & 140.7 & 0 & 50 & 1.2 & 0.01 \\
MH & MOP & 625 & 7.8 & 10.55 & 111.3 & 0 & 45 & & \\
HH total & CFM & 5,833 & 11.2 & 15.95 & 254.4 & 0 & 95 & & \\
\hline SVC - Volume & & & & & & & & \\
FH & MOP & 756 & 0.7 & 1.96 & 3.9 & 0 & 10 & 0.3 & 0.09 \\
MH & MOP & 521 & 0.6 & 1.66 & 2.8 & 0 & 8 & & \\
HH total & CFM & 5,618 & 1.1 & 2.69 & 7.2 & 0 & 20 & & \\
\hline CTC - Volume & & & & & & & & \\
FH & MOP & 903 & 1.4 & 3.30 & 10.9 & 0 & 15 & -9.3 & -0.61 \\
MH & MOP & 617 & 2.2 & 4.62 & 21.3 & 0 & 20 & & \\
HH total & CFM & 5,634 & 1.3 & 3.68 & 13.6 & 0 & 29 & & \\
\hline Cash - Volume & & & & & & & & \\
FH & MOP & 918 & 1.9 & 2.36 & 5.6 & 0 & 10 & 0.2 & 0.04 \\
MH & MOP & 625 & 2.0 & 2.68 & 7.2 & 0 & 12 & & \\
HH total & CFM & 5,364 & 3.7 & 3.64 & 13.2 & 0 & 20 & & \\
\hline
\end{tabular}

Notes: Summary statistics obtained on the 2013 MOP and 2013 CFM subsamples of couple households. Variables measure the number of purchases made using a method of payment in a month. Individual variables from the MOP have been winsorized at the 97.5th percentile; aggregate variables from the CFM have been winsorized at the 99.5th percentile. $H H$ stands for household; $\mathrm{FH}$ and $\mathrm{MH}$ stand for female and male head of household. The covariance and correlation coefficients reported are between $\mathrm{FH}$ and $\mathrm{MH}$, and obtained by the formula: $\operatorname{Cov}\left(X_{F H}, X_{M H}\right)=1 / 2\left(\operatorname{Var}\left(X_{H H}\right)-\operatorname{Var}\left(X_{F H}\right)-\operatorname{Var}\left(X_{M H}\right)\right)$. 
TABLE 3.14: Confidence intervals for sample means

\begin{tabular}{lccccc} 
& $\mathrm{E}\left(X_{F H}\right)$ & $\mathrm{E}\left(X_{M H}\right)$ & $\mathrm{E}\left(X_{F H}+X_{M H}\right)$ & \multicolumn{2}{c}{$\mathrm{E}\left(X_{H H}\right)$} \\
\hline Source & MOP & MOP & MOP & CFM & CFM Nov. \\
\hline CC - Volume & & & & & \\
Estimate & 10.20 & 12.49 & 22.69 & 19.09 & 21.17 \\
Std. error & 0.41 & 0.55 & 0.69 & 0.28 & 1.09 \\
LB, 95\% CI & 9.40 & 11.41 & 21.35 & 18.55 & 19.04 \\
UP, 95\% CI & 11.00 & 13.58 & 24.04 & 19.63 & 23.31 \\
\hline DC - Volume & & & & & \\
Estimate & 9.35 & 7.78 & 17.12 & 11.22 & 10.50 \\
Std. error & 0.39 & 0.42 & 0.58 & 0.21 & 0.63 \\
LB, 95\% CI & 8.58 & 6.95 & 16.00 & 10.81 & 9.26 \\
UP, 95\% CI & 10.12 & 8.60 & 18.25 & 11.63 & 11.74 \\
\hline SVC - Volume & & & & & \\
Estimate & 0.72 & 0.58 & 1.30 & 1.08 & 1.12 \\
Std. error & 0.07 & 0.07 & 0.10 & 0.04 & 0.12 \\
LB, 95\% CI & 0.58 & 0.43 & 1.10 & 1.01 & 0.88 \\
UP, 95\% CI & 0.86 & 0.72 & 1.50 & 1.15 & 1.36 \\
\hline CTC - Volume & & & & & \\
Estimate & 1.45 & 2.24 & 3.69 & 1.28 & 1.57 \\
Std. error & 0.11 & 0.19 & 0.22 & 0.05 & 0.18 \\
LB, 95\% CI & 1.23 & 1.87 & 3.26 & 1.19 & 1.22 \\
UP, 95\% CI & 1.67 & 2.60 & 4.11 & 1.38 & 1.91 \\
\hline Cash - Volume & & & & & \\
Estimate & 1.92 & 2.04 & 3.95 & 3.71 & 3.51 \\
Std. error & 0.08 & 0.11 & 0.13 & 0.05 & 0.15 \\
LB, 95\% CI & 1.77 & 1.83 & 3.70 & 3.61 & 3.21 \\
UP, 95\% CI & 2.07 & 2.25 & 4.21 & 3.80 & 3.81 \\
\hline
\end{tabular}

Notes: $95 \%$ confidence intervals for the means in the following subsamples: female heads in MOP couple households, male heads in MOP couple households, CFM couple households, CFM couple households that participated in the CFM survey in November. In the third column, $95 \%$ confidence intervals for the sum of the means in the two MOP subsamples are indicated. $H H$ stands for household; $F H$ and $M H$ stand for female and male head of household. 
TABLE 3.15: Confidence intervals for sample proportions

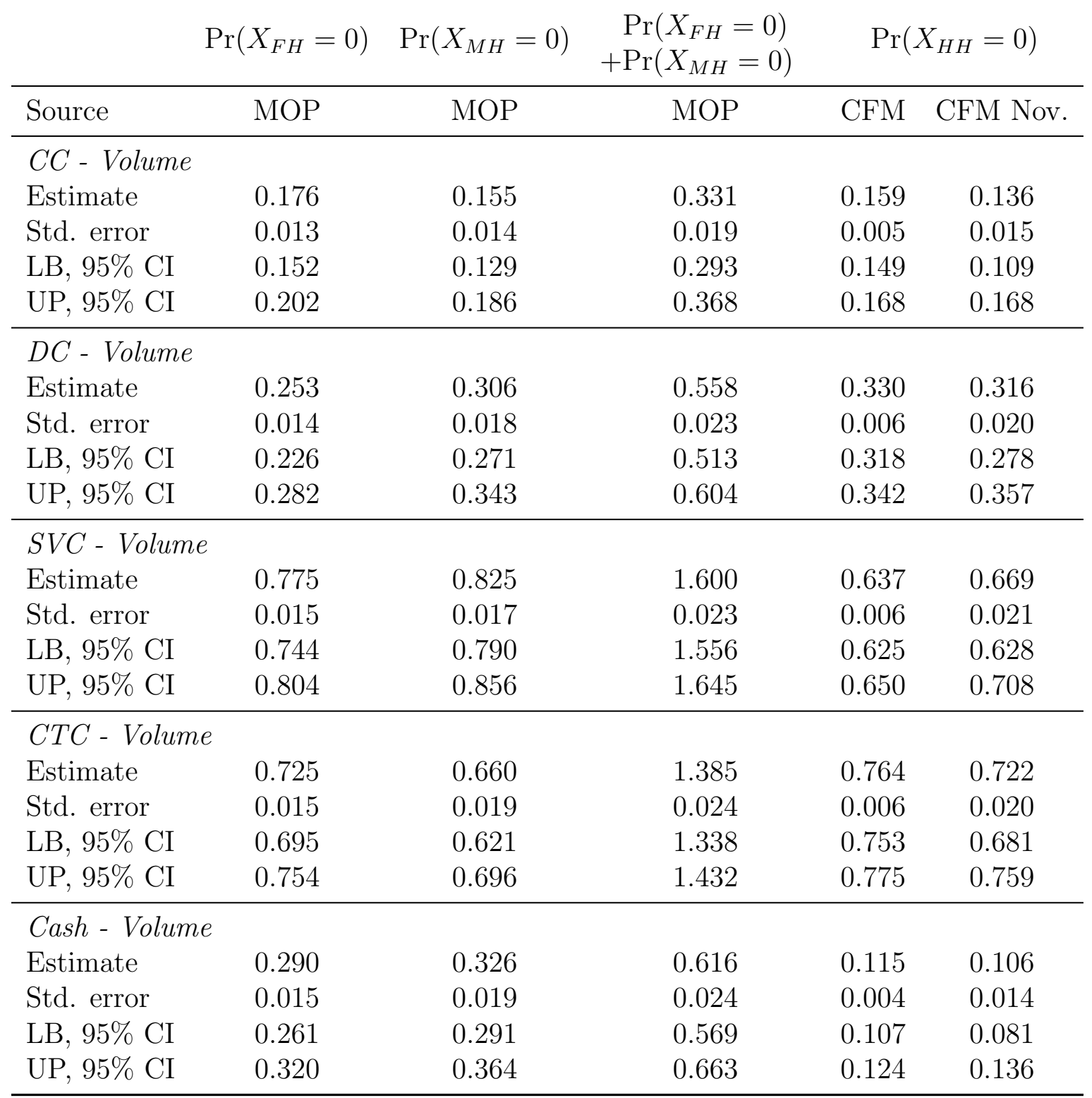

Notes: $95 \%$ confidence intervals for the proportion of zeros in the following subsamples: female heads in MOP couple households, male heads in MOP couple households, CFM couple households, CFM couple households that participated in the CFM survey in November. In the third column, $95 \%$ confidence intervals for the sum of the proportion of zeros in the two MOP subsamples are indicated. $H H$ stands for household; $F H$ and $M H$ stand for female and male head of household. 


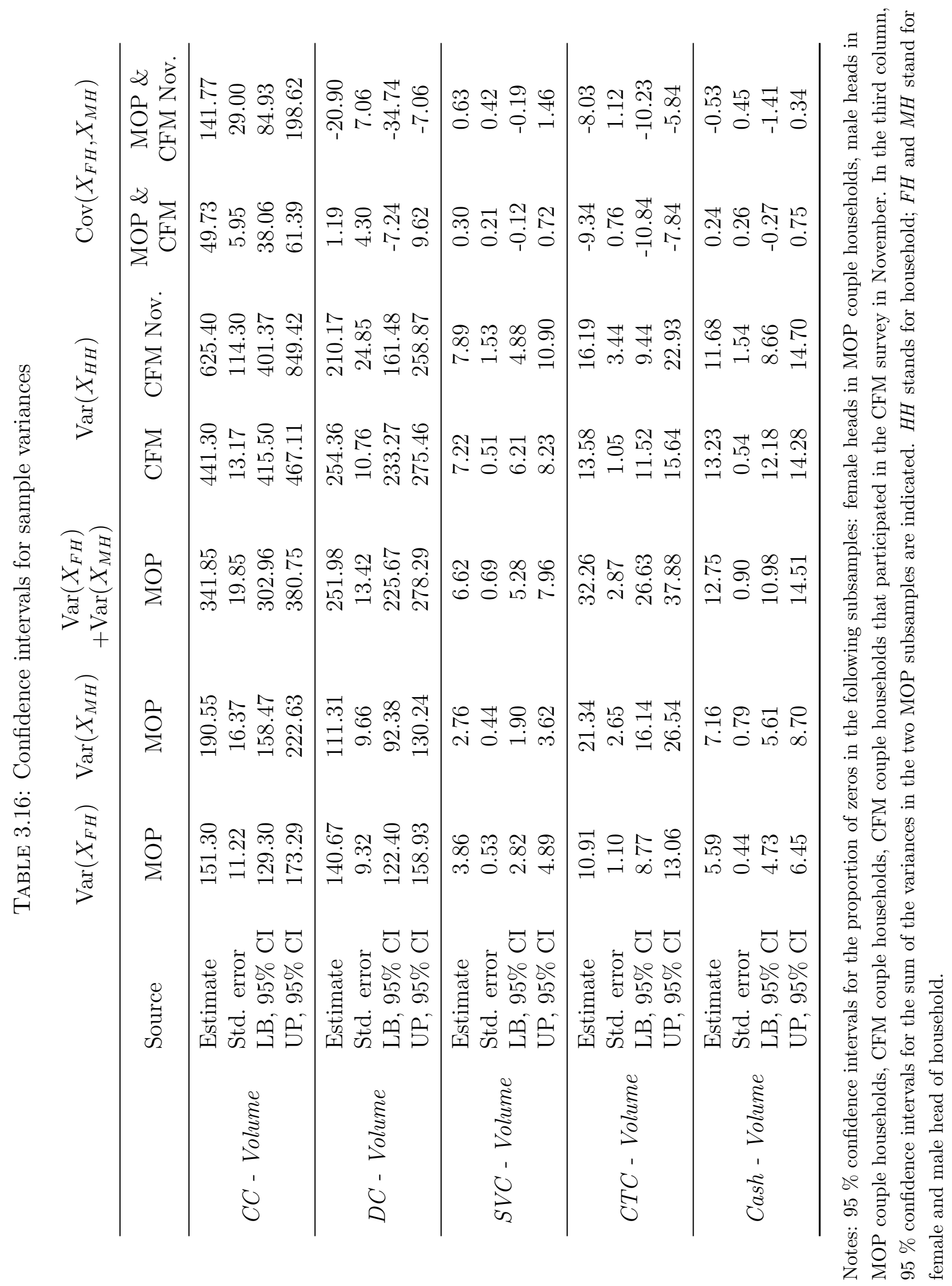


TABLE 3.17: Summary statistics - test data

Variable Obs. Mean Std. Dev. Var. Min. Max. Cov. Corr.

\begin{tabular}{lcccccccc}
\hline \multicolumn{2}{l}{$C C$ - Volume } & & & & & & & \\
$\mathrm{FH}$ & 5,835 & 5.2 & 23.40 & 547.8 & 0 & 931 & 84.8 & 0.19 \\
$\mathrm{MH}$ & 5,832 & 6.2 & 19.00 & 360.8 & 0 & 820 & & \\
$\mathrm{FH}+\mathrm{MH}$ & 5,811 & 11.4 & 32.89 & 1081.5 & 0 & 1320 & & \\
\hline \multicolumn{2}{l}{$\mathrm{DC}-$ Volume } \\
$\mathrm{FH}$ & 5,848 & 2.3 & 11.51 & 132.4 & 0 & 503 & 20.0 & 0.25 \\
$\mathrm{MH}$ & 5,853 & 1.9 & 6.92 & 47.9 & 0 & 213 & & \\
$\mathrm{FH}+\mathrm{MH}$ & 5,838 & 4.2 & 14.85 & 220.6 & 0 & 503 & & \\
\hline
\end{tabular}

Notes: Summary statistics obtained on the 2013 CFM subsamples of couple households. Variables measure the number of purchases made using a method of payment in a month. $H H$ stands for household; $F H$ and $M H$ stand for female and male head of household. The line 'FH+MH' corresponds to the variable $X_{F H+M H}=X_{F H}+X_{M H}$, obtained by summing individual quantities of the female and male heads of each household.

TABLE 3.18: Covariance and Pearson's correlation estimated on test data - CC and $\mathrm{DC}$ in volume, raw data

\begin{tabular}{|c|c|c|c|c|c|c|c|}
\hline & \multicolumn{3}{|c|}{ Covariance } & \multicolumn{3}{|c|}{ Correlation } \\
\hline & & $T=2$ & $T=3$ & $T=4$ & $T=2$ & $T=3$ & $T=4$ \\
\hline \multirow{3}{*}{$\mathrm{CC}$} & Bivariate sample statistic & & 84.8 & & & 0.190 & \\
\hline & Using sample combination & 144.6 & 116.4 & 87.7 & 0.324 & 0.261 & 0.197 \\
\hline & Using joint ECF & 144.6 & 116.5 & 87.8 & 0.324 & 0.261 & 0.197 \\
\hline \multirow{3}{*}{ DC } & Bivariate sample statistic & & 20.0 & & & 0.251 & \\
\hline & Using sample combination & 40.7 & 32.3 & 19.4 & 0.510 & 0.405 & 0.243 \\
\hline & Using joint ECF & 40.7 & 32.3 & 19.4 & 0.510 & 0.405 & 0.243 \\
\hline
\end{tabular}

Notes: PMF estimations are obtained on the support $\{0,1, \ldots, M-1\}$, with $M=2^{5}$ for CC and $M=2^{4}$ for DC. These values correspond approximately to the 95 th percentile of the marginal distributions. $T=U$. 


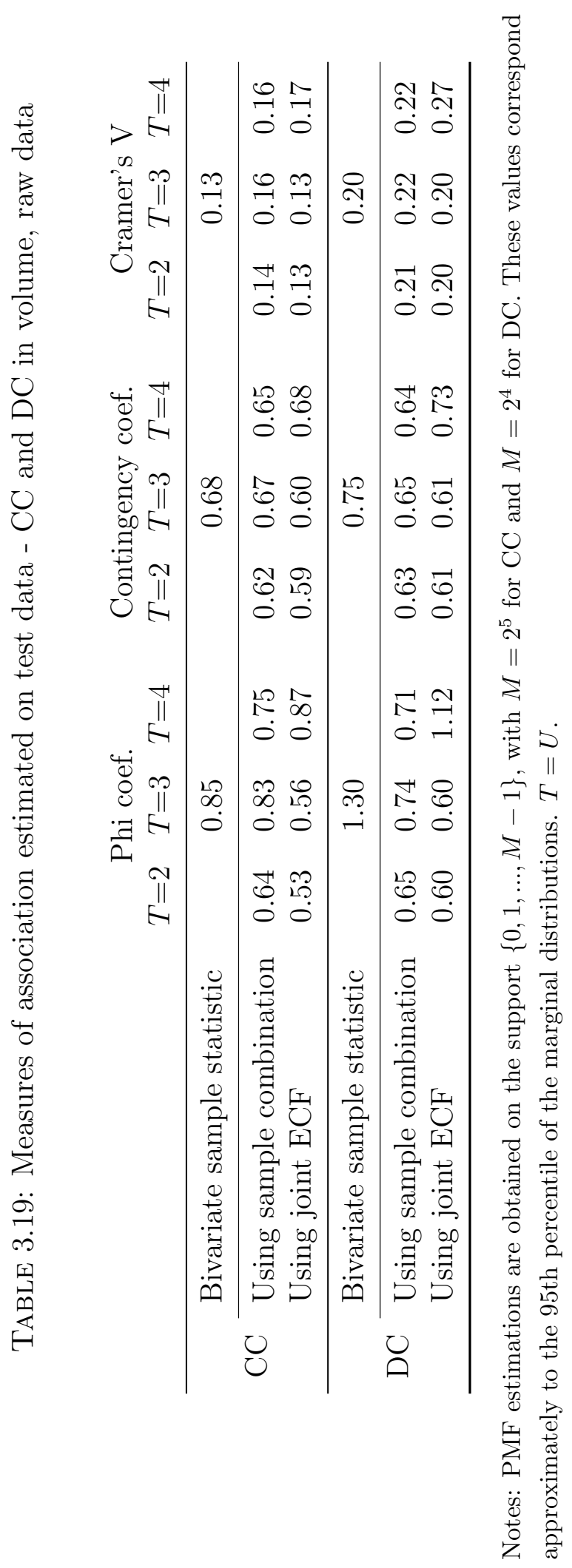


TABle 3.20: Distance correlation estimated on test data - CC and DC in volume, raw data

\begin{tabular}{lllll} 
& & \multicolumn{3}{c}{ Distance correlation } \\
& & $T=2$ & $T=3$ & $T=4$ \\
\hline \multirow{2}{*}{ CC } & Bivariate sample statistic & & 0.160 & \\
& Using sample combination & 0.012 & 0.051 & 0.168 \\
\hline \multirow{2}{*}{ DC } & Bivariate sample statistic & & 0.419 & \\
& Using sample combination & 0.058 & 0.247 & 0.696 \\
\hline
\end{tabular}

Notes: PMF estimations are obtained on the support $\{0,1, \ldots, M-1\}$, with $M=2^{5}$ for $\mathrm{CC}$ and $M=2^{4}$ for DC. These values correspond approximately to the 95 th percentile of the marginal distributions. $T=U$. 
TABLE 3.21: Pearson's correlation estimates

\begin{tabular}{lccccc} 
& \multirow{2}{*}{ Sample statistics } & \multicolumn{4}{c}{ Using sample combination } \\
\cline { 3 - 6 } & & $T=2$ & $T=3$ & $T=4$ & $T=5$ \\
\hline CC & 0.293 & 0.181 & 0.357 & 0.355 & 1.000 \\
SVC & 0.092 & 0.096 & 0.145 & 0.196 & 0.918 \\
Cash & 0.038 & -0.003 & 0.009 & -0.006 & 0.052 \\
\hline
\end{tabular}

Notes: Sample correlation statistics are obtained by combining sample variances of the three marginal distributions, as in Table 3.13. Other estimates are obtained using joint PMF estimated by sample combination, with different truncation values. PMF estimations are obtained on the whole supports of the individual variables $X_{F H}$ and $X_{M H} . T=U$.

TABLE 3.22: Average mutual information estimates

\begin{tabular}{lcccc} 
& $T=2$ & $T=3$ & $T=4$ & $T=5$ \\
\hline CC & 0.293 & 0.203 & 0.042 & $-7.87 \mathrm{E}+17$ \\
SVC & 0.069 & 0.073 & -0.053 & -1.409 \\
Cash & 0.059 & 0.067 & 0.101 & 0.196 \\
\hline
\end{tabular}

Notes: Mutual information statistics are computed using joint PMF estimated by sample combination, for different truncation values. PMF estimations are obtained on the whole supports of the individual variables $X_{F H}$ and $X_{M H} . T=U$.

TABLE 3.23: Distance correlation estimates

\begin{tabular}{lccc} 
& $T=2$ & $T=3$ & $T=4$ \\
\hline CC & - & 0.171 & 0.191 \\
SVC & 6.311 & 3.934 & 3.469 \\
Cash & 0.202 & 0.197 & 0.162 \\
\hline
\end{tabular}

Notes: Distance correlation statistics are computed using joint PMF estimated by sample combination, for different truncation values. PMF estimations are obtained on the whole supports of the individual variables $X_{F H}$ and $X_{M H} . T=U$. 
Figure 3.1: Empirical CDFs - male and female heads of households

(a) $\mathrm{CC}$

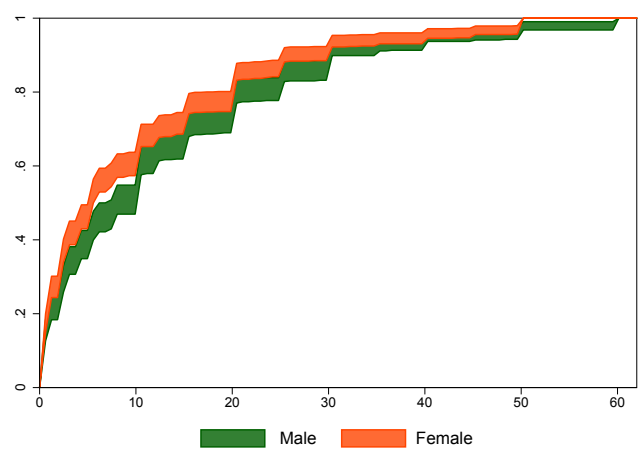

(c) SVC

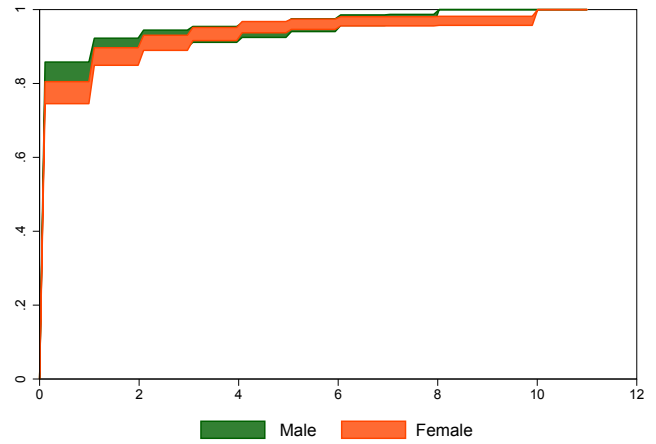

(b) DC

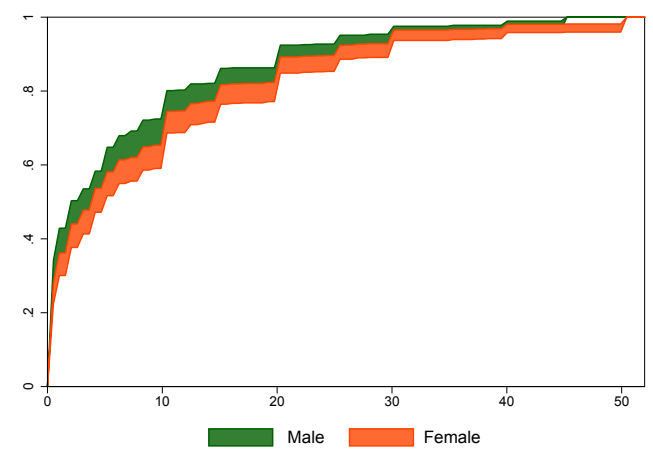

(d) CTC

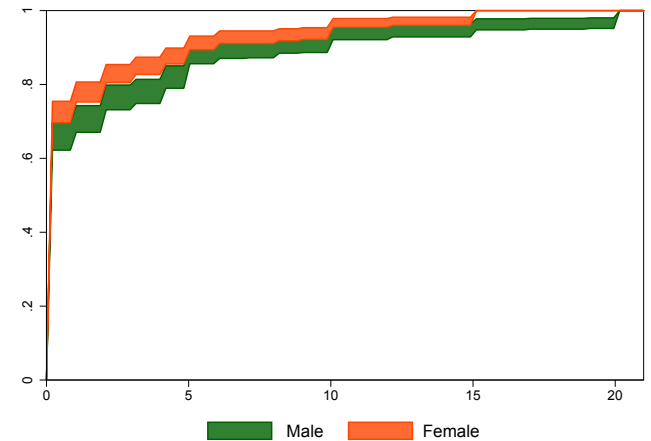

(e) Cash

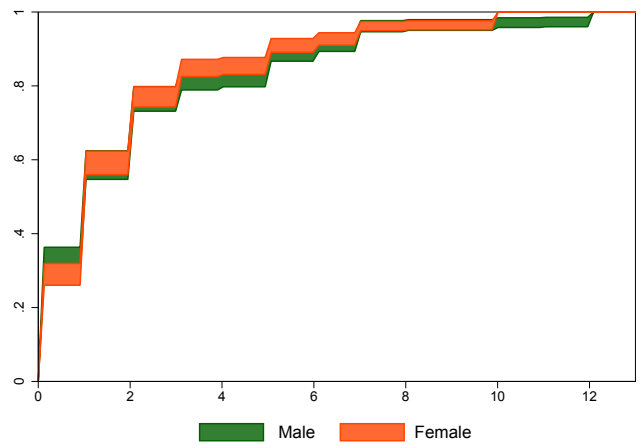

Notes: $95 \%$ confidence intervals of empirical cumulative distribution functions. Stochastic dominance curves of order 1 are drawn using the cfgtsm command of the DASP package; see Araar and Duclos (2007). 


\section{Figure 3.2: Estimated ISE obtained on test data}

(a) CC - Volume

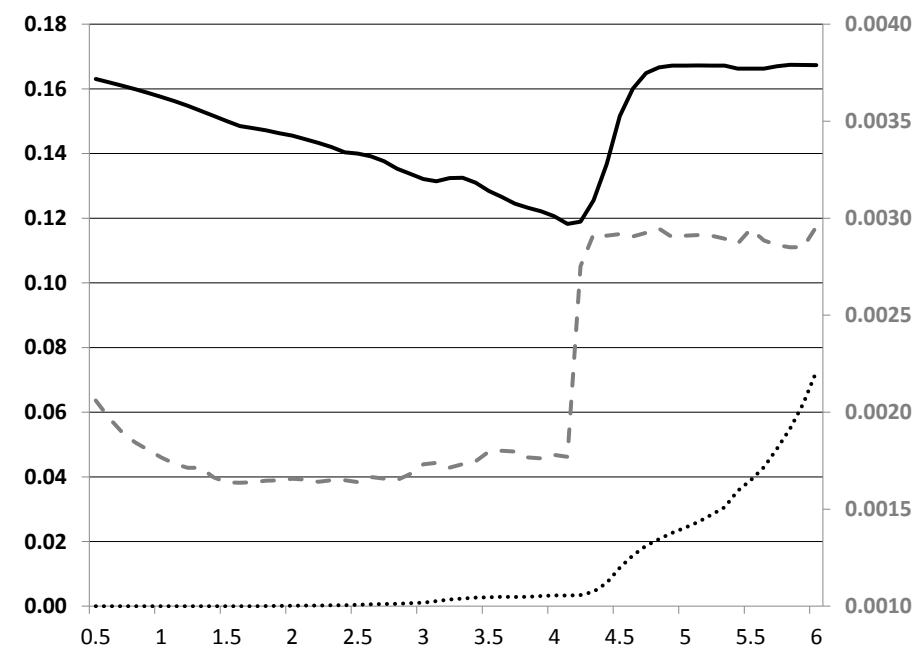

(b) DC - Volume

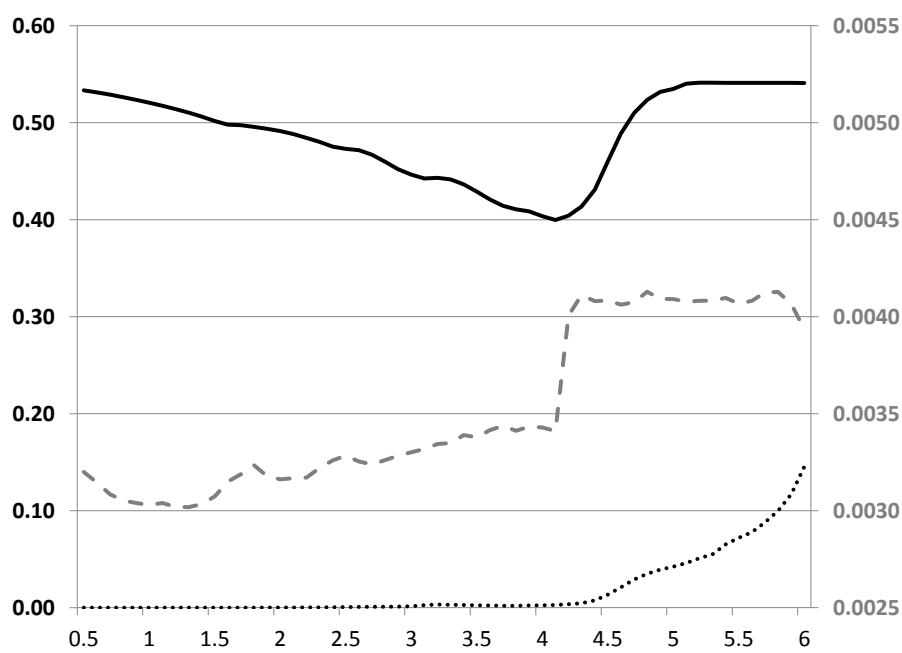

Notes: $T=U$ is in the abscissa. The black solid line, to be read on the left-hand axis, is obtained by comparing the data combination approach to the direct non-parametric PMF estimation on raw data. The grey dashed line, to be read on the right-hand axis, is obtained by comparing the data combination approach to the direct non-parametric PMF estimation on positive values only. The black dotted line, to be read on the left-hand axis, is obtained by comparing the data combination approach to the joint ECF inversion on raw data. 
Figure 3.3: Estimated marginal PMFs obtained on test data - DC in volume, raw data
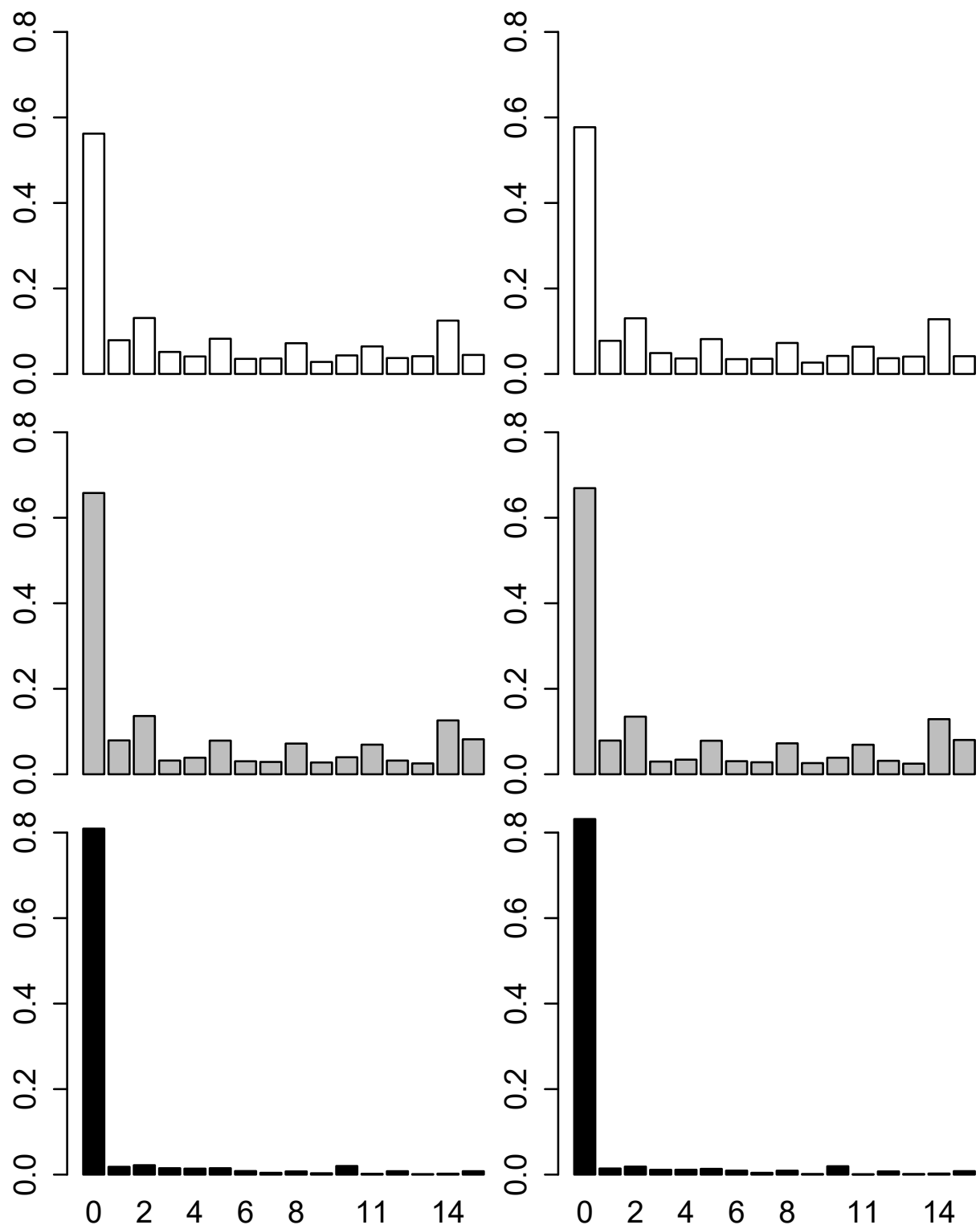

Notes: Marginal PMF estimates for the female head on the left, for the male head on the right. In white, PMF estimates based on sample combination. In grey, PMF estimates based on inversion of the joint ECF of $X_{F H}, X_{M H}$ estimated on the bivariate sample. In black, empirical histograms obtained directly on the univariate samples. $U=T=4$ was used. 
Figure 3.4: Estimated marginal PMFs obtained on test data - DC in volume, non-zero data
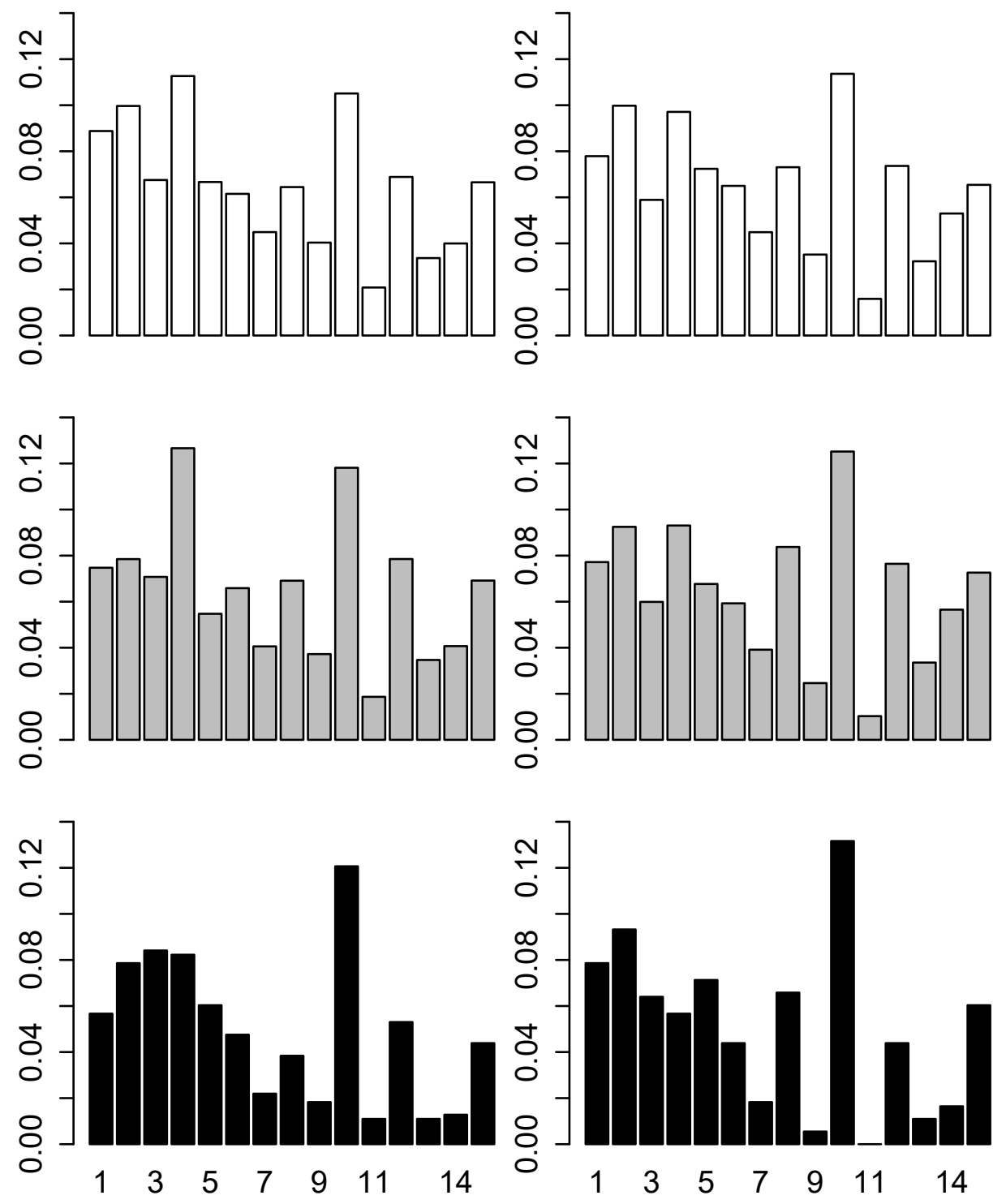

Notes: Marginal PMF estimates for the female head on the left, for the male head on the right. In white, PMF estimates based on sample combination. In grey, PMF estimates based on inversion of the joint ECF of $X_{F H}, X_{M H}$ estimated on the bivariate sample. In black, empirical histograms obtained directly on the univariate samples. $U=T=4$ was used. 
Figure 3.5: Estimated joint density obtained on test data - DC in volume, residuals

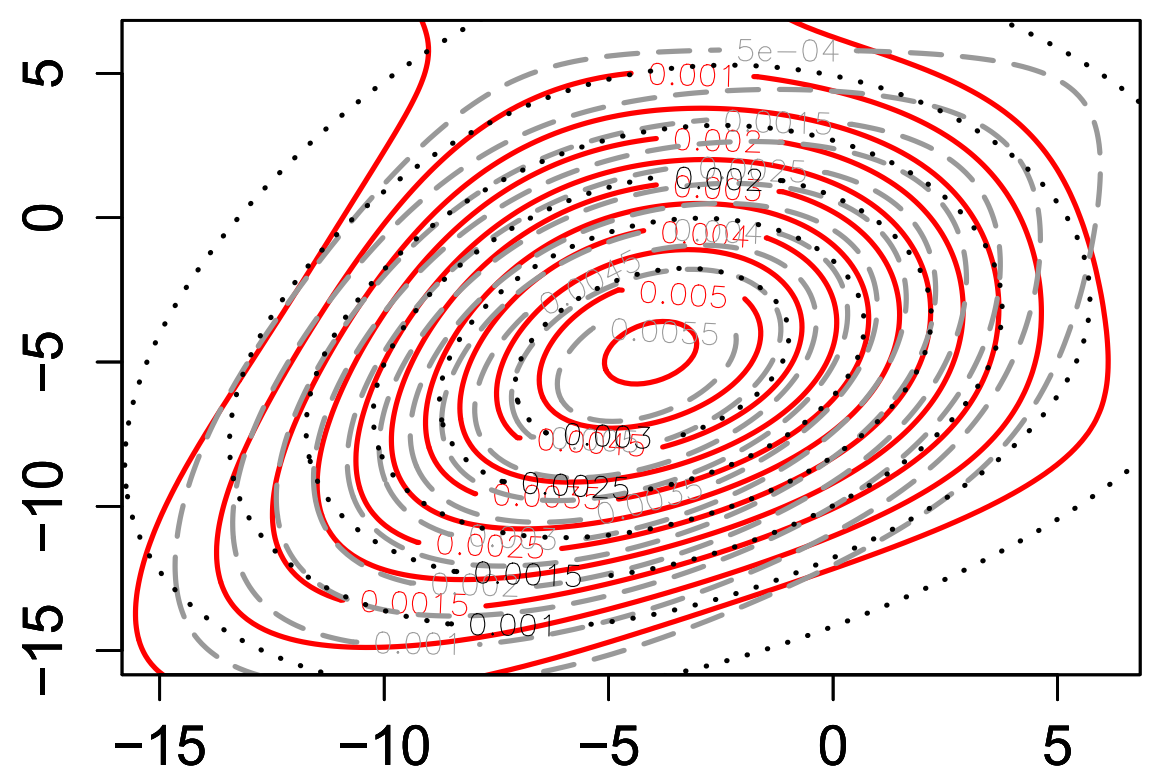

Notes: Contour plot. The solid red lines correspond to estimation via sample combination. The dashed grey lines correspond to estimation via inversion of the joint ECF of $X_{F H}, X_{M H}$ estimated on the bivariate sample. The dotted black lines correspond to direct non-parametric kernel estimation on the bivariate samples. $U=T=0.5$ was used. 
Figure 3.6: Estimated marginal PMFs - CC in volume

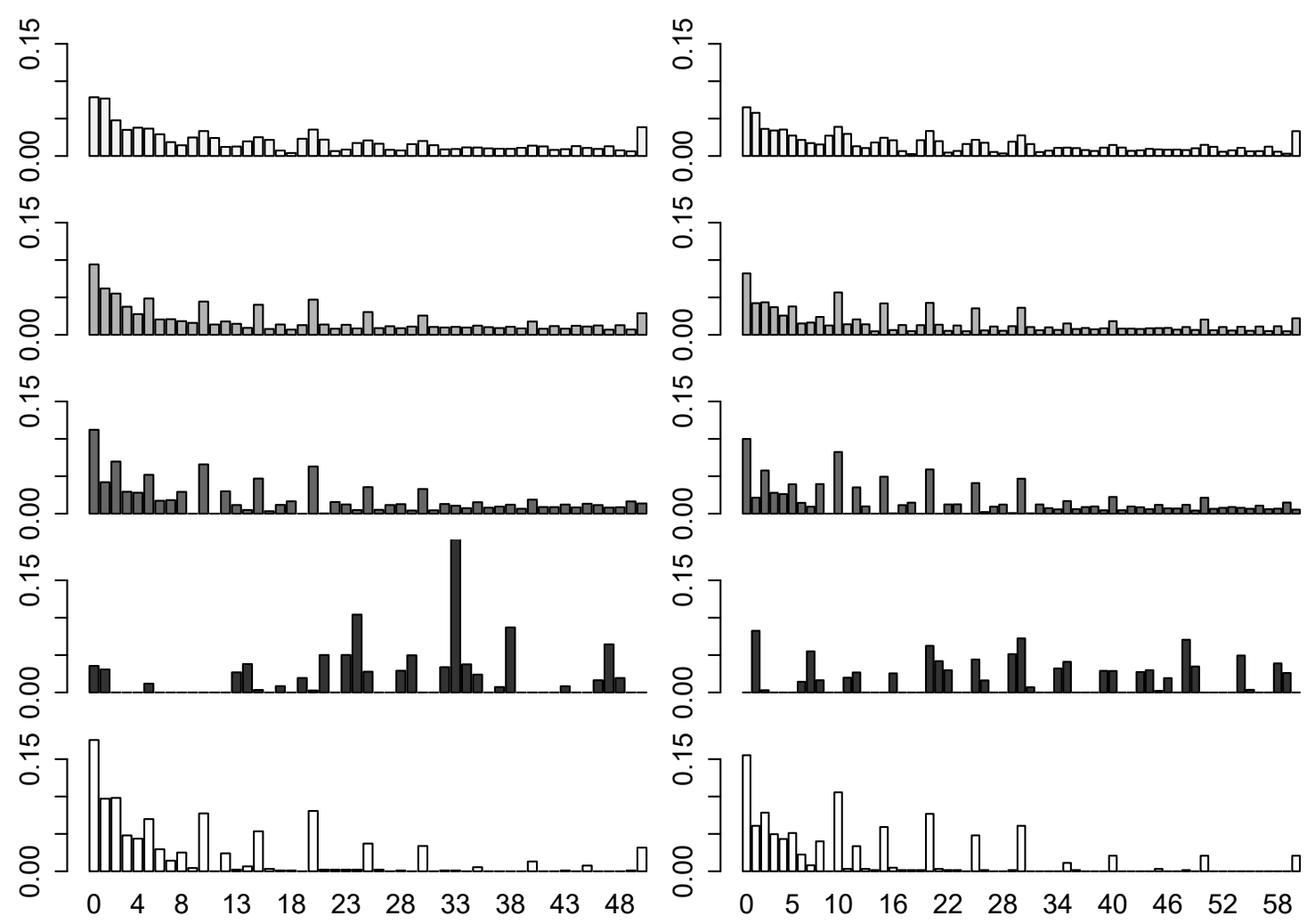

Notes: Marginal PMF estimates for female heads on the left, for male heads on the right. In various shades of grey, from top to bottom, PMF estimates based on sample combination with truncation values $(U=T)$ of $2,3,4$ and 5 . In white, empirical histograms obtained directly on the univariate samples. 
Figure 3.7: Estimated marginal PMFs - SVC in volume
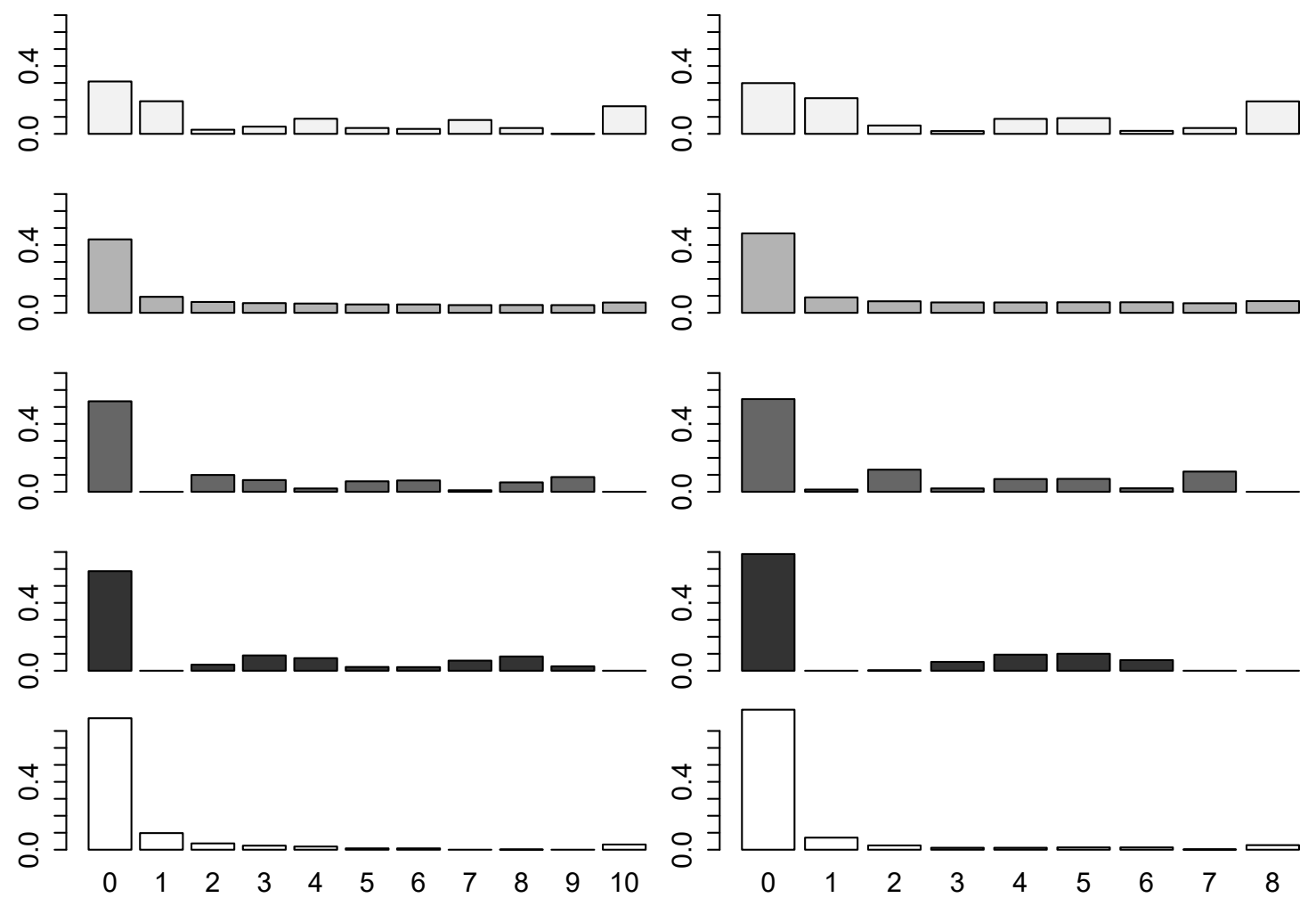

Notes: Marginal PMF estimates for female heads on the left, for male heads on the right. In various shades of grey, from top to bottom, PMF estimates based on sample combination with truncation values $(U=T)$ of $2,3,4$ and 5 . In white, empirical histograms obtained directly on the univariate samples. 
Figure 3.8: Estimated marginal PMFs - cash in volume

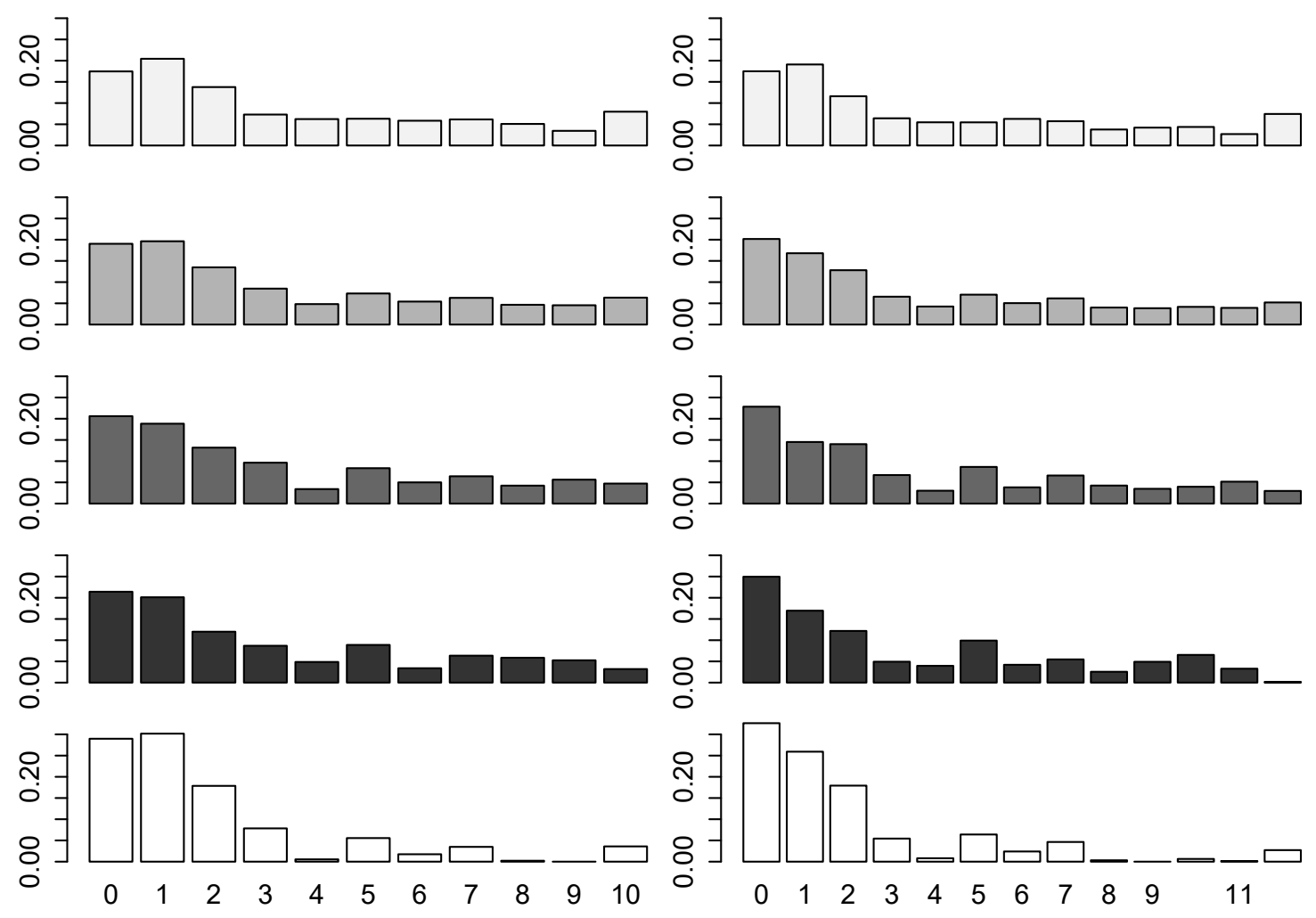

Notes: Marginal PMF estimates for female heads on the left, for male heads on the right. In various shades of grey, from top to bottom, PMF estimates based on sample combination with truncation values $(U=T)$ of $2,3,4$ and 5 . In white, empirical histograms obtained directly on the univariate samples. 
Figure 3.9: $\widehat{f}_{X_{F H}, X_{M H}}$ and $\widehat{f}_{X_{F H}} \widehat{f}_{X_{M H}}-\mathrm{CC}$ in volume
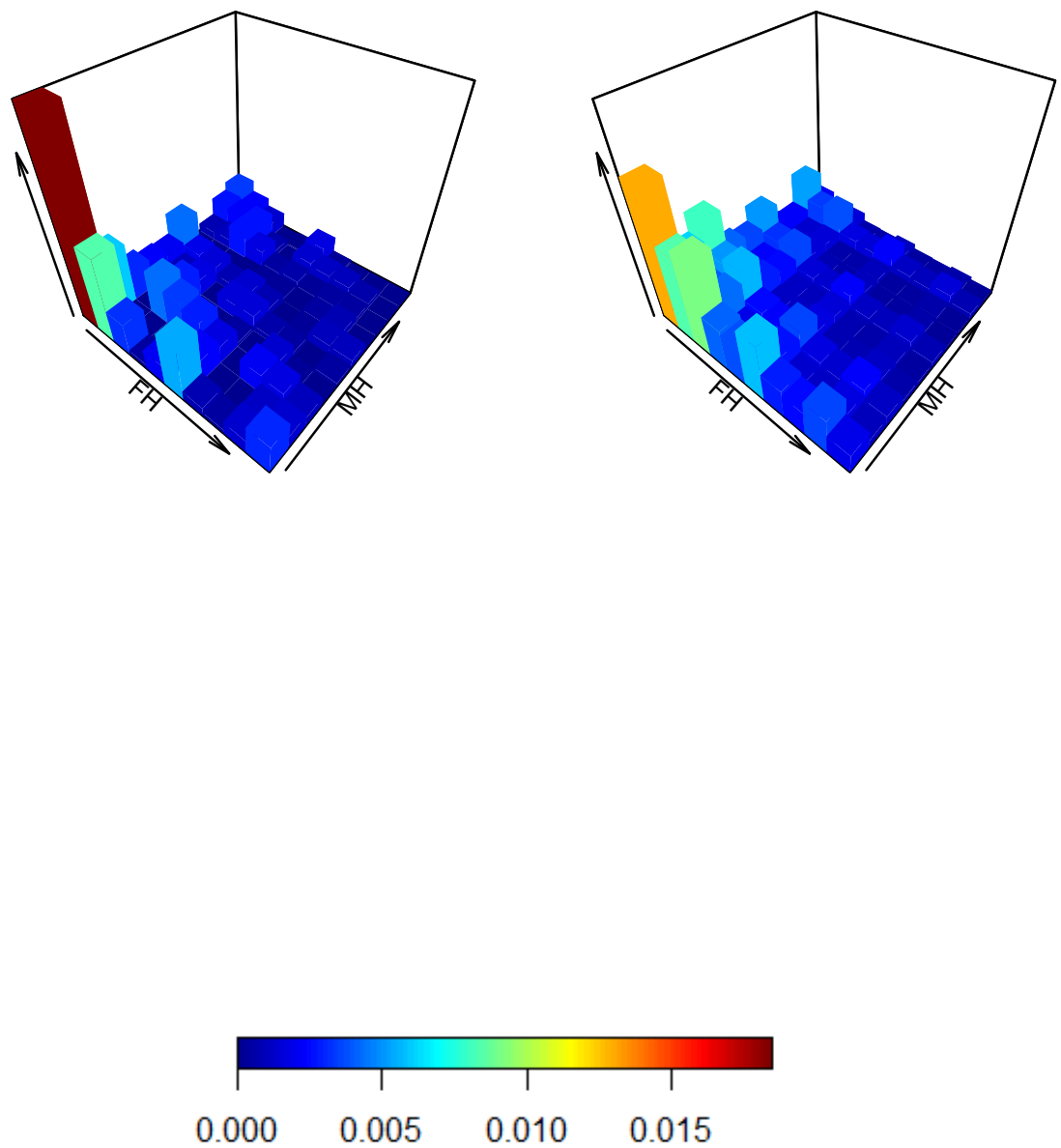

Notes: Joint PMF estimates are obtained by applying the proposed sample combination methodology under Model 1. Marginal PMF estimates are derived from the joint PMF estimates. Graphs illustrate distribution functions on the subset of their support $\{0,1, \ldots, 9\} \times\{0,1, \ldots, 9\}$. $T=U=4$ was used. 
Figure 3.10: $\widehat{f}_{X_{F H}, X_{M H}}$ and $\widehat{f}_{X_{F H}} \widehat{f}_{X_{M H}}-\mathrm{SVC}$ in volume
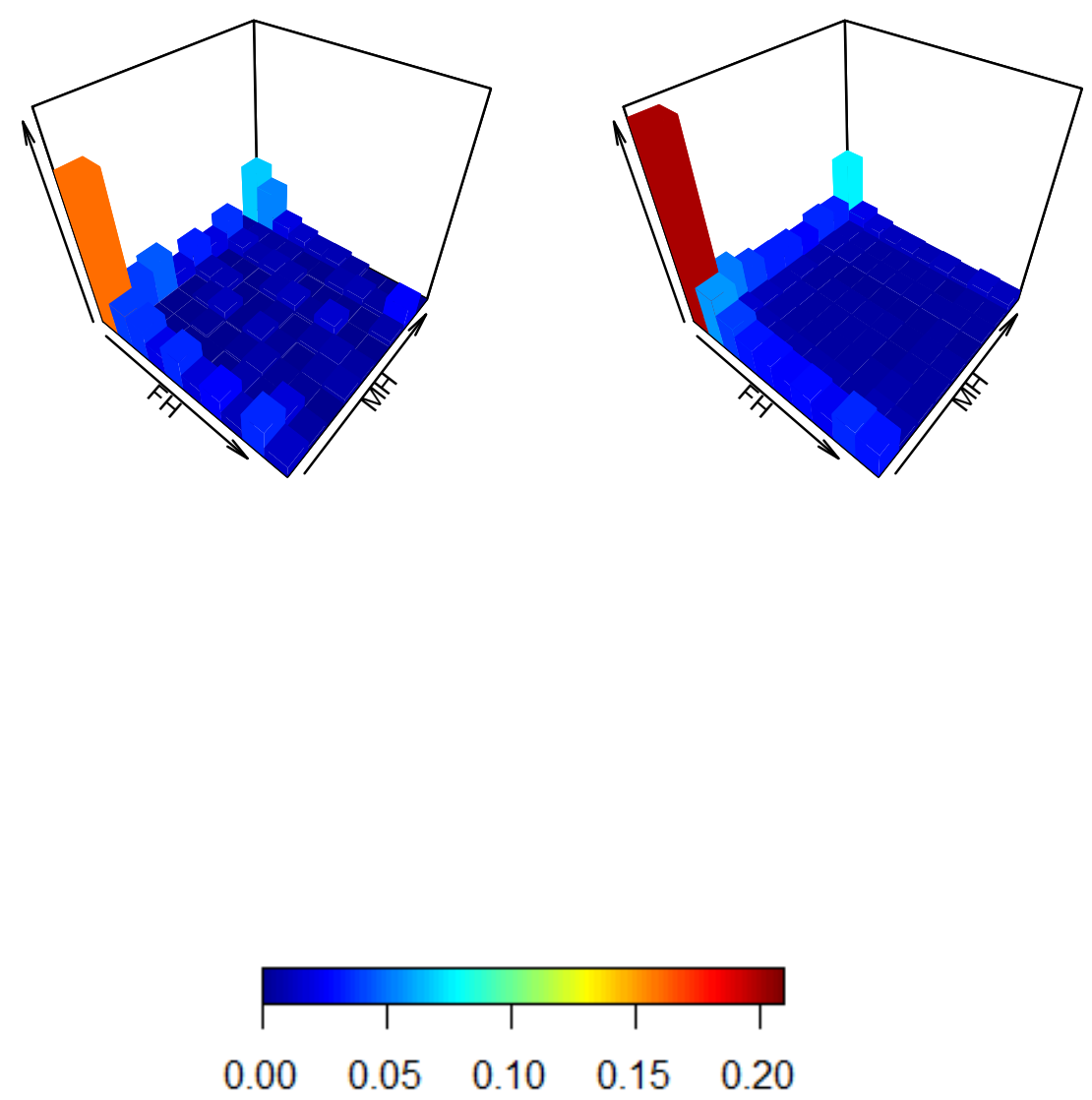

Notes: Joint PMF estimates are obtained by applying the proposed sample combination methodology under Model 1. Marginal PMF estimates are derived from the joint PMF estimates. Graphs illustrate distribution functions on the subset of their support $\{0,1, \ldots, 9\} \times\{0,1, \ldots, 9\}$. $T=U=3$ was used. 
Figure 3.11: $\widehat{f}_{X_{F H}, X_{M H}}$ and $\widehat{f}_{X_{F H}} \widehat{f}_{X_{M H}}$ - cash in volume
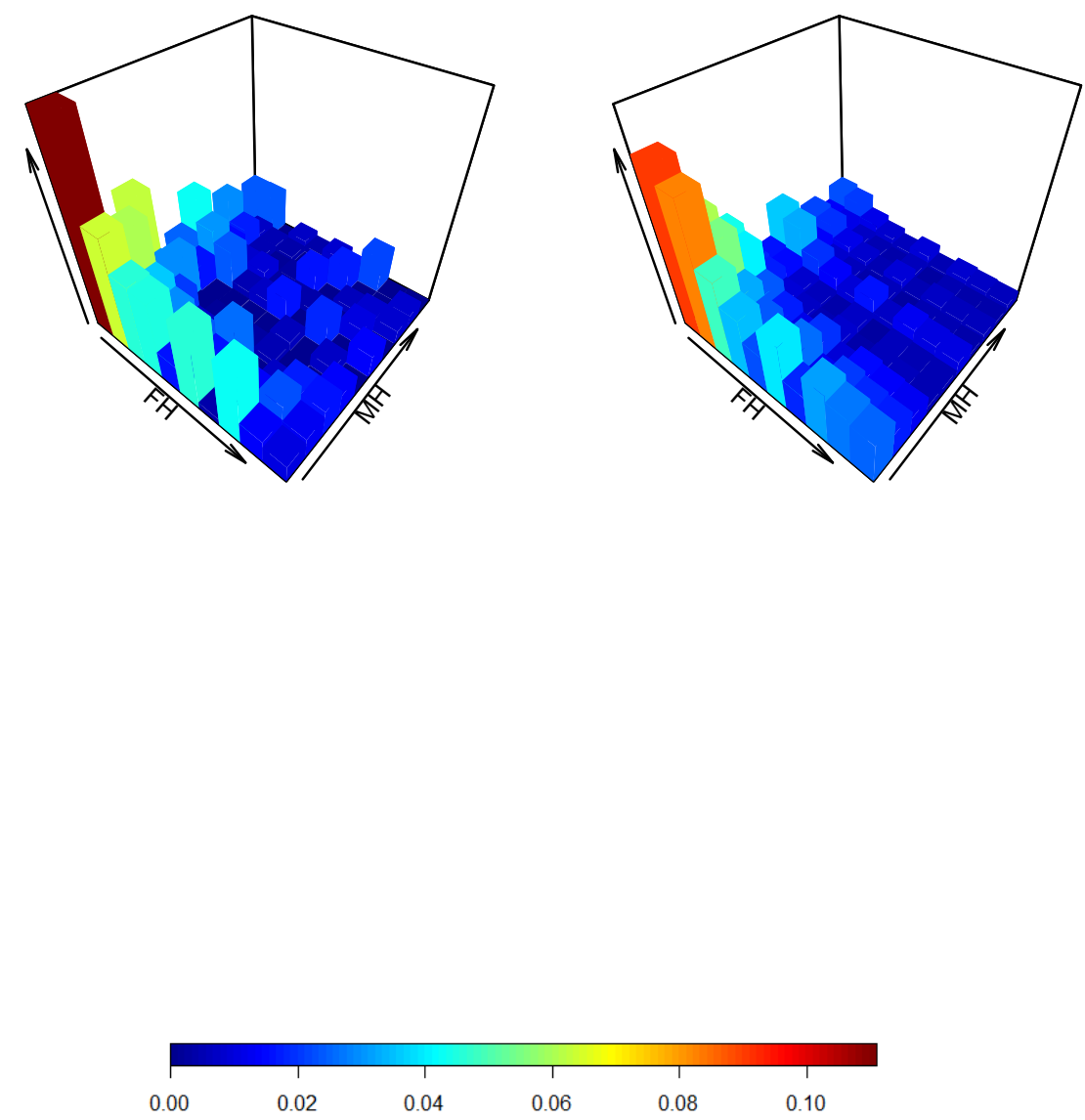

Notes: Joint PMF estimates are obtained by applying the proposed sample combination methodology under Model 1. Marginal PMF estimates are derived from the joint PMF estimates. Graphs illustrate distribution functions on the subset of their support $\{0,1, \ldots, 9\} \times\{0,1, \ldots, 9\}$. $T=U=5$ was used. 